Copyright

by

Simon De Rijck

2018 
The Dissertation Committee for Simon De Rijck

certifies that this is the approved version of the following dissertation:

\section{A Search for Large Extra Dimensions with MINOS and MINOS+}

Committee:

Karol Lang, Supervisor

Jack Ritchie

Peter Onyisi

Tim Andeen

Karl Gebhardt 


\title{
A Search for Large Extra Dimensions with MINOS and MINOS+
}

\author{
by
}

\section{Simon De Rijck}

\author{
DISSERTATION \\ Presented to the Faculty of the Graduate School of \\ The University of Texas at Austin \\ in Partial Fulfillment \\ of the Requirements \\ for the Degree of \\ DOCTOR OF PHILOSOPHY
}

THE UNIVERSITY OF TEXAS AT AUSTIN

May 2018 
Dedicated to my family. 


\section{Acknowledgments}

I would not have been able to complete this dissertation without the help and support of many people. First and foremost, I want to thank my advisor Karol Lang for his guidance and support during my years in graduate school. It was a pleasure working with him and his professional advice will greatly benefit me in the future.

In the UTKL group, I want to thank Thomas Carroll, Ramon Salazar, Dung Phan, Beatriz Tapia Oregui, John Cesar, Junting Huang, Adam Schreckenberger, Will Flanagan, Federico Nova, and Marek Proga for all the interesting and great moments we shared, both in Austin and at Fermilab.

In the MINOS and MINOS+ collaborations, I want to express my gratitude to Jacob Todd, Thomas Carroll, Rui Chen, Junting Huang, Adam Schreckenberger, Ashley Timmons, Navaneeth Poonthottathil, Adam Aurisano, Leigh Whitehead, Alex Sousa, Justin Evans, Jenny Thomas, Anna Holin, Greg Pawloski, Robert Plunkett, Art Kreymer, Robert Hatcher, Donatella Torretta, and many others for all the interesting conversations, help, and advice that greatly contributed to this dissertation. In addition, I want to thank Pedro Machado for the fruitful discussions we had about the LED model.

Finally, I want to thank my family and my girlfriend for their love, patience, and support during this journey. 


\title{
A Search for Large Extra Dimensions with MINOS and MINOS+
}

\author{
Publication No.
}

Simon De Rijck, Ph.D.

The University of Texas at Austin, 2018

Supervisor: Karol Lang

This dissertation presents the search for large extra dimensions using the rich and unique accelerator beam neutrino data collected by the MINOS and MINOS+ experiments from 2005 to 2012 and 2013 to 2016, respectively. Most data from neutrino oscillation experiments are consistent with the standard three-flavor paradigm. With increasing experimental precision, potential effects from physics beyond the Standard Model that could modify neutrino oscillations in subtle ways can be explored. One such scenario involves additional spatial dimensions. Following the Large Extra Dimensions (LED) model, sterile neutrinos arising as Kaluza-Klein states in an extra dimension with size $R$ can mix with the three active neutrinos in $3+1$ spacetime, thereby altering the neutrino oscillation probabilities. In addition, this model provides a natural explanation for small Dirac neutrino masses through mass suppression by the volume of the large extra dimensions. In this work, two analysis strategies are employed to search for large extra dimensions: the Far-over-Near 
analysis and the two-detector analysis. In the former, the ratio of the neutrino energy spectra measured in the MINOS Far and Near Detectors is used to constrain the LED model parameters, while in the latter the measured spectra are used simultaneously. MINOS reported the strongest constraint on $R$ from a neutrino oscillation experiment, constraining $R$ to be smaller than $0.45 \mu \mathrm{m}$ at $90 \%$ C.L. in the limit of a vanishing lightest neutrino mass, using the Farover-Near approach. Including MINOS+ data, which significantly increases the number of events at higher neutrino energies away from the three-flavor minimum where the model effects are larger, and using the two-detector technique, the size of extra dimensions is further constrained to be smaller than $0.30 \mu \mathrm{m}$ at $90 \%$ C.L. Stronger limits are obtained for non-vanishing lightest neutrino masses. 


\section{Table of Contents}

Acknowledgments $\quad$ v

Abstract vi vi

List of Tables $\quad$ xiii

List of Figures $\quad$ xiv

$\begin{array}{lll}\text { Chapter 1. Neutrino Oscillations } & 1\end{array}$

1.1 Neutrino Physics . . . . . . . . . . . . . . . . . . 2

1.2 Three-Flavor Neutrino Oscillations . . . . . . . . . . . . 5

1.2.1 Three-Flavor Formalism . . . . . . . . . . . . . 6

1.2.2 Two-Flavor Approximation . . . . . . . . . . . . 12

1.3 Beyond the Three-Flavor Paradigm . . . . . . . . . . . . . . . 14

Chapter 2. Large Extra Dimensions $\quad 20$

2.1 Large Extra Dimensions Model . . . . . . . . . . . . . . . . . 20

2.2 Status of Searches for Extra Dimensions . . . . . . . . . . . 23

2.2.1 Neutrino Oscillation Searches . . . . . . . . . . . 23

2.2 .2 Other Searches . . . . . . . . . . . . . . 24

2.3 Searching for Large Extra Dimensions with MINOS and MINOS+ 26

2.4 Interpreting the Large Extra Dimensions Model . . . . . . . . 39

2.4.1 A Back-of-the-Envelope Calculation of the Sensitivity to $R 42$

2.4.2 Mass Ordering for Disappearance Channels . . . . . . . 42

2.4.3 A Closer Look at the Kaluza-Klein Towers . . . . . . . . 44

2.4.4 Defining an Effective Mixing Angle . . . . . . . . . 49

viii 
Chapter 3. The MINOS and MINOS+ Experiments 51

3.1 The NuMI Neutrino Beam . . . . . . . . . . . . . . 52

3.2 The MINOS Detectors . . . . . . . . . . . . . 55

3.3 Neutrino Interactions in the MINOS Detectors . . . . . . . . 56

3.4 MINOS and MINOS+ Data . . . . . . . . . . . . 60

3.5 Detector Calibration and Performance . . . . . . . . . . . 64

3.5.1 Calibration Procedure . . . . . . . . . . . . . . 64

3.5.2 Long Term Performance of the Detectors . . . . . . . . 66

3.5.2.1 Detector Drift . . . . . . . . . . . 66

3.5.2.2 PMT Behavior . . . . . . . . . . . . 67

3.5.2.3 Detector Light Yield . . . . . . . . . . . . 68

3.5.2.4 Far Detector Timing Calibration . . . . . . . 70

3.5.3 Normalizing the Scintillator Response . . . . . . . . . 72

3.5.4 Relative Energy Scale . . . . . . . . . . . . . 73

3.6 Simulating the MINOS and MINOS+ Experiments . . . . . . 78

3.6 .1 NuMI Beam Simulation . . . . . . . . . . . . 78

3.6.1.1 SKZP Weights . . . . . . . . . . . 78

3.6.1.2 PPFX Weights . . . . . . . . . . . . . 79

3.6.2 MINOS Detector Simulation _. . . . . . . . . . 80

3.7 Event Reconstruction . . . . . . . . . . . . . . . . 80

3.7.1 Intensity Effect . . . . . . . . . . . . . . . . . . 84

Chapter 4. Event Selection $\quad 85$

4.1 Preselection . . . . . . . . . . . . . . . 86

4.2 Neutral Current Event Selection . . . . . . . . . . . . . . 87

4.2 .1 Acceptance Selection . . . . . . . . . . . . . . . . . . 89

4.2 .2 Topology Requirements . . . . . . . . . . . . . . . . 92

4.3 Charged Current Event Selection _. . . . . . . . . . . 92

4.3.1 Acceptance Selection . . . . . . . . . . . . . . . . . 95

4.3.2 Separating Charged and Neutral Current Events . . . . 95

4.4 Event Selection Performance . . . . . . . . . . . . . . 97

4.5 Selected Samples . . . . . . . . . . . . . . . . . . . 99 
Chapter 5. A Far-Over-Near Analysis 104

5.1 Analysis Method . . . . . . . . . . . . . . . . 105

5.1 .1 Test Statistic . . . . . . . . . . . . . . . . 106

5.1 .2 Ghost Fitter . . . . . . . . . . . . . . . . . . . 107

5.1.3 Corrections to the Monte Carlo Simulation . . . . . . 110

5.1.4 Dynamic Scaling of the Covariance Matrix . . . . . . . . 111

5.2 Systematic Uncertainties . . . . . . . . . . . . . . . . 118

5.2.1 Constructing Uncertainty Bands . . . . . . . . . . . . 118

5.2 .2 Constructing Covariance Matrices . . . . . . . . . . . 119

5.2 .3 Total Systematic Uncertainty . . . . . . . . . . . . . 120

5.2 .4 Acceptance Uncertainty . . . . . . . . . . . . . . . . 120

5.2 .5 Normalization Uncertainty . . . . . . . . . . . . . . 124

5.2.6 Neutral Current Selection Uncertainty . . . . . . . . . 126

5.2 .7 Energy Scale Uncertainties . . . . . . . . . . . . 126

5.2 .8 Neutrino Cross Section Uncertainty . . . . . . . . . 133

5.2 .9 Background Uncertainty . . . . . . . . . . . . . . . 133

5.2.10 Hadron Production Uncertainty . . . . . . . . . . . . 135

5.2 .11 Beam Optics Uncertainty . . . . . . . . . . . . . 135

5.3 Sensitivity and Data $\chi^{2}$ Surfaces . . . . . . . . . . . . . 139

5.4 Feldman-Cousins Data Limit . . . . . . . . . . . . . . . 140

5.4.1 Interpreting the Sensitivity and Data $\chi^{2}$ Surfaces . . . . 146

5.4 .2 Feldman-Cousins Study . . . . . . . . . . . . . . . 147

5.4.3 The Asimov and Median Sensitivity . . . . . . . . . 154

5.5 Including MINOS+ Data . . . . . . . . . . . . 156

Chapter 6. A Two-Detector Analysis 164

6.1 Analysis Method . . . . . . . . . . . . . . . . 165

6.1.1 Correcting the FLUGG Neutrino Flux Prediction . . . . 165

6.1.2 Test Statistic and Ghost Fitter . . . . . . . . . . . . 166

6.2 Systematic Uncertainties . . . . . . . . . . . . . . . . . 169

6.2 .1 Total Systematic Uncertainty . . . . . . . . . . . . 172

6.2 .2 Acceptance Uncertainty . . . . . . . . . . . . . . . . 172 
6.2 .3 Normalization Uncertainty . . . . . . . . . . . . . 172

6.2 .4 Neutral Current Uncertainty . . . . . . . . . . . . 175

6.2 .5 Energy Scale Uncertainties _. . . . . . . . . . . 178

6.2.6 Neutrino Cross Section Uncertainty . . . . . . . . . 178

6.2 .7 Background Uncertainty . . . . . . . . . . . . . . . . 181

6.2 .8 Hadron Production Uncertainty . . . . . . . . . . . . . 181

6.2 .9 Run Period Weight Uncertainty . . . . . . . . . . . . . 181

6.2 .10 Beam Optics Uncertainty . . . . . . . . . . . . . . . 184

6.3 Sensitivity and Data $\chi^{2}$ Surfaces . . . . . . . . . . . . . 187

6.4 Feldman-Cousins Data Limit . . . . . . . . . . . . . . . 188

6.4.1 Interpreting the Sensitivity and Data $\chi^{2}$ Surfaces . . . . 188

6.4.2 Feldman-Cousins Study . . . . . . . . . . . . . . . . 194

6.4.3 Constructing a Sensitivity Band . . . . . . . . . . 198

$\begin{array}{lll}\text { Chapter 7. } & \text { Results and Outlook } & 206\end{array}$

$\begin{array}{ll}\text { Appendices } & 208\end{array}$

Appendix A. Validating the Fluctuation Procedure 209

A.1 Reconstructing the Covariance Matrix from Fluctuated Simulated Data Spectra . . . . . . . . . . . . . . . . . 210

A.2 A Few Examples. . . . . . . . . . . . . . . . . 210

Appendix B. A Study of the Asimov Sensitivity Coverage 222

B.1 Introduction . . . . . . . . . . . . . . . . . 223

B.2 An ND CC Sample Study _. . . . . . . . . . . . 228

B.2.1 Observations . . . . . . . . . . . . . . . 228

B.2.2 Discussion . . . . . . . . . . . . . . . . . 231

B.3 A Fully Correlated 5\% Normalization Uncertainty Study . . . 240

B.3.1 Mimicking Sterile Neutrino Oscillation Signals for High $\Delta m_{41}^{2}$ Values . . . . . . . . . . . . . . . 240

B.3.2 Turning Off Correlations in the Fluctuation Process . . 242

B.3.3 Turning Off Correlations in the $\chi^{2}$ Calculation . . . . . 244

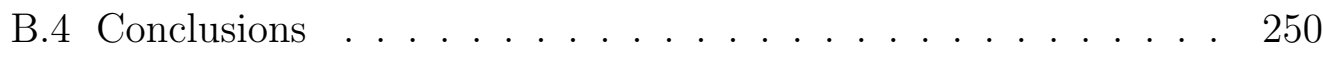


Bibliography

251

Vita

270

xii 


\section{List of Tables}

1.1 Global fit result of the standard three-flavor oscillation model taken from Ref. [31]. Note that $\delta m^{2} \equiv \Delta m_{21}^{2}$ and $\Delta m^{2} \equiv$ $m_{3}^{2}-\left(m_{1}^{2}-m_{2}^{2}\right) / 2$, with $+\Delta m^{2}$ for normal mass ordering (NO) and $-\Delta m^{2}$ for inverted mass ordering (IO). . . . . . . . .

5.1 The values and constraints on the neutrino oscillation parameters used in the LED fitting procedure that minimizes Formula (5.1). . . . . . . . . . . . . . .

5.2 The number of events and Far-over-Near ratio in each energy bin for the toy model spectra used in Fig. 5.3 to illustrate static versus dynamic scaling for the statistical variance. . . . . . . .

5.3 Systematic uncertainties in the absolute energy scale derived from CalDet data and cosmic muon data in the ND and FD.

5.4 Systematic uncertainties in the relative energy scale determination at the ND and FD. . . . . . . . . . . . .

5.5 The three-flavor oscillation parameter values used to generate the three-flavor simulated data. . . . . . . . . . . . .

5.6 The $\Delta \chi^{2}=\chi^{2}-\chi_{\min }^{2}$ values for the $68 \%, 90 \%, 95 \%$, and $95 \%$ confidence levels (C.L.) for 1 to 4 degrees of freedom $(\alpha)$. The $\mathrm{P}$-value is given by 1-C.L.(\%). . . . . . . . . . . . 


\section{List of Figures}

1.1 Measurements of the hadron production cross section around the $\mathrm{Z}$ boson resonance. The data included in this figure consist of 17 million $\mathrm{Z}$ decays accumulated by the ALEPH, DELPHI, L3 and OPAL experiments at LEP. The curves represent the predicted cross section for two, three, and four neutrino species with SM couplings and negligible mass. This figure is taken from Ref. [9]. . . . . . . . . . . . . . . . . . . .

1.2 The three-flavor neutrino oscillation paradigm. (Left) Illustration of the two possible neutrino mass orderings, normal and inverted ordering. The color coding indicates the fraction $\left|U_{\alpha i}\right|^{2}$ of flavor $v_{\alpha}(\alpha=\mathrm{e}, \mu, \tau)$ contained in mass eigenstate $i$. This figure is a modified version of a figure taken from Ref. [33]. (Right) The muon neutrino disappearance (blue line), tau neutrino appearance (red line), and electron neutrino appearance (green line) probabilities as a function of $L / E$, with $L$ the distance traveled by the neutrino and $E$ the neutrino energy, for the three-flavor case. The oscillation parameters are fixed for all cases [32]: $\Delta m_{32}^{2}=2.37 \times 10^{-3} \mathrm{eV}^{2}, \theta_{23}=0.695, \theta_{13}=0.149$, $\delta_{\mathrm{CP}}=0, \Delta m_{21}^{2}=7.54 \times 10^{-5} \mathrm{eV}^{2}$, and $\theta_{12}=0.588$. The $L / E$ coverage of the MINOS Near and Far Detectors are represented by the gray bands, which contain $90 \%$ of the MINOS and MINOS+ events between 0 and $40 \mathrm{GeV}$. The MINOS energy resolution is taken into account. . . . . . . . . . . . .

1.3 A combined three-flavor fit to MINOS muon neutrino disappearance and electron neutrino appearance data and atmospheric neutrino data. (Left) The $68 \%$ and $90 \%$ C.L. limits in the $\left(\sin ^{2} \theta_{23}, \Delta m_{32}^{2}\right)$ plane calculated for normal ordering (top) and inverted ordering (bottom). (Right) The log-likelihood profiles for each mass ordering for $\Delta m_{32}^{2}$ (top) and $\sin ^{2} \theta_{23}$ (bottom). This figure is taken from Ref. [30]. . . . . . . . . . 
1.4 The short-baseline anomalies in LSND and MiniBooNE. (Left) A two-flavor fit in the $\left(\sin ^{2}(2 \theta), \Delta m^{2}\right)$ plane for the entire LSND data sample, with $20<E_{\mathrm{e}}<200 \mathrm{MeV}$. The fit includes primary $\bar{v}_{\mu} \rightarrow \bar{v}_{\mathrm{e}}$ oscillations and secondary $v_{\mu} \rightarrow v_{\mathrm{e}}$ oscillations, as well as all known neutrino backgrounds. The allowed $90 \%$ C.L. and $99 \%$ C.L. regions are shown in blue and yellow, respectively. Other curves are 90\% C.L. limits from the Bugey reactor experiment [40], the CCFR experiment at Fermilab [41], the NOMAD experiment at CERN [42], and the KARMEN experiment at ISIS [43]. This figure is taken from Ref. [34]. (Right) The MiniBooNE allowed regions in the $\left(\sin ^{2}(2 \theta), \Delta m^{2}\right)$ plane in a combined neutrino and antineutrino mode for events with $200<E_{\gamma}^{\mathrm{QE}}<3000 \mathrm{MeV}$ within a two-neutrino $v_{\mu} \rightarrow v_{\mathrm{e}}$ and $\bar{v}_{\mu} \rightarrow \bar{v}_{\mathrm{e}}$ oscillation model. Also shown is the $\bar{v}_{\mu} \rightarrow \bar{v}_{\mathrm{e}}$ limit from the KARMEN experiment [44]. The shaded areas show the 90\% and $99 \%$ C.L. LSND $\bar{v}_{\mu} \rightarrow \bar{v}_{\mathrm{e}}$ allowed regions. The black star shows the best fit point. This figure is taken from Ref. [35]. The legend was modified for clarity. . . . . . . . . . . . .

1.5 The effect of $\Delta m_{41}^{2}$ (top row) and $\theta_{24}$ (bottom row) on the muon disappearance (left column) and sterile appearance (right column) probabilities as a function of $L / E$ in the sterile $3+1$ model. Unless indicated otherwise, the parameter values are given by: $\Delta m_{21}^{2}=7.54 \times 10^{-5} \mathrm{eV}^{2}, \Delta m_{21}^{2}=2.37 \times 10^{-3} \mathrm{eV}^{2}$, $\Delta m_{41}^{2}=0.5 \mathrm{eV}^{2}, \theta_{12}=0.554, \theta_{13}=0.149, \theta_{23}=0.695, \theta_{14}=0$, $\theta_{24}=0.2, \theta_{34}=0.5, \delta_{13}=0, \delta_{14}=0$, and $\delta_{24}=0$. These figures are taken from Ref. [45]. . . . . . . . . . . . . . 
1.6 Constraining the $3+1$ sterile neutrino model with MINOS. (Left) The Feldman-Cousins 90\% C.L. data coverage for MINOS using $v_{\mu}$ disappearance and searching for a deficit in neutral current events. These contours are compared to other experiments. Note the axis is $\sin ^{2}\left(\theta_{24}\right)$. All experiments except Super-K and MINOS reported results in terms of $\sin ^{2}\left(2 \theta_{24}\right)$, so their data coverages were made symmetric when converting to $\sin ^{2}\left(\theta_{24}\right)$. Also shown are the $90 \%$ C.L. allowed regions of two global fits. The Kopp et al. 2013 fit included $v_{\mathrm{e}}$ appearance experiments, and the Gariazzo et al. 2016 fit included all short-baseline experiments with the exception of MiniBooNE data below $475 \mathrm{MeV}$. To compare these global fits to disappearance data, $\sin ^{2} 2 \theta_{\mu \mathrm{e}}$ is converted to $\sin ^{2} \theta_{24}$ by assuming $\theta_{14}=0.15$, the best fit value from the Kopp et al. global fit to appearance data. This figure is taken from Ref. [47]. (Right) The MINOS and Daya Bay/Bugey-3 combined $90 \% \mathrm{CL}_{\mathrm{S}}$ data limit on $\sin ^{2} 2 \theta_{\mu \mathrm{e}} \mathrm{com}-$ pared to the LSND and MiniBooNE 90\% C.L. allowed regions. Regions of parameter space to the right of the red contour are excluded. The regions excluded at 90\% C.L. by KARMEN2 and NOMAD are also shown. The excursion to small mixing in the exclusion contour at about $\Delta m_{41}^{2} \sim 5 \times 10^{-3} \mathrm{eV}^{2}$ is due to the degenerate island in the MINOS exclusion limit. This figure is taken from Ref. [48]. . . . . . . . . . . . . . . .

2.1 Searches for extra dimensions with neutrino oscillations. (Top left) The MINOS sensitivity to the LED model in the $\left(R, m_{0}\right)$ plane as calculated in Ref. [64]. (Bottom left) The sensitivity to the LED model obtained from atmospheric neutrino data collected by the IceCube experiment (red and blue dashed lines) and presented in Ref. [65]. The other confidence regions in this figure are estimated sensitivities. (Top right) The relation between the LED model and the $3+3 n$ sterile neutrino model for $n=3$, where $n$ is the number of KK modes considered in each tower, in the muon antineutrino disappearance channel calculated in Ref. [65]. (Bottom right) The sensitivity to the LED + model for MINOS (solid gray line), reactor short baseline neutrino oscillation experiments (gray dashed line), DUNE (red dashed line), and SBN (blue line), calculated in Ref. [68] (unit conversion: $\left.1 \mu \mathrm{m}=5 \mathrm{eV}^{-1}\right)$............... 
2.2 World's search for extra dimensions. The plot is provided by Machado, Nunokawa and Zukanovich Funchal. $M_{D}, \delta$, and $R$ are the fundamental Planck scale in the bulk, the number of extra dimensions, and the extra dimension size, respectively, where the first two are introduced in Formula (2.1) as $\bar{M}_{\mathrm{Pl}}$ and $d$. The left green dashed line $\left(M_{D}>1700 \mathrm{TeV}\right.$ for $\left.\delta=2\right)$ is the limit from requiring that neutron stars are not excessively heated by KK graviton decays into photons [69]. The right green dashed line $\left(M_{D}>100 \mathrm{TeV}\right.$ for $\left.\delta=2\right)$ is the cosmological constraint based on the restriction on the amount of relic gravitons in the universe [70]. The solid blue line $\left(M_{D}>27 \mathrm{TeV}\right.$ for $\delta=2$ ) is the limit from supernova SN1987A requiring that the graviton luminosity agrees with certain stellar models [71]. The MINOS limit shown in blue is based on the simulations done by the authors of this plot. The purple dashed line $(R<37 \mu \mathrm{m}$ at 95\% CL for $n=2$ ) is the limit from torsion balance experiments [72]. The right-most red circle $\left(M_{D}>1.60 \mathrm{TeV}\right.$ for $\left.\delta=2\right)$ is the limit from a combination of the LEP results on graviton emission $[73,74] . \ldots \ldots \ldots \ldots$

2.3 The muon neutrino survival probability $P\left(v_{\mu} \rightarrow v_{\mu}\right)$ at the MINOS FD as a function of the true neutrino energy for $m_{0}=0 \mathrm{eV}$ and $R=0.5 \mu \mathrm{m}$ (red line) or $1 \mu \mathrm{m}$ (blue line), and for threeflavor oscillation (black line). . . . . . . . . . . . .

2.4 The muon neutrino survival probability $P\left(v_{\mu} \rightarrow v_{\mu}\right)$ at the MINOS ND (top left) and FD (top right) and the sterile neutrino appearance probability $P\left(v_{\mu} \rightarrow v_{\mathrm{s}}\right)$ at the ND (bottom left) and FD (bottom right) as a function of the neutrino energy for the three-flavor case (red lines) and for $R=1.00 \mu \mathrm{m}$ and $m_{0}=0.000 \mathrm{eV}$ (blue lines). The effects of the MINOS CC energy resolution are also shown for both baselines. . . . . . . .

2.5 The muon neutrino survival probability $P\left(v_{\mu} \rightarrow v_{\mu}\right)$ at the MINOS ND (top left) and FD (top right) and the sterile neutrino appearance probability $P\left(v_{\mu} \rightarrow v_{\mathrm{s}}\right)$ at the ND (bottom left) and FD (bottom right) as a function of the neutrino energy for the three-flavor case (red lines) and for $R=0.50 \mu \mathrm{m}$ and $m_{0}=0.000 \mathrm{eV}$ (blue lines). The effects of the MINOS CC energy resolution are also shown for both baselines. . . . . . . .

2.6 The muon neutrino survival probability $P\left(v_{\mu} \rightarrow v_{\mu}\right)$ at the MINOS ND (top left) and FD (top right) and the sterile neutrino appearance probability $P\left(v_{\mu} \rightarrow v_{\mathrm{s}}\right)$ at the ND (bottom left) and FD (bottom right) as a function of the neutrino energy for the three-flavor case (red lines) and for $R=0.50 \mu \mathrm{m}$ and $m_{0}=0.100 \mathrm{eV}$ (blue lines). The effects of the MINOS CC energy resolution are also shown for both baselines. . . . . . . 
2.7 The effect of the matter effect (left), the mass ordering (middle), and the number of KK modes (right) on the muon disappearance (top) and sterile appearance (bottom) oscillation probabilities in the MINOS detectors, taking into account energy resolution effects. Parameters not shown in the figures are fixed to their three-flavor values, as shown in Fig. 2.3. . . . . .

2.8 Effect of $\Delta m_{32}^{2}$ (top) and $\theta_{23}$ (bottom) on the muon disappearance probability (left) and the sterile appearance probability (right) in the MINOS detectors, taking energy resolution effects into account. For the top plots, $\theta_{23}$ is fixed to 0.695, while for the bottom plots $\Delta m_{32}^{2}$ is fixed to $2.37 \times 10^{-3} \mathrm{eV}^{2}$. The other oscillation parameters are fixed for all cases: $R=0.40 \mu \mathrm{m}$, $m_{0}=0.100 \mathrm{eV}, \theta_{13}=0.149, \delta_{\mathrm{CP}}=0, \Delta m_{21}^{2}=7.54 \times 10^{-5} \mathrm{eV}^{2}$, and $\theta_{12}=0.588$. The $L / E$ coverage of the ND and FD are represented by the grey bands, which contain $90 \%$ of the MINOS and MINOS+ events between 0 and $40 \mathrm{GeV}$. . . . . . . . . .

2.9 Effect of $R$ (top) and $m_{0}$ (bottom) on the muon disappearance probability (left) and the sterile appearance probability (right) in the MINOS detectors, taking energy resolution effects into account. For the top plots, $m_{0}$ is fixed to $0.100 \mathrm{eV}$, while for the bottom plots $R$ is fixed to $0.40 \mu \mathrm{m}$. The other oscillation parameters are fixed for all cases: $\Delta m_{32}^{2}=2.37 \times 10^{-3} \mathrm{eV}^{2}$, $\theta_{23}=0.695, \theta_{13}=0.149, \delta_{\mathrm{CP}}=0, \Delta m_{21}^{2}=7.54 \times 10^{-5} \mathrm{eV}^{2}$, and $\theta_{12}=0.588$. The $L / E$ coverage of the ND and FD are represented by the grey bands, which contain $90 \%$ of the MINOS and MINOS+ events between 0 and $40 \mathrm{GeV}$. . . . . . . . .

2.10 Effect of $\theta_{13}$ (top) and $\delta_{\mathrm{CP}}$ (bottom) on the muon disappearance probability (left) and the sterile appearance probability (right) in the MINOS detectors, taking energy resolution effects into account. For the top plots, $\delta_{\mathrm{CP}}$ is fixed to 0 , while for the bottom plots $\theta_{13}$ is fixed to 0.149 . The other oscillation parameters are fixed for all cases: $R=0.40 \mu \mathrm{m}, m_{0}=0.100 \mathrm{eV}, \Delta m_{32}^{2}=2.37 \times$ $10^{-3} \mathrm{eV}^{2}, \theta_{23}=0.695, \Delta m_{21}^{2}=7.54 \times 10^{-5} \mathrm{eV}^{2}$, and $\theta_{12}=0.588$. The $L / E$ coverage of the ND and FD are represented by the grey bands, which contain $90 \%$ of the MINOS and MINOS+ events between 0 and $40 \mathrm{GeV}$. . . . . . . . . . . . . . 
2.11 Effect of $\Delta m_{21}^{2}$ (top) and $\theta_{12}$ (bottom) on the muon disappearance probability (left) and the sterile appearance probability (right) in the MINOS detectors, taking energy resolution effects into account. For the top plots, $\theta_{12}$ is fixed to 0.588 , while for the bottom plots $\Delta m_{21}^{2}$ is fixed to $7.54 \times 10^{-5} \mathrm{eV}^{2}$. The other oscillation parameters are fixed for all cases: $R=$ $0.40 \mu \mathrm{m}, m_{0}=0.100 \mathrm{eV}, \Delta m_{32}^{2}=2.37 \times 10^{-3} \mathrm{eV}^{2}, \theta_{23}=0.695$, $\theta_{13}=0.149$, and $\delta_{\mathrm{CP}}=0$. The $L / E$ coverage of the ND and FD are represented by the grey bands, which contain $90 \%$ of the MINOS and MINOS+ events between 0 and $40 \mathrm{GeV}$. . . .

2.12 The values of $\xi_{1}=\sqrt{2} m_{1} R$ (left), $\xi_{2}=\sqrt{2} m_{2} R$ (middle), and $\xi_{3}=\sqrt{2} m_{3} R$ (right) in the $\left(R, m_{0}\right)$ plane assuming normal mass ordering. . . . . . . . . . . . . . .

2.13 The difference between the first KK mass and the active neutrino mass (top) and the difference between the square of the first KK mass and the square of the active neutrino mass (bottom $)$ in each tower in the $\left(R, m_{0}\right)$ plane for the small $\xi_{i}$ approximation and assuming normal mass ordering. . . . . . . . . .

2.14 Effect of the LED parameter $R$ on the neutrino masses and oscillation probabilities. (Top) For fixed $m_{0} \equiv m_{1}=0.100 \mathrm{eV}$ (normal mass ordering), the effect of increasing $R$ on the active neutrino masses $m_{1}, m_{2}$, and $m_{3}$ and on the sterile neutrino masses in the KK towers $m_{\mathrm{KK}, i}^{(k)}$ (with $i=1,2,3$ and $\left.k=1, \ldots,+\infty\right)$ is demonstrated. The absolute masses of the active neutrinos are determined by $m_{0}$ and the known mass squared splittings $\Delta m_{32}^{2}$ and $\Delta m_{21}^{2}$, while the absolute masses of the sterile neutrinos are approximately proportional to the inverse of $R$. (Bottom) Comparison of the three-flavor (red) $v_{\mu}$ disappearance probability with the three LED (blue) cases from (top). With respect to the three-flavor case, the LED probabilities show a displacement of the oscillation minimum, a reduction of the integrated survival probability, and modulations due to fast oscillations with the sterile neutrinos in the KK towers. The effect of the CC energy resolution on the oscillation probability in the MINOS FD is shown as well. . . . . . . . . . . . . . 
2.15 Effect of the LED parameter $m_{0}$ on the neutrino masses and oscillation probabilities. (Top) For fixed $R=0.2 \mu \mathrm{m}$ (normal mass ordering), the effect of increasing $m_{0}$ on the active neutrino masses $m_{1}, m_{2}$, and $m_{3}$ and on the sterile neutrino masses in the KK towers $m_{\mathrm{KK}, i}^{(k)}$ (with $i=1,2,3$ and $\left.k=1, \ldots,+\infty\right)$ is demonstrated. The absolute masses of the active neutrinos are determined by $m_{0}$ and the known mass squared splittings $\Delta m_{32}^{2}$ and $\Delta m_{21}^{2}$, while the absolute masses of the sterile neutrinos are approximately proportional to the inverse of $R$. (Bottom) Comparison of the three-flavor (red) $v_{\mu}$ disappearance probability with the three LED (blue) cases from (top). With respect to the three-flavor case, the LED probabilities show a displacement of the oscillation minimum, a reduction of the integrated survival probability, and modulations due to fast oscillations with the sterile neutrinos in the KK towers. The effect of the CC energy resolution on the oscillation probability in the MINOS FD is shown as well. . . . . . . . . . . . . . .

2.16 The effective mixing angle as $\sin ^{2} \theta_{\text {eff }}^{\mu \mu}$ in the $\left(R, m_{0}\right)$ plane assuming normal mass ordering. . . . . . . . . . . . .

3.1 A schematic of the NuMI neutrino beam, showing the graphite target, the magnetic horns, the decay pipe, the hadron monitor, and the muon shielding. This figure is taken from Ref. [82]. . .

3.2 Creating a muon neutrino or antineutrino beam with NuMI. (Top) When positive pions and kaons are focused into the decay pipe by the magnetic horns, their decays lead to a neutrino beam dominated by muon neutrinos. For MINOS, the target was inserted $50.4 \mathrm{~cm}$ into the first horn to maximize the neutrino flux in the $1-3 \mathrm{GeV}$ range. For MINOS+, the target was moved outside the first horn and the second horn was moved further away from the first horn, $19.2 \mathrm{~m}$ from the upstream end of the first horn, leading to a neutrino flux peaking at $6-7 \mathrm{GeV}$. (Bottom) Focusing negative pions and kaons by reversing the horn current leads to a neutrino beam dominated by muon antineutrinos. This configuration was only adopted in the MINOS era. . . . . . . . . . . . . . . .

3.3 The MINOS and MINOS+ long-baseline neutrino oscillation experiments. The experiments consisted of a Near Detector located $1.04 \mathrm{~km}$ from the NuMI beam target at Fermilab, Illinois, and a Far Detector located $734 \mathrm{~km}$ further in the Soudan Underground Mine, Minnesota. Both detectors were magnetized steel-scintillator sampling-tracking calorimeters positioned on the NuMI beamline axis. The detector pictures and bottom drawings are taken from Ref. [81]. . . . . . . . . . . 
3.4 The structure of a MINOS scintillator strip (top) and an illustration of the readout of a scintillator plane (bottom). Scintillation light produced by an ionizing particle is reflected inside the strip by the $0.25 \mathrm{~mm}$-thick outer reflective coating. When the light is absorbed, the WLS fiber reemits it isotropically. The wavelength-shifted photons with a direction within the total internal reflection cones are transported along the fiber toward the edges of the scintillator strip. The light is then carried by a clear optical fiber to a pixel of the PMT. These figures are taken from Ref. [81] and were made by M. Proga. . . . . . . .

3.5 Feynman diagram of a CC interaction between a $v_{\mu}$ and iron nucleus (top) and an example of a simulated event display in the MINOS detectors (bottom). The colored dots represents the magnitude of the signals in the scintillator strips and whether they have been reconstructed as part of a muon track or a shower. The outgoing muon forms a long track used to identify the CC interaction. The curvature of the track is caused by the magnetic field in the detector and is used to determine the electric charge sign of the muon. These figures are taken from Ref. [97]. . . . . . . . . . . . . . . . .

3.6 Feynman diagram of an NC interaction between a $v_{\alpha}$ and iron nucleus (top) and an example of a simulated event display in the MINOS detectors (bottom). The colored dots represents the magnitude of the signals in the scintillator strips and whether they have been reconstructed as part of a muon track or a shower. There are typically no long tracks in an NC event. These figures are taken from Ref. [97]. . . . . . . . . . . . . .

3.7 The MINOS and MINOS+ accelerator beam neutrino data. (Left) The data in terms of protons on target collected by MINOS and MINOS+ from March 2005 until July 2016. The data exposures used for the Far-over-Near and two-detector LED analyses presented in this dissertation are indicated at the bottom. (Right) The full CC MINOS dataset and the first two years of MINOS+ data (black points) as a function of reconstructed neutrino energy at the FD. The data is compared to the three-flavor paradigm prediction (blue line) and the no oscillations prediction (red line). The simulated contributions of the MINOS and MINOS+ CC samples are shown by the magenta and cyan hatched histograms, respectively. The right figure is taken from Ref. [98]. . . . . . . . . . . . . . . . . 
3.8 Long term behavior of the MINOS Near (green points) and Far (blue points) Detectors. (Top) The daily change in detector response normalized to the first data for each detector. (Middle) The measured PMT gains, averaged over three day intervals. (Bottom) The relative light level, defined as the ratio of the detector response to the PMT gains, normalized to the first data for each detector. . . . . . . . . . . . . .

3.9 (Top) The average time difference between East and West strip end recordings of through-going cosmic muon hits as a function of the plane number in the Far Detector, before and after timing calibration is applied, for a six month period in 2014. For each plane the time differences are averaged across all strips within that plane. The piecewise pattern observed before calibration is applied is due to the readout system setup. (Bottom) The distribution of calibrated East-West differences for all strips, which follows a Gaussian distribution with standard deviation of $0.40 \mathrm{~ns}$. These figures are taken from Ref. [102] . . . . . . .

3.10 The distribution of timing calibration constants for all Far Detector strip ends for different one year intervals. This figure is taken from Ref. [102]. . . . . . . . . . . . . . .

3.11 Muon energy loss in MINOS polystyrene scintillator. (Top) Energy loss per scintillator plane along the muon tracks [103]. (Bottom) Stopping power or mean energy loss per distance of stopping muons in the Far Detector as a function of momentum $[103] \ldots \ldots \ldots \ldots \ldots \ldots$

3.12 The effect of the calibration procedure on the detector response to cosmic muons for Far Detector (top panels) and Near Detector (bottom panels) planes as a function of the vertical and horizontal position during the MINOS and MINOS+ eras. Midcalibration refers to the stage between strip-to-strip and attenuation calibration. . . . . . . . . . . .

3.13 The calibrated response of the detectors as a function of time for the ND (left) and FD (right) during the three years of MINOS+ operation, run periods (RP) XI through XIII. The ratio of MEU to MEU DB shows the fractional change in MEU w.r.t. the constant values used throughout the MINOS and MINOS+ eras. 77

3.14 The reconstructed neutrino (left) and antineutrino (right) energy spectra at the ND from the first MINOS run period for data (black points) and MC simulation, before (blue line) and after (red line) applying the SKZP weights. This figure is taken from Ref. [110]. . . . . . . . . . . . . 
3.15 Distributions of the three $k \mathrm{NN}$ shower variables (top left, bottom left, and top right) and the true shower energy distributions (bottom right) for MINOS and MINOS+. These figures are taken from Ref. [45]. . . . . . . . . . . . . . .

3.16 The MINOS+ intensity effect. (Left) The ND CC reconstructed energy spectrum for the second year of MINOS+ data taking. A dip is observed in the peak bins for the months March through May 2015. This figure is taken from Ref. [46]. (Right) The intensity effect is observed as a drop in the calibrated response of the ND as a function of time when using stopping muons originating from NuMI neutrino events (red points) to calculate the MEU value. . . . . . . . . . . . . . . . . . . .

4.1 Preselection requirements to remove poorly reconstructed events in the ND. The maximum number of consecutive planes with energy deposits is required to be larger than three (top row), and the pulse height fraction needs to be larger than 50\% (bottom row). The event selection is shown for both the first two years of MINOS+ only (left column) and with the MINOS sample added (right column). Data (black points) are compared to unoscillated MC simulation (red line) and the fraction of poorly reconstructed events is shown (hatched histogram). The blue arrow indicates the region of the plot for which events are accepted by the ND preselection. These figures are taken from Ref. [120]. . . . . . . . . . . . . . . . .

4.2 Distribution of the event vertex coordinates in the ND. The $X Y$-view of the $\mathrm{NC}$ event vertices (top left), with the solid black shape representing the accepted region. The $X$ coordinate distribution (top right), the $Y$ coordinate distribution (bottom left), and the $Z$ coordinate distribution (bottom right) of ND data and MC events. These figures are taken from Ref. [120]. .

4.3 Distribution of the event vertex coordinates in the FD. The $X Y$-view of the NC event vertices (top left). The $X$ coordinate distribution (top right), the $Y$ coordinate distribution (bottom left), and the $Z$ coordinate distribution (bottom right) of FD data and $\mathrm{MC}$ events, assuming three-flavor oscillation. The drop in events around $Z=15 \mathrm{~m}$ corresponds to the gap between the two FD modules. These figures are taken from Ref. [120]. . 
4.4 Topology selection requirements to remove $\mathrm{CC}$ background events in the ND. The energy deposition cannot occur in more than 47 consecutive planes (top row), and the event cannot contain a track that extends more than 5 planes beyond the end of the shower (bottom row). The event selection is shown for both the first two years of MINOS+ only (left column) and with the MINOS sample added (right column). Data (black points) are compared to unoscillated MC simulation (red line) and the fraction of poorly reconstructed events is shown (hatched histogram). The blue arrow indicates the region of the plot for which events are accepted by the ND preselection. These figures are taken from Ref. [120]. . . . . . . . . . . . . .

4.5 Topology selection requirements to remove $\mathrm{CC}$ background events in the FD. The energy deposition cannot occur in more than 47 consecutive planes (top row), and the event cannot contain a track that extends more than 5 planes beyond the end of the shower (bottom row). The event selection is shown for both the first two years of MINOS+ only (left column) and with the MINOS sample added (right column). Data (black points) are compared to three-flavor MC simulation (red line) and the fraction of poorly reconstructed events is shown (hatched histogram). The blue arrow indicates the region of the plot for which events are accepted by the FD preselection. These figures are taken from Ref. [120]. . . . . . . . . . . . . .

4.6 The $X Y$-view of the distribution of the CC event vertex coordinates in the ND (left) and FD (right). These figures are taken from Ref. [120]. . . . . . . . . . . . . . . .

4.7 Distributions of the four $k \mathrm{NN}$ variables used to separate $\mathrm{CC}$ events from $\mathrm{NC}$ background events. An equal number of $\mathrm{CC}$ events and NC background events is used for the training set. These figures are taken from Ref. [45]. . . . . . . . . . . . . .

4.8 Distribution of roID for $k=80$ for the MINOS+ ND MC sample. This figure is taken from Ref. [45]. . . . . . . . . . . . . .

4.9 Purity and efficiency of the NC (top row) and CC (bottom row) selection procedures in the ND and FD as a function of energy. The purity and efficiency are shown for both the first two years of MINOS+ only (left column) and with the MINOS sample added (right column). These figures are taken from Ref. [120].

4.10 Simulated ND (top row) and FD (bottom row) event energy spectra for the CC (left column) and NC (right column) selected samples for MINOS only. The spectra are obtained assuming three-flavor oscillations. The main backgrounds are NC and CC $v_{\mu}$ events for the $\mathrm{CC}$ and $\mathrm{NC}$ samples, respectively. . . . . . . 
4.11 Simulated ND (top row) and FD (bottom row) event energy spectra for the CC (left column) and NC (right column) selected samples for MINOS+ only. The spectra are obtained assuming three-flavor oscillations. The main backgrounds are NC and CC $v_{\mu}$ events for the $\mathrm{CC}$ and $\mathrm{NC}$ samples, respectively. . . . . . .

4.12 Simulated ND (top row) and FD (bottom row) event energy spectra for the CC (left column) and NC (right column) selected samples for MINOS and MINOS+. The spectra are obtained assuming three-flavor oscillations. The main backgrounds are $\mathrm{NC}$ and $\mathrm{CC} \boldsymbol{v}_{\mu}$ events for the CC and NC samples, respectively. 103

5.1 The Far-over-Near ratio of the MINOS CC (top) and NC (bottom) data (black points) as a function of the reconstructed neutrino energy. The error bars represent statistical uncertainties. The three-flavor prediction is shown in red. The LED lowest $\chi^{2}$ prediction is shown with its systematic uncertainty (blue line and band), constructed, for illustration purposes, from the square roots of the diagonal elements of the systematic covariance matrix. . . . . . . . . . . . . . . . . . .

5.2 Corrections to the MC samples. (Top row) The effect of changing from an MC sample normalization by total data POT (red line) to a sample-by-sample normalization by data run period POT (blue line) on the CC (left) and NC (right) samples. (Bottom row) The effect of a $1.25 \mathrm{~cm}$ NuMI beam target $Z$ position shift on the CC (left) and NC (right) samples. . . . . . . . . .

5.3 A toy model example to illustrate the importance of using the correct statistical uncertainty in the dynamic scaling procedure, comparing static scaling for the three-flavor prediction (top left) to static scaling (top right), dynamic scaling using the LED ND prediction (bottom left), and dynamic scaling using the threeflavor ND prediction (bottom right) for the LED prediction. .

5.4 The total systematic uncertainty for the MINOS samples. The fractional uncertainty in the CC (top left) and NC (top right) Far-over-Near ratios and the corresponding covariance matrices for the CC (bottom left) and NC (bottom right) samples. . . .

5.5 The acceptance systematic uncertainty for the MINOS samples. The fractional uncertainty in the CC (top left) and NC (top right) Far-over-Near ratios and the corresponding covariance matrices for the CC (bottom left) and NC (bottom right) samples.123

5.6 The normalization systematic uncertainty for the MINOS samples. The fractional uncertainty in the CC (top left) and NC (top right) Far-over-Near ratios and the corresponding covariance matrices for the $\mathrm{CC}$ (bottom left) and $\mathrm{NC}$ (bottom right) samples. . . . . . . . . . . . . . . . 
5.7 The NC selection systematic uncertainty for the MINOS sample. The fractional uncertainty in the NC Far-over-Near ratio (left) and the corresponding covariance matrix (right). . . . .

5.8 The energy scale systematic uncertainties for the MINOS samples. The fractional uncertainty in the CC (top left) and $\mathrm{NC}$ (top right) Far-over-Near ratios and the corresponding covariance matrices for the $\mathrm{CC}$ (bottom left) and $\mathrm{NC}$ (bottom right) samples. . . . . . . . . . . . . . . .

5.9 The neutrino cross section systematic uncertainty for the MINOS samples. The fractional uncertainty in the CC (top left) and NC (top right) Far-over-Near ratios and the corresponding covariance matrices for the CC (bottom left) and NC (bottom right) samples. . . . . . . . . . . . .

5.10 The background systematic uncertainty for the MINOS samples. The fractional uncertainty in the CC (top left) and $\mathrm{NC}$ (top right) Far-over-Near ratios and the corresponding covariance matrices for the $\mathrm{CC}$ (bottom left) and $\mathrm{NC}$ (bottom right) samples. . . . . . . . . . . . . .

5.11 The hadron production systematic uncertainty for the MINOS samples. The fractional uncertainty in the CC (top left) and $\mathrm{NC}$ (top right) Far-over-Near ratios and the corresponding covariance matrices for the CC (bottom left) and NC (bottom right) samples. . . . . . . . . . . . . .

5.12 The beam optics systematic uncertainty for the MINOS samples. The fractional uncertainty in the $\mathrm{CC}$ (top left) and $\mathrm{NC}$ (top right) Far-over-Near ratios and the corresponding covariance matrices for the CC (bottom left) and NC (bottom right) samples. . . . . . . . . . . . . . .

5.13 The sensitivity surfaces for the LED model in the $\left(R, m_{0}\right)$ plane for a simulated MINOS exposure of $10.56 \times 10^{20}$ POT and assuming three-flavor simulated data: the $\chi^{2}$ surface (top left), the $\Delta \chi^{2}$ surface (top right), and the best fit values for $\Delta m_{32}^{2}$ (bottom left) and $\theta_{23}$ (bottom right). The white region in the bottom left panel corresponds to $\Delta m_{32}^{2}$ values between $10^{-6}$ and $1.4 \times 10^{-3} \mathrm{eV}^{2}$. . . . . . . . . . . . . . .

5.14 The data surfaces for the LED model in the $\left(R, m_{0}\right)$ plane for the MINOS exposure of $10.56 \times 10^{20}$ POT: the $\chi^{2}$ surface (top left), the $\Delta \chi^{2}$ surface (top right), and the best fit values for $\Delta m_{32}^{2}$ (bottom left) and $\theta_{23}$ (bottom right). The white region in the bottom left panel corresponds to $\Delta m_{32}^{2}$ values between $10^{-6}$ and $1.4 \times 10^{-3} \mathrm{eV}^{2}$. 
5.15 Illustration of the difference between the global scan (top right) and raster scan (bottom right) 90\% C.L. limits for a simple two-dimensional toy $\chi^{2}$ surface (left). . . . . . . . . .

5.16 Interpreting the sensitivity and data $\chi^{2}$ surfaces for the MINOS exposure of $10.56 \times 10^{20}$ POT: the cumulative effect of the systematic uncertainties on the global $90 \%$ C.L. $\left(\Delta \chi^{2}=4.61\right)$ Asimov sensitivity coverage (top left), comparing the global $90 \%$ C.L. $\left(\Delta \chi^{2}=4.61\right)$ Asimov sensitivity and data coverage (top right), and the Feldman-Cousins $90 \%$ C.L. data coverage (bottom left) and its interpretation in the $\sin ^{2} \theta_{\text {eff }}^{\mu \mu}$ plane (bottom right). . . . . . . . . . . . . . .

5.17 The $90 \%$ up value surface of the $\Delta \chi_{\mathrm{FC}}^{2}$ distributions used to construct the MINOS Far-over-Near Feldman-Cousins $90 \%$ C.L. data coverage in Fig. 5.16. The global 90\% C.L. data coverage (blue line) is shown for reference. . . . . . . . . . . .

5.18 The $\Delta \chi_{\mathrm{FC}}^{2}$ and $\chi^{2}$ profiles (top rows) and the best fit distributions (middle and bottom rows) of the LED model parameters for 500 fluctuated simulated experiments assuming the true values $R=0.089 \mu \mathrm{m}, m_{0}=0.291 \mathrm{eV}, \Delta m_{32}^{2}=2.71 \times 10^{-3} \mathrm{eV}^{2}$, and $\theta_{23}=0.679 \ldots \ldots \ldots \ldots \ldots$

5.19 The $\Delta \chi_{\mathrm{FC}}^{2}$ and $\chi^{2}$ profiles (top rows) and the best fit distributions (middle and bottom rows) of the LED model parameters for 500 fluctuated simulated experiments assuming the true values $R=0.513 \mu \mathrm{m}, m_{0}=0.006 \mathrm{eV}, \Delta m_{32}^{2}=2.43 \times 10^{-3} \mathrm{eV}^{2}$, and $\theta_{23}=0.702 \ldots \ldots \ldots \ldots \ldots$

5.20 A fluctuated sensitivity study. (Top) The effect of the atmospheric parameters $\Delta m_{32}^{2}$ and $\theta_{23}$ on the MINOS Far-over-Near global 90\% C.L. Asimov sensitivity and data coverages. (Bottom) A comparison of the MINOS Far-over-Near global 90\% C.L. Asimov and median fluctuated sensitivity coverages, assuming fixed atmospheric parameters. . . . . . . . . . .

5.21 The Far-over-Near ratio of the MINOS and MINOS+ CC (top) and NC (bottom) data (black points) as a function of the reconstructed neutrino energy. The error bars represent statistical uncertainties. The three-flavor prediction is shown in red. The LED lowest $\chi^{2}$ prediction is shown with its systematic uncertainty (blue line and band), constructed, for illustration purposes, from the square roots of the diagonal elements of the systematic covariance matrix. . . . . . . . . . . 
5.22 The flux modification systematic uncertainty for the MINOS/MINOS+ samples. The fractional uncertainty in the CC (top left) and $\mathrm{NC}$ (top right) Far-over-Near ratios and the corresponding covariance matrices for the $\mathrm{CC}$ (bottom left) and $\mathrm{NC}$ (bottom right) samples. . . . . . . . . . . . . . .

5.23 The total systematic uncertainty for the MINOS/MINOS+ samples. The fractional uncertainty in the $\mathrm{CC}$ (top left) and $\mathrm{NC}$ (top right) Far-over-Near ratios and the corresponding covariance matrices for the $\mathrm{CC}$ (bottom left) and $\mathrm{NC}$ (bottom right) samples. . . . . . . . . . . . . . . .

5.24 The sensitivity surfaces for the LED model in the $\left(R, m_{0}\right)$ plane for a simulated MINOS/MINOS+ exposure of $16.36 \times 10^{20} \mathrm{POT}$ and assuming three-flavor simulated data: the $\chi^{2}$ surface (top left), the $\Delta \chi^{2}$ surface (top right), and the best fit values for $\Delta m_{32}^{2}$ (bottom left) and $\theta_{23}$ (bottom right). . . . . . .

5.25 The data surfaces for the LED model in the $\left(R, m_{0}\right)$ plane for the MINOS/MINOS+ exposure of $16.36 \times 10^{20}$ POT: the $\chi^{2}$ surface (top left), the $\Delta \chi^{2}$ surface (top right), and the best fit values for $\Delta m_{32}^{2}$ (bottom left) and $\theta_{23}$ (bottom right). . . . .

5.26 Comparison of the Far-over-Near global 90\% C.L Asimov sensitivity and data coverages for the MINOS samples only and for the MINOS/MINOS+ samples. . . . . . . . . . . .

6.1 Correcting the FLUGG neutrino flux prediction. (Top row) Optimizing the FLUKA hadron production weights by performing a simultaneous fit to the simulated neutrino flux from $\pi^{+}$ neutrino parents corrected using the PPFX prediction in the $0-20 \mathrm{GeV}$ range (left) and a MINOS+ ND data sample in the $40-60 \mathrm{GeV}$ range (right), with the magnetic horns disabled in both cases to remove any effects related to beam focusing. (Bottom) The reconstructed neutrino neutrino energy spectrum at the ND for MINOS+ ND data with the magnetic horns turned on (black points) and MC simulation before (blue line) and after (red line) applying the hadron production weights obtained from the fits shown in the top row panels. These figures are taken from Ref. [140]. . . . . . . . . . . . . . . . .

6.2 The structure of the two-detector covariance matrix for the CC sample. It should be noted that the $(1,1)$ bin of the matrix corresponds to the bottom left corner in this figure. A similar matrix structure is used for the $\mathrm{NC}$ sample. . . . . . . . . 
6.3 Comparison of the ND (left) and FD (right) LED two-detector predictions at two points in the LED parameter space obtained through exact calculation (green line) or through multilinear interpolation between predictions calculated for neighboring LED grid points (blue line). . . . . . . . . . . . .

6.4 The total systematic uncertainty for the MINOS/MINOS+ samples. The fractional uncertainty in the ND CC and NC (top left and right) and FD CC and NC (middle left and right) samples and the corresponding two-detector covariance matrices for the CC (bottom left) and $\mathrm{NC}$ (bottom right) samples. . . . . . . .

6.5 The acceptance systematic uncertainty for the MINOS/MINOS+ samples. The fractional uncertainty in the ND CC and NC (top left and right) samples and the corresponding two-detector covariance matrices for the $\mathrm{CC}$ (bottom left) and $\mathrm{NC}$ (bottom right) samples. . . . . . . . . . . . . . . .

6.6 The normalization systematic uncertainty for the MINOS/MINOS+ samples. The fractional uncertainty in the ND CC and NC (top left and right) and FD CC and $\mathrm{NC}$ (middle left and right) samples and the corresponding two-detector covariance matrices for the $\mathrm{CC}$ (bottom left) and $\mathrm{NC}$ (bottom right) samples. . . . . . 176

6.7 The NC selection systematic uncertainty for the MINOS/MINOS+ samples. The fractional uncertainty in the ND NC (top left) and FD NC (bottom left) samples and the corresponding twodetector covariance matrix for the NC (right) samples. . . . . 177

6.8 The energy scale systematic uncertainties for the MINOS/MINOS+ samples. The fractional uncertainty in the ND CC and NC (top left and right) and FD CC and NC (middle left and right) samples and the corresponding two-detector covariance matrices for the $\mathrm{CC}$ (bottom left) and $\mathrm{NC}$ (bottom right) samples. . . . . .

6.9 The neutrino cross section systematic uncertainty for the MINOS/MINOS+ samples. The fractional uncertainty in the ND $\mathrm{CC}$ and $\mathrm{NC}$ (top left and right) and FD CC and NC (middle left and right) samples and the corresponding two-detector covariance matrices for the $\mathrm{CC}$ (bottom left) and $\mathrm{NC}$ (bottom right) samples. . . . . . . . . . . . . . . . . . 180

6.10 The background systematic uncertainty for the MINOS/MINOS+ samples. The fractional uncertainty in the ND CC and NC (top left and right) and $\mathrm{FD} \mathrm{CC}$ and $\mathrm{NC}$ (middle left and right) samples and the corresponding two-detector covariance matrices for the CC (bottom left) and NC (bottom right) samples. . . . . . 182

xxix 
6.11 The hadron production systematic uncertainty for the MINOS/MINOS+ samples. The fractional uncertainty in the ND CC and NC (top left and right) and FD CC and NC (middle left and right) samples and the corresponding two-detector covariance matrices for the CC (bottom left) and NC (bottom right) samples. . . . . . 183

6.12 The run period weight systematic uncertainty for the MINOS/MINOS+ samples. The fractional uncertainty in the ND CC and NC (top left and right) and FD CC and $\mathrm{NC}$ (middle left and right) samples and the corresponding two-detector covariance matrices for the CC (bottom left) and NC (bottom right) samples. . . . . . 185

6.13 The beam optics systematic uncertainty for the MINOS/MINOS+ samples. The fractional uncertainty in the ND CC and NC (top left and right) and FD CC and NC (middle left and right) samples and the corresponding two-detector covariance matrices for the CC (bottom left) and NC (bottom right) samples. . . . . .

6.14 The sensitivity surfaces for the LED model in the $\left(R, m_{0}\right)$ plane for a simulated MINOS and MINOS+ exposure of $16.36 \times 10^{20}$ POT and assuming three-flavor simulated data: the $\chi^{2}$ surface (top left), the $\Delta \chi^{2}$ surface (top right), and the best fit values for $\Delta m_{32}^{2}$ (bottom left) and $\theta_{23}$ (bottom right). The white region in the bottom left panel corresponds to $\Delta m_{32}^{2}$ values between $10^{-6}$ and $1.4 \times 10^{-3} \mathrm{eV}^{2}$. . . . . . . . . . . . . .

6.15 The data surfaces for the LED model in the $\left(R, m_{0}\right)$ plane for a MINOS and MINOS+ exposure of $16.36 \times 10^{20}$ POT: the $\chi^{2}$ surface (top left), the $\Delta \chi^{2}$ surface (top right), and the best fit values for $\Delta m_{32}^{2}$ (bottom left) and $\theta_{23}$ (bottom right). The white region in the bottom left panel corresponds to $\Delta m_{32}^{2}$ values between $10^{-6}$ and $1.4 \times 10^{-3} \mathrm{eV}^{2}$. . . . . . . . . . . . .

6.16 Comparison of the global minimum LED prediction (blue line) and three-flavor prediction (red line) to the data (black points) in the ND (left) and FD (right). The ratio of the data and global minimum LED prediction to the three-flavor prediction (top row) and the cumulative $\chi^{2}$ profiles of the global minimum LED and three-flavor predictions (bottom row) are also shown. It should be noted that the global minimum LED prediction is almost identical to the three-flavor prediction. . . . . . . . . . 
6.17 The effect of the individual samples and atmospheric parameters on the global $90 \%$ C.L. sensitivity coverages for a simulated MINOS and MINOS+ exposure of $16.36 \times 10^{20}$ POT and assuming three-flavor simulated data: the CC versus $\mathrm{NC}$ samples (top left), a two-detector versus a single-detector fit (top right), the four individual samples (bottom left), and the effect of fixing the atmospheric parameters (bottom right). The bottom right panel also shows the comparison between the global $90 \%$ C.L. sensitivity and data coverages. . . . . . . . . . . . . . .

6.18 (Top left) The effect of the individual systematic uncertainties on the global 90\% C.L. sensitivity coverage for a simulated MINOS and MINOS+ exposure of $16.36 \times 10^{20}$ POT and assuming three-flavor simulated data. (Top right) Global 90\% C.L. sensitivity coverages assuming four different simulated LED signals (indicated by the stars). (Bottom left) Comparison of the two-detector global 90\% C.L. sensitivity coverages to their Farover-Near counterparts. (Bottom right) Comparison of the twodetector raster, global, and Feldman-Cousins 90\% C.L. data coverages. . . . . . . . . . . . . . .

6.19 The $90 \%$ up value surface of the $\Delta \chi_{\mathrm{FC}}^{2}$ distributions used to construct the MINOS/MINOS+ two-detector Feldman-Cousins 90\% C.L. data coverage and sensitivity bands in Fig. 6.23. The global $90 \%$ C.L. data coverage (blue line) is shown for reference. 197

6.20 The $\Delta \chi_{\mathrm{FC}}^{2}$ and $\chi^{2}$ profiles (top rows) and the best fit distributions (middle and bottom rows) of the LED model parameters for 600 simulated experiments assuming the values $R=$ $0.447 \mu \mathrm{m}, m_{0}=0.052 \mathrm{eV}$, and fluctuated values of the Asimov atmospheric parameters $\Delta m_{32}^{2}=2.37 \times 10^{-3} \mathrm{eV}^{2}$, and $\theta_{23}=0.695 .199$

6.21 The $\Delta \chi_{\mathrm{FC}}^{2}$ and $\chi^{2}$ profiles (top rows) and the best fit distributions (middle and bottom rows) of the LED model parameters for 600 simulated experiments assuming the values $R=$ $0.085 \mu \mathrm{m}, m_{0}=0.340 \mathrm{eV}$, and fluctuated values of the Asimov atmospheric parameters $\Delta m_{32}^{2}=2.37 \times 10^{-3} \mathrm{eV}^{2}$, and $\theta_{23}=0.695 .200$

6.22 A set of 100 statistically and systematically fluctuated simulated three-flavor spectra used to construct the $1 \sigma$ and $2 \sigma$ sensitivity bands in the $\left(R, m_{0}\right)$ plane shown in Fig. 6.23. The ND CC (top left), ND NC (top right), FD CC (bottom left), and FD NC (bottom right) spectra are shown. The ratio of the fluctuated simulated spectra (gray lines) to the Asimov spectrum (red line) is shown for each sample. Two example fluctuated simulated spectra are highlighted in cyan and magenta for illustrative purposes. . . . . . . . . . . . . 
6.23 A fluctuated sensitivity study. (Top) A comparison of the MINOS/MINOS+ two-detector global 90\% C.L. Asimov (red line) and median fluctuated (orange line) sensitivity coverages. (Bottom) The MINOS/MINOS+ two-detector Feldman-Cousins 90\% C.L. data coverage (blue line) is overlaid on the $1 \sigma$ and $2 \sigma$ sensitivity bands constructed from the Feldman-Cousins $90 \%$ C.L. interpretations of the $\Delta \chi^{2}$ surfaces that are used to obtain the global $90 \%$ C.L. fluctuated sensitivity coverages shown in the top panel. . . . . . . . . . . . . . . .

6.24 Interpreting the MINOS/MINOS+ two-detector Feldman-Cousins $90 \%$ C.L. data limit in the $\left(R, m_{0}\right)$ plane in terms of the effective mixing angle $\sin ^{2} \theta_{\text {eff }}^{\mu \mu}$ (top) and the mass squared splitting

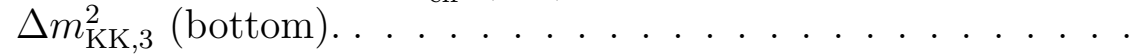

A.1 Fluctuated simulated data spectra in the two-detector analysis. The full analysis systematic covariance matrices for the CC (top left) and NC (top right) samples used in the multivariate Gaussian fluctuation procedure through SVD+QR upper-triangular matrix decomposition. A set of 30 fluctuated spectra (bottom left) and their ratio to the nominal spectrum (bottom right) are shown for the four samples, ND CC, ND NC, FD CC, and FD NC. . . . . . . . . . . . . . . . .

A.2 The original matrices from Fig. A.1 (top row) and the fractional difference of the original and reconstructed matrices for the Cholesky (middle row) and SVD+QR (bottom row) decomposition obtained from 10000 fluctuated spectra. Unlike in Fig. A.1, the matrices are shown in terms of energy bins rather than reconstructed energy. The left (right) column shows the CC (NC) matrix. . . . . . . . . . . . . . . . .

A.3 The effect of increasing the number of fluctuated simulated data spectra on the fractional difference between the original and reconstructed full analysis systematic covariance matrix for the Cholesky decomposition: 1000 spectra (top row), 10000 spectra (middle row), 100000 spectra (bottom row), with the left (right) column showing the $\mathrm{CC}(\mathrm{NC})$ matrix. . . . . . . . . . .

A.4 Fluctuated simulated data spectra in the two-detector analysis. The energy scale systematic covariance matrices for the CC (top left) and NC (top right) samples used in the multivariate Gaussian fluctuation procedure through SVD+QR upper-triangular matrix decomposition. A set of 30 fluctuated spectra (bottom left) and their ratio to the nominal spectrum (bottom right) are shown for the four samples, ND CC, ND NC, FD CC, and FD NC. . . . . . . . . . . . . . 
A.5 The original matrices from Fig. A.4 (top row) and the fractional difference of the original and reconstructed matrices for the SVD+QR (bottom row) decomposition obtained from 10000 fluctuated spectra. Unlike in Fig. A.4, the matrices are shown in terms of energy bins rather than reconstructed energy. The left (right) column shows the CC (NC) matrix. . . . . . . . .

A.6 Fluctuated simulated data spectra in the two-detector analysis. The hadron production systematic covariance matrices for the $\mathrm{CC}$ (top left) and NC (top right) samples used in the multivariate Gaussian fluctuation procedure through SVD+QR uppertriangular matrix decomposition. A set of 30 fluctuated spectra (bottom left) and their ratio to the nominal spectrum (bottom right) are shown for the four samples, ND CC, ND NC, FD CC, and FD NC. . . . . . . . . . . . . . . . . .

A.7 The original matrices from Fig. A.6 (top row) and the fractional difference of the original and reconstructed matrices for the SVD+QR (bottom row) decomposition obtained from 10000 fluctuated spectra. Unlike in Fig. A.6, the matrices are shown in terms of energy bins rather than reconstructed energy. The left (right) column shows the CC (NC) matrix. . . . . . . .

A.8 Fluctuated simulated data spectra in the two-detector analysis. The cross section systematic covariance matrices for the CC (top left) and NC (top right) samples used in the multivariate Gaussian fluctuation procedure through SVD+QR upper-triangular matrix decomposition. A set of 30 fluctuated spectra (bottom left) and their ratio to the nominal spectrum (bottom right) are shown for the four samples, ND CC, ND NC, FD CC, and FD NC. . . . . . . . . . . . . . . . .

A.9 The original matrices from Fig. A.8 (top row) and the fractional difference of the original and reconstructed matrices for the SVD+QR (bottom row) decomposition obtained from 10000 fluctuated spectra. Unlike in Fig. A.8, the matrices are shown in terms of energy bins rather than reconstructed energy. The left (right) column shows the $\mathrm{CC}(\mathrm{NC})$ matrix. . . . . . . . . .

B.1 The Asimov and median fluctuated global 90\% C.L. sensitivity coverages for the two-detector fit method, with 200 fluctuated sensitivity coverages calculated for the full analysis framework (left) and 100 fluctuated sensitivity coverages generated for the case where the atmospheric parameters $\Delta m_{23}^{2}$ and $\theta_{23}$ are fixed to their global fit values (both when generating the fluctuated spectra and in the fit) and with $\theta_{34}$ fixed to 0 (right), as is assumed for the purpose of this study. . . . . . . . 
B.2 The global 90\% C.L. Asimov sensitivity coverages for the twodetector fit method compared with the Near Detector and Far Detector independent contributions. Sensitivity coverages are shown for the joint CC and NC-selected samples (left), for the isolated CC-selected sample (middle), and for the isolated NCselected sample (right). These figures are taken from Ref. [146].

B.3 The CC covariance matrices used for this study, for the full analysis systematic uncertainties (left) and for a simple fully correlated $5 \%$ normalization uncertainty (right). The bottom left and top right quadrants correspond to the Far and Near Detector systematic uncertainties, respectively. The other quadrants contain the correlations between both detectors. . . . . . . . .

B.4 The effect of fixing the atmospheric parameters $\Delta m_{32}^{2}$ and $\theta_{23}$ in the MINOS/MINOS+ two-detector $3+1$ sterile fit on the global 90\% C.L. Asimov sensitivity coverage, assuming normal ordering (left) and inverted ordering (right). These figures are taken from Ref. [147]. . . . . . . . . . . . . . . . . .

B.5 An ND CC sample study. The effect on the Asimov and fluctuated global 90\% C.L. sensitivity coverages for the ND CC sample when using, both for creating the fluctuated simulated data spectra and calculating the $\chi^{2}$ values, the full analysis systematic and statistical uncertainties (top row), the full analysis systematic and statistical uncertainties with correlations set to zero (middle row), and the statistical uncertainties only (bottom row). This is done for the full ND CC sample (left column) and for a scaled down sample normalized to the total number of FD events (right column). . . . . . . . . . . . . . .

B.6 An ND CC sample study. The effect on the Asimov and fluctuated global 90\% C.L. sensitivity coverages for the ND CC sample when using, both for creating the fluctuated simulated data spectra and calculating the $\chi^{2}$ values, the full analysis systematic and statistical uncertainties for different fractions of the full correlations. . . . . . . . . . . . . . . .

B.7 An ND CC sample study. A selection of simulated data spectra highlighted in color (right column) and their corresponding global 90\% C.L. sensitivity coverages (left column) for the ND CC sample when using, both for creating the fluctuated simulated data spectra and calculating the $\chi^{2}$ values, the full analysis systematic and statistical uncertainties (top row), the full analysis systematic and statistical uncertainties with correlations set to zero (middle row), and the statistical uncertainties only (bottom row). The left column panels are the same as the left column panels in Fig. B.5. . . . . . . . . . . . 
B.8 An ND CC sample study. The $\Delta \chi^{2}$ profiles at $\Delta m_{41}^{2}=500 \mathrm{eV}^{2}$ (middle row) and $\Delta m_{41}^{2}=5 \mathrm{eV}^{2}$ (bottom row) for the ND CC sample when using, both for creating the fluctuated simulated data spectra and calculating the $\chi^{2}$ values, the statistical uncertainties only (left column) and the full analysis systematic and statistical uncertainties (right column). The crossings of the $\Delta \chi_{\text {Global }}^{2}=4.61$ lines (cyan) with the $\Delta \chi^{2}$ profiles give the global $90 \%$ C.L. values of $\sin ^{2}\left(\theta_{24}\right)$ for the respective $\Delta m_{41}^{2}$ value (top row). Similarly, the crossings with the $\Delta \chi_{\mathrm{FC}}^{2}$ lines (magenta) give the Feldman-Cousins $90 \%$ C.L. values of $\sin ^{2}\left(\theta_{24}\right)$.

B.9 The ratio of the four-flavor and three-flavor $v_{\mu}$ survival probabilities at the MINOS Near Detector (assuming an oscillation distance of $1 \mathrm{~km}$ ) at $\Delta m_{41}^{2}=500 \mathrm{eV}^{2}$ (top left), $5 \mathrm{eV}^{2}$ (middle left) and $0.1 \mathrm{eV}^{2}$ (bottom left) for different values of $\theta_{24}$. The right column shows the ratio of the four-flavor and three-flavor $v_{\mu}$ survival probabilities divided by $1-\sin ^{2}\left(2 \theta_{24}\right)$ to remove the normalization shift induced by rapid sterile oscillations. These figures are taken from Ref. [146]. . . . . . . . . . . . . . . .

B.10 The ratio of the four-flavor and three-flavor $v_{\mu}$ survival probabilities at the MINOS Far Detector (assuming an oscillation distance of $1 \mathrm{~km}$ ) at $\Delta m_{41}^{2}=500 \mathrm{eV}^{2}$ (top left), $5 \mathrm{eV}^{2}$ (middle left) and $0.1 \mathrm{eV}^{2}$ (bottom left) for different values of $\theta_{24}$. The right column shows the ratio of the four-flavor and three-flavor $v_{\mu}$ survival probabilities divided by $1-\sin ^{2}\left(2 \theta_{24}\right)$ to remove the normalization shift induced by rapid sterile oscillations. These figures are taken from Ref. [146]. . . . . . . . . . . . . .

B.11 A fully correlated $5 \%$ normalization uncertainty study. A selection of simulated data spectra highlighted in color (right column) and their corresponding global 90\% C.L. sensitivity coverages (left column) for the ND CC sample (top row), the FD CC sample (middle row), and the ND and FD CC samples (bottom row). The full analysis systematic and statistical uncertainties are used as the uncertainties for the $3+1$ sterile model predictions in the $\chi^{2}$ calculations. . . . . . . . . . . . .

B.12 A fully correlated $5 \%$ normalization uncertainty study. The effect on the Asimov and fluctuated global 90\% C.L. sensitivity coverages for the ND CC sample when using the fully correlated 5\% normalization uncertainties with different fractions of the full correlations for creating the fluctuated simulated data spectra and using the full analysis systematic and statistical uncertainties for calculating the $\chi^{2}$ values. . . . . . . . . 
B.13 A fully correlated 5\% normalization uncertainty study. The effect of shape and normalization of the fluctuated spectra on the fluctuated global 90\% C.L. sensitivity coverages for the ND CC sample when using the fully correlated $5 \%$ normalization uncertainties, with different fractions of the full correlations for creating the fluctuated simulated data spectra, and using the full analysis systematic and statistical uncertainties for calculating the $\chi^{2}$ values. . . . . . . . . . . . . .

B.14 A fully correlated 5\% normalization uncertainty study. The effect on the Asimov and fluctuated global 90\% C.L. sensitivity coverages for the ND CC sample when using the fully correlated $5 \%$ normalization uncertainties, both for creating the fluctuated simulated data spectra and calculating the $\chi^{2}$ values, for different fractions of the full correlations. . . . . . . . . . . .

B.15 A fully correlated 5\% normalization uncertainty study. The effect of shape and normalization of the fluctuated spectra on the fluctuated global 90\% C.L. sensitivity coverages for the ND CC sample when using the fully correlated $5 \%$ normalization uncertainties for creating the fluctuated simulated data spectra and using the fully correlated $5 \%$ normalization uncertainties with different fractions of the full correlations for calculating the $\chi^{2}$ values. . . . . . . . . . . . . . . . . . . . . . . . . 249 


\section{Chapter 1}

\section{Neutrino Oscillations}

Neutrinos are very light, spin- $\frac{1}{2}$, and electrically neutral elementary particles that are known to only interact through gravitation and the weak interaction. It is the combination of these properties that make them very hard to detect and study, despite their abundant presence in the Universe. Upon interacting through the weak interaction, neutrinos have a definite flavor, of which we know there are three: the electron neutrino $\left(v_{\mathrm{e}}\right)$, the muon neutrino $\left(v_{\mu}\right)$, and the tau neutrino $\left(v_{\tau}\right)$. These flavor eigenstates are mixtures of the neutrino mass eigenstates and thus have no definite mass. A consequence of this mixing and the small but non-vanishing masses of the neutrino mass eigenstates is the phenomenon of neutrino oscillations. This chapter starts with a brief introduction on neutrino physics, followed by an explanation of the neutrino oscillation formalism within the three-flavor paradigm. The last section of this chapter outlines the experimental motivations for neutrino oscillation searches beyond the three-flavor paradigm. The Large Extra Dimension model is discussed in detail in Chapter 2. 


\subsection{Neutrino Physics}

In 1911, Lise Meitner and Otto Hahn measured a continuous, rather than discrete, electron energy spectrum for electrons in nuclear beta decay [1], which at that time was thought to be a two-particle decay. In an attempt to preserve the conservation laws of energy, momentum, and spin, Wolfgang

Pauli postulated the existence of a new electrically neutral, spin- $\frac{1}{2}$, and nearly massless particle which interacts very weakly and carries away some of the energy in the nuclear beta decay [2]. In 1934, Enrico Fermi successfully modeled the nuclear beta decay at lower energies as an interaction at a single point of a neutron, a proton, an electron, and assuming the existence of this new particle, which he baptized the neutrino [3]

$$
\mathrm{n} \rightarrow \mathrm{p}+\mathrm{e}^{-}+\mathrm{v}
$$

Because neutrinos interact very weakly, it was not until 1956 that they were directly observed by Fred Reines and Clyde Cowan [4]. They detected electron antineutrinos $\left(\bar{v}_{\mathrm{e}}\right)$ coming from a nuclear reactor at the Savanah River Plant using two cadmium-doped water tanks sandwiched between three layers of scintillator surrounded by photomultiplier tubes. The antineutrinos originating from the reactor would undergo inverse beta decay

$$
\bar{v}_{\mathrm{e}}+\mathrm{p} \rightarrow \mathrm{n}+\mathrm{e}^{+}
$$

where the positron annihilated with an electron in the tank resulting in the back-to-back emission of photons and where the neutron was captured by 
cadmium resulting in a delayed photon. The temporal coincidence of these two photon signals in the scintillator was used to measure the inverse beta decay rate, which dropped significantly when turning off the nuclear reactor.

In 1962, Leon Lederman, Melvin Schwartz, and Jack Steinberger discovered the second type of neutrino [5], the muon neutrino, which was predicted by Bruno Pontecorvo [6] three years earlier. Employing the first accelerator neutrino beam at Brookhaven National Laboratory, muon neutrinos were created from the decay of pions resulting from the interactions of a $15 \mathrm{GeV}$ proton beam with a beryllium target. Using a spark chamber, it was observed that the neutrinos leading to muon signals are different from those that lead to electron signals.

The tau neutrino was observed for the first time in 2000 in the nuclear emulsion detectors of the DONUT [7] experiment at Fermilab, 25 years after it was postulated when the tau lepton was discovered [8].

No other neutrino species have been discovered since, which is consistent with the number of light active neutrino species determined from the $\mathrm{Z}$ boson decay width measurements at the SLAC Linear Collider (SLC) and at the Large Electron-Positron collider (LEP) at CERN [9]. From the comparison of the total width of the $\mathrm{Z}$ boson resonance to the width from the decays into visible particles, the decay width to neutrinos can be inferred. Assuming equal coupling of all neutrino species to the $\mathrm{Z}$ boson, the measurements yield $2.9840 \pm 0.0082$ light active neutrino types, as shown in Fig. 1.1, where light 
refers to a neutrino mass $m_{\mathrm{v}}<m_{\mathrm{Z}} / 2$ and active refers to the coupling with Standard Model (SM) [10-15] particles.

Combining Planck observations on the cosmic microwave background radiation with other astrophysical data gives $N_{\text {eff }}=2.91_{-0.37}^{+0.39}$ for the effective number of relativistic degrees of freedom, which is consistent with the value from the $\mathrm{Z}$ boson decay, and the sum of neutrino masses is constrained to $\sum_{i} m_{i}<(0.340-0.715) \mathrm{eV}[16]$. An overview of the constraints on the number of neutrino types from colliders, astrophysics, and cosmology is presented in Ref. [17].

The particle type, Dirac or Majorana, the absolute neutrino masses, and the mechanism that generates the neutrino masses are currently not known. If neutrinos are Dirac particles like all other fermions, then they have a defined chirality, with neutrinos always being left-handed and antineutrinos always being right-handed when assuming massless neutrinos. Chirality is a Lorentz invariant particle property that is related to helicity, and in the limit of small masses, chirality and helicity are the same. Helicity is defined as

$$
\frac{\mathbf{s} \cdot \mathbf{p}}{|\mathbf{s}||\mathbf{p}|}
$$

where $\mathbf{s}$ and $\mathbf{p}$ are the particle's spin and momentum, respectively. Hence, right-handedness corresponds to positive helicity and left-handedness to negative helicity. Antineutrinos were first observed to have positive helicity in the beta decay of polarized ${ }^{60}$ Co in 1956 [18], which also demonstrated parity violation in the weak interaction because helicity changes sign under parity trans- 


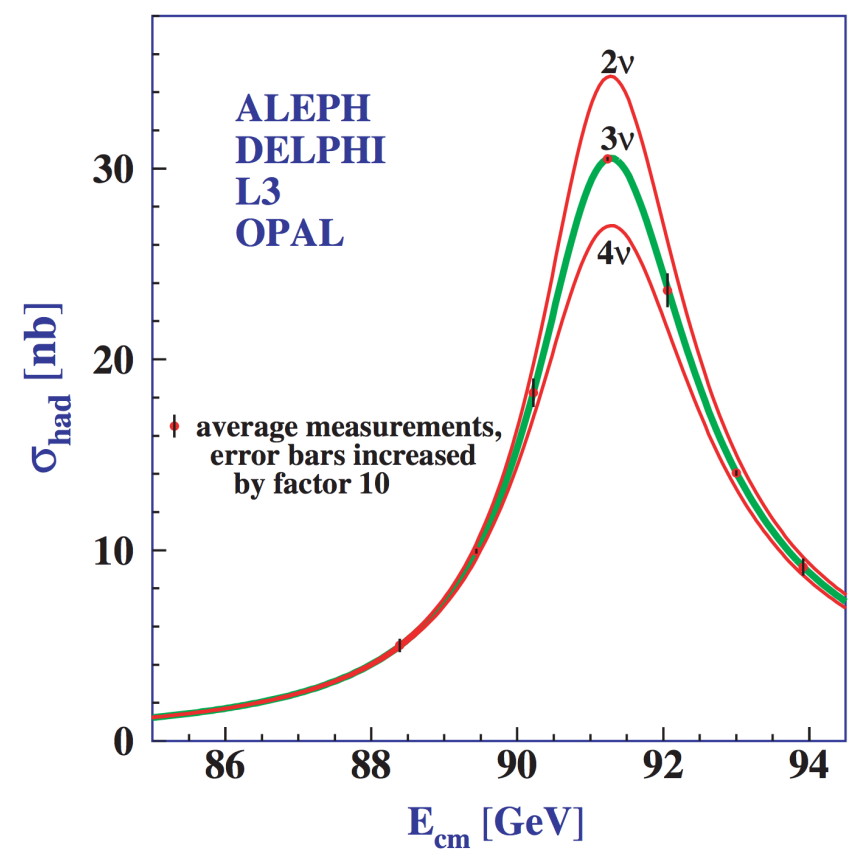

Figure 1.1: Measurements of the hadron production cross section around the $\mathrm{Z}$ boson resonance. The data included in this figure consist of 17 million $\mathrm{Z}$ decays accumulated by the ALEPH, DELPHI, L3 and OPAL experiments at LEP. The curves represent the predicted cross section for two, three, and four neutrino species with SM couplings and negligible mass. This figure is taken from Ref. [9].

formation. The negative helicity of neutrinos was first observed in 1958 [19]. Thus far, no right-handed neutrinos neutrinos or left-handed antineutrinos have been observed.

\subsection{Three-Flavor Neutrino Oscillations}

Neutrinos were originally assumed to be massless in the SM. Over the past decades, oscillations between the three known neutrino flavors have been 
observed through measurements of solar, atmospheric, reactor, and accelerator beam neutrinos. The observations of neutrino oscillations imply that neutrinos have non-zero masses. However, neutrino oscillation measurements only reveal information about the neutrino mass differences and do not allow an absolute mass measurement. Furthermore, it is not possible to determine the particle type, Dirac or Majorana, from neutrino oscillation measurements.

\subsubsection{Three-Flavor Formalism}

The formalism of neutrino oscillations was developed by Pontecorvo, Maki, Nakagawa, and Sakata [20-22]. Neutrinos only interact through the weak interaction (ignoring gravity) and upon doing so have a definite lepton flavor described by the flavor eigenstates $\left|v_{\mathrm{e}}\right\rangle,\left|v_{\mu}\right\rangle$, or $\left|v_{\tau}\right\rangle$. When propagating in vacuum, neutrinos have a definite mass described by the mass eigenstates $\left|v_{1}\right\rangle,\left|v_{2}\right\rangle$, or $\left|v_{3}\right\rangle$. The flavor and mass eigenstates are related by the unitary PMNS mixing matrix $U$, named after Pontecorvo, Maki, Nakagawa, and Sakata,

$$
\left|v_{\alpha}\right\rangle=\sum_{j} U_{\alpha j}^{*}\left|v_{j}\right\rangle
$$

with $\alpha=\mu$, e, or $\tau$ and $j=1,2,3$ in the case of three neutrino types. It should be noted that this summation can be extended to any number $N$ of neutrino types with $j=1, \ldots, N$. The time evolution of a flavor eigenstate is dictated by quantum mechanics to be

$$
\left|v_{\alpha}(t)\right\rangle=\sum_{j} U_{\alpha j}^{*} \exp \left(i p_{j} \cdot x\right)\left|v_{j}\right\rangle
$$


where $x$ and $p_{j}$ are the four-position $x=(t, \mathbf{x})$ of the neutrino and the fourmomentum $p_{j}=\left(E_{j}, \mathbf{p}_{\mathbf{j}}\right)$ of mass eigenstate $j$, respectively, assuming natural units with $c=1$. Assuming the mass eigenstates have the same threemomentum $\mathbf{p}$, the dot product $p_{j} \cdot x$ can be written as

$$
\begin{aligned}
p_{j} \cdot x & =E_{j} t-\mathbf{p} \cdot \mathbf{x} \\
& =t \sqrt{|\mathbf{p}|+m_{j}^{2}}-\mathbf{p} \cdot \mathbf{x} .
\end{aligned}
$$

Assuming highly relativistic neutrinos such that $m_{j} \ll E_{j}, t=L$, and $\mathbf{p} \cdot \mathbf{x}=|\mathbf{p}| L$, with $L$ the distance traveled by the neutrino, a binomial expansion can be performed such that

$$
\begin{aligned}
p_{j} \cdot x & =|\mathbf{p}| L\left(1+\frac{m_{j}^{2}}{2|\mathbf{p}|^{2}}\right)-|\mathbf{p}| L \\
& =\frac{m_{j} L}{2 E},
\end{aligned}
$$

where in the last step $|\mathbf{p}| \approx E$ is assumed, with $E$ the average of the $E_{i}$. Hence, the probability that a neutrino of flavor $\alpha$ has oscillated to a flavor $\beta$ after having traveled a distance $L$ is

$$
\begin{aligned}
P\left(v_{\alpha} \rightarrow v_{\beta}\right)= & \left|\left\langle v_{\beta} \mid v_{\alpha}(L)\right\rangle\right|^{2} \\
= & \left|\left(\sum_{k} U_{\beta k}\left\langle v_{k}\right|\right)\left(\sum_{j} U_{\alpha j}^{*} \exp \left(-\frac{m_{j} L}{2 E}\right)\left|v_{j}\right\rangle\right)\right|^{2} \\
= & \delta_{\alpha \beta}-4 \sum_{k>j} \operatorname{Re}\left[U_{\beta k} U_{\beta j}^{*} U_{\alpha k}^{*} U_{\alpha j}\right] \sin ^{2}\left(\frac{\Delta m_{k j}^{2} L}{4 E}\right) \\
& \quad+2 \sum_{k>j} \operatorname{Im}\left[U_{\beta k} U_{\beta j}^{*} U_{\alpha k}^{*} U_{\alpha j}\right] \sin \left(\frac{\Delta m_{k j}^{2} L}{2 E}\right),
\end{aligned}
$$


where $\Delta m_{k j}^{2}=m_{k}^{2}-m_{j}^{2}$ is the mass squared splitting between mass eigenstates $k$ and $j$.

In the three-flavor paradigm, the $3 \times 3$ mixing matrix $U$ can be parametrized by 3 mixing angles, $\theta_{12}, \theta_{13}$, and $\theta_{23}$, and one CP-violating phase, $\delta_{13}$ (referred to as $\delta_{\mathrm{CP}}$ in the remainder of this dissertation),

$$
U=\left(\begin{array}{ccc}
c_{12} c_{13} & c_{13} s_{12} & e^{-i \delta_{\mathrm{CP}}} s_{13} \\
-c_{23} s_{12}-e^{i \delta_{\mathrm{CP}}} c_{12} s_{13} s_{23} & c_{12} c_{23}-e^{i \delta_{\mathrm{CP}}} s_{12} s_{13} s_{23} & c_{13} s_{23} \\
s_{12} s_{23}-e^{i \delta_{\mathrm{CP}}} c_{12} c_{23} s_{13} & -e^{i \delta_{\mathrm{CP}}} c_{23} s_{12} s_{13}-c_{12} s_{23} & c_{13} c_{23}
\end{array}\right)
$$

where $s_{i j} \equiv \sin \left(\theta_{i j}\right)$ and $c_{i j} \equiv \cos \left(\theta_{i j}\right)$. Thus, the neutrino oscillation probabilities in vacuum, described by Formula (1.8), depend on the mixing parameters, the neutrino energy, the distance traveled by the neutrino (also called baseline in the context of neutrino oscillation experiments), and the neutrino mass squared splittings. It should be noted that the three-flavor oscillation probabilities can be further modified by matter effects when neutrinos travel through matter $[23,24]$. Electron neutrinos and antineutrinos undergo forward coherent scattering with electrons in the matter through which they propagate, which alters the neutrino oscillation probabilities. In the context of this dissertation, matter effects are not significant, as shown in Chapter 2.

Most of the three-flavor neutrino oscillation parameters have been precisely measured and consistent observations are made using neutrinos from solar, atmospheric, reactor and accelerator beam sources [17, 25-30]. The global fit result from Ref. [31] is shown in Table 1.1. It should be noted that the values used in this dissertation for the $\Delta m_{21}^{2}$ mass squared splitting and 
$\theta_{12}$ mixing angle are taken from a previous global fit by the same authors, discussed in Ref. [32]. There are three remaining unknowns within the threeflavor neutrino oscillation model:

- the value of $\delta_{\mathrm{CP}}$;

- the ordering of the neutrino masses, which remains unknown because the sign of $\Delta m_{31}^{2}$ is not known, with $\Delta m_{31}^{2}>0$ defined as normal ordering (NO) and $\Delta m_{31}^{2}<0$ defined as inverted ordering (IO), as illustrated in the left panel of Fig. 1.2; and

- the octant of $\theta_{23}$, for which it is not known whether $\theta_{23}$ is smaller or larger than $\pi / 4$.

In addition to searching for these unknowns, neutrino oscillation experiments perform precision measurements of the other oscillation parameters. The right panel of Fig. 1.2 shows the neutrino oscillation probabilities as a function of $L / E$ in the context of the MINOS experiment, with a neutrino energy range between 0 and $40 \mathrm{GeV}$ and baselines of $1 \mathrm{~km}$ (denoted as Near) or $735 \mathrm{~km}$ (denoted as Far). The minimum in the muon neutrino disappearance probability $P\left(v_{\mu} \rightarrow v_{\mu}\right)$ occurs around $L / E=500 \mathrm{~km} / \mathrm{GeV}$ and coincides with a maximum in the tau neutrino appearance probability $P\left(v_{v} \rightarrow v_{\tau}\right)$, meaning that a muon neutrino has about $75 \%$ chance to oscillate into a tau neutrino at this value of $L / E$. The MINOS experiment was originally designed to perform precision measurements of the atmospheric neutrino oscillation parameters $\Delta m_{32}^{2}$ 
and $\theta_{23}$. Figure 1.3 shows the MINOS three-flavor fit in the $\left(\sin ^{2} \theta_{23}, \Delta m_{32}^{2}\right)$ plane to atmospheric neutrino and accelerator beam neutrino data, using the muon neutrino disappearance and electron neutrino appearance channels for the latter.

\begin{tabular}{|c|c|c|c|}
\hline Parameter & Mass Ordering & Best fit & $1 \sigma$ range \\
\hline \hline$\delta m^{2} / 10^{-5} \mathrm{eV}^{2}$ & NO or IO & 7.37 & $7.21-7.54$ \\
$\sin ^{2} \theta_{12} / 10^{-1}$ & NO or IO & 2.97 & $2.81-3.14$ \\
\hline$\Delta m^{2} / 10^{-3} \mathrm{eV}^{2}$ & NO & 2.525 & $2.495-2.567$ \\
$\Delta m^{2} / 10^{-3} \mathrm{eV}^{2}$ & IO & 2.505 & $2.473-2.539$ \\
\hline $\sin ^{2} \theta_{13} / 10^{-2}$ & NO & 2.15 & $2.08-2.22$ \\
$\sin ^{2} \theta_{13} / 10^{-2}$ & IO & 2.16 & $2.07-2.24$ \\
\hline $\sin ^{2} \theta_{23} / 10^{-1}$ & NO & 4.25 & $4.10-4.46$ \\
$\sin ^{2} \theta_{23} / 10^{-1}$ & IO & 5.89 & $5.67-6.05$ \\
\hline$\delta_{\mathrm{CP}} / \pi$ & NO & 1.38 & $1.18-1.61$ \\
$\delta_{\mathrm{CP}} / \pi$ & IO & 1.31 & $1.12-1.62$ \\
\hline
\end{tabular}

Table 1.1: Global fit result of the standard three-flavor oscillation model taken from Ref. [31]. Note that $\delta m^{2} \equiv \Delta m_{21}^{2}$ and $\Delta m^{2} \equiv m_{3}^{2}-\left(m_{1}^{2}-m_{2}^{2}\right) / 2$, with $+\Delta m^{2}$ for normal mass ordering (NO) and $-\Delta m^{2}$ for inverted mass ordering (IO). 


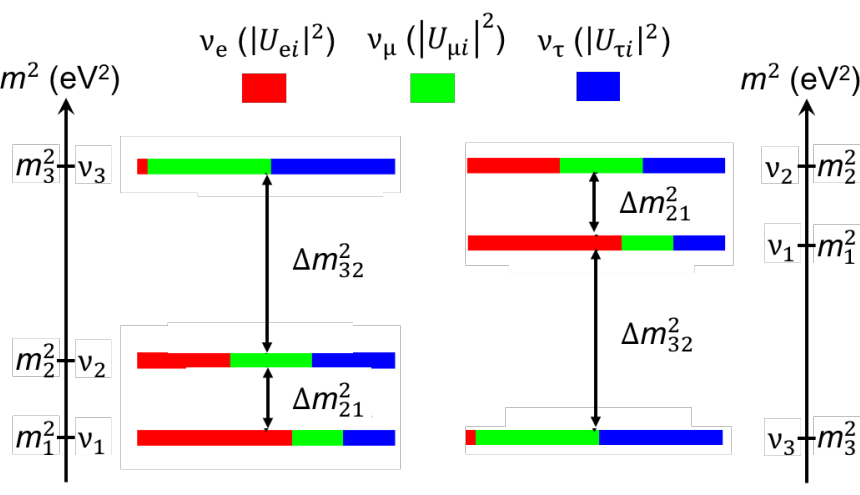

Normal Ordering (NO) Inverted Ordering (IO)

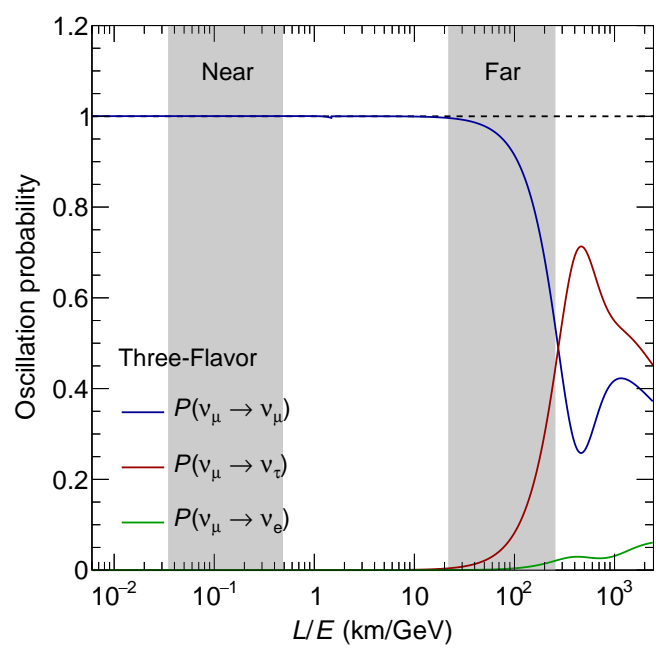

Figure 1.2: The three-flavor neutrino oscillation paradigm. (Left) Illustration of the two possible neutrino mass orderings, normal and inverted ordering. The color coding indicates the fraction $\left|U_{\alpha i}\right|^{2}$ of flavor $v_{\alpha}(\alpha=\mathrm{e}, \mu, \tau)$ contained in mass eigenstate $i$. This figure is a modified version of a figure taken from Ref. [33]. (Right) The muon neutrino disappearance (blue line), tau neutrino appearance (red line), and electron neutrino appearance (green line) probabilities as a function of $L / E$, with $L$ the distance traveled by the neutrino and $E$ the neutrino energy, for the three-flavor case. The oscillation parameters are fixed for all cases [32]: $\Delta m_{32}^{2}=2.37 \times 10^{-3} \mathrm{eV}^{2}, \theta_{23}=0.695, \theta_{13}=0.149, \delta_{\mathrm{CP}}=0, \Delta m_{21}^{2}=7.54 \times 10^{-5} \mathrm{eV}^{2}$, and $\theta_{12}=0.588$. The $L / E$ coverage of the MINOS Near and Far Detectors are represented by the gray bands, which contain $90 \%$ of the MINOS and MINOS+ events between 0 and $40 \mathrm{GeV}$. The MINOS energy resolution is taken into account. 

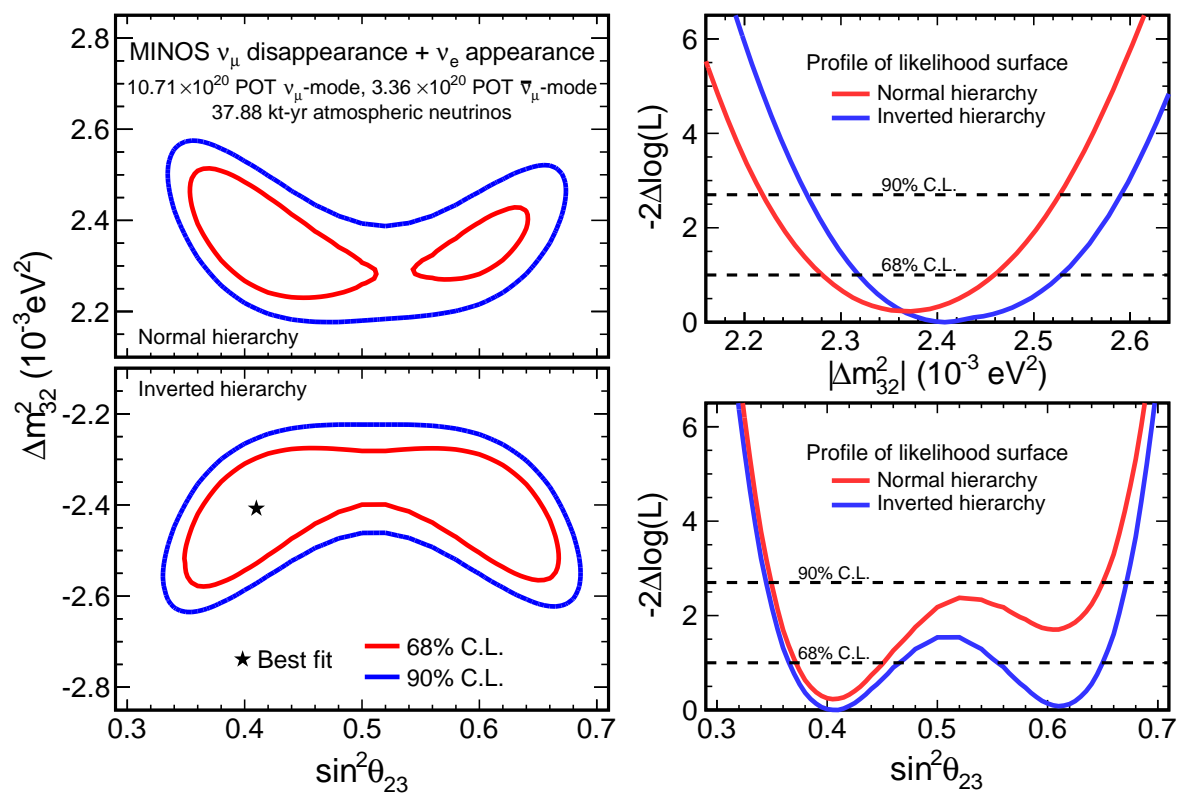

Figure 1.3: A combined three-flavor fit to MINOS muon neutrino disappearance and electron neutrino appearance data and atmospheric neutrino data. (Left) The $68 \%$ and $90 \%$ C.L. limits in the $\left(\sin ^{2} \theta_{23}, \Delta m_{32}^{2}\right)$ plane calculated for normal ordering (top) and inverted ordering (bottom). (Right) The loglikelihood profiles for each mass ordering for $\Delta m_{32}^{2}$ (top) and $\sin ^{2} \theta_{23}$ (bottom). This figure is taken from Ref. [30].

\subsubsection{Two-Flavor Approximation}

It is instructive to look at an effective two-flavor approximation for the muon neutrino disappearance oscillation channel. From Formula (1.8) it follows that

$$
P\left(v_{\mu} \rightarrow v_{\mu}\right)=1-4 \sum_{k>j}\left|U_{\mu k}\right|^{2}\left|U_{\mu j}\right|^{2} \sin ^{2}\left(\frac{1.27 \Delta m_{k j}^{2}\left[\mathrm{eV}^{2}\right] L[\mathrm{~km}]}{E[\mathrm{GeV}]}\right)
$$

where the factor 1.27 in the argument of the sinusoidal arises from unit conversion. Approximating $\sin \theta_{13}=0$ and $\cos \theta_{13}=1$, based on the global fit result 
listed in Table 1.1, the mixing matrix components in Formula (1.10) simplify to

$$
\left|U_{\mu 1}\right|^{2} \approx s_{12}^{2} c_{23}^{2},\left|U_{\mu 2}\right|^{2} \approx c_{12}^{2} c_{23}^{2} \text {, and }\left|U_{\mu 3}\right|^{2} \approx s_{23}^{2}
$$

Table 1.1 shows that the mass squared splitting $\Delta m_{21}^{2}$ is much smaller than $\Delta m_{31}^{2}$ and $\Delta m_{32}^{2}$. Assuming the MINOS Far Detector baseline $L=735 \mathrm{~km}$ and a neutrino energy of $3 \mathrm{GeV}$, the term involving $\Delta m_{21}^{2}$ in Formula (1.10) can be neglected w.r.t. the terms involving $\Delta m_{31}^{2}$ and $\Delta m_{32}^{2}$

$$
\sin ^{2}\left(\frac{1.27 \Delta m_{21}^{2} L}{E}\right) \approx \sin ^{2}\left(\frac{1.27 \times 7 \times 10^{-5} \times 735}{3}\right) \approx \sin ^{2}(0.02) \approx 0 .
$$

Given $\Delta m_{31}^{2} \gg \Delta m_{21}^{2}$ and $\Delta m_{32}^{2}=\Delta m_{31}^{2}-\Delta m_{21}^{2}$, an effective mass squared splitting $\Delta m_{\text {atm }}^{2}=\Delta m_{32}^{2} \approx \Delta m_{31}^{2}$ can be defined to join the remaining two terms in Formula (1.10)

$$
\begin{aligned}
P\left(v_{\mu} \rightarrow v_{\mu}\right) & \approx 1-4 s_{23}^{2} c_{23}^{2}\left(s_{12}^{2}+c_{12}^{2}\right) \sin ^{2}\left(\frac{1.27 \Delta m_{\mathrm{atm}}^{2} L}{E}\right) \\
& =1-\sin ^{2}\left(2 \theta_{23}\right) \sin ^{2}\left(\frac{1.27 \Delta m_{\mathrm{atm}}^{2} L}{E}\right) .
\end{aligned}
$$

Formula (1.13) provides a two-flavor approximation of the muon neutrino disappearance channel shown in the right panel of Fig. 1.2. Only a small component of the muon neutrinos oscillate into electron neutrinos, such that this oscillation channel can be approximated by a two-flavor oscillation between muon and tau neutrinos, where the $L / E$ value of the disappearance minimum is driven by the atmospheric mass squared splitting $\Delta m_{\mathrm{atm}}^{2}$ and the magnitude of the disappearance minimum is driven by the mixing angle $\theta_{23}$. 


\subsection{Beyond the Three-Flavor Paradigm}

Even though most of the experimental neutrino oscillation data are consistent with the three-flavor paradigm described in the previous section, some experimental results are in tension with this picture:

- the observation of anomalous appearance of electron antineutrinos in short-baseline accelerator muon antineutrino beams by the LSND [34] and MiniBooNE [35] experiments;

- a depletion of electron neutrino rates w.r.t. the predicted rates from radioactive calibration sources in gallium experiments GALLEX and SAGE [36]; and

- electron antineutrino rate deficits observed in reactor neutrino experiments upon comparison with recent reactor flux calculations [37].

It should be noted that the reactor anomaly has been weakened by recent reactor fuel cycle measurements by the Daya Bay experiment [38] and by observations of spectral distortions that are not predicted by flux calculations [39].

These data could be accommodated with additional neutrino states with masses at the eV scale, as illustrated in Fig. 1.4 for the short-baseline electron antineutrino appearance anomalies in LSND and MiniBooNE. Because LEP measurements constrain the number of light active neutrinos to three, as discussed in Section 1.1, such additional neutrino states would not interact with SM particles and are called sterile neutrinos. 

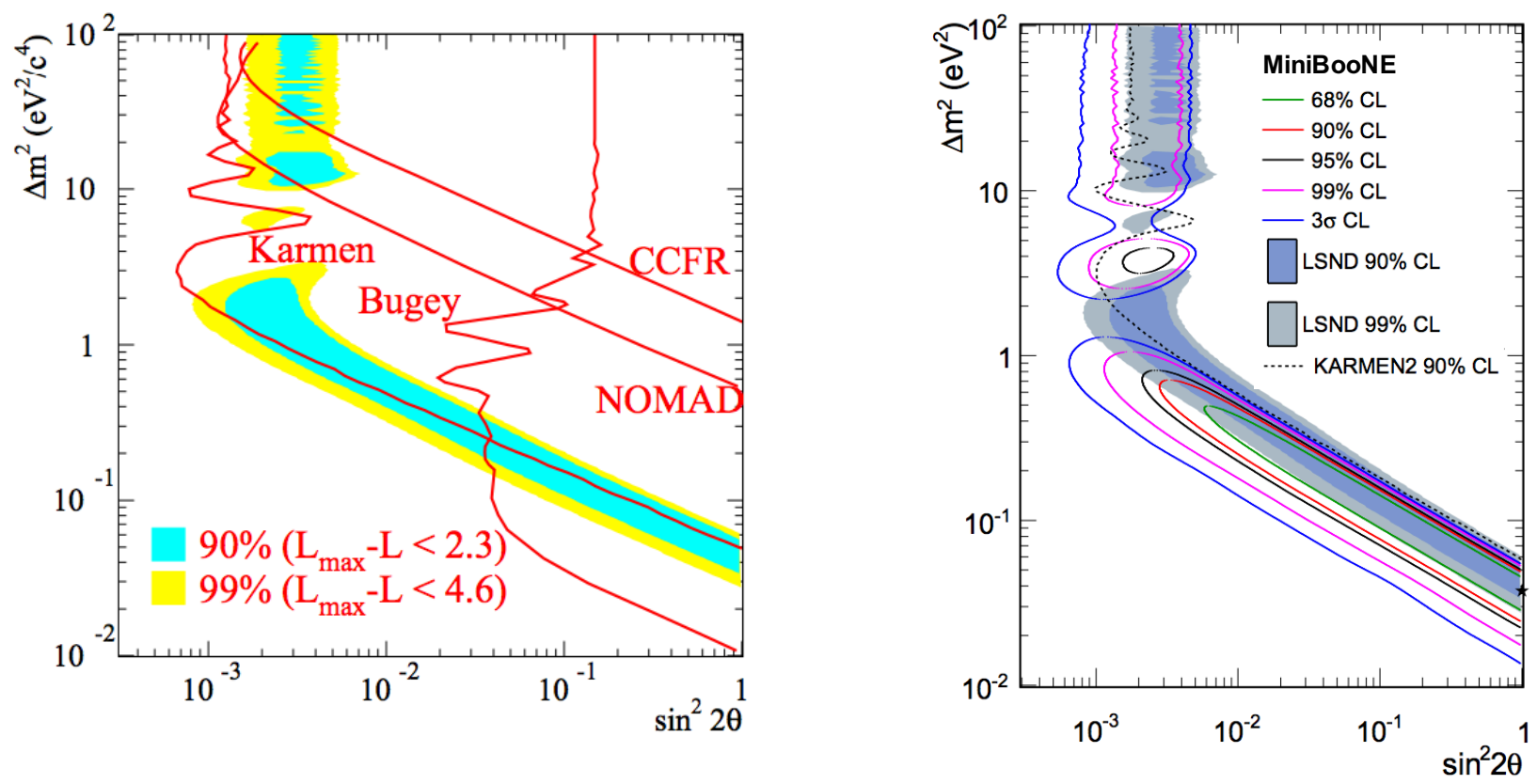

Figure 1.4: The short-baseline anomalies in LSND and MiniBooNE. (Left) A two-flavor fit in the $\left(\sin ^{2}(2 \theta), \Delta m^{2}\right)$ plane for the entire LSND data sample, with $20<E_{\mathrm{e}}<200 \mathrm{MeV}$. The fit includes primary $\bar{v}_{\mu} \rightarrow \bar{v}_{\mathrm{e}}$ oscillations and secondary $\nu_{\mu} \rightarrow v_{\mathrm{e}}$ oscillations, as well as all known neutrino backgrounds. The allowed 90\% C.L. and 99\% C.L. regions are shown in blue and yellow, respectively. Other curves are $90 \%$ C.L. limits from the Bugey reactor experiment [40], the CCFR experiment at Fermilab [41], the NOMAD experiment at CERN [42], and the KARMEN experiment at ISIS [43]. This figure is taken from Ref. [34]. (Right) The MiniBooNE allowed regions in the $\left(\sin ^{2}(2 \theta), \Delta m^{2}\right)$ plane in a combined neutrino and antineutrino mode for events with $200<E_{\nu}^{\mathrm{QE}}<3000 \mathrm{MeV}$ within a two-neutrino $v_{\mu} \rightarrow v_{\mathrm{e}}$ and $\bar{v}_{\mu} \rightarrow \bar{v}_{\mathrm{e}}$ oscillation model. Also shown is the $\bar{v}_{\mu} \rightarrow \bar{v}_{\mathrm{e}}$ limit from the KARMEN experiment [44]. The shaded areas show the $90 \%$ and $99 \%$ C.L. LSND $\bar{v}_{\mu} \rightarrow \bar{v}_{\mathrm{e}}$ allowed regions. The black star shows the best fit point. This figure is taken from Ref. [35]. The legend was modified for clarity. 
A simple model of neutrino flavor mixing that involves a sterile neutrino is the $3+1$ model, in which a fourth mass eigenstate $v_{4}$ and flavor eigenstate $v_{\mathrm{s}}$ are added to the three-flavor model. This is achieved by extending the PMNS matrix to a $4 \times 4$ matrix, which when parametrized in a similar way as in Formula (1.9) introduces three additional mixing angles, $\theta_{14}, \theta_{24}$, and $\theta_{34}$, and two additional CP-violating phases, $\delta_{14}$ and $\delta_{24}$. A third independent mass squared splitting can be defined as $\Delta m_{41}^{2}=m_{4}^{2}-m_{1}^{2}$.

Figure 1.5 shows that the effect of a sterile neutrino in the $3+1$ model on the muon neutrino oscillation probabilities strongly depends on the values of $\Delta m_{41}^{2}$ and $\theta_{24}$. A more detailed discussion of the $3+1$ model is outlined in Ref. $[45,46]$. The left panel of Fig. 1.6 shows the constraints on the $3+1$ model reported by the MINOS collaboration [47] using a Far-over-Near analysis method that is also employed in Chapter 5 of this dissertation. The combination of the MINOS and Daya Bay/Bugey-3 sterile searches [48], using the muon neutrino disappearance and electron antineutrino disappearance channels, respectively, allows a comparison with the LSND and MiniBooNE results, as shown in the right panel of Fig. 1.6. As part of this dissertation, a study of fluctuated sensitivities is performed in the context of the $3+1$ model and using the new two-detector analysis method, and is presented in Appendix B. 

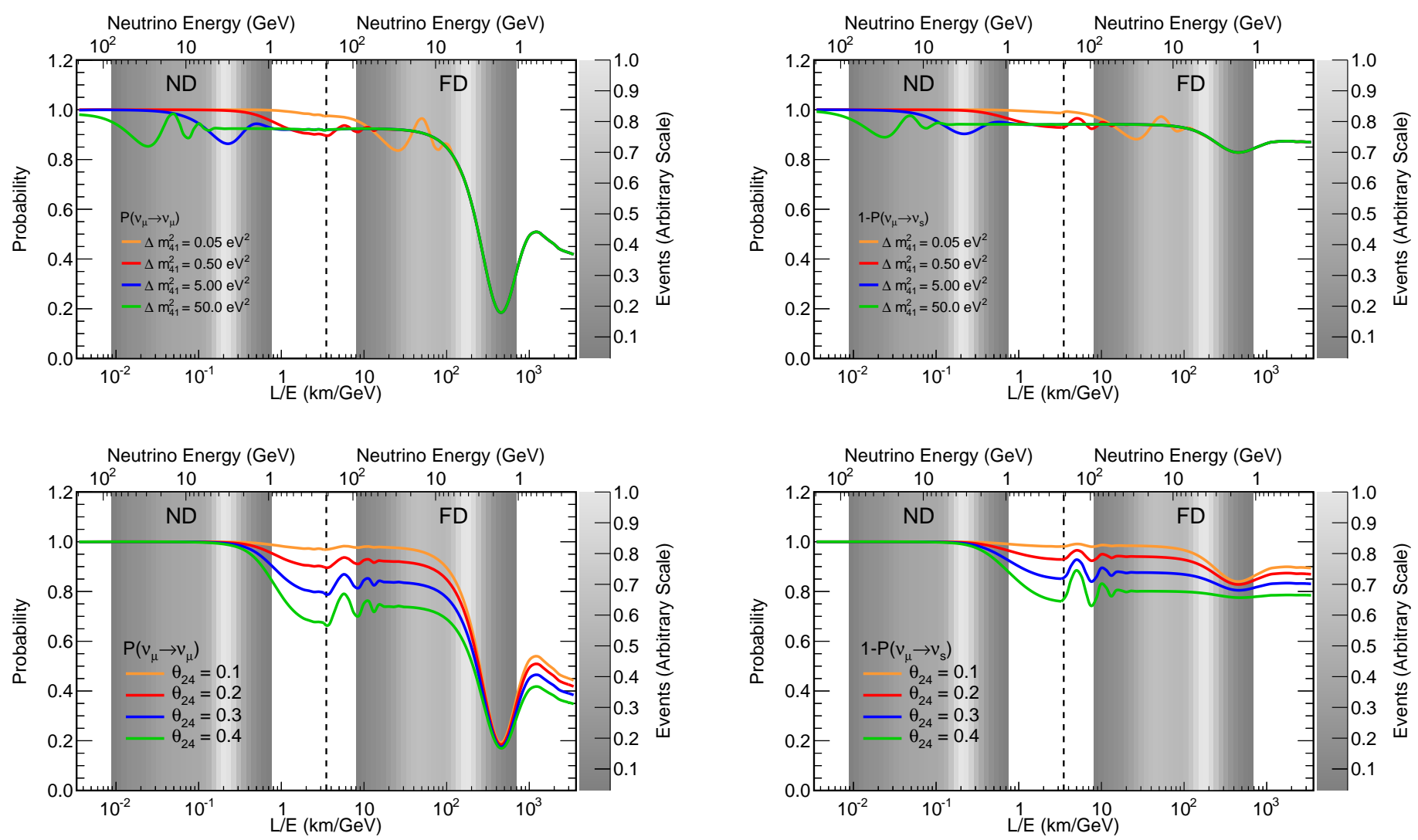

Figure 1.5: The effect of $\Delta m_{41}^{2}$ (top row) and $\theta_{24}$ (bottom row) on the muon disappearance (left column) and sterile appearance (right column) probabilities as a function of $L / E$ in the sterile $3+1$ model. Unless indicated otherwise, the parameter values are given by: $\Delta m_{21}^{2}=7.54 \times 10^{-5} \mathrm{eV}^{2}, \Delta m_{21}^{2}=2.37 \times 10^{-3} \mathrm{eV}^{2}$, $\Delta m_{41}^{2}=0.5 \mathrm{eV}^{2}, \theta_{12}=0.554, \theta_{13}=0.149, \theta_{23}=0.695, \theta_{14}=0, \theta_{24}=0.2, \theta_{34}=0.5, \delta_{13}=0, \delta_{14}=0$, and $\delta_{24}=0$. These figures are taken from Ref. [45]. 

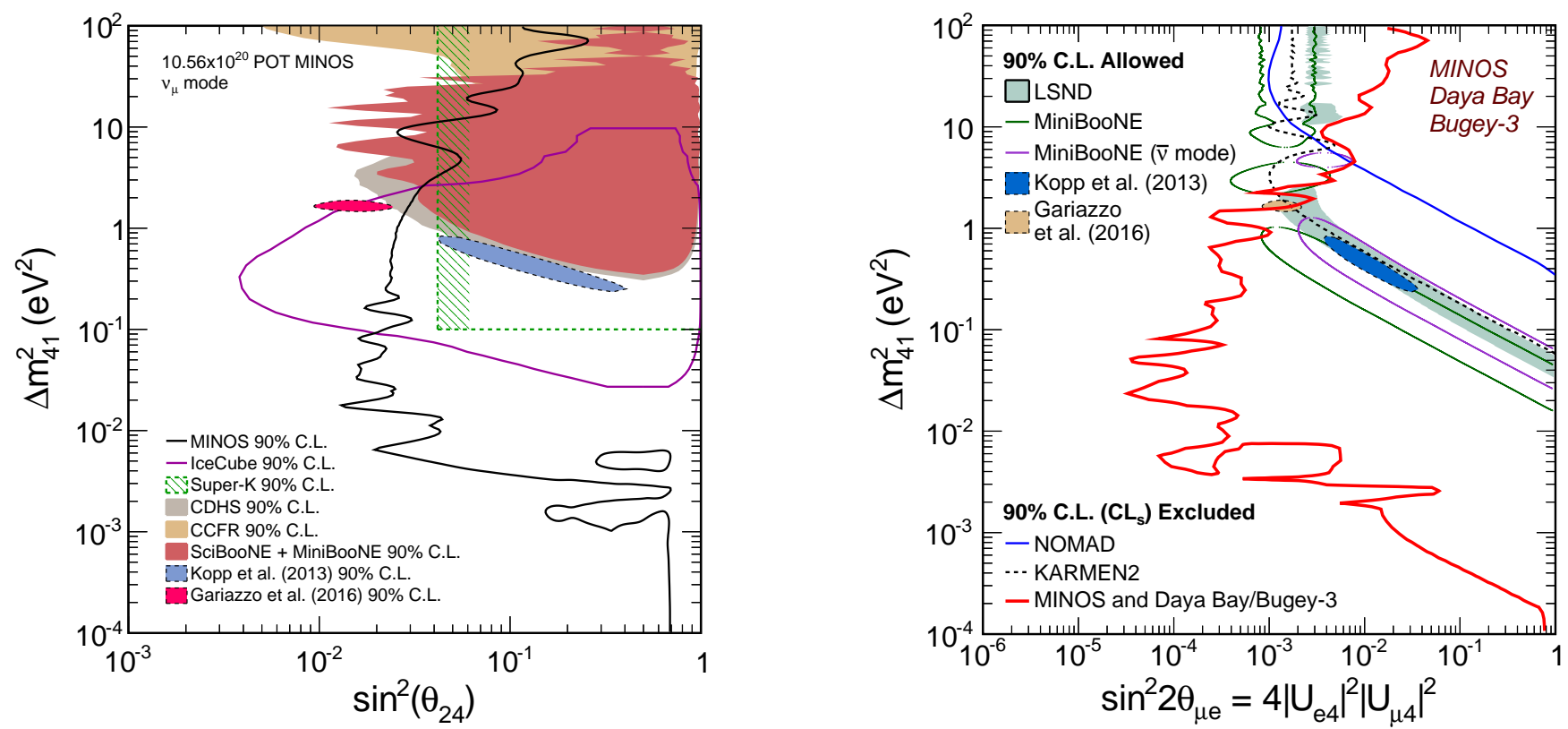

Figure 1.6: Constraining the 3+1 sterile neutrino model with MINOS. (Left) The Feldman-Cousins $90 \%$ C.L. data coverage for MINOS using $v_{\mu}$ disappearance and searching for a deficit in neutral current events. These contours are compared to other experiments. Note the axis is $\sin ^{2}\left(\theta_{24}\right)$. All experiments except Super-K and MINOS reported results in terms of $\sin ^{2}\left(2 \theta_{24}\right)$, so their data coverages were made symmetric when converting to $\sin ^{2}\left(\theta_{24}\right)$. Also shown are the $90 \%$ C.L. allowed regions of two global fits. The Kopp et al. 2013 fit included $v_{\mathrm{e}}$ appearance experiments, and the Gariazzo et al. 2016 fit included all shortbaseline experiments with the exception of MiniBooNE data below $475 \mathrm{MeV}$. To compare these global fits to disappearance data, $\sin ^{2} 2 \theta_{\mu \mathrm{e}}$ is converted to $\sin ^{2} \theta_{24}$ by assuming $\theta_{14}=0.15$, the best fit value from the Kopp et al. global fit to appearance data. This figure is taken from Ref. [47]. (Right) The MINOS and Daya Bay/Bugey-3 combined $90 \% \mathrm{CL}_{\mathrm{S}}$ data limit on $\sin ^{2} 2 \theta_{\mu \mathrm{e}}$ compared to the LSND and MiniBooNE $90 \%$ C.L. allowed regions. Regions of parameter space to the right of the red contour are excluded. The regions excluded at $90 \%$ C.L. by KARMEN2 and NOMAD are also shown. The excursion to small mixing in the exclusion contour at about $\Delta m_{41}^{2} \sim 5 \times 10^{-3} \mathrm{eV}^{2}$ is due to the degenerate island in the MINOS exclusion limit. This figure is taken from Ref. [48]. 
Independently of whether these anomalies are true or not, precision measurements with neutrino oscillation experiments provide ways to search for physics beyond the SM. This dissertation presents a search for physics beyond the three-flavor paradigm using the Large Extra Dimensions model, where sterile neutrinos arise as Kaluza-Klein states in an extra spatial dimension. This model is discussed in detail in Chapter 2. Chapter 5 and Chapter 6 present two different analysis strategies used to search for large extra dimensions employing the MINOS and MINOS+ accelerator beam neutrino data. 


\title{
Chapter 2
}

\section{Large Extra Dimensions}

\begin{abstract}
Although most data from solar, atmospheric, reactor, and accelerator beam neutrino oscillation experiments are consistent with the existence of the three active neutrino flavors, $v_{\mathrm{e}}, \boldsymbol{v}_{\mu}$, and $\boldsymbol{v}_{\tau}$, there have been experimental hints of the existence of additional neutrino mass states with masses at the eV scale, as discussed in Section 1.3. With increasing experimental precision, alternate neutrino oscillation scenarios that include additional neutrino mass states can be tested. This chapter introduces one such scenario, the Large Extra Dimensions (LED) model. This model is tested in this dissertation employing the rich and unique data collected by the MINOS and MINOS+ experiments.
\end{abstract}

\subsection{Large Extra Dimensions Model}

Sub-millimeter sized flat large extra dimensions were originally introduced in the ADD model in Ref. [49] to explain the large gap between the electroweak energy scale, $m_{\mathrm{EW}} \sim 10^{3} \mathrm{GeV}$, and the Planck scale, $M_{\mathrm{Pl}} \sim 10^{19} \mathrm{GeV}$. Other models assume warped extra dimensions to explain the high Planck scale $[50,51]$. In the ADD model, $M_{\mathrm{Pl}}$ attains its high value due to a volu- 
metric scaling of a more fundamental scale, $\bar{M}_{\mathrm{Pl}}$, which is assumed to be of the same order of magnitude as $m_{\mathrm{EW}}$,

$$
M_{\mathrm{Pl}}^{2}=\bar{M}_{\mathrm{Pl}}^{d+2} V_{d}
$$

where $d$ is the number of extra dimensions and $V_{d}$ the corresponding volume. In this framework, however, the lack of a higher fundamental scale disqualifies the see-saw mechanism [52-55] as an explanation of the small neutrino masses. To resolve this, the existence of sterile neutrinos, arising as Kaluza-Klein (KK) states in the extra dimensions, is suggested in Ref. [56, 57], leading to small Dirac neutrino masses [57]

$$
m_{v}=\kappa v \frac{\bar{M}_{\mathrm{Pl}}}{M_{\mathrm{Pl}}}
$$

where $\kappa$ is a Yukawa coupling coefficient and $v$ the Higgs vacuum expectation value.

Adopting the LED model of Ref. [58-64], all the SM fields, including the three left-handed (active) neutrinos and the Higgs doublet, live on a fourdimensional brane, $3+1$ spacetime. Three SM singlet fermion fields, one for each neutrino flavor, live in a higher-dimensional bulk, $3+1+d$ spacetime, with at least two compactified extra dimensions $(d \geq 2)$. To simplify matters, one of the extra dimensions can be compactified on a circle with radius $R$ much larger than the size of the other dimensions, effectively making this a five-dimensional problem. The compactness of the extra dimension allows a decomposition of each bulk fermion in Fourier modes. From the couplings to gauge bosons, the 
zero modes can be identified as the active neutrinos, while the other modes are sterile neutrinos. All these states are collectively referred to as the KK towers. The Yukawa couplings between the bulk fermions and the SM Higgs field and active neutrinos lead to mixing between the SM and KK neutrinos, which alters the three-flavor neutrino oscillation probabilities. Hence, neutrino oscillation measurements can constrain the size of large extra dimensions.

As discussed in Ref. [64], the oscillation amplitude among active neutrino states can be written as

$$
\mathcal{A}\left(v_{\alpha} \rightarrow v_{\beta}\right)=\sum_{i, j, k=1}^{3} \sum_{n=0}^{+\infty} U_{\alpha i} U_{\beta k}^{*} W_{i j}^{(0 n) *} W_{k j}^{(0 n)} \exp \left[i\left(\frac{\lambda_{j}^{(n)}}{R}\right)^{2}\left(\frac{L}{2 E}\right)\right]
$$

where $E$ is the neutrino energy and $L$ the baseline. The eigenvalues $\lambda_{j}^{(n)}$ of the Hamiltonian depend on the extra dimension size $R$ and the active neutrino masses $m_{1}, m_{2}$, and $m_{3}$. The mass $m_{\mathrm{KK}, j}^{(n)}$ of KK mode $n$ in tower $j$ is given by $\lambda_{j}^{(n)} / R$. The matrices $U$ and $W$ are the mixing matrices for the active and KK neutrino modes, respectively. The $(0 n)$ indices refer to the mixing between the zero or active mode and the KK tower. In vacuum, KK modes connected to different generations decouple such that $W_{i j}^{(0 n)}=W_{i}^{(0 n)}$. Squaring the amplitude gives the oscillation probability $P\left(v_{\alpha} \rightarrow v_{\beta}\right)$. Compared to the three-flavor case, this model requires two extra parameters, $R$ and $m_{0}$, where the latter is defined as the lightest active neutrino mass. For normal mass ordering this implies that $m_{3}>m_{2}>m_{1} \equiv m_{0}$, while for inverted mass ordering $m_{2}>m_{1}>m_{3} \equiv m_{0}$. Sections 2.3 and 2.4 explore the LED model in more detail. 


\subsection{Status of Searches for Extra Dimensions}

It was shown in the previous section that neutrino oscillations provide a novel way to search for large extra dimensions. This section summarizes the status of searches for extra dimensions through neutrino oscillations and other channels.

\subsubsection{Neutrino Oscillation Searches}

Various sensitivities for neutrino oscillation experiments have been reported. Reference [63] discusses the constraints on the LED model introduced in the previous section, based on atmospheric, reactor, and accelerator beam neutrino oscillation experiments. The extra dimension size $R$ is derived to be less than $0.82 \mu \mathrm{m}$ at $90 \%$ C.L. Reference [64] discusses the sensitivities of CHOOZ, KamLAND, and MINOS for this model, with a combined sensitivity of $R<0.75(0.49) \mu \mathrm{m}$ at $90 \%$ C.L. obtained for normal (inverted) mass ordering and in the limit of vanishing $m_{0}$ values. The top left panel of Fig. 2.1 shows the MINOS sensitivity to the LED model in the $\left(R, m_{0}\right)$ plane as calculated in Ref. [64]. This result was successfully reproduced in the initial stages of the MINOS LED analysis outlined in Ref. [45]. In Ref. [65], an exclusion

of $R \gtrsim 0.40 \mu \mathrm{m}$ at $95 \%$ C.L. is derived based on the zenith distribution of atmospheric neutrino events collected by the IceCube experiment, as shown in the bottom left panel of Fig. 2.1. The same authors show that it is possible to translate the LED model with $n$ KK modes in each tower to a $3+3 n$ sterile neutrino model, consisting of the three active neutrinos and $3 n$ sterile 
neutrinos. This is illustrated for $n=3$ in the top right panel of Fig. 2.1. This relation between the LED model and sterile neutrino models is also discussed in Ref. [66] in the context of the future DUNE long-baseline neutrino oscillation experiment.

It was found in Ref. [63] and explored in more detail in Ref. [67] that the gallium and reactor antineutrino anomalies could be accomodated by possible oscillations of electron neutrinos and antineutrinos into sterile KK neutrinos in an LED model with $R \lesssim 0.6 \mu \mathrm{m}$. However, the electron antineutrino excess observed in LSND and MiniBooNE cannot be explained by the LED model presented above. It is shown in Ref. [68] that in an extended LED model, LED+, with the assumed existence of three additional bulk masses, sterile neutrinos propagating in large extra dimensions could induce electron antineutrino appearance and as such accomodate the LSND and MiniBooNE anomalies. The bottom left panel of Fig. 2.1 shows the sensitivity to the LED+ model for MINOS, reactor short baseline experiments, DUNE, and SBN.

\subsubsection{Other Searches}

Other searches for extra dimensions include tabletop gravitational experiments, where possible modifications to the gravitational potential due to the existence of extra dimensions are searched for. Bounds on the extra di-

mension size $R$ from tabletop gravitational experiments are about 2 orders of magnitude weaker than those from neutrino oscillation experiments [17]. 

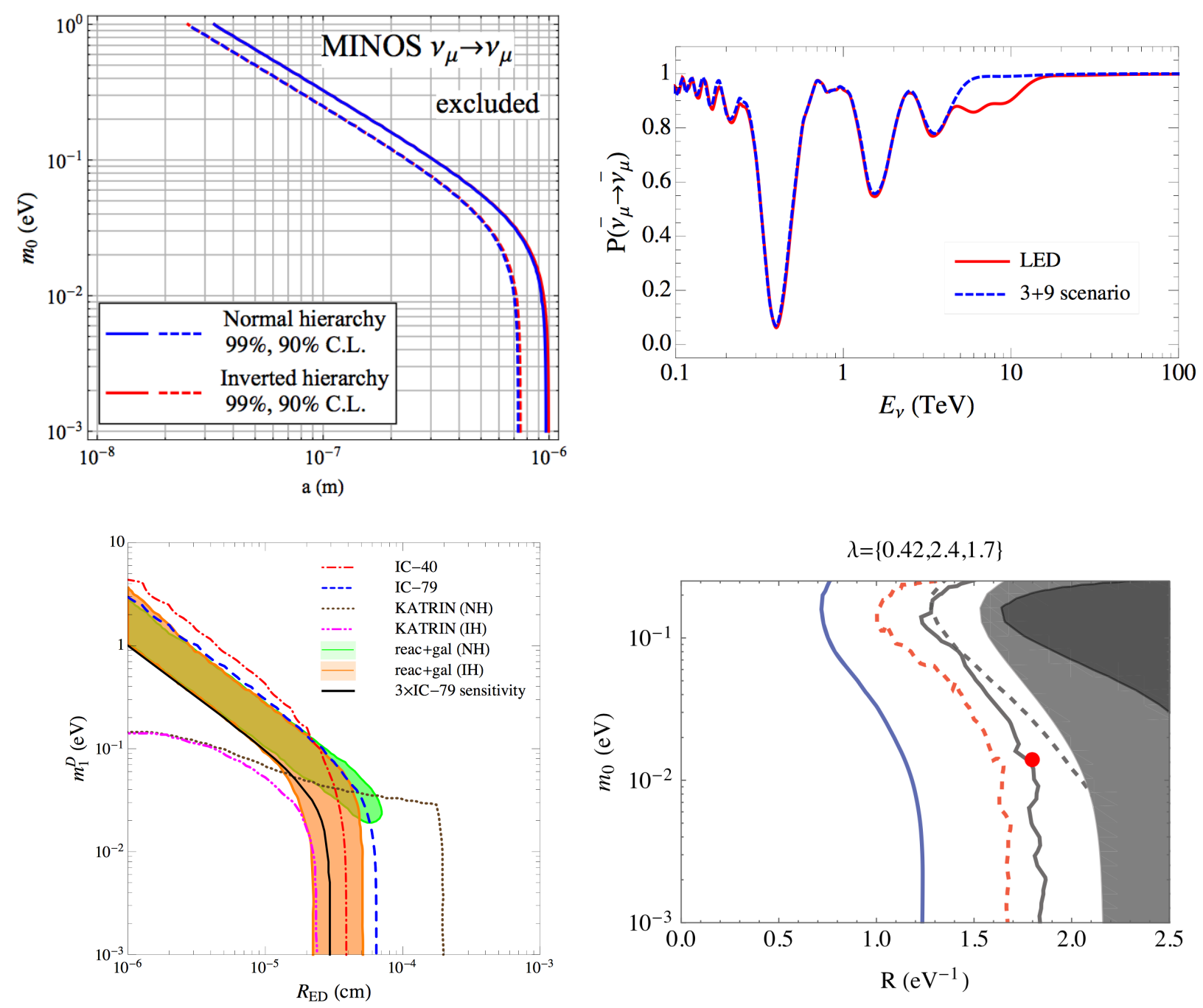

Figure 2.1: Searches for extra dimensions with neutrino oscillations. (Top left) The MINOS sensitivity to the LED model in the $\left(R, m_{0}\right)$ plane as calculated in Ref. [64]. (Bottom left) The sensitivity to the LED model obtained from atmospheric neutrino data collected by the IceCube experiment (red and blue dashed lines) and presented in Ref. [65]. The other confidence regions in this figure are estimated sensitivities. (Top right) The relation between the LED model and the $3+3 n$ sterile neutrino model for $n=3$, where $n$ is the number of KK modes considered in each tower, in the muon antineutrino disappearance channel calculated in Ref. [65]. (Bottom right) The sensitivity to the LED+ model for MINOS (solid gray line), reactor short baseline neutrino oscillation experiments (gray dashed line), DUNE (red dashed line), and SBN (blue line), calculated in Ref. [68] (unit conversion: $1 \mu \mathrm{m}=5 \mathrm{eV}^{-1}$ ). 
Astrophysical and cosmological bounds are often much more constraining than those from neutrino oscillation experiments, but are model dependent [17]. Collider experiments can set limits on the volume rather than the size of the extra dimensions [17].

A global picture provided by Machado, Nunokawa, and Zukanovich Funchal was shown in Ref. [45] and is included here for completeness.

\subsection{Searching for Large Extra Dimensions with MINOS and MINOS+}

The two relevant oscillation channels for the MINOS/MINOS+ LED analyses are the muon neutrino disappearance channel and the sterile neutrino appearance channel. The former is probed by Charged Current (CC) events in the MINOS Near Detector (ND) and Far Detector (FD) and from Formula (2.3) it follows that it has an oscillation amplitude in vacuum equal to

$$
P\left(v_{\mu} \rightarrow v_{\mu}\right)=\left|\sum_{j=1}^{3} \sum_{n=0}^{+\infty} U_{\mu j} U_{\mu j}^{*}\left(W_{j}^{(0 n)}\right)^{2} \exp \left[i\left(\frac{\lambda_{j}^{(n)}}{R}\right)^{2}\left(\frac{L}{2 E}\right)\right]\right|^{2} .
$$

The latter channel is probed by a depletion of Neutral Current (NC) events in the MINOS detectors and has an oscillation amplitude in vacuum [63] equal to

$$
P\left(v_{\mu} \rightarrow v_{\mathrm{s}}\right)=\sum_{j=1}^{3} \sum_{k=1}^{+\infty}\left|U_{\mu j} \sum_{n=1}^{+\infty} W_{j}^{(0 n)} W_{j}^{(k n)} \exp \left[i\left(\frac{\lambda_{j}^{(n)}}{R}\right)^{2}\left(\frac{L}{2 E}\right)\right]\right|^{2} .
$$




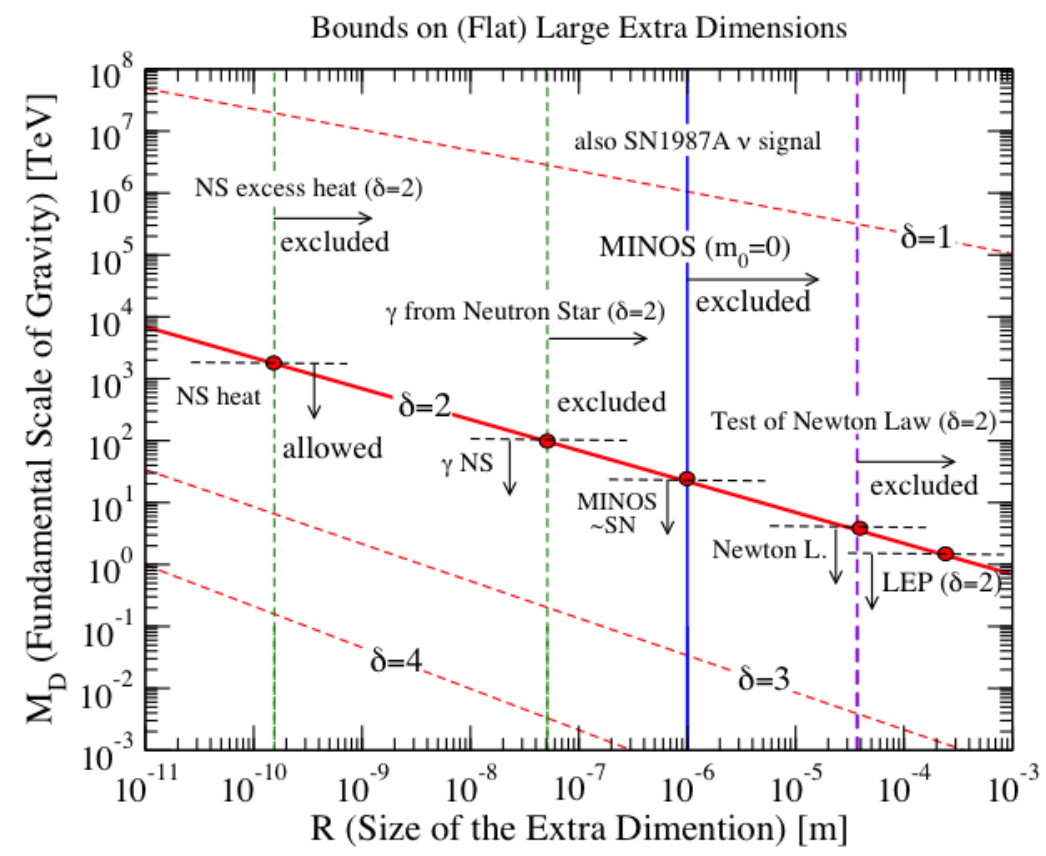

Refs. Giudice and Wells, PDG2010, JPG37, 075021 (2010), p1354 Hannestad and Raffelt, PRD67, 125008 (2003) [Erratum, PRD69, 029901 (2004)]

$$
\overline{\mathrm{M}}_{\mathrm{Pl}}{ }^{2}=\overline{\mathrm{M}}_{\mathrm{D}}{ }^{2+\delta}(2 \pi \mathrm{R})^{\delta}, \mathrm{M}_{\mathrm{D}}=(2 \pi)^{\delta /(2+\delta)} \overline{\mathrm{M}}_{\mathrm{D},} \overline{\mathrm{M}}_{\mathrm{Pl}}=\mathrm{M}_{\mathrm{Pl}} /(2 \pi)^{1 / 2}
$$

Figure 2.2: World's search for extra dimensions. The plot is provided by Machado, Nunokawa and Zukanovich Funchal. $M_{D}, \delta$, and $R$ are the fundamental Planck scale in the bulk, the number of extra dimensions, and the extra dimension size, respectively, where the first two are introduced in Formula (2.1) as $\bar{M}_{\mathrm{Pl}}$ and $d$. The left green dashed line $\left(M_{D}>1700 \mathrm{TeV}\right.$ for $\left.\delta=2\right)$ is the limit from requiring that neutron stars are not excessively heated by KK graviton decays into photons [69]. The right green dashed line $\left(M_{D}>100 \mathrm{TeV}\right.$ for $\delta=2$ ) is the cosmological constraint based on the restriction on the amount of relic gravitons in the universe [70]. The solid blue line $\left(M_{D}>27 \mathrm{TeV}\right.$ for $\delta=2$ ) is the limit from supernova SN1987A requiring that the graviton luminosity agrees with certain stellar models [71]. The MINOS limit shown in blue is based on the simulations done by the authors of this plot. The purple dashed line $(R<37 \mu \mathrm{m}$ at $95 \%$ CL for $n=2)$ is the limit from torsion balance experiments [72]. The right-most red circle $\left(M_{D}>1.60 \mathrm{TeV}\right.$ for $\left.\delta=2\right)$ is the limit from a combination of the LEP results on graviton emission $[73,74]$. 
The oscillation probabilities are such that

$$
P\left(v_{\mu} \rightarrow v_{\mu}\right)+P\left(v_{\mu} \rightarrow v_{\mathrm{e}}\right)+P\left(v_{\mu} \rightarrow v_{\tau}\right)+P\left(\nu_{\mu} \rightarrow v_{\mathrm{s}}\right)=1
$$

In the left of Fig. 2.3, the muon neutrino survival probability, $P\left(\nu_{\mu} \rightarrow v_{\mu}\right)$, for the MINOS FD baseline and normal mass ordering, is illustrated for $m_{0}=0 \mathrm{eV}$ and two values of $R$. As stated in Ref. [64], there are three prominent features of LED visible in this figure: a displacement of the oscillation minimum with respect to the three-flavor case, a reduction of the integrated survival probability because of active-to-KK oscillation, and the appearance of modulations on the survival probability because of fast oscillations to the KK states. With increasing energy, the amplitude of the modulations increases while their frequency decreases, making the effects of LED easier to observe away from the oscillation minimum. The values in Fig. 2.3 of $\Delta m_{32}^{2}$ and $\sin ^{2} \theta_{32}$ are taken from the MINOS standard oscillation analysis [30]. The value of $\sin ^{2} \theta_{13}$ is a weighted average of the Daya Bay [75], RENO [76], and Double Chooz [77] results. The values of $\Delta m_{21}^{2}$ and $\sin ^{2} \theta_{12}$ are taken from Ref [32]. We set $\delta_{\mathrm{CP}}=0$ since it has little effect on the oscillation probabilities [45], as is shown below. These values are used throughout this dissertation. Figures 2.4, 2.5, and 2.6 show the muon neutrino disappearance and sterile neutrino appearance probabilities in the ND and FD for three different points in the $\left(R, m_{0}\right)$ plane, with and without accounting for MINOS energy resolution effects $[78,79]$, which are discussed in more detail in Section 3.7. Note that the effects of the LED model in the ND manifest at lower energies, unlike in the FD. 


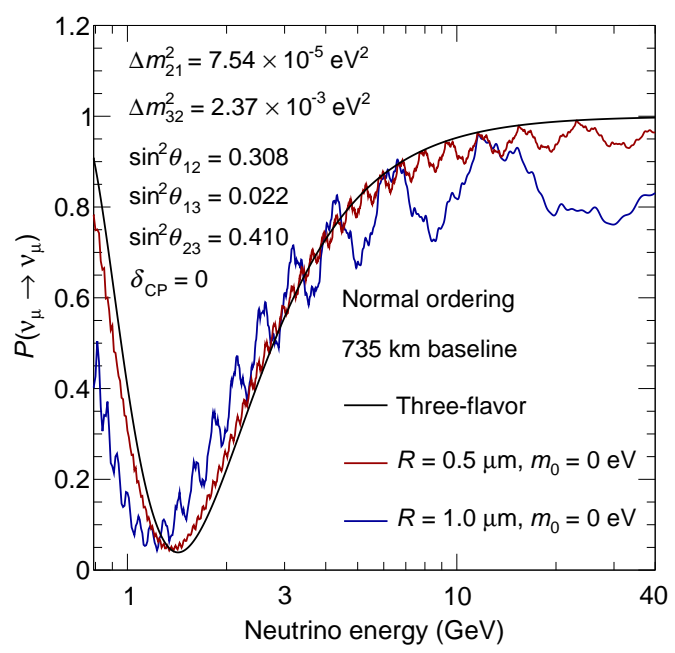

Figure 2.3: The muon neutrino survival probability $P\left(v_{\mu} \rightarrow v_{\mu}\right)$ at the MINOS FD as a function of the true neutrino energy for $m_{0}=0 \mathrm{eV}$ and $R=0.5 \mu \mathrm{m}$ (red line) or $1 \mu \mathrm{m}$ (blue line), and for three-flavor oscillation (black line).

The probabilities shown in Fig. 2.3 and in the rest of this document as well as the probabilities used to calculate the predictions for the LED analysis were obtained using Formulas (2.3)-(2.6), that is in vacuum, assuming normal mass ordering, and taking into account only the first five KK modes, unless mentioned otherwise. Figure 2.7 demonstrates that these are good assumptions which furthermore significantly reduce computing time. Note that the discontinuity in the probabilities around $L / E=1.5 \mathrm{~km} / \mathrm{GeV}$ is an artificial effect due to the difference in energy resolution at low energies for the ND baseline and the energy resolution at high energies for the FD baseline. To simplify matters, the CC energy resolution was used for the sterile appearance probabilities. 

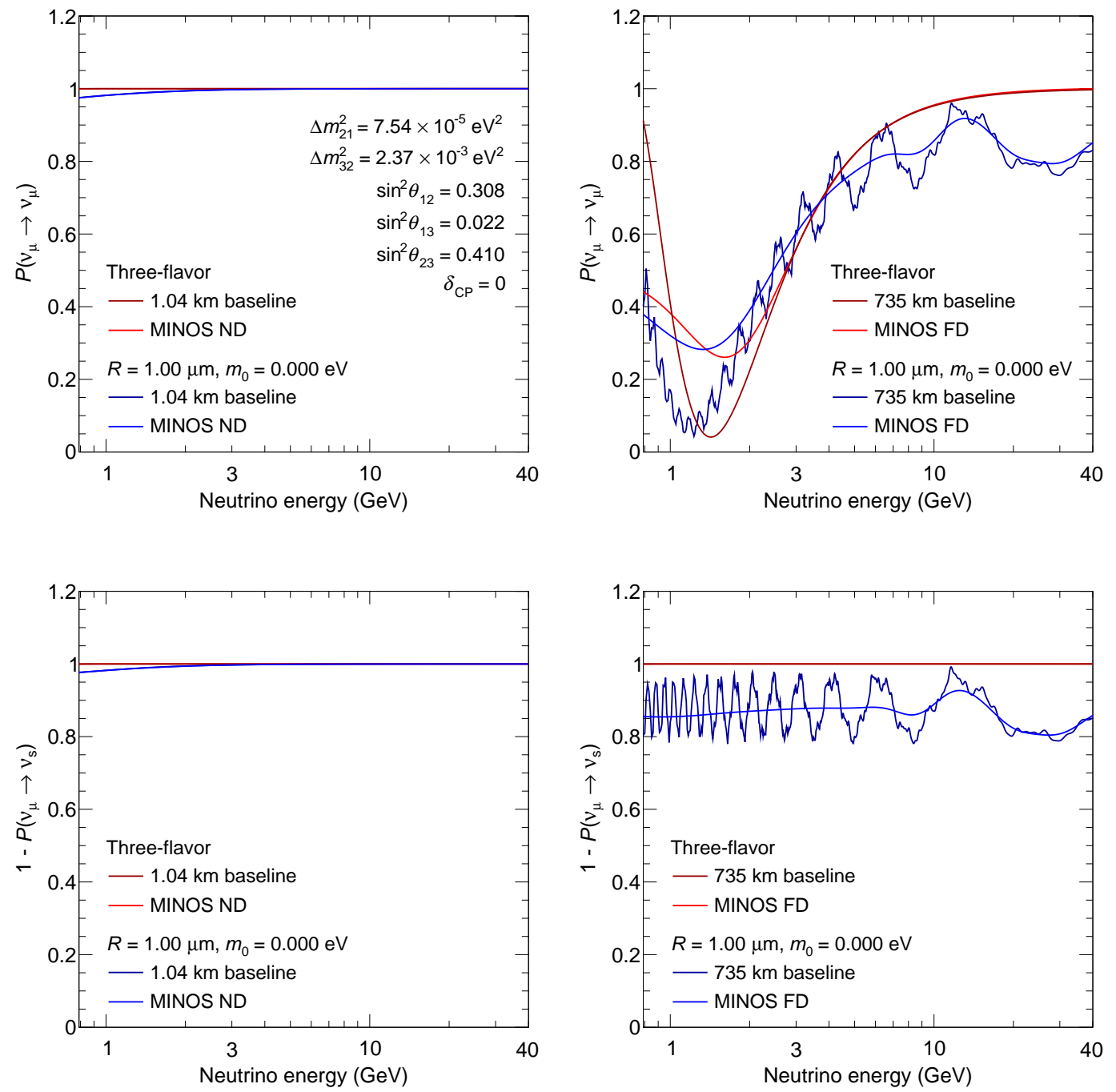

Figure 2.4: The muon neutrino survival probability $P\left(v_{\mu} \rightarrow v_{\mu}\right)$ at the MINOS ND (top left) and FD (top right) and the sterile neutrino appearance probability $P\left(v_{\mu} \rightarrow v_{\mathrm{s}}\right)$ at the ND (bottom left) and FD (bottom right) as a function of the neutrino energy for the three-flavor case (red lines) and for $R=1.00 \mu \mathrm{m}$ and $m_{0}=0.000 \mathrm{eV}$ (blue lines). The effects of the MINOS CC energy resolution are also shown for both baselines. 

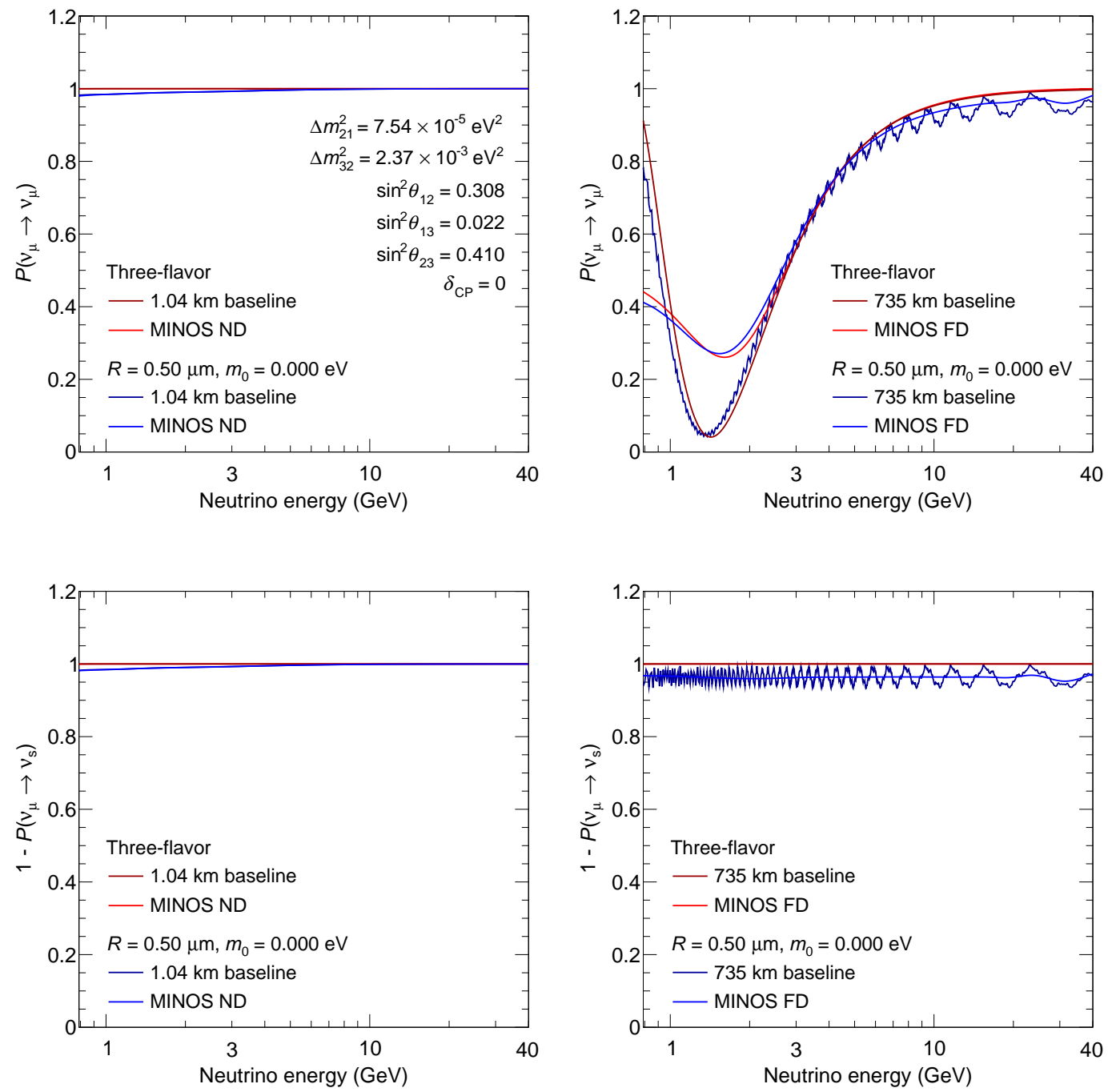

Figure 2.5: The muon neutrino survival probability $P\left(\boldsymbol{v}_{\mu} \rightarrow v_{\mu}\right)$ at the MINOS ND (top left) and FD (top right) and the sterile neutrino appearance probability $P\left(v_{\mu} \rightarrow v_{\mathrm{s}}\right)$ at the ND (bottom left) and FD (bottom right) as a function of the neutrino energy for the three-flavor case (red lines) and for $R=0.50 \mu \mathrm{m}$ and $m_{0}=0.000 \mathrm{eV}$ (blue lines). The effects of the MINOS CC energy resolution are also shown for both baselines. 

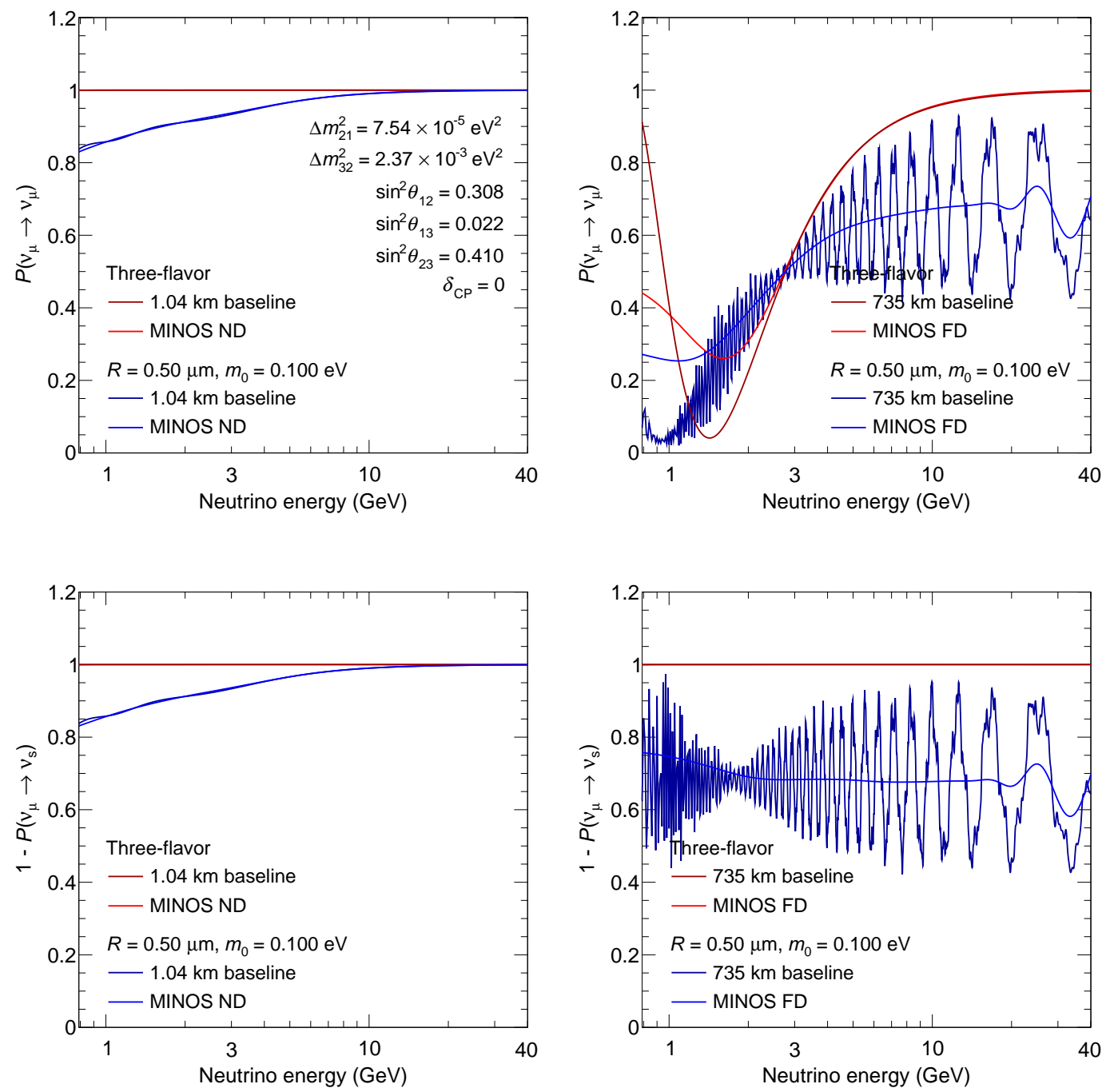

Figure 2.6: The muon neutrino survival probability $P\left(\boldsymbol{v}_{\mu} \rightarrow v_{\mu}\right)$ at the MINOS ND (top left) and FD (top right) and the sterile neutrino appearance probability $P\left(v_{\mu} \rightarrow v_{\mathrm{s}}\right)$ at the ND (bottom left) and FD (bottom right) as a function of the neutrino energy for the three-flavor case (red lines) and for $R=0.50 \mu \mathrm{m}$ and $m_{0}=0.100 \mathrm{eV}$ (blue lines). The effects of the MINOS CC energy resolution are also shown for both baselines. 

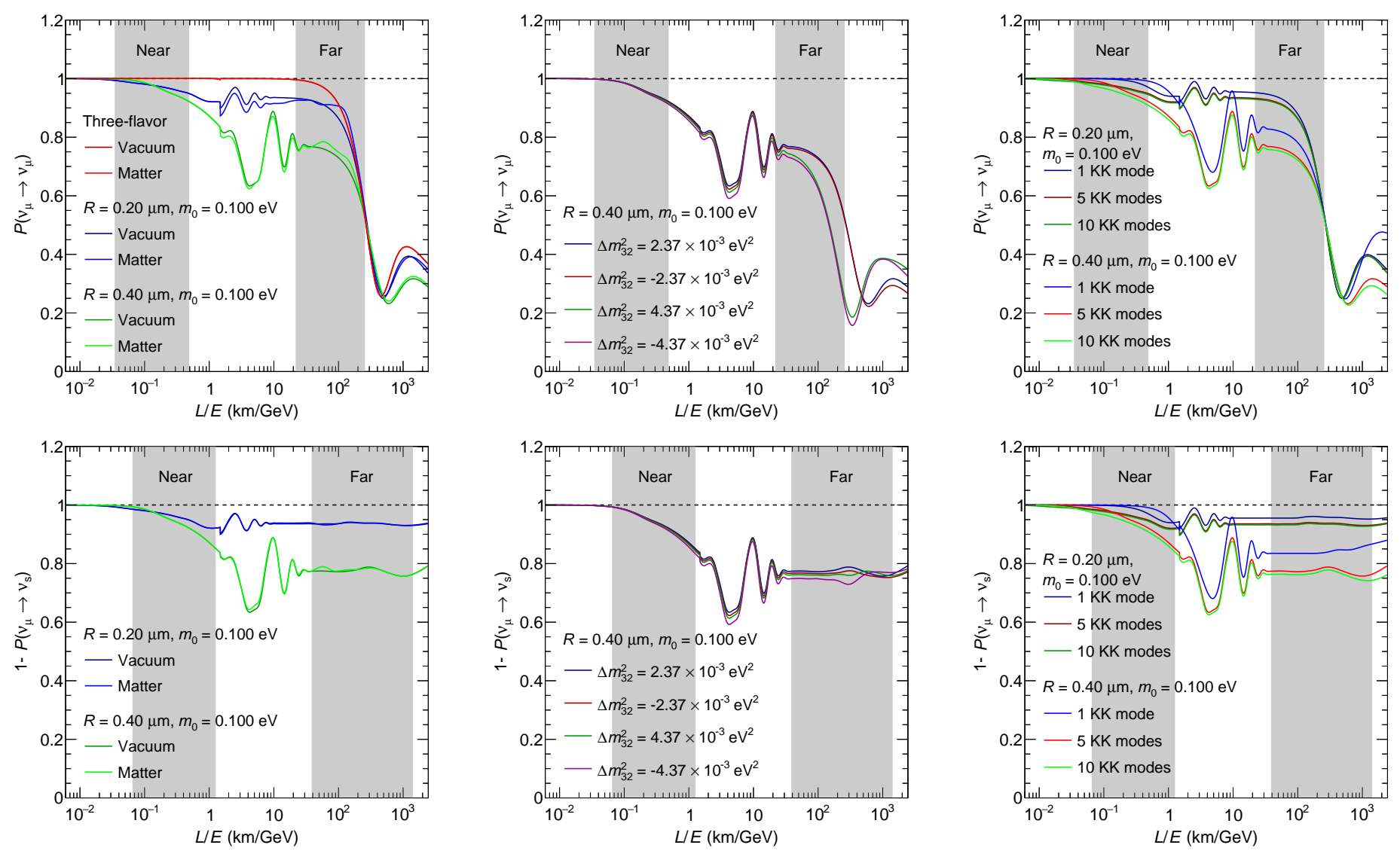

Figure 2.7: The effect of the matter effect (left), the mass ordering (middle), and the number of KK modes (right) on the muon disappearance (top) and sterile appearance (bottom) oscillation probabilities in the MINOS detectors, taking into account energy resolution effects. Parameters not shown in the figures are fixed to their three-flavor values, as shown in Fig. 2.3. 
In the LED model, the magnitude of $\Delta m_{32}^{2}$ and $\theta_{23}$ determine the position and magnitude, respectively, of the oscillation probability minimum in the muon disappearance channel, as a function of $L / E$, and to a lesser extent also in the sterile appearance channel, as shown in Fig. 2.8.

The magnitude of the LED parameters $R$ and $m_{0}$ indicate the presence of oscillations in either or both detectors, as shown in Fig. 2.9. As discussed in more detail in Section 2.4, the magnitude of $m_{0}$ determines the absolute masses of the active neutrinos, while the magnitude of $R$ determines the absolute masses of the sterile neutrinos, where an increase in $R$ and $m_{0}$ decreases the mass squared splittings between the sterile and active neutrinos and within the sterile neutrino towers. At a fixed $m_{0}$, an increase in $R$ increases the overall deficit in neutrino flux at energies away from the three-flavor minimum in the FD and for $R \gtrsim 0.20 \mu \mathrm{m}$ oscillatory behavior can be observed in the FD at higher energies. At a fixed $R$, an increase in $m_{0}$ increases the overall deficit in neutrino flux at energies away from the three-flavor minimum in the FD and causes a significant neutrino flux deficit in the ND for $m_{0} \gtrsim 0.030 \mathrm{eV}$.

The three-flavor parameters $\theta_{13}, \delta_{\mathrm{CP}}, \Delta m_{21}^{2}$, and $\theta_{12}$ have no significant impact on the LED probabilities observed in the MINOS detectors, as shown in Fig. 2.10 and 2.11. 

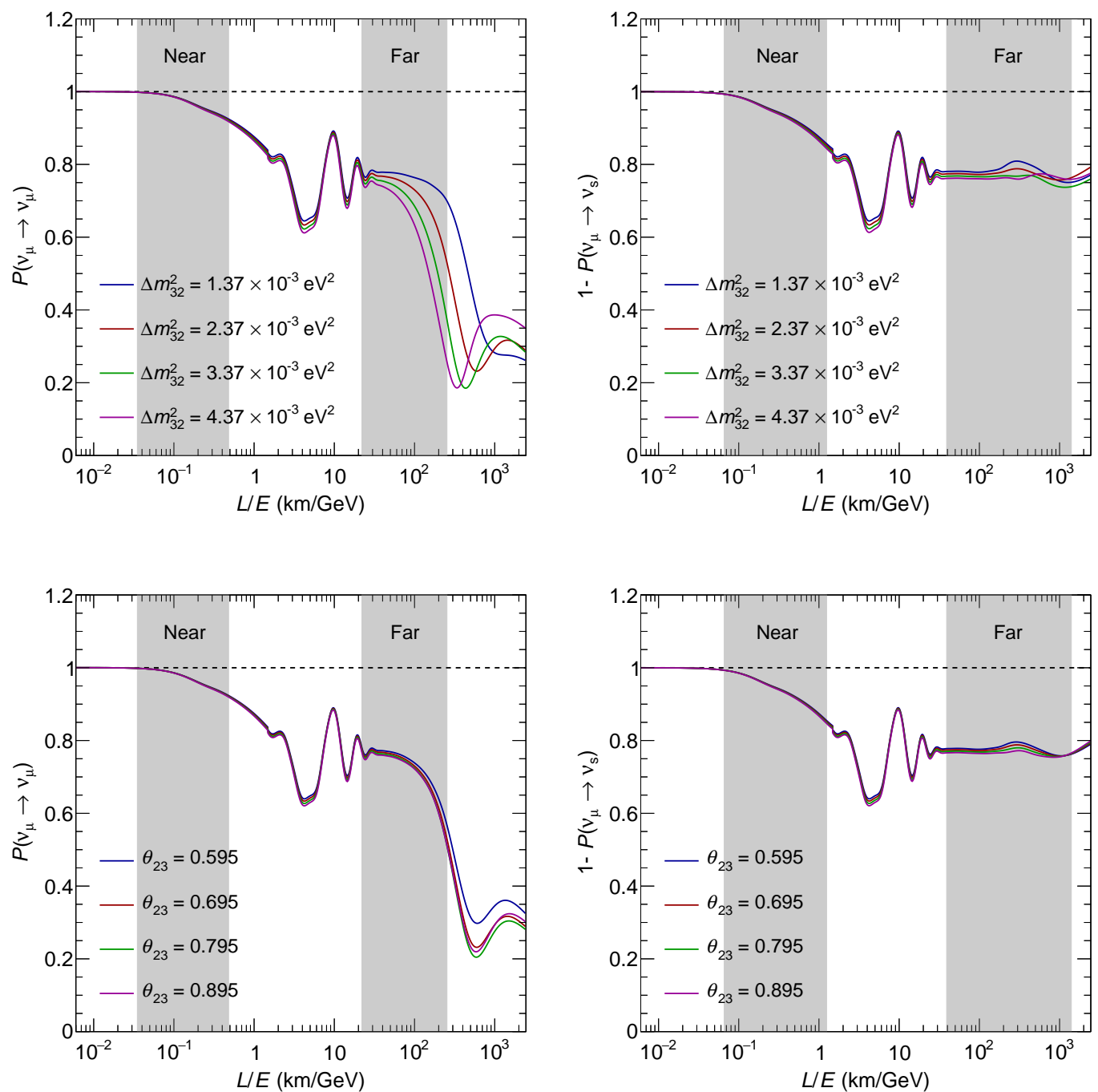

Figure 2.8: Effect of $\Delta m_{32}^{2}$ (top) and $\theta_{23}$ (bottom) on the muon disappearance probability (left) and the sterile appearance probability (right) in the MINOS detectors, taking energy resolution effects into account. For the top plots, $\theta_{23}$ is fixed to 0.695 , while for the bottom plots $\Delta m_{32}^{2}$ is fixed to $2.37 \times 10^{-3} \mathrm{eV}^{2}$. The other oscillation parameters are fixed for all cases: $R=0.40 \mu \mathrm{m}, m_{0}=0.100 \mathrm{eV}, \theta_{13}=0.149, \delta_{\mathrm{CP}}=0, \Delta m_{21}^{2}=7.54 \times 10^{-5} \mathrm{eV}^{2}$, and $\theta_{12}=0.588$. The $L / E$ coverage of the ND and FD are represented by the grey bands, which contain $90 \%$ of the MINOS and MINOS+ events between 0 and $40 \mathrm{GeV}$. 

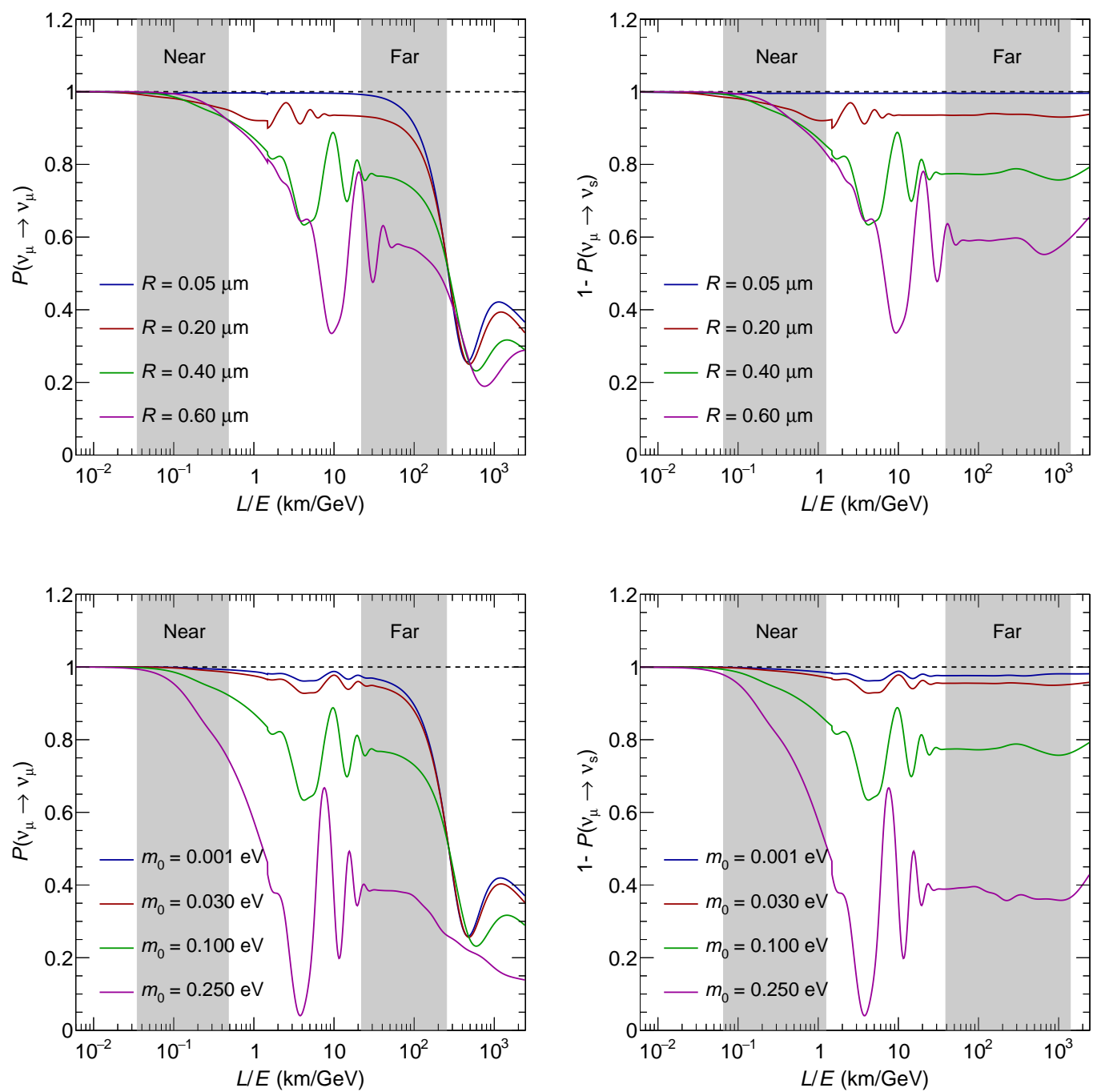

Figure 2.9: Effect of $R$ (top) and $m_{0}$ (bottom) on the muon disappearance probability (left) and the sterile appearance probability (right) in the MINOS detectors, taking energy resolution effects into account. For the top plots, $m_{0}$ is fixed to $0.100 \mathrm{eV}$, while for the bottom plots $R$ is fixed to $0.40 \mu \mathrm{m}$. The other oscillation parameters are fixed for all cases: $\Delta m_{32}^{2}=2.37 \times 10^{-3} \mathrm{eV}^{2}$, $\theta_{23}=0.695, \theta_{13}=0.149, \delta_{\mathrm{CP}}=0, \Delta m_{21}^{2}=7.54 \times 10^{-5} \mathrm{eV}^{2}$, and $\theta_{12}=0.588$. The $L / E$ coverage of the ND and FD are represented by the grey bands, which contain $90 \%$ of the MINOS and MINOS+ events between 0 and $40 \mathrm{GeV}$. 

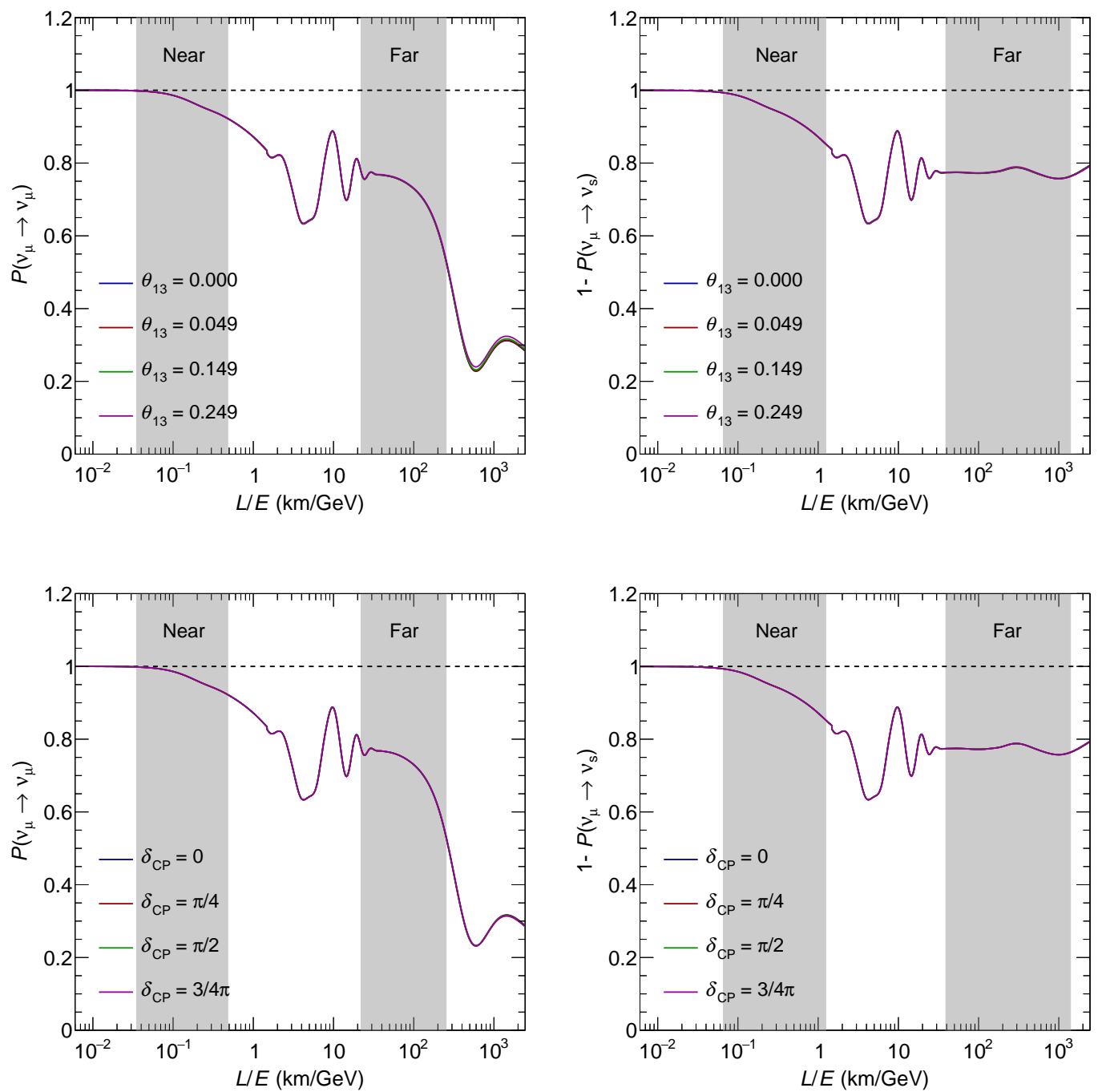

Figure 2.10: Effect of $\theta_{13}$ (top) and $\delta_{\mathrm{CP}}$ (bottom) on the muon disappearance probability (left) and the sterile appearance probability (right) in the MINOS detectors, taking energy resolution effects into account. For the top plots, $\delta_{\mathrm{CP}}$ is fixed to 0 , while for the bottom plots $\theta_{13}$ is fixed to 0.149 . The other oscillation parameters are fixed for all cases: $R=0.40 \mu \mathrm{m}, m_{0}=0.100 \mathrm{eV}$, $\Delta m_{32}^{2}=2.37 \times 10^{-3} \mathrm{eV}^{2}, \theta_{23}=0.695, \Delta m_{21}^{2}=7.54 \times 10^{-5} \mathrm{eV}^{2}$, and $\theta_{12}=0.588$. The $L / E$ coverage of the ND and FD are represented by the grey bands, which contain $90 \%$ of the MINOS and MINOS+ events between 0 and $40 \mathrm{GeV}$. 

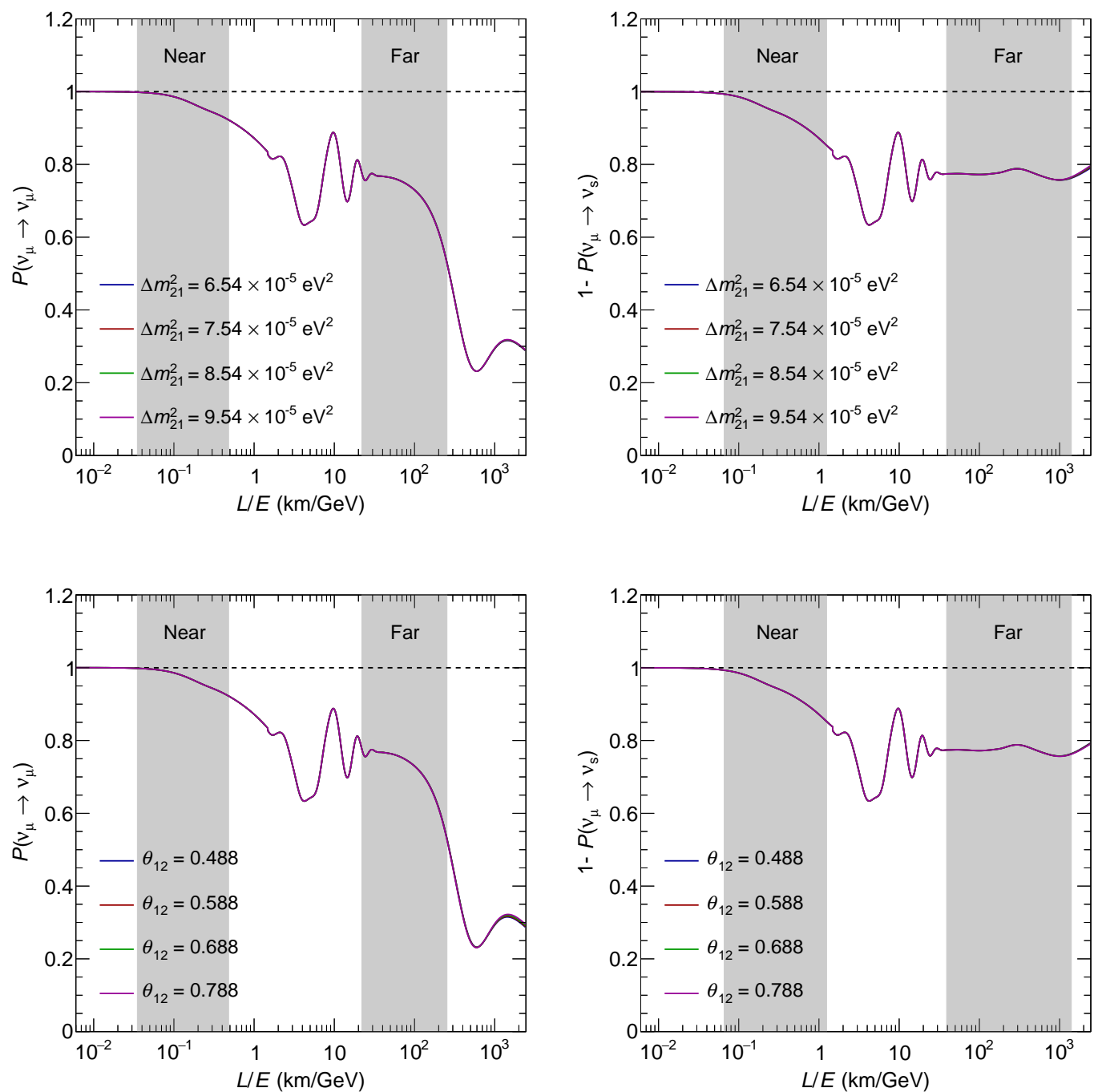

Figure 2.11: Effect of $\Delta m_{21}^{2}$ (top) and $\theta_{12}$ (bottom) on the muon disappearance probability (left) and the sterile appearance probability (right) in the MINOS detectors, taking energy resolution effects into account. For the top plots, $\theta_{12}$ is fixed to 0.588 , while for the bottom plots $\Delta m_{21}^{2}$ is fixed to $7.54 \times 10^{-5} \mathrm{eV}^{2}$. The other oscillation parameters are fixed for all cases: $R=0.40 \mu \mathrm{m}, m_{0}=0.100 \mathrm{eV}, \Delta m_{32}^{2}=2.37 \times 10^{-3} \mathrm{eV}^{2}, \theta_{23}=0.695$, $\theta_{13}=0.149$, and $\delta_{\mathrm{CP}}=0$. The $L / E$ coverage of the ND and FD are represented by the grey bands, which contain $90 \%$ of the MINOS and MINOS+ events between 0 and $40 \mathrm{GeV}$. 


\subsection{Interpreting the Large Extra Dimensions Model}

The fact that only two extra parameters, $R$ and $m_{0}$, are required in addition to the three-flavor mixing angles and mass squared splittings is in sharp contrast with the apparent mathematical complexity of the LED model.

Ignoring matter effects, the KK mixing matrix elements appearing in Formulas (2.4) and (2.5) can be written as [63]

$$
\begin{aligned}
\left(W_{i}^{(0 n)}\right)^{2} & =\frac{2}{1+\pi^{2} \xi_{i}^{2} / 2+2\left(\lambda_{i}^{(n)}\right)^{2} / \xi_{i}^{2}} \\
W_{i}^{(k n)} & =\frac{k \xi_{i}}{\left(\lambda_{i}^{(\mathrm{n})}\right)^{2}-k^{2}} W_{i}^{(0 n)}
\end{aligned}
$$

for $n=0, \ldots,+\infty$ and $n=1, \ldots,+\infty$ and where $\xi_{i}=\sqrt{2} m_{i} R(i=1,2,3)$. The eigenvalues $\lambda_{i}^{(n)}$ of the Hamiltonian are solutions of the equation

$$
\lambda_{i}^{(n)}-\frac{\pi}{2} \xi_{i}^{2} \cot \left(\pi \lambda_{i}^{(n)}\right)=0
$$

Treating the effects of mixing of the sterile modes with the zero modes in the KK towers as small perturbations on top of the three-flavor oscillations between the zero modes of the KK towers, the expressions for $\lambda_{i}^{(n)}$ and $W_{i}^{(0 n)}$ simplify to

$$
\begin{aligned}
\lambda_{i}^{(0)} & =\frac{\xi_{i}}{\sqrt{2}}\left(1-\frac{\pi^{2}}{12} \xi_{i}^{2}+\mathrm{O}\left(\xi_{i}^{4}\right)\right), \\
\lambda_{i}^{(k)} & =k+\frac{1}{2 k} \xi_{i}^{2}+\mathrm{O}\left(\xi_{i}^{4}\right), \\
W_{i}^{(00)} & =1-\frac{\pi^{2}}{12} \xi_{i}^{2}+\mathrm{O}\left(\xi_{i}^{4}\right),
\end{aligned}
$$




$$
\begin{aligned}
& W_{i}^{(0 k)}=\frac{\xi_{i}}{k}+\mathrm{O}\left(\xi_{i}^{3}\right), \\
& W_{i}^{(k 0)}=-\frac{\xi_{i}}{k}+\mathrm{O}\left(\xi_{i}^{3}\right), \\
& W_{i}^{(k k)}=1-\frac{\xi_{i}}{2 k^{2}}+\mathrm{O}\left(\xi_{i}^{4}\right), \\
& W_{i}^{(j k)}=\mathrm{O}\left(\xi_{i}^{2}\right) \text { for } j \neq k=1, \ldots,+\infty .
\end{aligned}
$$

These approximations are valid in the limit of small $\xi_{i}$ or in other words for $R^{-1} \gg m_{0}$ (unit conversion: $1 \mu \mathrm{m}=5 \mathrm{eV}^{-1}$ ). Figure 2.12 shows the values of $\xi_{1}, \xi_{2}$, and $\xi_{3}$ in the $\left(R, m_{0}\right)$ plane assuming normal ordering. In the following discussions about model interpretations it is assumed that $\xi_{i}<0.3$ such that Formulas (2.10)-(2.16) are not used in the region of the $\left(R, m_{0}\right)$ plane where $\xi_{3}>0.3$.

It should be noted that these approximations are not used in the LED analyses presented in Chapter 5 and Chapter 6. These approximations are only employed to increase the understanding of the LED model. 

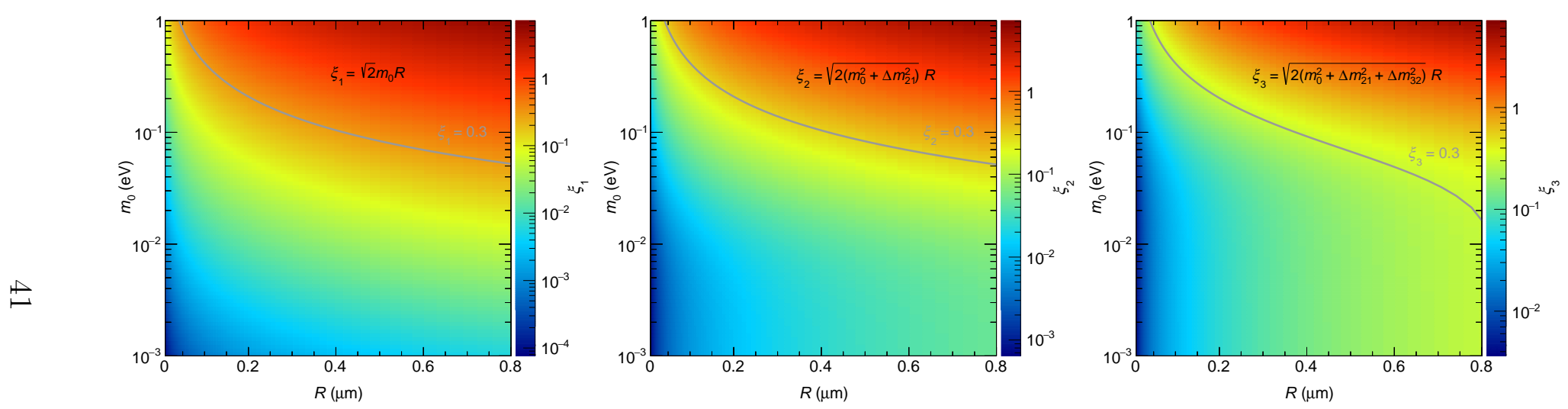

3

Figure 2.12: The values of $\xi_{1}=\sqrt{2} m_{1} R$ (left), $\xi_{2}=\sqrt{2} m_{2} R$ (middle), and $\xi_{3}=\sqrt{2} m_{3} R$ (right) in the $\left(R, m_{0}\right)$ plane assuming normal mass ordering. 


\subsubsection{A Back-of-the-Envelope Calculation of the Sensitivity to $R$}

In the small $\xi_{i}$ approximation, the amplitude corresponding to the muon disappearance probability in Formula (2.4) to leading order in $\xi_{i}$ is

$$
\begin{aligned}
\mathcal{A}\left(v_{\alpha} \rightarrow v_{\alpha}\right) & \approx \sum_{j=1}^{3}\left|U_{\alpha j}\right|^{2} \exp \left(i m_{j}^{2} \frac{L}{2 E}\right)+\sum_{j=1}^{3} \sum_{k=1}^{+\infty}\left|U_{\alpha j}\right|^{2}\left(\frac{\xi_{j}}{k}\right)^{2} \exp \left[i\left(\frac{k}{R}\right)^{2} \frac{L}{2 E}\right] \\
& \approx \mathcal{A}_{\mathrm{SM}}\left(v_{\alpha} \rightarrow v_{\alpha}\right)+\mathcal{A}_{\mathrm{LED}}\left(v_{\alpha} \rightarrow v_{\alpha}\right),
\end{aligned}
$$

where the first term can be identified as the SM contribution and the second term quantifies the effect of the sterile neutrino towers in the extra dimensions. From the second term in Formula (2.17) it follows that a $10 \%$ effect due to LED requires at least one $\xi_{i}^{2}$ to be of the order of 0.1. From $\Delta m_{32}^{2}=2.37 \times 10^{-3} \mathrm{eV}^{2}$ it follows that at least one neutrino has a mass of about $0.05 \mathrm{eV}\left(m_{3}=\sqrt{\Delta m_{32}^{2}} \approx 0.05 \mathrm{eV}\right.$ for $\left.m_{0}=0 \mathrm{eV}\right)$, which for $\xi_{i}^{2} \approx 0.1$ corresponds to $R \approx 1 \mu \mathrm{m}$ :

$$
R=\sqrt{\frac{\xi_{i}^{2}}{2 m_{i}^{2}}} \approx \sqrt{\frac{0.1}{2(0.05 \mathrm{eV})^{2}}} \approx 4.5 \mathrm{eV}^{-1} \approx 1 \mu \mathrm{m} .
$$

\subsubsection{Mass Ordering for Disappearance Channels}

It was already demonstrated in Fig. 2.7 that the mass ordering has no significant effect on the muon neutrino disappearance and sterile appearance probabilities in the MINOS detectors. In the small $\xi_{i}$ limit this can also be understood qualitatively, as shown below for the muon disappearance channel.

From Formula (2.17) it follows that

$$
\mathcal{A}_{\mathrm{LED}}\left(v_{\mu} \rightarrow v_{\mu}\right) \propto \xi_{1}^{2}\left|U_{\mu 1}\right|^{2}+\xi_{2}^{2}\left|U_{\mu 2}\right|^{2}+\xi_{3}^{2}\left|U_{\mu 3}\right|^{2} .
$$


The absolute masses of the active neutrinos are

$$
\begin{array}{rlrl} 
& \text { Normal Ordering (NO) } & & \text { Inverted Ordering (IO) } \\
m_{1}=m_{0} & m_{3}=m_{0} \\
m_{2}=\sqrt{m_{0}^{2}+\Delta m_{21}^{2}} & m_{1}=\sqrt{m_{0}^{2}+\left|\Delta m_{32}^{2}\right|-\Delta m_{21}^{2}} \\
m_{3}=\sqrt{m_{0}^{2}+\Delta m_{21}^{2}+\left|\Delta m_{32}^{2}\right|} & m_{2}=\sqrt{m_{0}^{2}+\left|\Delta m_{32}^{2}\right|}
\end{array}
$$

For small values of $m_{0}, m_{3} \gg m_{2} \approx m_{1}$ for normal ordering and $m_{2} \approx m_{1} \gg$ $m_{3}$ for inverted ordering. Furthermore, $m_{3}^{(\mathrm{NO})} \approx m_{2}^{(\mathrm{IO})}$. Note that for large values of $m_{0}$ the masses become degenerate in both ordering schemes, removing the difference between normal and inverted ordering.

If, to simplify matters, tribimaximal mixing [80] is assumed such that

$$
\left(\begin{array}{ccc}
\left|U_{\mathrm{e} 1}\right|^{2} & \left|U_{\mathrm{e} 2}\right|^{2} & \left|U_{\mathrm{e} 3}\right|^{2} \\
\left|U_{\mu 1}\right|^{2} & \left|U_{\mu 2}\right|^{2} & \left|U_{\mu 3}\right|^{2} \\
\left|U_{\tau 1}\right|^{2} & \left|U_{\tau 2}\right|^{2} & \left|U_{\tau 3}\right|^{2}
\end{array}\right)=\left(\begin{array}{ccc}
\frac{2}{3} & \frac{1}{3} & 0 \\
\frac{1}{6} & \frac{1}{3} & \frac{1}{2} \\
\frac{1}{6} & \frac{1}{3} & \frac{1}{2}
\end{array}\right),
$$

then

$$
\begin{aligned}
\mathcal{A}_{\mathrm{LED}}^{(\mathrm{NO})}\left(v_{\mu} \rightarrow v_{\mu}\right) & \propto\left(\xi_{3}^{(\mathrm{NO})}\right)^{2}\left|U_{\mu 3}\right|^{2} \approx \frac{1}{2}\left(\xi_{3}^{(\mathrm{NO})}\right)^{2} \\
\mathcal{A}_{\mathrm{LED}}^{(\mathrm{IO})}\left(v_{\mu} \rightarrow v_{\mu}\right) & \propto\left(\xi_{1}^{(\mathrm{IO})}\right)^{2}\left|U_{\mu 1}\right|^{2}+\left(\xi_{2}^{(\mathrm{IO})}\right)^{2}\left|U_{\mu 2}\right|^{2} \\
& \approx \frac{1}{6}\left(\xi_{1}^{(\mathrm{IO})}\right)^{2}+\frac{1}{3}\left(\xi_{2}^{(\mathrm{IO})}\right)^{2} \\
& \approx\left(\frac{1}{6}+\frac{1}{3}\right)\left(\xi_{2}^{(\mathrm{IO})}\right)^{2} \\
& \approx \frac{1}{2}\left(\xi_{3}^{(\mathrm{NO})}\right)^{2} .
\end{aligned}
$$

Note that for electron neutrino disappearance, the effect of LED is significantly larger for inverted ordering because of the suppression of $\xi_{3}$ due to the small value of $\left|U_{\mathrm{e} 3}\right|^{2}=\sin ^{2} \theta_{13}[64]$. 


\subsubsection{A Closer Look at the Kaluza-Klein Towers}

In the small $\xi_{i}$ approximation, the masses of the sterile neutrinos in the KK towers are given by

$$
\begin{aligned}
& m_{\mathrm{KK}, 1}^{(k)}=\frac{\lambda_{1}^{(k)}}{R}=\frac{k}{R}+m_{0}^{2} \frac{R}{k}+\mathrm{O}\left(\frac{\xi_{1}^{4}}{R}\right) \\
& m_{\mathrm{KK}, 2}^{(k)}=\frac{\lambda_{2}^{(k)}}{R}=m_{\mathrm{KK}, 1}^{(k)}+\Delta m_{21}^{2} \frac{R}{k}+\mathrm{O}\left(\frac{\xi_{2}^{4}}{R}\right) \\
& m_{\mathrm{KK}, 3}^{(k)}=\frac{\lambda_{3}^{(k)}}{R}=m_{\mathrm{KK}, 1}^{(k)}+\left(\Delta m_{21}^{2}+\Delta m_{32}^{2}\right) \frac{R}{k}+\mathrm{O}\left(\frac{\xi_{3}^{4}}{R}\right) .
\end{aligned}
$$

Figure 2.13 shows the difference between the first KK mass and the active neutrino mass in each tower, $m_{\mathrm{KK}, i}^{(1)}-m_{i}$, as a function of $R$ and $m_{0}$, as well as the mass squared splittings between the first KK mode and the active modes, $\Delta m_{\mathrm{KK}, i}^{2}=\left(m_{\mathrm{KK}, i}^{(1)}\right)^{2}-m_{i}^{2}$. The difference between the three towers is small, which is even more apparent from Fig. 2.14 and 2.15 where for selected points in the $\left(R, m_{0}\right)$ plane the active mode and first six KK modes, calculated using Formulas (2.26)-(2.28), are shown for each tower. The KK modes in the different towers are aligned and their absolute mass is mostly determined by the inverse of $R$ (see Fig. 2.14) and has very little dependency on $m_{0}$ (see Fig. 2.15). The absolute mass of the active modes depends only on $m_{0}$ as is apparent from Formula (2.19) and as can be seen in Fig. 2.14 and 2.15. Figures 2.14 and 2.15 also show the corresponding muon disappearance probabilities at a baseline of $735 \mathrm{~km}$, with and without the MINOS energy resolution taken into account. For $R=0.01 \mu \mathrm{m}$, the $\mathrm{KK}$ modes have masses of $20 \mathrm{eV}$ and higher which is too far away from the active modes to cause any 
significant effects on top of the three-flavor disappearance. With increasing $R$, the distance between the KK modes and active modes decreases and oscillations between them occur, causing a shift of the oscillation minimum to lower energies, an overall decrease in the survival probability, and the appearance of modulations on the survival probability because of fast oscillations to the KK states. Away from the three-flavor minimum, the amplitude of the oscillations increases with increasing values of $R$ and $m_{0}$, while the frequency of the modulations decreases with increasing values of $R$ and has no dependency on $m_{0}$ for small $\xi_{i}$. 

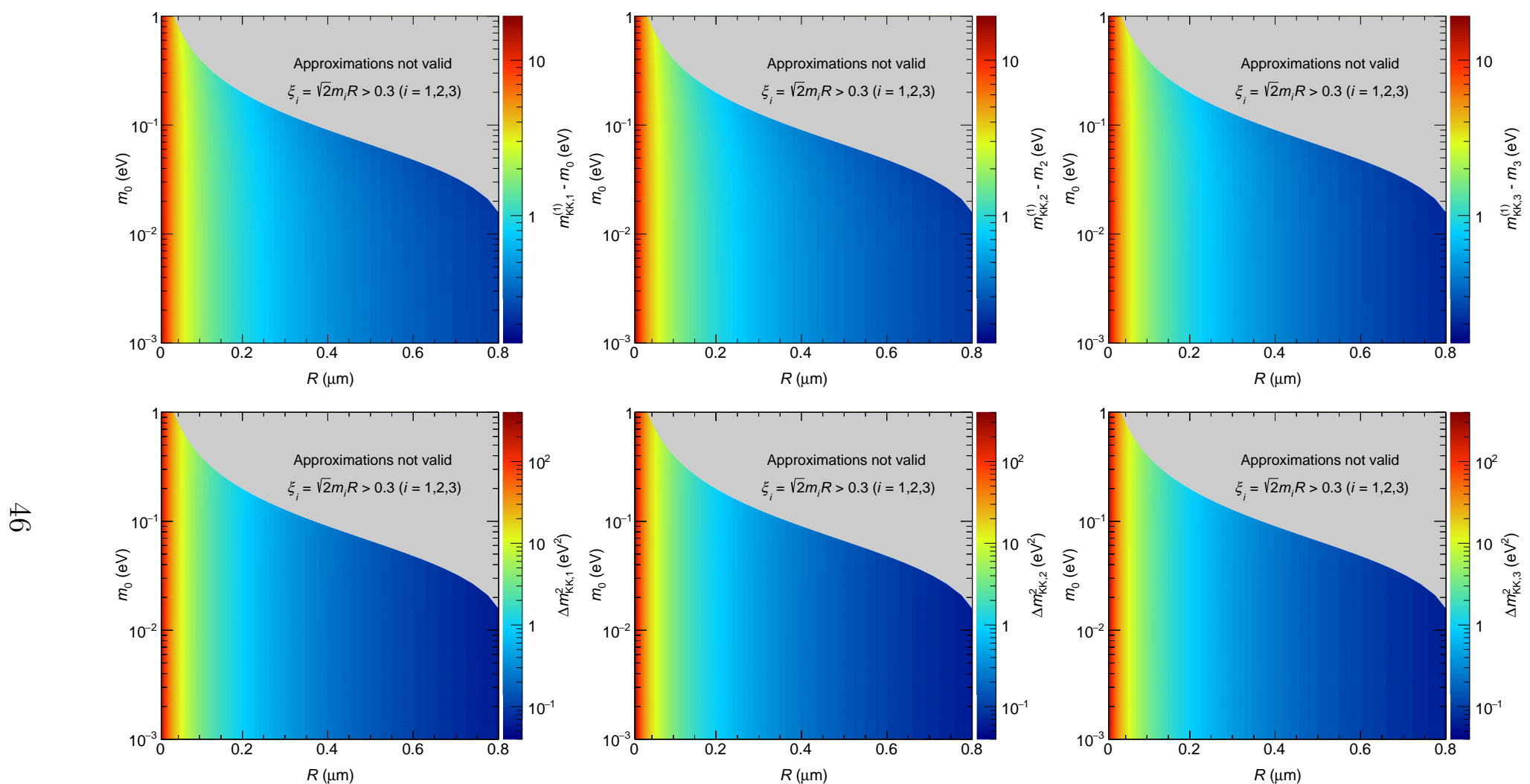

Figure 2.13: The difference between the first KK mass and the active neutrino mass (top) and the difference between the square of the first KK mass and the square of the active neutrino mass (bottom) in each tower in the $\left(R, m_{0}\right)$ plane for the small $\xi_{i}$ approximation and assuming normal mass ordering. 

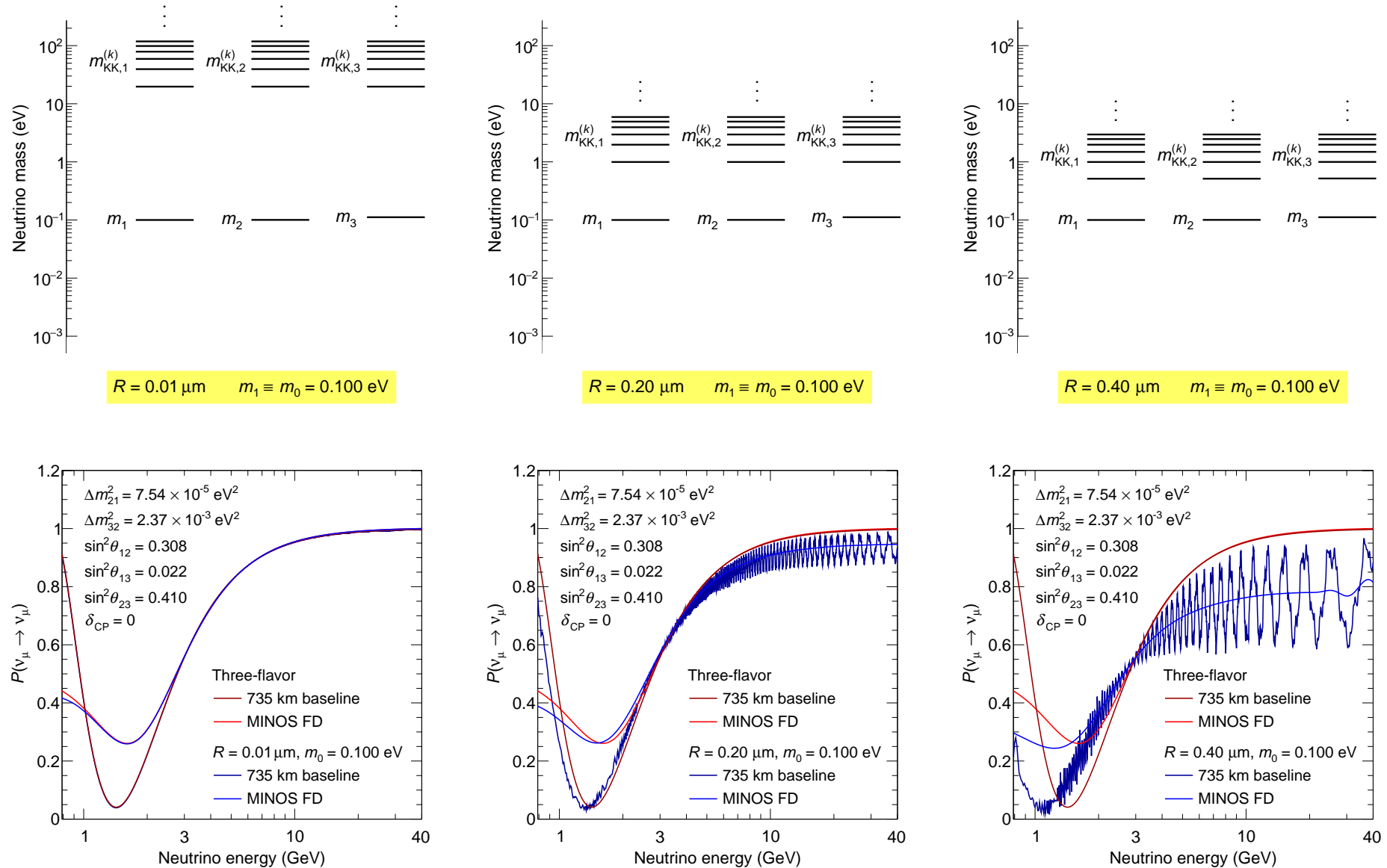

Figure 2.14: Effect of the LED parameter $R$ on the neutrino masses and oscillation probabilities. (Top) For fixed $m_{0} \equiv m_{1}=0.100 \mathrm{eV}$ (normal mass ordering), the effect of increasing $R$ on the active neutrino masses $m_{1}, m_{2}$, and $m_{3}$ and on the sterile neutrino masses in the KK towers $m_{\mathrm{KK}, i}^{(k)}$ (with $i=1,2,3$ and $k=1, \ldots,+\infty)$ is demonstrated. The absolute masses of the active neutrinos are determined by $m_{0}$ and the known mass squared splittings $\Delta m_{32}^{2}$ and $\Delta m_{21}^{2}$, while the absolute masses of the sterile neutrinos are approximately proportional to the inverse of $R$. (Bottom) Comparison of the three-flavor (red) $v_{\mu}$ disappearance probability with the three LED (blue) cases from (top). With respect to the three-flavor case, the LED probabilities show a displacement of the oscillation minimum, a reduction of the integrated survival probability, and modulations due to fast oscillations with the sterile neutrinos in the KK towers. The effect of the CC energy resolution on the oscillation probability in the MINOS FD is shown as well. 

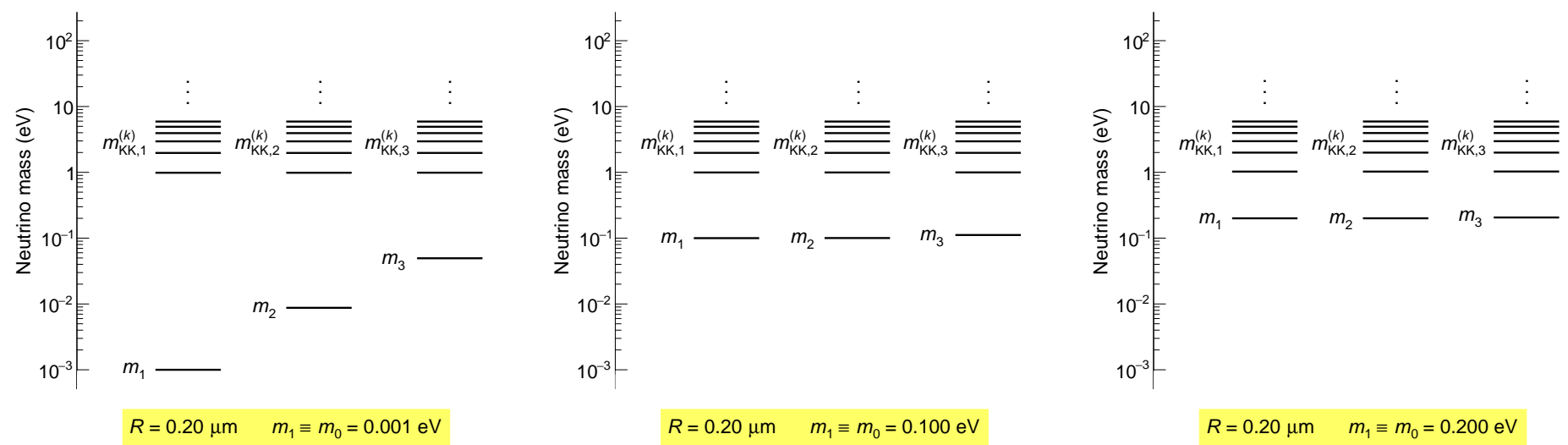

$R=0.20 \mu \mathrm{m} \quad m_{1} \equiv m_{0}=0.001 \mathrm{eV}$
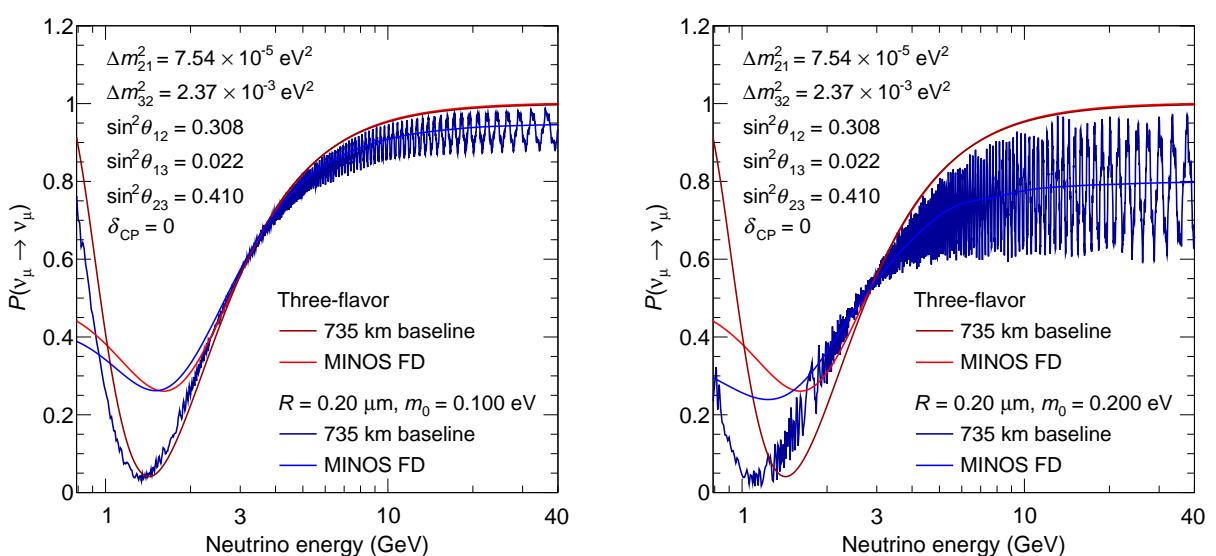

Figure 2.15: Effect of the LED parameter $m_{0}$ on the neutrino masses and oscillation probabilities. (Top) For fixed $R=0.2 \mu \mathrm{m}$ (normal mass ordering), the effect of increasing $m_{0}$ on the active neutrino masses $m_{1}$, $m_{2}$, and $m_{3}$ and on the sterile neutrino masses in the KK towers $m_{\mathrm{KK}, i}^{(k)}$ (with $i=1,2,3$ and $\left.k=1, \ldots,+\infty\right)$ is demonstrated. The absolute masses of the active neutrinos are determined by $m_{0}$ and the known mass squared splittings $\Delta m_{32}^{2}$ and $\Delta m_{21}^{2}$, while the absolute masses of the sterile neutrinos are approximately proportional to the inverse of $R$. (Bottom) Comparison of the three-flavor (red) $v_{\mu}$ disappearance probability with the three LED (blue) cases from (top). With respect to the three-flavor case, the LED probabilities show a displacement of the oscillation minimum, a reduction of the integrated survival probability, and modulations due to fast oscillations with the sterile neutrinos in the KK towers. The effect of the CC energy resolution on the oscillation probability in the MINOS FD is shown as well. 


\subsubsection{Defining an Effective Mixing Angle}

To quantify the effect on the muon disappearance probability of mixing of the sterile modes with the active modes in all three towers, an effective mixing angle can be defined (P. Machado, personal communication, May 2017) from the unitarity relation in the LED model

$$
\begin{aligned}
\mathrm{SM}: & \delta_{\alpha \beta}=\sum_{i=1}^{3} U_{\alpha i} U_{\beta i}^{*}, \\
\text { LED: } & \delta_{\alpha \beta}=\sum_{i=1}^{3} \sum_{n=0}^{+\infty} U_{\alpha i} W_{i}^{(0 n)} U_{\beta i}^{*} W_{i}^{(0 n)} .
\end{aligned}
$$

Note that for $R \rightarrow 0, W_{i}^{(0 n)} \rightarrow 1$ for $n=0$ and $W_{i}^{(0 n)} \rightarrow 0$ for $n>0$ such that the unitarity relation (2.29) of the SM is retrieved. Using Formula (2.30) for the muon neutrino disappearance channel

$$
\begin{aligned}
& 1=\sum_{i=1}^{3} \sum_{n=0}^{+\infty}\left|U_{\mu i}\right|^{2}\left(W_{i}^{(0 n)}\right)^{2} \\
& =\underbrace{\sum_{i=1}^{3}\left|U_{\mu i}\right|^{2}\left(W_{i}^{(00)}\right)^{2}}_{\text {SM-like }}+\underbrace{\sum_{i=1}^{3} \sum_{n=1}^{+\infty}\left|U_{\mu i}\right|^{2}\left(W_{i}^{(0 n)}\right)^{2}}_{\text {LED-like }} \\
& =\cos ^{2} \theta_{\text {eff }}^{\mu \mu}+\sin ^{2} \theta_{\text {eff }}^{\mu \mu},
\end{aligned}
$$

where in the second step the unitarity relation is broken down in a sum of an SM-like term and an LED-like term, which leads to the definition of the effective LED mixing angle for the muon disappearance channel, $\theta_{\mathrm{eff}}^{\mu \mu}$, for which

$$
\sin ^{2} \theta_{\mathrm{eff}}^{\mu \mu}=1-\cos ^{2} \theta_{\mathrm{eff}}^{\mu \mu} \equiv 1-\sum_{i=1}^{3}\left|U_{\mu i}\right|^{2}\left(W_{i}^{(00)}\right)^{2} .
$$




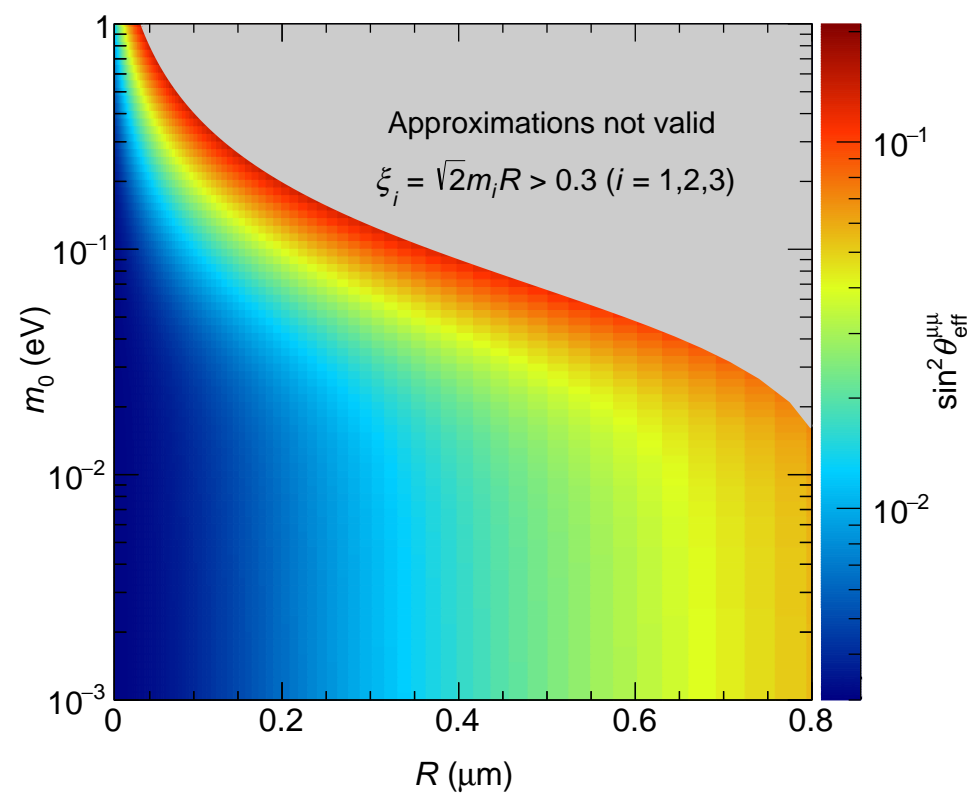

Figure 2.16: The effective mixing angle as $\sin ^{2} \theta_{\text {eff }}^{\mu \mu}$ in the $\left(R, m_{0}\right)$ plane assuming normal mass ordering.

Note that the definition of $\theta_{\text {eff }}^{\mu \mu}$ in Formula (2.34) does not involve any approximations and quantifies mixing between active modes and all the KK modes in the muon disappearance channel such that

$$
P_{\mathrm{LED}}\left(v_{\mu} \rightarrow v_{\mu}\right) \approx P_{\mathrm{SM}}\left(v_{\mu} \rightarrow v_{\mu}\right)\left(1-\frac{1}{2} \sin ^{2} 2 \theta_{\mathrm{eff}}^{\mu \mu}\right)
$$

For $R \rightarrow 0$, the effective mixing angle goes to zero such that the SM probability is retrieved. In the small $\xi_{i}$ approximation, the mixing angle can be calculated as a function of $R$ and $m_{0}$ as shown in Fig. 2.16. Because $\frac{1}{2} \sin ^{2} 2 \theta_{\text {eff }}^{\mu \mu} \approx 2 \sin ^{2} \theta_{\text {eff }}^{\mu \mu}$, the values of $\sin ^{2} \theta_{\text {eff }}^{\mu \mu}$ should be multiplied by 2 to get an estimate of the mixing due to LED at a fixed point in the $\left(R, m_{0}\right)$ plane. 


\section{Chapter 3}

\section{The MINOS and MINOS+ Experiments}

The MINOS (Main Injector Neutrino Oscillation Search) [81] experiment was a long-baseline accelerator beam neutrino oscillation experiment designed to perform precision measurements of muon neutrino disappearance. Two functionally identical magnetized steel-scintillator sampling calorimeters were placed on the axis of the Neutrinos at the Main Injector (NuMI) neutrino beam [82]. The $0.98 \mathrm{kT}$ Near Detector (ND) is located $1.04 \mathrm{~km}$ from the NuMI production target at Fermilab, Illinois, and was used to monitor the composition of the NuMI beam. The $5.4 \mathrm{kT}$ Far Detector (FD) was located $735 \mathrm{~km}$ from the production target in the Soudan Underground Mine, Minnesota. MINOS collected accelerator beam neutrino and atmospheric neutrino data from 2003 until 2012 and was succeeded by the MINOS+ [83] experiment in 2013, which collected data until 2016 using a different NuMI neutrino beam configuration. Between observing the first atmospheric neutrino event in the FD in July 2003 and collecting the last NuMI beam neutrino event in June 2016, MINOS and MINOS+ collected $2.38 \times 10^{21}$ protons on target (POT) of accelerator beam neutrino data and 60.9 kt-yrs of atmospheric neutrino data. The measured neutrino energy spectra in both detectors are used to determine neutrino oscillation parameters within and beyond the standard three-flavor 
neutrino oscillation paradigm $[30,47,48,84-88]$ and to study neutrino interactions $[89,90]$.

This chapter gives a brief review of the NuMI neutrino beam, followed by a discussion of the MINOS detectors and the type of neutrino interactions observed by MINOS and MINOS+. The data collected by MINOS and MINOS+ and employed in this dissertation to study the LED model are discussed in Section 3.4. Section 3.5 summarizes the calibration and longterm performance of the detectors, while the final two sections of this chapter discuss simulation and event reconstruction, respectively.

\subsection{The NuMI Neutrino Beam}

The NuMI neutrino beam [82] at Fermilab was built to provide a neutrino beam for the MINOS experiment. Figure 3.1 shows a schematic of the NuMI beamline. During MINOS and MINOS+ operation, $120 \mathrm{GeV}$ protons from the Main Injector at Fermilab were directed towards a graphite target. The hadrons produced in the proton-target interactions were focused by two magnetic horns and decayed into muon neutrinos in a $675 \mathrm{~m}$ long decay volume

$$
\begin{aligned}
& \pi^{ \pm} \rightarrow \mu^{ \pm}+v_{\mu} / \bar{v}_{\mu}, \\
& \mathrm{K}^{ \pm} \rightarrow \mu^{ \pm}+v_{\mu} / \bar{v}_{\mu}, \\
& \mu^{ \pm} \rightarrow \mathrm{e}^{ \pm}+v_{\mathrm{e}} / \bar{v}_{\mathrm{e}}+v_{\mu} / \bar{v}_{\mu},
\end{aligned}
$$

where Formula (3.3) represents the decay of the tertiary muons from Formulas (3.1) and (3.2). 


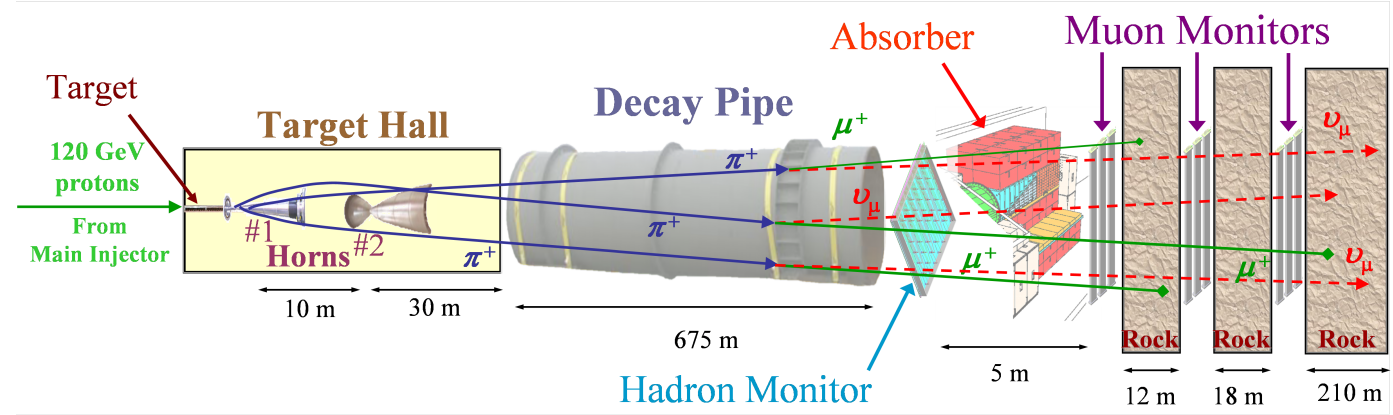

Figure 3.1: A schematic of the NuMI neutrino beam, showing the graphite target, the magnetic horns, the decay pipe, the hadron monitor, and the muon shielding. This figure is taken from Ref. [82].

Changing the configuration of the magnetic horns changes the energy spectrum of the neutrino beam. The target was moved closer to the first horn and the second horn was moved further away from the first horn at the start of the MINOS+ era, resulting in a higher neutrino energy beam. Changing the current direction in the magnetic horns changes the charge sign of the focused hadrons and leads to either a $v_{\mu}$ or $\bar{v}_{\mu}$ beam. This is illustrated in Fig. 3.2. The beam composition at the ND when operating in $v_{\mu}$ mode consisted of $91.7 \%(97.5 \%) v_{\mu}, 7 \%(1.8 \%) \bar{v}_{\mu}$, and $1.3 \%(0.7 \%) v_{\mathrm{e}}$ and $\bar{v}_{\mathrm{e}}$ during the MINOS (MINOS+) era. When operating in $\bar{v}_{\mu}$ mode during the MINOS era, the beam composition was $58.1 \% v_{\mu}, 39.9 \% \bar{v}_{\mu}$, and $2.0 \% v_{\mathrm{e}}$ and $\bar{v}_{\mathrm{e}}$. The presence of $\bar{v}_{\mu}$ in the $v_{\mu}$ beam and $v_{\mu}$ in the $\bar{v}_{\mu}$ beam is due to high energy hadrons that are not defocused by the magnetic horns. The $\bar{v}_{\mu}$ beam as measured in the ND is less pure than the $\boldsymbol{v}_{\mu}$ beam mainly because of smaller antineutrino interaction cross sections. 

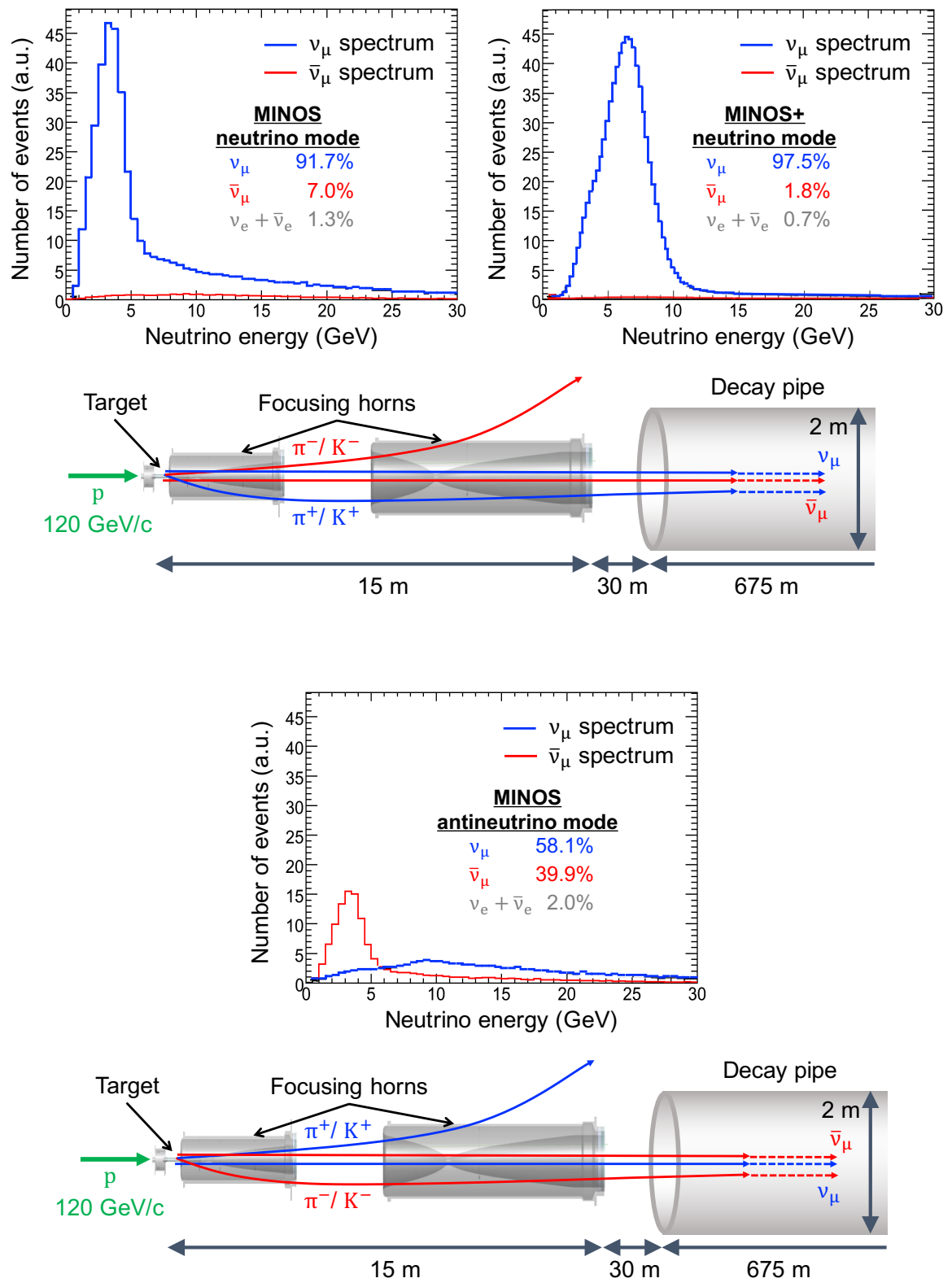

Figure 3.2: Creating a muon neutrino or antineutrino beam with NuMI. (Top) When positive pions and kaons are focused into the decay pipe by the magnetic horns, their decays lead to a neutrino beam dominated by muon neutrinos. For MINOS, the target was inserted $50.4 \mathrm{~cm}$ into the first horn to maximize the neutrino flux in the $1-3 \mathrm{GeV}$ range. For MINOS+, the target was moved outside the first horn and the second horn was moved further away from the first horn, $19.2 \mathrm{~m}$ from the upstream end of the first horn, leading to a neutrino flux peaking at 6-7 GeV. (Bottom) Focusing negative pions and kaons by reversing the horn current leads to a neutrino beam dominated by muon antineutrinos. This configuration was only adopted in the MINOS era. 


\section{$3.2 \quad$ The MINOS Detectors}

Figure 3.3 shows a schematic representation of the MINOS detectors along the NuMI beamline. The MINOS detectors had similar designs to minimize systematic uncertainties arising from the NuMI beam flux [91] and neutrino interaction cross sections. Each detector was comprised of toroidally magnetized $2.54 \mathrm{~cm}$ thick steel planes interleaved with polystyrene-based scintillator [92] planes. The magnetic fields, averaging to $1.28 \mathrm{~T}$ and $1.42 \mathrm{~T}$ in the fiducial volume of the ND and FD [81], respectively, allowed to distinguish neutrino and antineutrino interactions on an event-by-event basis. The FD was made up of two modules, axially separated by a $1.15 \mathrm{~m}$ gap.

Each scintillating plane consisted of $4.1 \mathrm{~cm}$ wide, $1.0 \mathrm{~cm}$ thick strips placed side-by-side with lengths up to $3.75 \mathrm{~m}$ in the ND and $8 \mathrm{~m}$ in the FD. A total of 11,616 strips divided over 153 planes were used in the ND, and 93,120 strips made up 485 planes in the FD. The strips in each alternate scintillator plane were rotated by $90^{\circ}$ to allow for three-dimensional track reconstruction. The two plane rotations are named $\mathrm{U}$ and $\mathrm{V}$ planes. For each strip, a coextruded $\mathrm{TiO}_{2}$ cladding maximized internal reflection and light collection was optimized using a wavelength shifting (WLS) fiber running down the center of its length. Clear acrylic fibers on both sides of the FD strips and on one side of the ND strips transmitted the light to Hamamatsu multianode photomultiplier tubes (PMTs). Figure 3.4 shows the structure of one scintillator strip and its readout system. The ND employed 192 M64 PMTs [93], and 1452 M16 PMTs 
were used in the FD [94]. A cosmic ray veto shield covered the top and sides of the FD to reduce the background in atmospheric neutrino measurements.

Different PMT readout electronics were employed for both detectors because of the very different rates of cosmic muon and neutrino interactions in the ND and FD. The ND was required to read out multiple events per beam spill and therefore designed to allow continuous readout. The ND readout [95] integrated charge for each PMT pixel in intervals of $19 \mathrm{~ns}$, corresponding to the NuMI beam RF structure of $53 \mathrm{MHz}$, while outside of beam spills, for cosmic ray or light injection events, a set of eight samples from each PMT pixel were read out when triggered by the dynode tap.

In contrast, the low interaction rates in the FD, with a few beam events per day and one cosmic muon event every two seconds, allowed an 8-fold multiplexing of non-neighboring scintillating strips on each of the PMT pixels [96] and each PMT was read out only when triggered by the PMT dynode tap. The readout systems were regularly recalibrated and therefore much more linear and stable than the PMTs.

\subsection{Neutrino Interactions in the MINOS Detectors}

As mentioned in Section 2.3, the two relevant oscillation channels in this dissertation are the muon disappearance channel and the sterile neutrino appearance channel, probed by $\mathrm{CC}$ and $\mathrm{NC}$ events, respectively, observed in the MINOS ND and FD. 

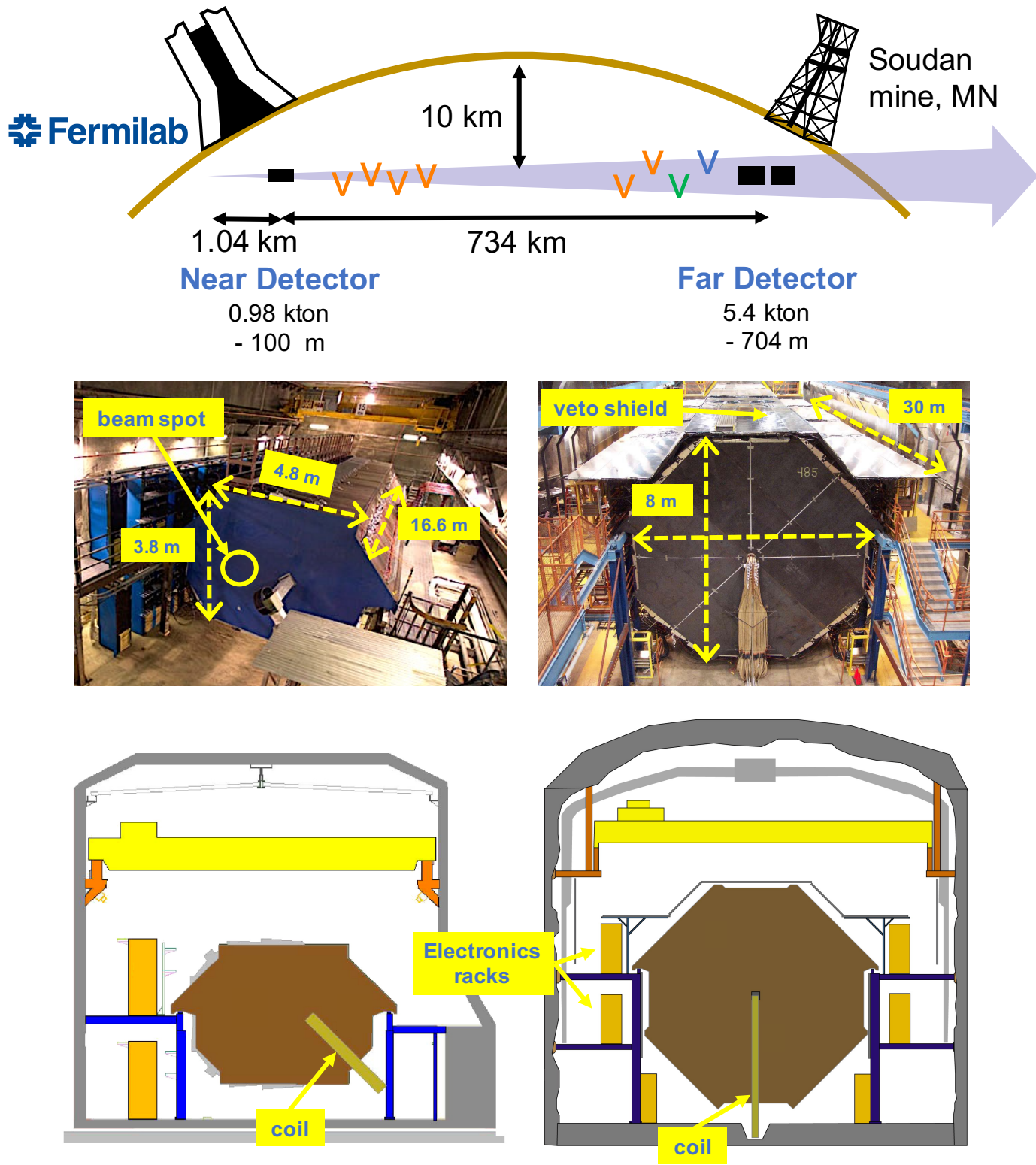

Figure 3.3: The MINOS and MINOS+ long-baseline neutrino oscillation experiments. The experiments consisted of a Near Detector located $1.04 \mathrm{~km}$ from the NuMI beam target at Fermilab, Illinois, and a Far Detector located $734 \mathrm{~km}$ further in the Soudan Underground Mine, Minnesota. Both detectors were magnetized steel-scintillator sampling-tracking calorimeters positioned on the NuMI beamline axis. The detector pictures and bottom drawings are taken from Ref. [81]. 

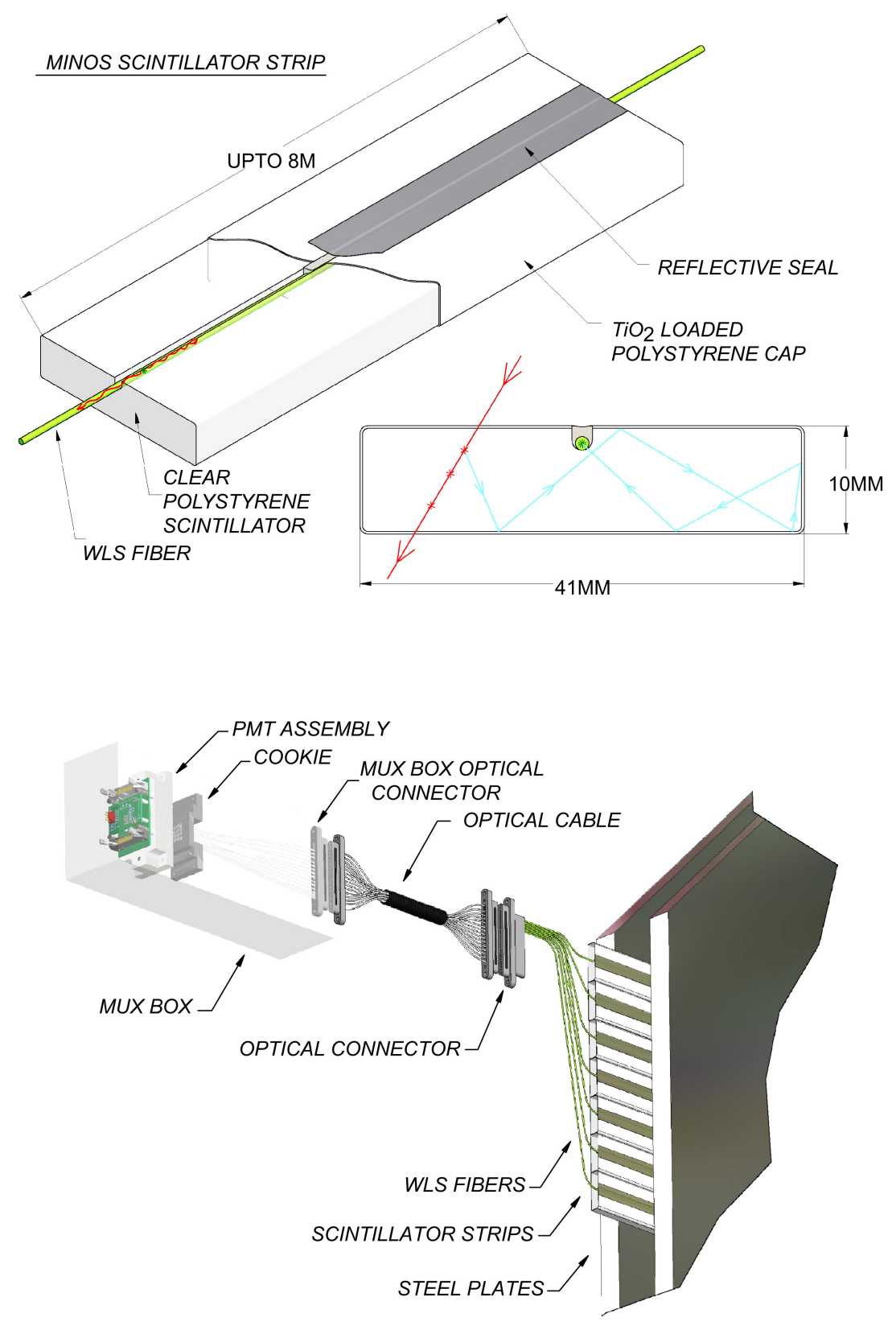

Figure 3.4: The structure of a MINOS scintillator strip (top) and an illustration of the readout of a scintillator plane (bottom). Scintillation light produced by an ionizing particle is reflected inside the strip by the $0.25 \mathrm{~mm}$-thick outer reflective coating. When the light is absorbed, the WLS fiber reemits it isotropically. The wavelength-shifted photons with a direction within the total internal reflection cones are transported along the fiber toward the edges of the scintillator strip. The light is then carried by a clear optical fiber to a pixel of the PMT. These figures are taken from Ref. [81] and were made by M. Proga. 
Figure 3.5 shows an example of a simulated $\mathrm{CC}$ event in the MINOS detectors and its corresponding Feynman diagram. A $v_{\mu} \mathrm{CC}$ event in the MINOS detectors is characterized by a single outgoing muon track accompanied by possible hadronic showers near the interaction vertex. Event reconstruction in the MINOS detectors is discussed in Section 3.7. There are three dominant types of $\mathrm{CC}$ interactions in the $0-40 \mathrm{GeV}$ neutrino energy range considered in this dissertation:

- quasi-elastic scattering dominates below $1 \mathrm{GeV}$, where typically a small or no hadronic shower component is observed in the MINOS detectors

$$
\begin{aligned}
& v_{\mu} \mathrm{n} \rightarrow \mu^{-} \mathrm{p}, \\
& \bar{v}_{\mu} \mathrm{p} \rightarrow \mu^{+} \mathrm{n} ;
\end{aligned}
$$

- resonance production gains importance at a few $\mathrm{GeV}$, where the struck nucleon $(\mathrm{N})$ is excited to a baryon resonance $\left(\mathrm{N}^{*}\right)$ which most likely decays to a nucleon-pion final state

$$
\begin{gathered}
v_{\mu} \mathrm{N} \rightarrow \mu^{-} \mathrm{N}^{*} \\
\mathrm{~N}^{*} \rightarrow \pi \mathrm{N}^{\prime}
\end{gathered}
$$

and for which the pion can give a shower-like topology if it undergoes hadronic interactions or a track-like signature (not for $\pi^{0}$ ); and

- deep inelastic scattering (DIS) dominates above a few $\mathrm{GeV}$, where the neutrino scatters off the quarks inside the nucleons leading to a large hadronic shower. 
Figure 3.6 shows an example of a simulated NC event in the MINOS detectors and its corresponding Feynman diagram. A $v_{\alpha}(\alpha=\mathrm{e}, \mu, \tau) \mathrm{NC}$ event in the MINOS detectors has a short diffuse hadronic shower and possibly short hadron tracks. The scattered incoming neutrino is not observed.

\subsection{MINOS and MINOS+ Data}

The LED analyses presented in this dissertation employ MINOS and MINOS+ accelerator beam data corresponding to exposures of $10.56 \times 10^{20}$ POT and $5.80 \times 10^{20}$ POT, respectively, and collected with the NuMI beam operating in $v_{\mu}$ mode. During the MINOS era, an additional exposure of $3.36 \times 10^{20}$ POT was collected for the NuMI beam operating in $\bar{v}_{\mu}$ mode. The final year of MINOS+ data corresponds to $3.89 \times 10^{20}$ POT collected in $v_{\mu}$ mode and is not used in this dissertation. The exposure is shown as a function of time in the left panel of Fig. 3.7. The beam data collected by MINOS corresponds to a neutrino energy spectrum peaking at $3 \mathrm{GeV}$, as shown in the right panel of Fig. 3.7 , as it was designed to perform precision measurements of the atmospheric neutrino oscillation parameters $\Delta m_{32}^{2}$ and $\theta_{23}$ within the three-flavor paradigm. This peak energy shifted to $7 \mathrm{GeV}$ during the MINOS+ era to increase the number of neutrino events at higher energies where beyond the three-flavor paradigm models typically predict larger effects. The main result of the Far-over-Near LED analysis presented in Chapter 5 employs the MINOS beam data only, while the first two years of MINOS+ beam data are included in the two-detector LED analysis presented in Chapter 6. 

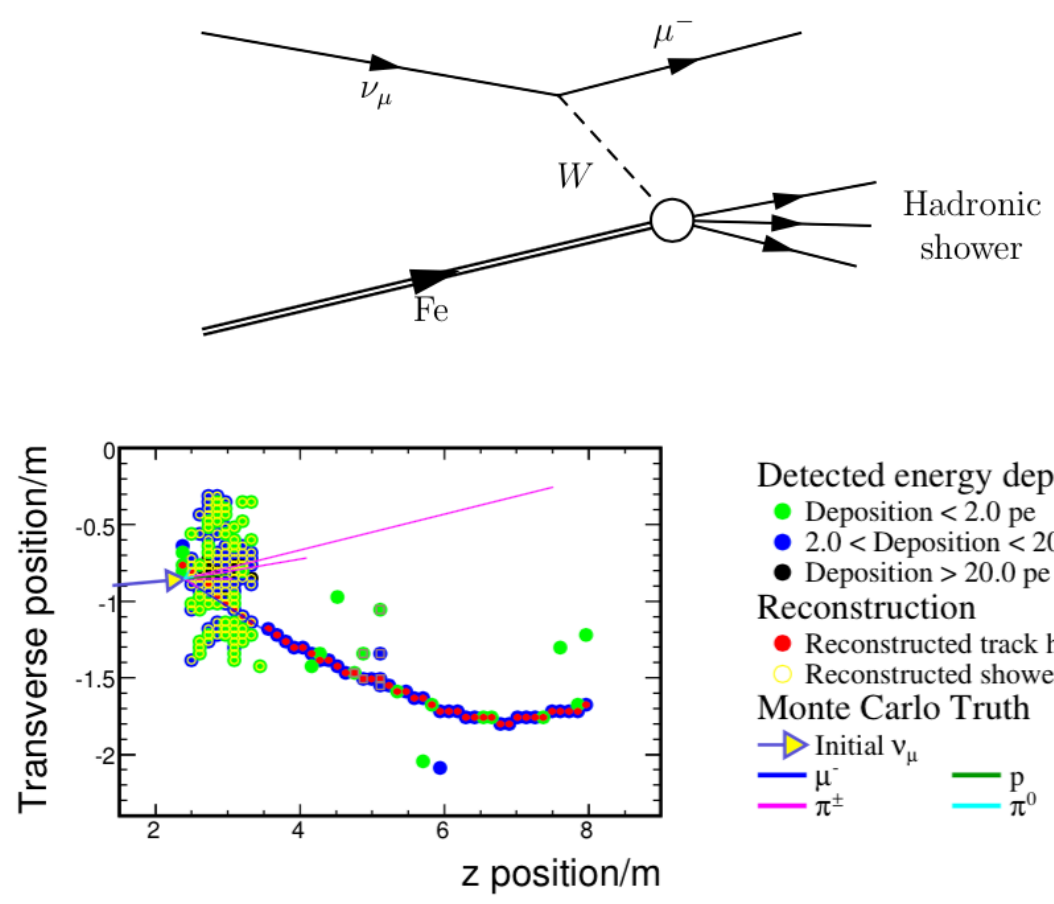

Detected energy depositions

- Deposition $<2.0$ pe

- $2.0<$ Deposition $<20.0$ pe

- Deposition $>20.0$ pe

Reconstruction

- Reconstructed track hit

Reconstructed shower hit

Monte Carlo Truth

$\rightarrow$ Initial $v_{\mu}$

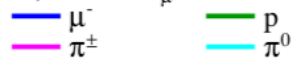

Figure 3.5: Feynman diagram of a $\mathrm{CC}$ interaction between a $v_{\mu}$ and iron nucleus (top) and an example of a simulated event display in the MINOS detectors (bottom). The colored dots represents the magnitude of the signals in the scintillator strips and whether they have been reconstructed as part of a muon track or a shower. The outgoing muon forms a long track used to identify the $\mathrm{CC}$ interaction. The curvature of the track is caused by the magnetic field in the detector and is used to determine the electric charge sign of the muon. These figures are taken from Ref. [97]. 

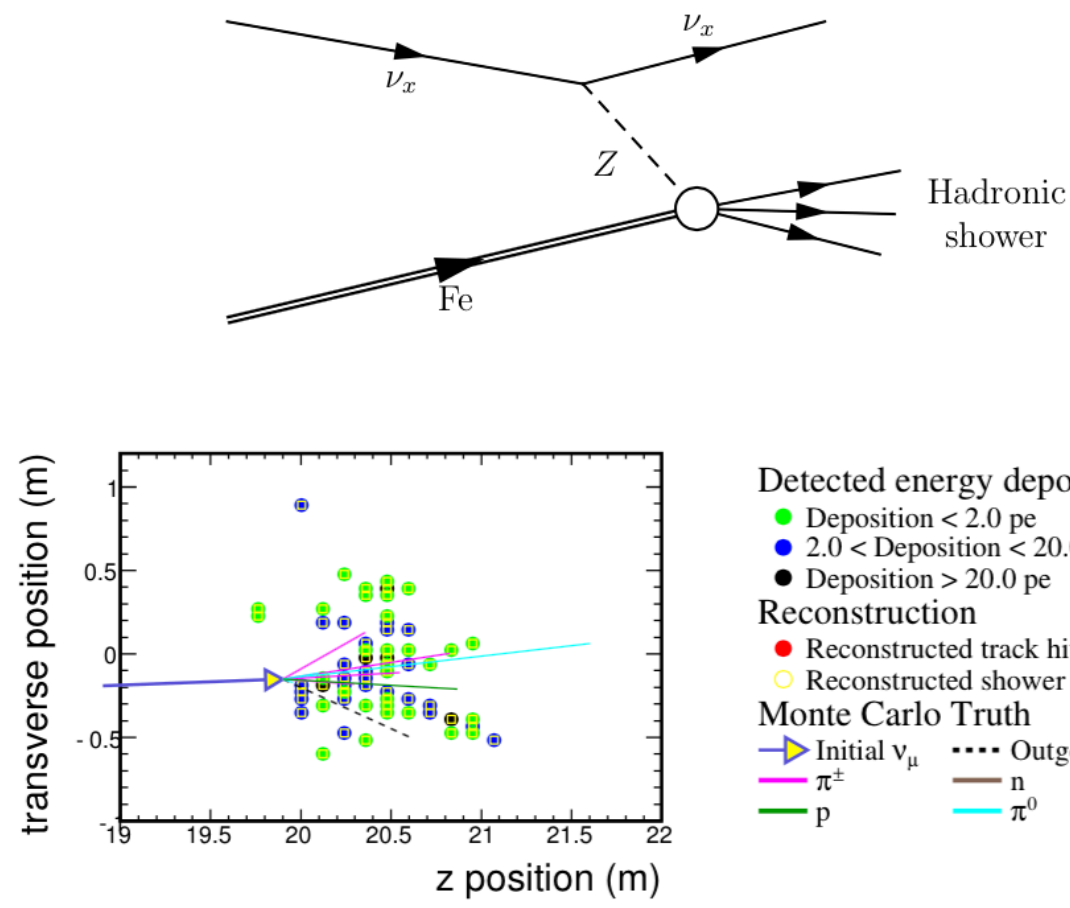

Detected energy depositions

- Deposition $<2.0$ pe

- $2.0<$ Deposition $<20.0$ pe

- Deposition $>20.0$ pe

Reconstruction

- Reconstructed track hit Reconstructed shower hit Monte Carlo Truth

$\rightarrow$ Initial $v_{\mu} \quad \cdots$ Outgoing $v$

$\begin{array}{ll}\pi^{ \pm} & \mathrm{n} \\ \mathrm{p} & -\pi^{0}\end{array}$

Figure 3.6: Feynman diagram of an $\mathrm{NC}$ interaction between a $v_{\alpha}$ and iron nucleus (top) and an example of a simulated event display in the MINOS detectors (bottom). The colored dots represents the magnitude of the signals in the scintillator strips and whether they have been reconstructed as part of a muon track or a shower. There are typically no long tracks in an NC event. These figures are taken from Ref. [97]. 

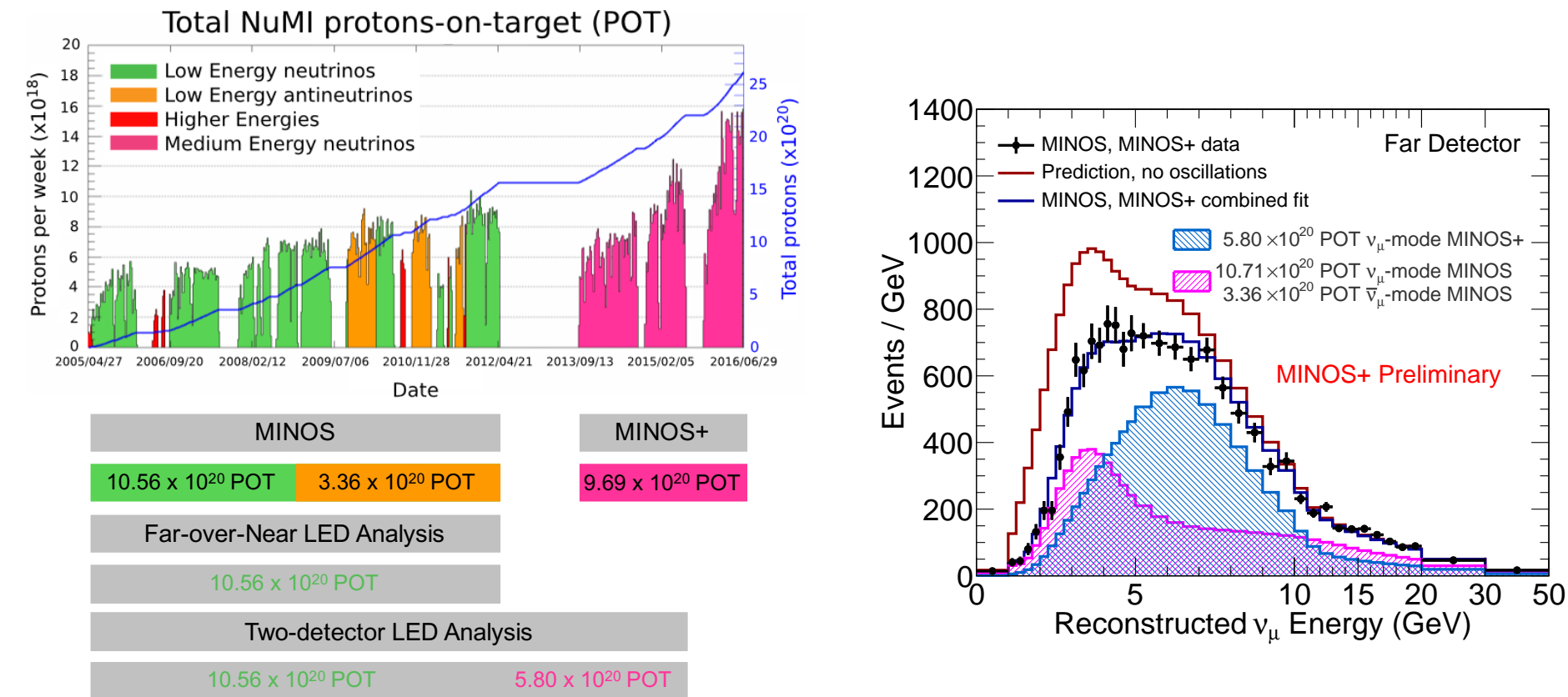

Reconstructed $v_{\mu}$ Energy $(\mathrm{GeV})$

Figure 3.7: The MINOS and MINOS+ accelerator beam neutrino data. (Left) The data in terms of protons on target collected by MINOS and MINOS+ from March 2005 until July 2016. The data exposures used for the Far-over-Near and two-detector LED analyses presented in this dissertation are indicated at the bottom. (Right) The full CC MINOS dataset and the first two years of MINOS+ data (black points) as a function of reconstructed neutrino energy at the FD. The data is compared to the three-flavor paradigm prediction (blue line) and the no oscillations prediction (red line). The simulated contributions of the MINOS and MINOS+ CC samples are shown by the magenta and cyan hatched histograms, respectively. The right figure is taken from Ref. [98]. 


\subsection{Detector Calibration and Performance}

A calibration technique was developed $[81,99]$ and employed to remove the spatial and temporal variations in the detector response which would otherwise degrade the calorimetric energy resolution and therefore limit the ability of MINOS and MINOS+ to perform precision measurements.

\subsubsection{Calibration Procedure}

The aim of the calibration procedure is to remove temporal and spatial variations of detector response within each detector, relate particle energy deposits between the different detectors, and translate the detector response to energy units of GeV. To this end, an in situ optical Light Injection (LI) system, cosmic ray muon events, and a calibration detector were used.

A multistage procedure converts a raw pulse height $Q_{\text {raw }}(s, x, t, d)$ as a function of strip number $s$, position $x$, time $t$, and detector $d$ into a fully corrected signal $Q_{\text {corr }}$

$$
\begin{aligned}
Q_{\text {corr }}= & Q_{\text {raw }}(s, x, t, d) \times D(d, t) \times L\left(d, s, Q_{\text {raw }}\right) \\
& \times S(d, s, t) \times A(d, s, x, t) \times M(d),
\end{aligned}
$$

where $L\left(d, s, Q_{\text {raw }}\right)$ is the linearity correction for the electronics response as a function of pulse height, $D(d, t)$ is the drift correction which accounts for the variation in detector response with time, $S(d, s, t)$ is the strip-to-strip correction which removes differences in response between the strip centers, and $A(d, s, x, t)$ is the attenuation correction which corrects for the attenuation of 
light in the WLS fiber along the length of the strip. An overall scale factor, $M(d)$, converts the corrected pulse height into the same energy unit for all detectors, the Muon Energy Unit (MEU).

In addition to the above calibration chain, a FD strip-by-strip timing calibration improves reconstruction of cosmic ray and atmospheric neutrino events and a PMT single photoelectron (PE) calibration aided in adjusting PMT thresholds and eliminating crosstalk.

The LI system measured the readout behavior of both detectors and was used for linearity corrections, single PE gain calibration, and monitoring the stability of PMTs and electronics over time. The LI system injected light from pulsed ultraviolet light-emitting diodes [100] into the end of the scintillator modules just prior to the interface between the WLS and clear fiber to mimic the signals that come from particle energy deposits in the scintillator. The intensity of the injected light was monitored by PIN photodiodes.

Cosmic ray muon tracks were used to measure the response of the scintillator as a function of position and time in each detector and as such were employed for the FD timing calibration, the drift correction, the stripto-strip correction, the attenuation correction, and to determine the relative energy calibration constants $M(d)$.

The MINOS Calibration Detector (CalDet) [99], a functionally identical scaled down version of the ND and FD, was exposed to various test beams at CERN between 2001 and 2003 to measure the detector response to protons, 
pions, electrons, and muons with energies between 0.2 and $10 \mathrm{GeV}$, allowing the determination of the absolute energy scale for the MINOS detectors. The relative energy calibration is performed by normalizing the measured response of the ND and FD to that of CalDet.

\subsubsection{Long Term Performance of the Detectors}

During MINOS and MINOS+ operation, the detector and PMT response in the ND and FD were frequently monitored and a uniform calibration scheme was applied. These data were used to measure the stability of the response of the MINOS detectors over time. The effects of hall conditions such as temperature fluctuations were also quantified. In what follows, detector response refers to the combined response of the scintillator, WLS fibers, PMTs, and electronics; PMT response refers to the combined response of the PMTs and electronics; and PMT gain refers to the combined response of the PMTs and electronics to one PE.

\subsubsection{Detector Drift}

The drift of the detector response was monitored daily using throughgoing cosmic ray muons and was found to decrease gradually with time in both detectors, as shown in the top panel of Fig. 3.8. Over the whole course of MINOS and MINOS+ operation, the raw response of the detector decreased by roughly $11 \%$ in the ND and $18 \%$ in the FD. The daily drift correction $D(d, t)$ was determined by comparing the median of the total pulse height per 
plane to the median response determined on Dec. $1^{\text {st }}, 2005$

$$
D(d, t)=\frac{\text { Median } \operatorname{response}\left(d, t_{0}\right)}{\text { Median } \operatorname{response}(d, t)}
$$

\subsubsection{PMT Behavior}

To determine the PMT gains, each ND (FD) strip end was pulsed 1000 (300) times per hour [81] by the LI system at approximately 50 PEs per pulse. To average out daily fluctuations, the data were summed over a three day interval and the gains were evaluated using photon statistics [99]. The middle panel of Fig. 3.8 shows the ND and FD gains as a function of time. Since November 2004, the gains increased by $28 \%$ and $20 \%$ in the ND and FD, respectively.

The short term fluctuations in gains were well correlated with temperature and more pronounced in the ND data because of larger temperature variations. The ND PMT temperatures were taken from the temperature sensors nearest to them in the data acquisition racks. The temperature of the FD PMTs closely followed the FD cavern temperature.

To quantify the linearity of the PMT response, each strip end was pulsed 1000 times every 28 days by the LI system at 40 intervals between about 10 and 200 PE. At light levels beyond 100 PE the PMTs were markedly nonlinear. The M64 PMTs in the ND had a response that is roughly quadratic with charge, reaching a nonlinearity of about 5\% at 100 PE. For the M16 PMTs

in the FD, the nonlinearity of the readout electronics dominated that of the 
PMTs due to their larger pixel size. The combined readout and PMT system was fit to a pole-and-kicker function that is very linear until about $100 \mathrm{PE}$ and then rapidly turns over to become about $20 \%$ nonlinear at about $200 \mathrm{PE}$. These numbers represent crude averages and in practice every channel was individually linearized each month.

\subsubsection{Detector Light Yield}

Since the detector response was a product of the PMT response and the amount of light incident on the PMT photocathodes, the changes in light level as a function of time were calculated as the ratio of the detector response to the PMT gains. This measurement was affected by the scintillator light yield, the attenuation in the WLS and clear fibers, and the optical transparency of the readout chain. The bottom panel of Fig. 3.8 shows the light level for both detectors as a function of time. The overall light level in both detectors dropped approximately $30 \%$ in 11.5 years. The light level curve is smoother than the drift curve because it removes the large variations due to PMT gain.

As with the gain measurement, the rate at which the light level dropped decreased with time. Short term variations in the light level were due to a variety of sources, including detector and hardware instabilities and temperature

variations. For both detectors, the scintillator temperature followed that of the detector hall. 

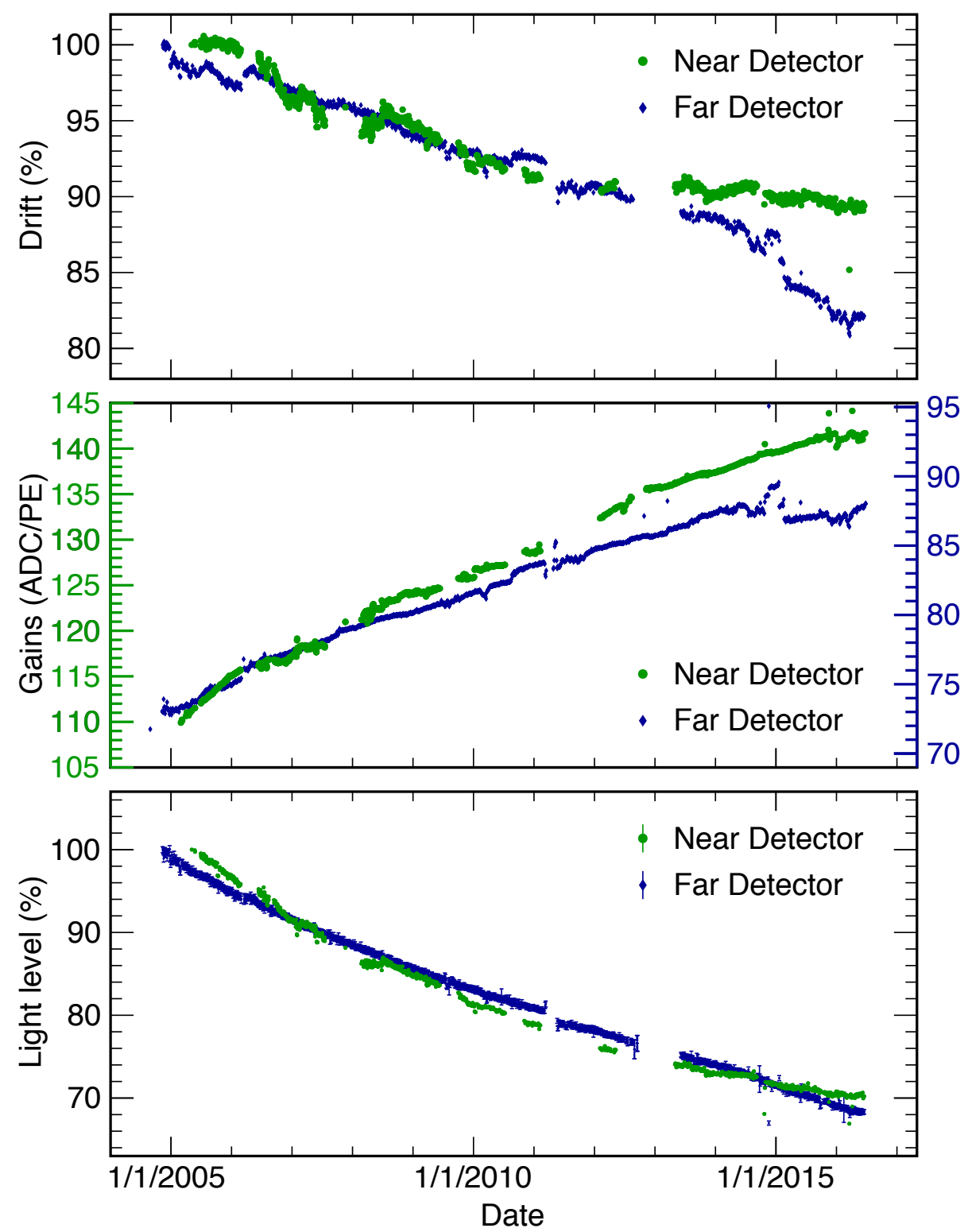

Figure 3.8: Long term behavior of the MINOS Near (green points) and Far (blue points) Detectors. (Top) The daily change in detector response normalized to the first data for each detector. (Middle) The measured PMT gains, averaged over three day intervals. (Bottom) The relative light level, defined as the ratio of the detector response to the PMT gains, normalized to the first data for each detector. 


\subsubsection{Far Detector Timing Calibration}

The FD readout system was synchronized only to within $30 \mathrm{~ns}$ due to different readout cable lengths and channel-to-channel variations of the readout electronics. A well-calibrated FD timing system allowed to distinguish upward-going muons from downward-going muons resulting in atmospheric neutrino events, as well as for vetoing cosmic muon events.

The timing calibration employed an iterative process to correct for three effects: the increased delay in the arrival of the first photon for smaller signals, time offset differences on a channel-by-channel basis, and jumps in these time offsets caused by occasional hardware swaps.

High energy through-going cosmic muon events, with tracks crossing at least 20 scintillator planes and for which the RMS deviation from a straight line is smaller than $1 \mathrm{~cm}$ such that the cosmic muons can be assumed to travel at the speed of light, were used to calculate the characteristic time offset for the East and West ends of each FD scintillator strip. The time differences between East and West ends were used to validate the calibration and to estimate the timing resolution at a single strip end to be about $0.3 \mathrm{~ns}$ [101], as illustrated in Fig. 3.9. Figure 3.10 shows the distribution of timing calibration constants for all FD strip ends from the start of atmospheric neutrino measurements in 2003 until the last beam neutrino event in 2016, divided into one year periods. The timing calibration constants were reasonably stable over time. 

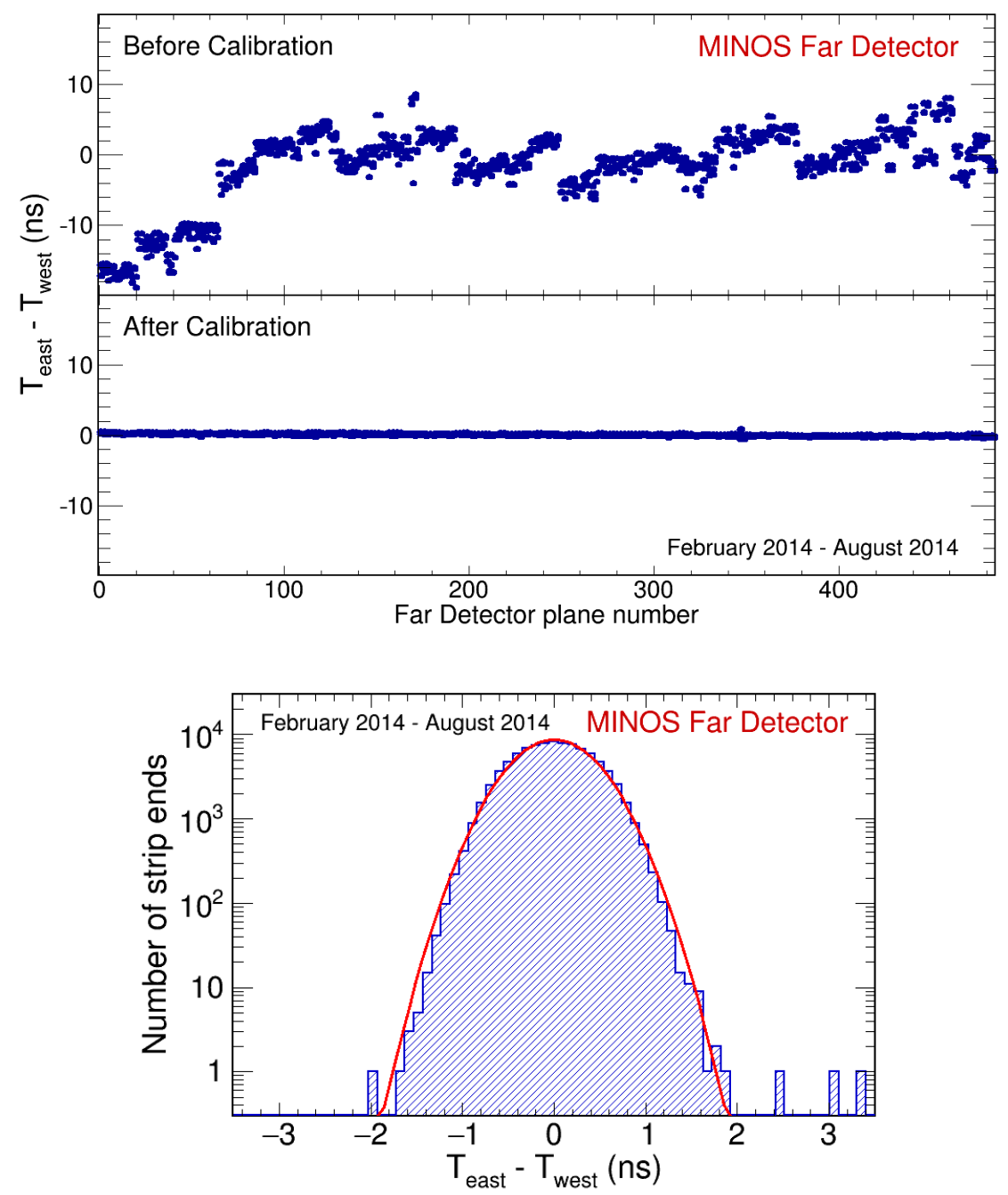

Figure 3.9: (Top) The average time difference between East and West strip end recordings of through-going cosmic muon hits as a function of the plane number in the Far Detector, before and after timing calibration is applied, for a six month period in 2014. For each plane the time differences are averaged across all strips within that plane. The piecewise pattern observed before calibration is applied is due to the readout system setup. (Bottom) The distribution of calibrated East-West differences for all strips, which follows a Gaussian distribution with standard deviation of $0.40 \mathrm{~ns}$. These figures are taken from Ref. [102]. 


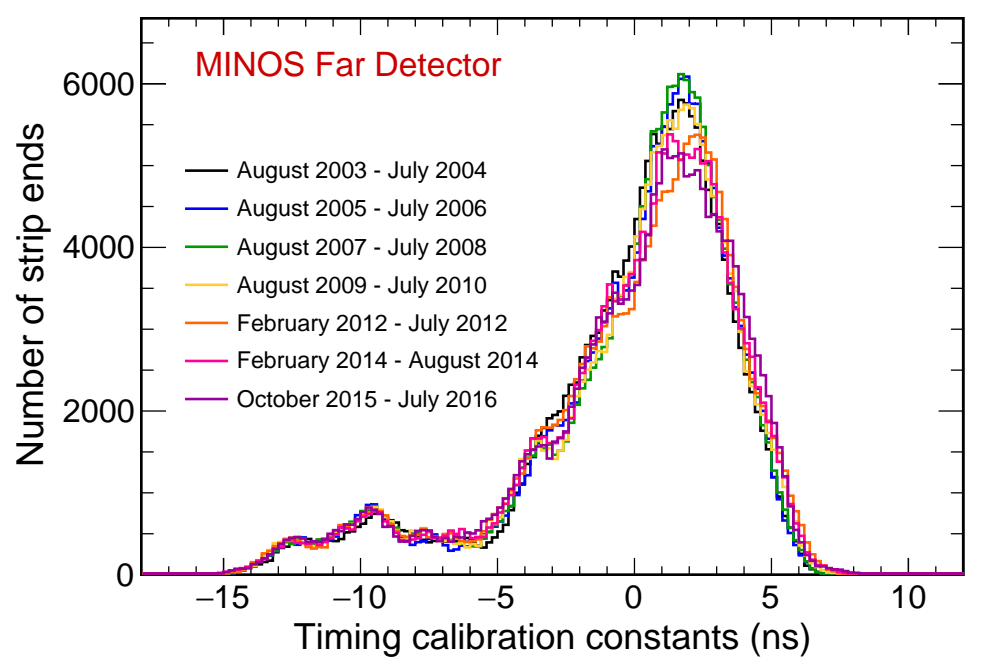

Figure 3.10: The distribution of timing calibration constants for all Far Detector strip ends for different one year intervals. This figure is taken from Ref. [102].

\subsubsection{Normalizing the Scintillator Response}

The strip-to-strip calibration normalizes the response to through-going cosmic ray muons at the center of a single strip to that of the detector average. The calibration constant $S_{i}$ of strip $i$ is defined as:

$$
S_{i}=\frac{\langle R\rangle}{R_{i}} \frac{S_{0}}{\operatorname{median}(\langle S\rangle)}
$$

where $R_{i}$ and $\langle R\rangle$ are the strip and average detector response, respectively, after drift and linearity corrections are applied. In both detectors the strip response was approximately Gaussian with an RMS of about 30\%. The spread in response was due to the light yield of the scintillator, the length of the scintillating strips and WLS fibers, and the gain of the PMTs. The width of the 
response increased at a rate of approximately $1 \%$ per year, dominated by the aging of the PMTs, and increased the median value of the calibration constant. This is accounted for in the strip-to-strip calibration through the ratio of the desired normalization value $S_{0}$ and the median calibration constant.

Through-going cosmic ray muon events were also used to normalize the response within a strip to the response at its center. A sum of two exponentials is used to model the attenuation $A(d, s, x, t)$ behavior within each strip [81]

$$
A(x)=A_{1} \exp \left(-\frac{x}{L_{1}}\right)+A_{2} \exp \left(-\frac{x}{L_{2}}\right),
$$

where $L_{1}$ and $L_{2}$ are the attenuation lengths and $A_{1}$ and $A_{2}$ are attenuation constants.

\subsubsection{Relative Energy Scale}

Stopping muons are muons with tracks confined within the MINOS detectors and have a well defined momentum. The momentum and range of

such events were used to reconstruct the mean energy loss per distance or $\frac{d E}{d x}$ curve [81, 103], as shown in Fig. 3.11 for the FD.

To define the MEU constants, only the portion of the muon track where

$\frac{d E}{d x}$ varies slowly, defined as the track window, was used in order to limit the effect of the uncertainty in the end point of the track on the energy deposition determination. The track window corresponded to $83 \mathrm{~cm}$ of material or 14 perpendicularly traversed planes and started $95 \mathrm{~cm}$ from the end point of the muon track, thereby only employing the response of muons when their energy 
was between 0.5 and $1.1 \mathrm{GeV}$ and discarding the rapid ionization occurring at the end of the track. The MEU constant in each detector roughly equals the median detector response to a $1 \mathrm{GeV}$ muon perpendicularly traversing one scintillator plane and depositing an energy of approximately $2 \mathrm{MeV}$. The detector response in the track window to a single muon is calculated as

$$
\frac{1}{N_{p}} \sum_{i=1}^{N_{p}} \frac{S_{i}}{L_{i}(1+\beta(L-1))},
$$

where $N_{p}$ is the number of planes in the track window, $S_{i}$ is the total response measured in plane $i$ of the track window, and $L_{i}$ is the muon path length through that plane. A track length correction $\beta=0.01$ reduces the impact of the radiative component of the muon energy loss.

The effectiveness of the calibration procedure, as discussed in the Section 3.5.1, was determined by evaluating the degree to which the calibrated response of each detector is uniform in space and time. The raw and calibrated detector responses as a function of position are shown in Figure 3.12 for the MINOS and MINOS+ eras. The $30 \%$ variation in response that was observed across the face of each detector is effectively removed by the calibration chain. Figure 3.13 shows the calibrated response of the detectors as a function of time during the MINOS+ era. A similar flat behavior is observed during the MINOS era. 

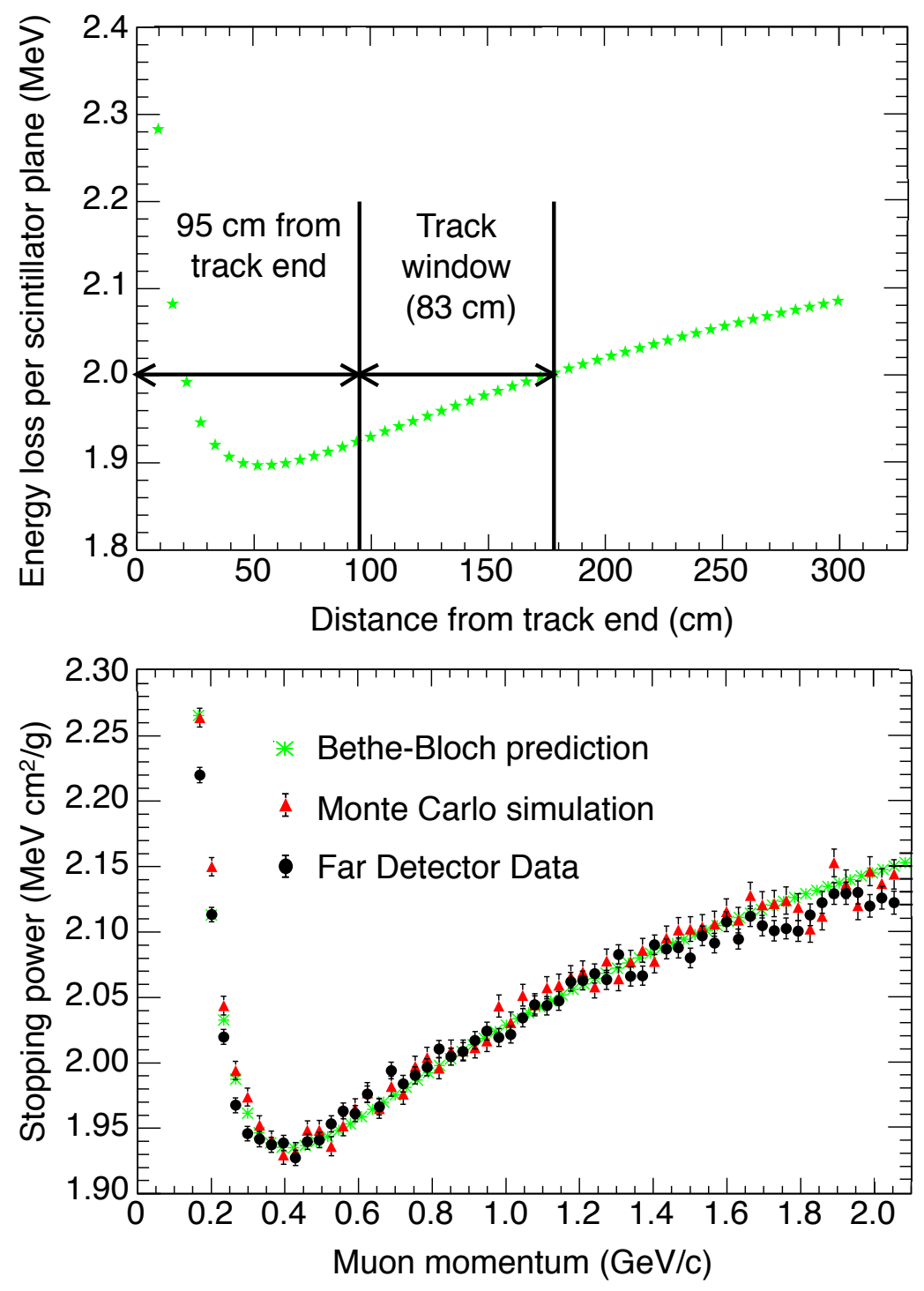

Figure 3.11: Muon energy loss in MINOS polystyrene scintillator. (Top) Energy loss per scintillator plane along the muon tracks [103]. (Bottom) Stopping power or mean energy loss per distance of stopping muons in the Far Detector as a function of momentum [103]. 


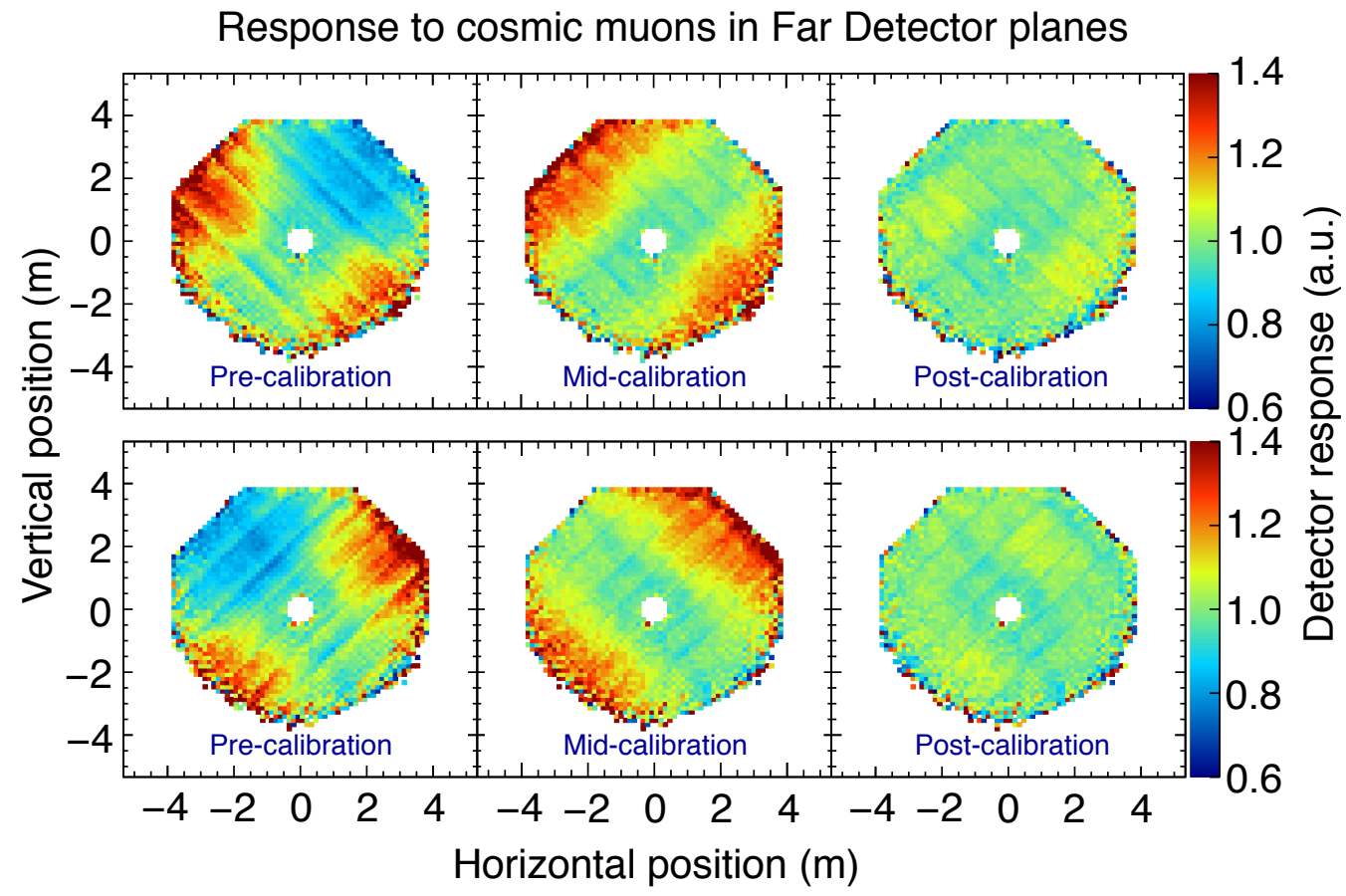

Response to cosmic muons in Near Detector planes

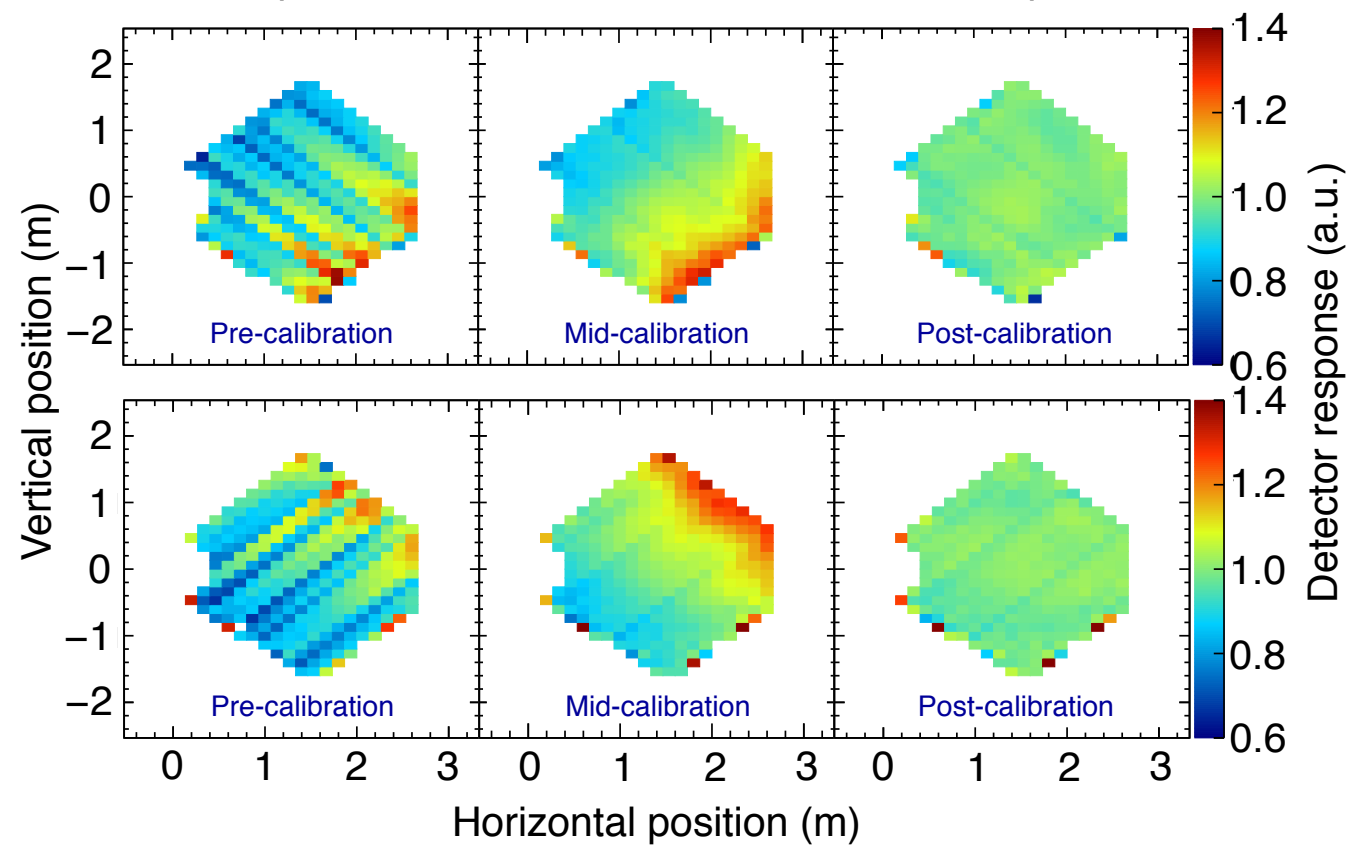

Figure 3.12: The effect of the calibration procedure on the detector response to cosmic muons for Far Detector (top panels) and Near Detector (bottom panels) planes as a function of the vertical and horizontal position during the MINOS and MINOS+ eras. Mid-calibration refers to the stage between strip-to-strip and attenuation calibration. 

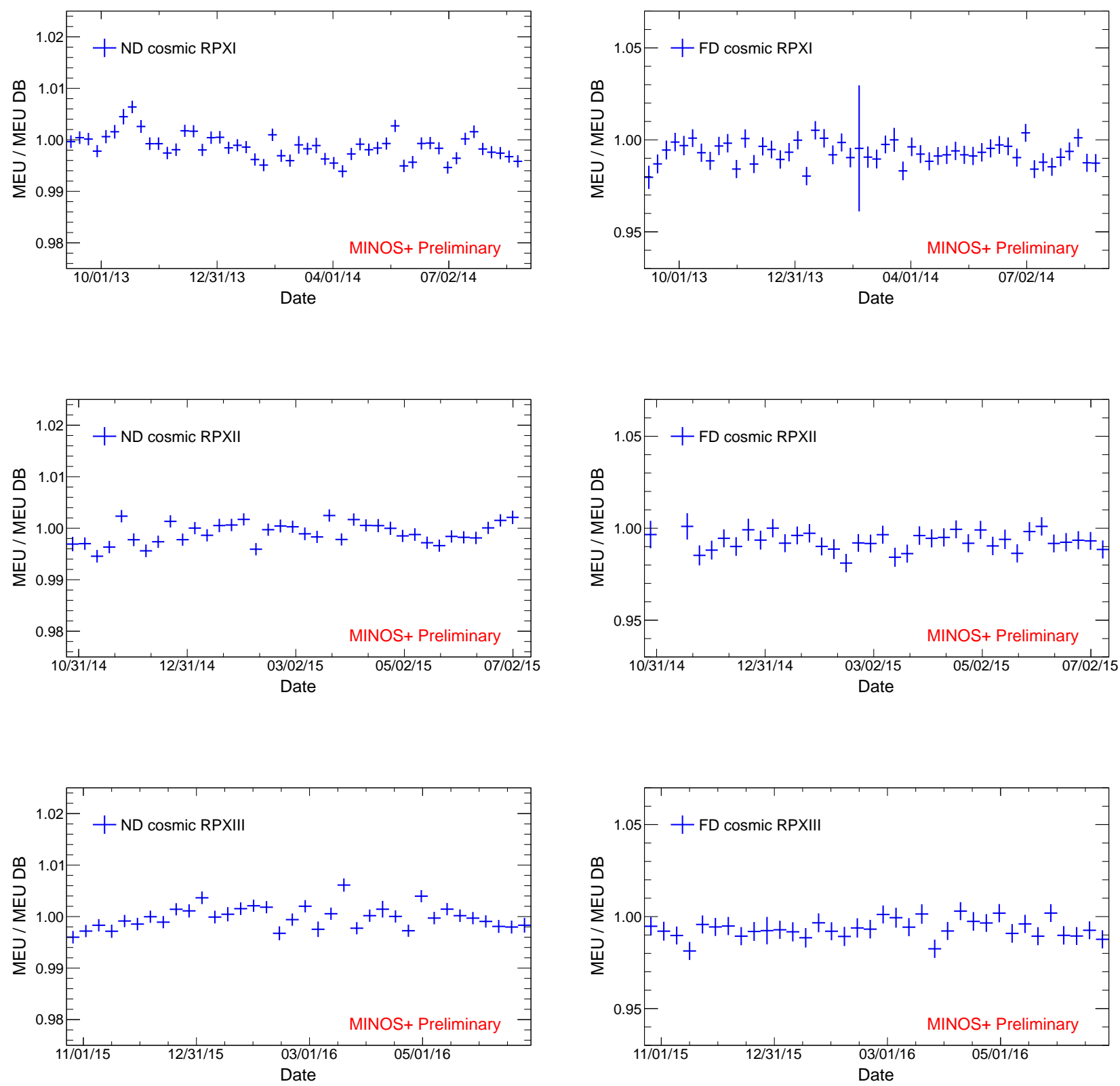

Figure 3.13: The calibrated response of the detectors as a function of time for the ND (left) and FD (right) during the three years of MINOS+ operation, run periods (RP) XI through XIII. The ratio of MEU to MEU DB shows the fractional change in MEU w.r.t. the constant values used throughout the MINOS and MINOS+ eras. 


\subsection{Simulating the MINOS and MINOS+ Experiments}

This section summarizes the NuMI beam and MINOS detector simulation and event reconstruction.

\subsubsection{NuMI Beam Simulation}

The production of the hadron parents of the neutrinos from proton interactions with the NuMI target is simulated with the FLUGG software package [104, 105]. FLUGG employs the GEANT4 software package [106] to provide a simulation of the NuMI target geometry and beamline configuration, and the FLUKA software package [107] to simulate the hadron production at the target.

\subsubsection{SKZP Weights}

To optimize agreement between data and $\mathrm{MC}$ simulation in the ND, a set of weights, called SKZP weights [108], are applied to the spectrum of the simulated neutrino parents. Figure 3.14 shows the effect of the SKZP weights on the ND reconstructed energy spectrum. It should be noted that the SKZP weights are not used for the LED analyses presented in this dissertation. The SKZP weights are obtained under the assumption that there are no neutrino oscillations along the ND baseline, between the neutrino production point and the ND. While this is a good assumption in the three-flavor paradigm, Fig. 2.9 shows that this is not valid in the LED model. However, a subset of the SKZP

weights, the APS weights [109], are applied to the FLUGG flux prediction 

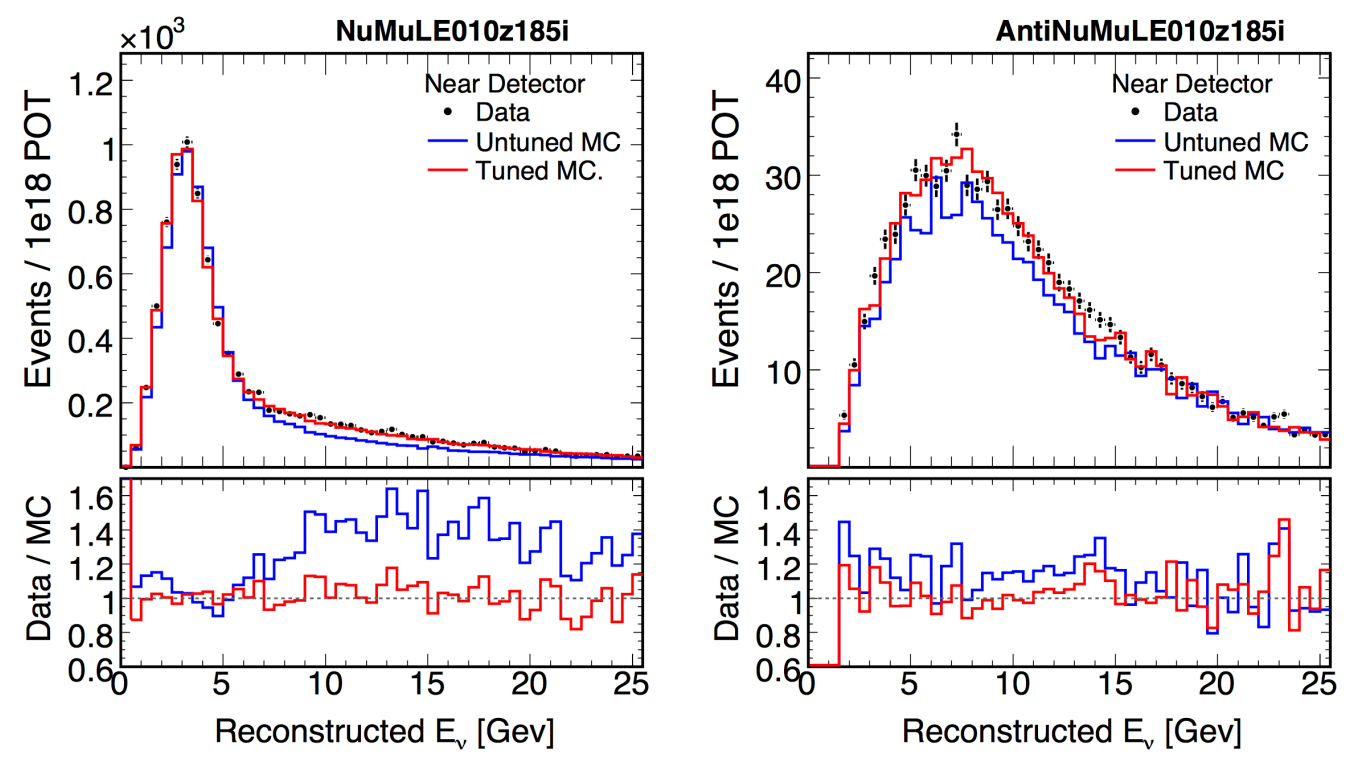

Figure 3.14: The reconstructed neutrino (left) and antineutrino (right) energy spectra at the ND from the first MINOS run period for data (black points) and MC simulation, before (blue line) and after (red line) applying the SKZP weights. This figure is taken from Ref. [110].

in the Far-over-Near LED analysis, discussed in Chapter 5, to correct the flux simulation based on monitoring of NuMI beam conditions. Furthermore, an appropriate systematic uncertainty is assigned to the hadron production simulated using FLUKA, as shown in Section 5.2.10.

\subsubsection{PPFX Weights}

For the two-detector analysis presented in Chapter 6, the PPFX software package [91] is employed to generate correction weights for the FLUGG neutrino flux prediction. This package provides hadron production corrections based on data from hadron production experiments and does not employ 
NuMI data. The method to derive the correction weights is discussed in Section 6.1.1 and also provides a robust estimate of the systematic uncertainty in the hadron production, shown in Section 6.2.8.

\subsubsection{MINOS Detector Simulation}

The neutrino interactions in the MINOS detectors, discussed in Section 3.3, are simulated with NEUGEN [111]. The interaction product particles simulated by NEUGEN are passed to the GMINOS framework [112], which propagates the particles through the detector geometry, taking into account the relevant physics processes using the GCALOR software package [113]. The energy deposits from the propagating particles are stored on a strip-by-strip basis and translated into scintillator light signal using the $\mathrm{C}++$ program $\mathrm{Pho-}$ ton Transport. Taking into account the detector response, electronics noise, and non-linearity of the detector components, Photon Transport provides the number of PE at the PMT cathodes. The $\mathrm{C}++$ program DetSim then propagates the readout from the electronics to the MINOS reconstruction software.

\subsection{Event Reconstruction}

The goal of the reconstruction is to identify the type and energy of the neutrino interactions in the MINOS detectors from the timing and topology of energy depositions in the detectors, as illustrated in Fig. 3.5 and 3.6.

Scintillation light from ionizing particles passing through the MINOS detectors was transported by the WLS and clear fibers to the PMT cathodes 
and converted into electric charge read out by electronics. The registered raw pulse height was digitized and is corrected by the calibration chain in Formula (3.6). A pulse height or hit is associated with a timestamp and a list of possible scintillator strips because of the multiplexing of strips onto the PMTs in the FD, as mentioned in Section 3.2.

A Kalman filter $[114,115]$ algorithm is used to reconstruct tracks and provides an estimate of the charge-to-momentum ratio of the muon track, $q / p$. The muon energy is determined from the track range for tracks confined within the detector and from the track curvature for tracks exciting the detector. A clustering algorithm groups the remaining hits to into hadronic showers. At reconstruction the shower energy is estimated by the sum of all the clustered hit deposits, referred to as the calorimetric shower energy.

An event builder is employed to group associated tracks and showers into events and assign an interaction vertex, usually from the first hit of the primary track in the event [116].

The total reconstructed energy of a neutrino event is then given by

$$
E_{v}=E_{\text {track }}+E_{\text {hadronic }}
$$

where a good hadronic shower energy resolution is important to allow precision measurements of neutrino oscillation parameters. A multivariate $k$-nearestneighbor $(k \mathrm{NN})$ algorithm, employing shower topology features in addition to calorimetric shower energy, was developed for MINOS [117] and optimized for MINOS+ [45] and leads to a better energy resolution than that obtained 
using the calorimetric energy. For an observed data event, a group of closest matching $\mathrm{MC}$ events is identified and their average true energy is used as reconstructed energy for the data event. The distance $d$ of a data event to a training event is defined as

$$
d=\sqrt{\sum_{i=1}^{3} \frac{\left(x_{i}-y_{i}\right)^{2}}{\sigma_{i}^{2}}}
$$

where $\mathbf{x}=\left(x_{1}, x_{2}, x_{3}\right)$ and $\mathbf{y}=\left(y_{1}, y_{2}, y_{3}\right)$ are the $k \mathrm{NN}$ shower variable values for the training and data event, respectively, and $\sigma_{i}$ is the standard deviation of the $i^{\text {th }}$ variable distribution. Figure 3.15 shows the distributions of the three variables considered for the MINOS and MINOS $+k \mathrm{NN}$ algorithm:

- the number of planes in the primary shower,

- the calorimetric energy within $1 \mathrm{~m}$ of the track vertex, and

- the calorimetric energy in the first two reconstructed showers when there is more than one shower in the event.

In MINOS, the 400 nearest neighbors are used to estimate the reconstructed shower energy of a data event, while 480 are used in MINOS+.

The energy resolution of a reconstructed event is given by the quadratic sum of the track and shower energy resolutions

$$
\sigma_{E_{v}}=\sigma_{E_{\mathrm{trk}}} \oplus \sigma_{E_{\mathrm{shw}}}
$$

The shower energy resolution is given by [79]

$$
\sigma_{E_{\mathrm{shw}}}=0.257 \mathrm{GeV} \oplus 40.4 \% \sqrt{E_{\mathrm{shw}}} \oplus 8.6 \% E_{\mathrm{shw}}
$$



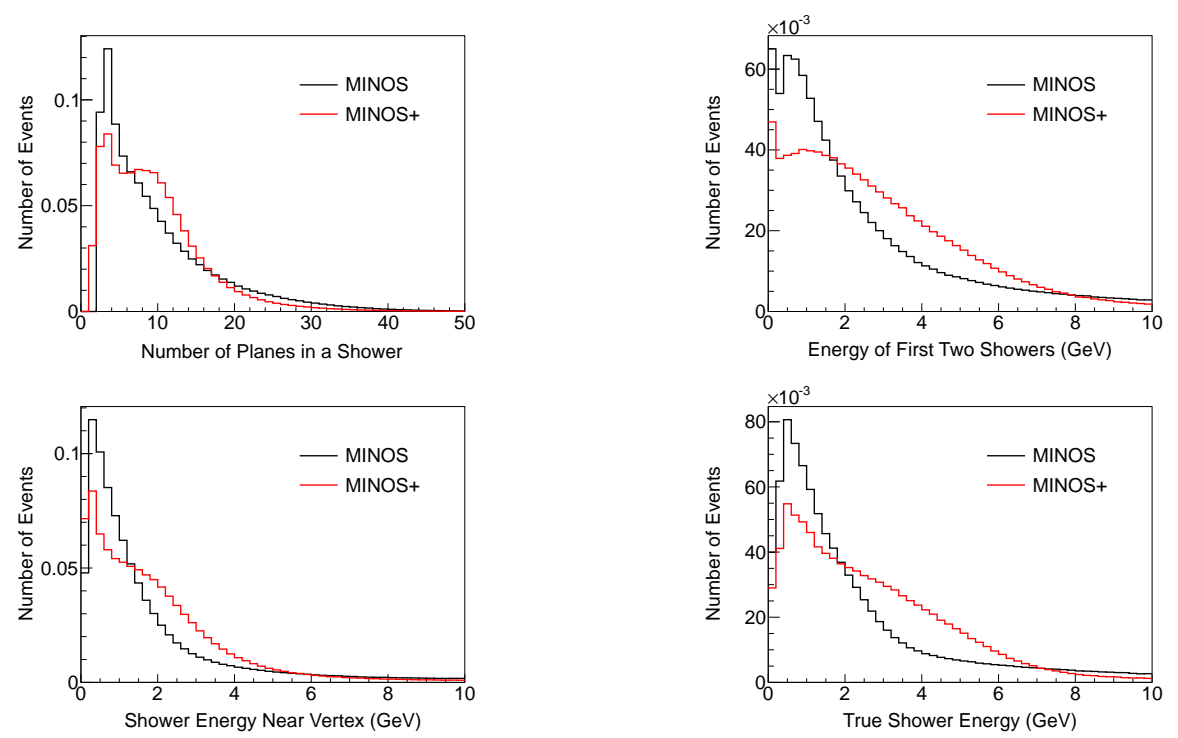

Figure 3.15: Distributions of the three $k \mathrm{NN}$ shower variables (top left, bottom left, and top right) and the true shower energy distributions (bottom right) for MINOS and MINOS+. These figures are taken from Ref. [45].

It should be noted that the calorimetric shower energy resolution obtained from CalDet is given by

$$
\sigma_{E_{\text {cal }}}=56 \% \sqrt{E_{\text {cal }}} \oplus 2 \% E_{\text {cal }} .
$$

The track energy resolution is given by [79]

$$
\sigma_{E_{\mathrm{trk}}}=5.1 \% \sqrt{E_{\mathrm{trk}}} \oplus 6.9 \% E_{\mathrm{trk}}
$$

for events where the muon energy is determined from the track range, and by

$$
\sigma_{E_{\mathrm{trk}}}=p^{2} \sigma_{q / p} \oplus 1.34 \sqrt{p^{2} \sigma_{q / p}}
$$

for events where the muon energy is determined from the track curvature, with $\sigma_{q / p}$ an estimate of the error in $q / p$ provided by the Kalman filter algorithm. 

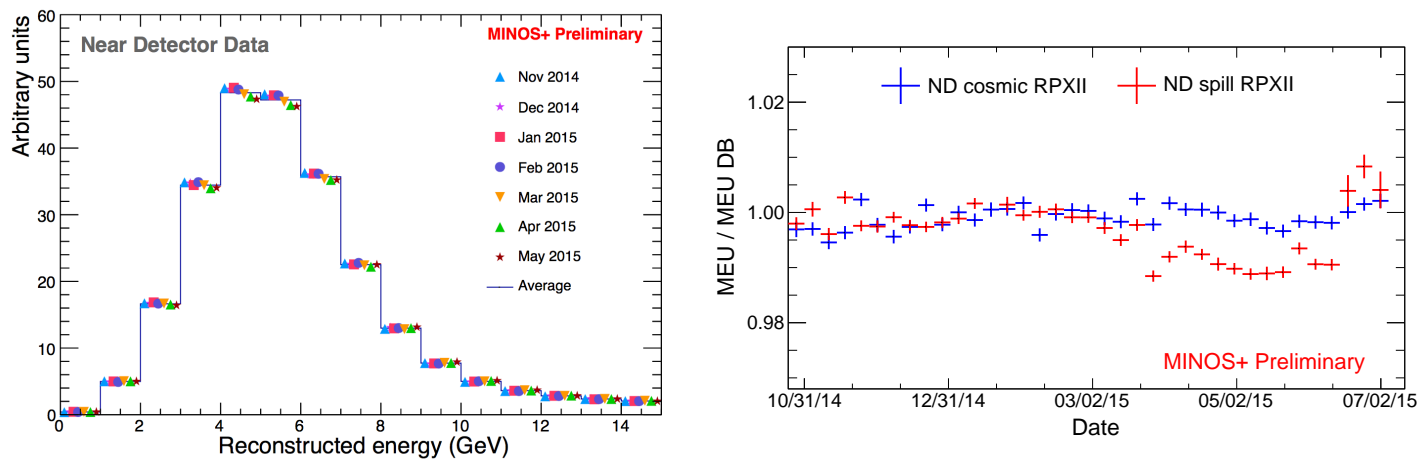

Figure 3.16: The MINOS+ intensity effect. (Left) The ND CC reconstructed energy spectrum for the second year of MINOS+ data taking. A dip is observed in the peak bins for the months March through May 2015. This figure is taken from Ref. [46]. (Right) The intensity effect is observed as a drop in the calibrated response of the ND as a function of time when using stopping muons originating from NuMI neutrino events (red points) to calculate the MEU value.

\subsubsection{Intensity Effect}

It was discovered in March 2015 that a deficit of events was present in the peak of the MINOS+ ND CC energy spectrum once the NuMI beam had switched to a higher intensity running mode, as shown in Fig. 3.16. The effect was shown to be due to the merging of events during event reconstruction, resulting from doubling the number of interactions in the detector [118]. Studies show that for the first two years of MINOS+ data collection, the intensity effect is linear [119]. This allows the intensity effect to be modeled by reweighting the $\mathrm{MC}$ samples according to the actual NuMI beam intensities. A much smaller intensity effect for the MINOS ND energy spectra is discussed in detail in Ref. [46]. 


\section{Chapter 4}

\section{Event Selection}

This chapter describes the event selection procedures employed for the MINOS and MINOS+ data used in the Far-over-Near and two-detector LED analyses, discussed in Chapter 5 and Chapter 6, respectively.

The same event selection requirements are used for MINOS and MINOS+. The event selection for the MINOS sample is discussed in detail in Ref. [46], while Ref. [45] discusses the event selection for the first year of MINOS+ operation. The second year of the MINOS+ sample is also used in this dissertation and the corresponding event selection is included in this chapter.

The event selection procedure starts with the NC event selection using the full set of reconstructed events. Events that are not selected by the NC selector are subsequently used as input for the CC event selection, thereby ensuring that no double counting of events occurs between both selectors.

It should be noted that no charge separation is applied in the event selection procedures employed for this dissertation, such that both $v_{\mu}$ and $\bar{v}_{\mu}$ events are used, thereby implicitly assuming CPT symmetry.

A preselection procedure is discussed in the first section of this chapter, followed by a discussion of the $\mathrm{NC}$ and $\mathrm{CC}$ event selection. The final two 
sections assess the performance of the event selection procedures and show the selected energy spectra, respectively.

\subsection{Preselection}

An initial selection procedure, or preselection, is applied to all events that are provided by the event reconstruction, discussed in Section 3.7, to remove events that are reconstructed while the NuMI beam or MINOS detectors were not operating in normal conditions or for which timing and topology conditions are not consistent with NuMI beam neutrino events.

The preselection procedure removes events in both the ND and FD that are reconstructed with

- a bad NuMI beam quality, abnormal operating conditions for the magnetic coil and/or MINOS detectors;

- events for which the Kalman filter failed to reconstructed the muon track;

- events reconstructed during operation of the LI system;

- events reconstructed outside the NuMI beam spill window of $10 \mu \mathrm{s}$;

- events for which the angle $\theta$ between the muon track and the NuMI beam axis has $\cos \theta<0.6$, thereby removing events that are induced by cosmic muons interacting within the detector.

Because of the high interaction rates, multiple events overlap both in space and time in the ND. This causes reconstruction failures at low energies 
that often result in small shower-like topologies that could be misidentified as $\mathrm{NC}$ events. A set of preselection requirements is employed to reduce the number of poorly reconstructed ND events, which are defined as events with reconstructed energy less than $30 \%$ of the true energy. Events in the ND are removed when

- the maximum number of consecutive scintillator planes with energy deposits is less than three;

- the pulse height fraction, or the ratio of pulse height in the event to the total pulse height in the group of hits from which the event is reconstructed, is smaller than $50 \%$.

Figure 4.1 shows the two preselection requirements to remove poorly reconstructed events in the ND for the first two years of MINOS+ operation and with the MINOS sample included.

Similarly, a set of preselection requirements are imposed on the FD reconstructed events to reduce electronic noise, the cosmic muon background, and the fake events induced by the LI system, as discussed in more detail in Ref. [46].

\subsection{Neutral Current Event Selection}

After the preselection, the NC event selection is applied. Selection requirements are imposed based on the position of the event vertex in the detector and the topology of the event. 

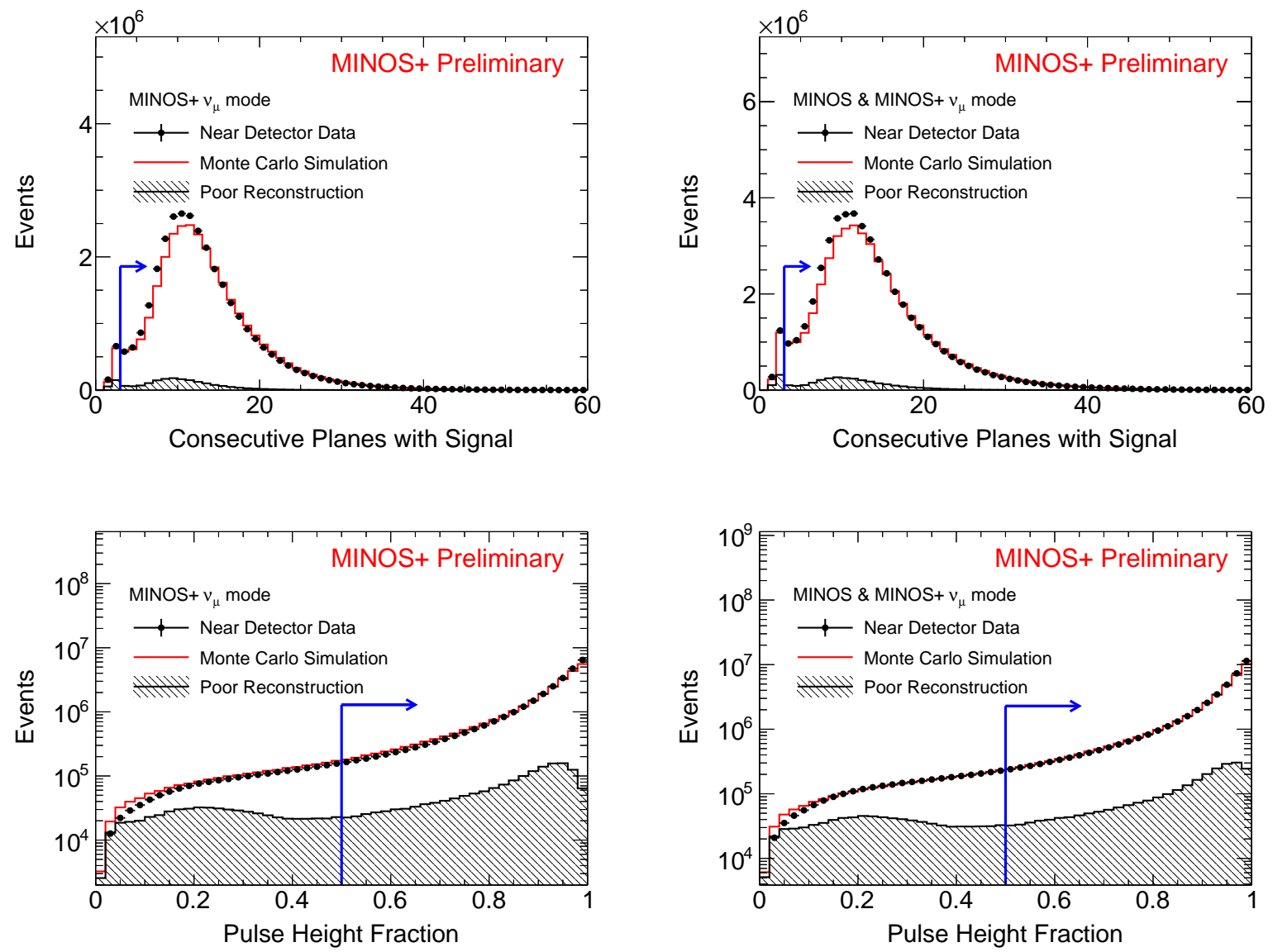

Figure 4.1: Preselection requirements to remove poorly reconstructed events in the ND. The maximum number of consecutive planes with energy deposits is required to be larger than three (top row), and the pulse height fraction needs to be larger than $50 \%$ (bottom row). The event selection is shown for both the first two years of MINOS+ only (left column) and with the MINOS sample added (right column). Data (black points) are compared to unoscillated MC simulation (red line) and the fraction of poorly reconstructed events is shown (hatched histogram). The blue arrow indicates the region of the plot for which events are accepted by the ND preselection. These figures are taken from Ref. [120]. 


\subsubsection{Acceptance Selection}

A fiducial volume is defined in the ND and FD to allow the removal of events for which part of the energy deposition occurs outside of the detector [121]. Events can either have a vertex inside the detector but with an energy deposition that is not fully contained in the detector, or a vertex outside the detector but with part of the energy deposition contained within the detector. In the former case the calorimetric shower energy does not provide an accurate estimate of the reconstructed neutrino energy, while in the latter case the reconstruction algorithm might incorrectly assign a vertex within the detector to the event.

The fiducial volume in the ND is defined such that event vertices are $0.5 \mathrm{~m}$ away from the edges of the $\mathrm{U}$ and $\mathrm{V}$ scintillator planes. Along the NuMI beam axis, event vertices are required to have a $Z$ coordinate between $1.7 \mathrm{~m}$ and $4.7368 \mathrm{~m}$, where $Z=0 \mathrm{~m}$ corresponds to the first scintillator plane. Figure 4.2 shows the distributions of the event vertex coordinates for data and $\mathrm{MC}$ events in the ND.

At the FD, the fiducial volume is defined such that event vertices are $0.4 \mathrm{~m}$ away from the edges of the scintillator planes and $0.6 \mathrm{~m}$ away from the coil hole center. Along the NuMI beam axis, event vertices are required to have $0.21 \mathrm{~m}<Z<13.72 \mathrm{~m}$ for the first FD module and $16.12 \mathrm{~m}<Z<28.96 \mathrm{~m}$ for the second FD module. Figure 4.3 shows the distributions of the event vertex coordinates for data and MC events in the FD, where standard three-flavor oscillation probabilities are applied to the MC sample. 

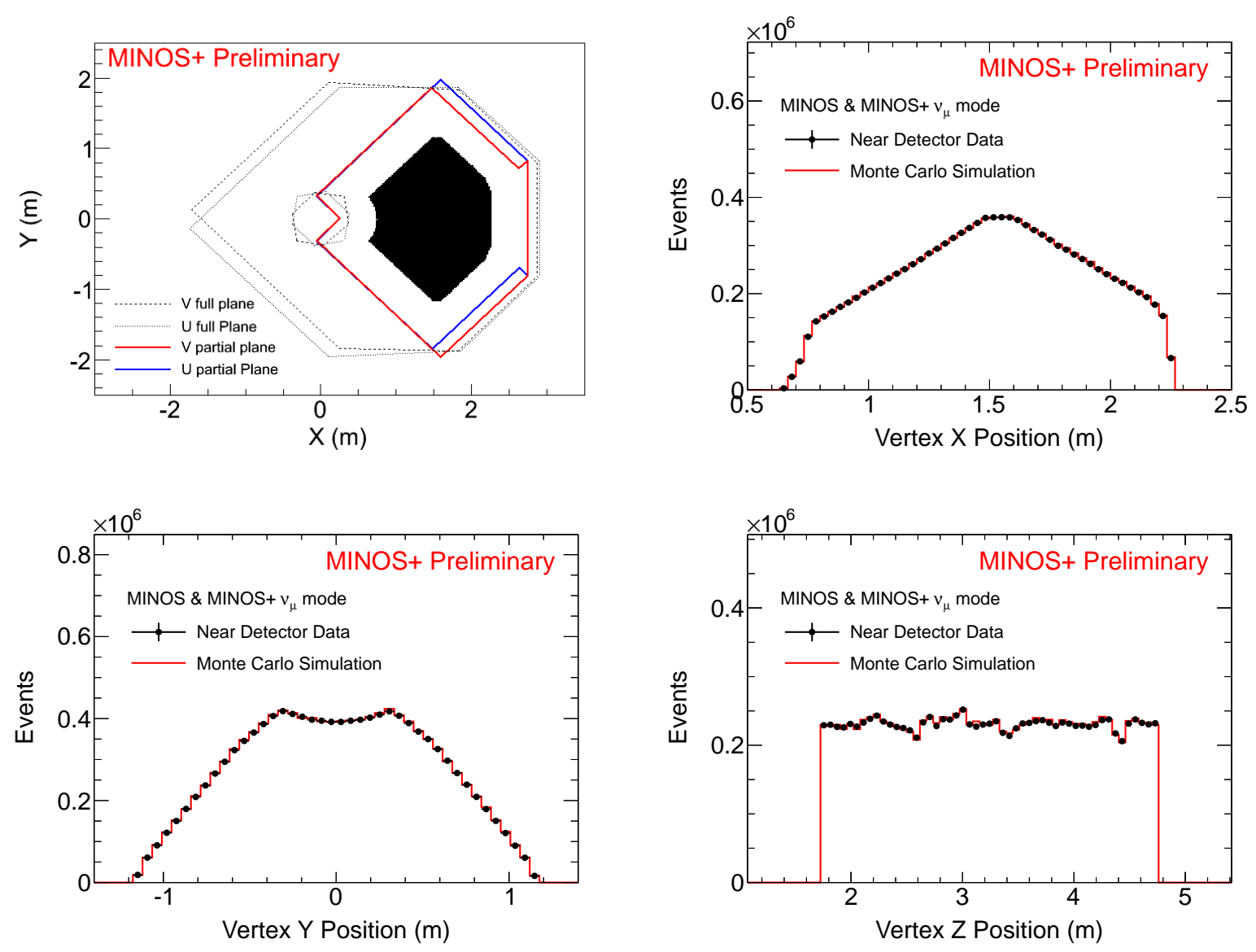

Figure 4.2: Distribution of the event vertex coordinates in the ND. The $X Y$ view of the $\mathrm{NC}$ event vertices (top left), with the solid black shape representing the accepted region. The $X$ coordinate distribution (top right), the $Y$ coordinate distribution (bottom left), and the $Z$ coordinate distribution (bottom right) of ND data and MC events. These figures are taken from Ref. [120]. 

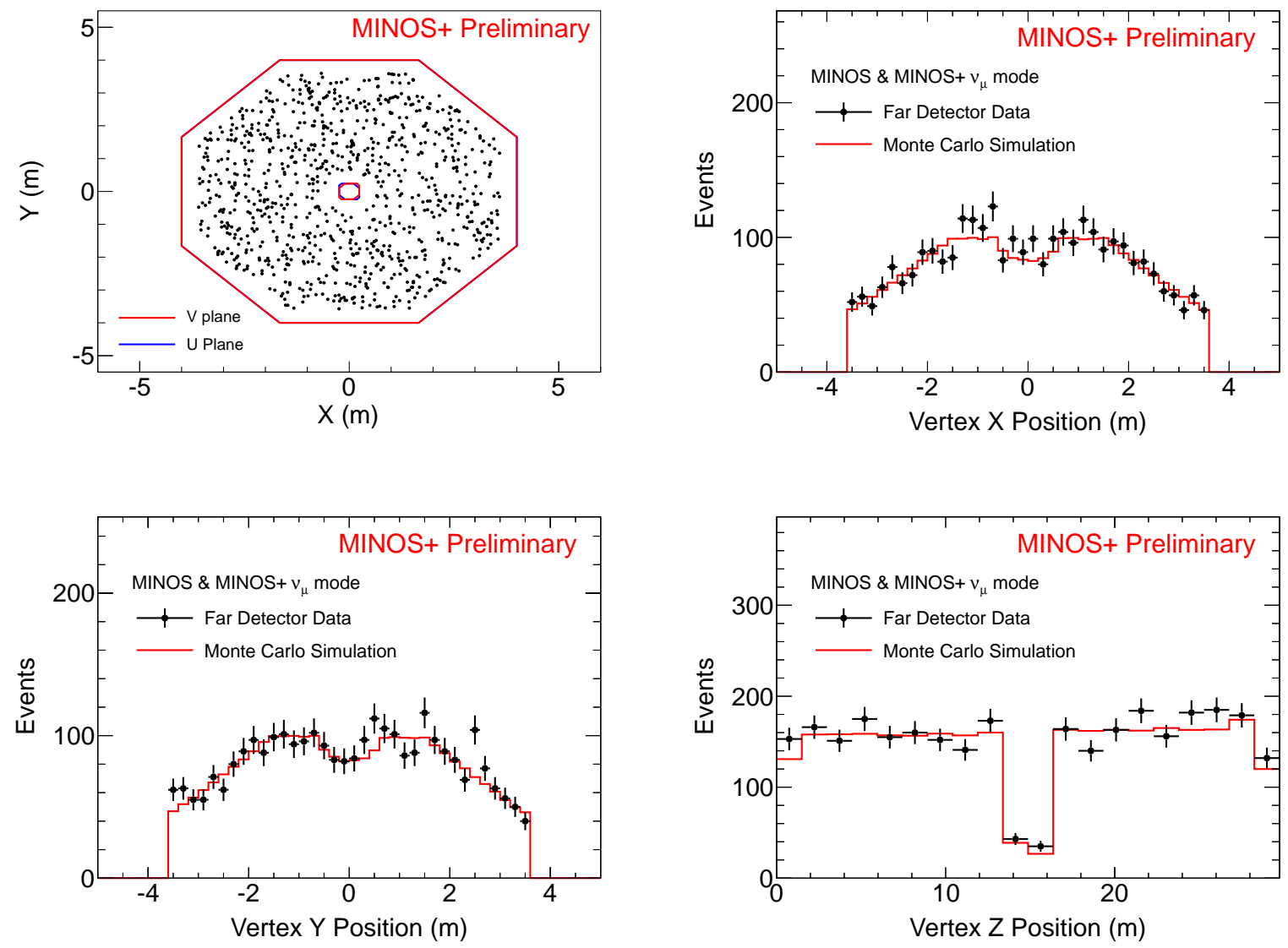

Figure 4.3: Distribution of the event vertex coordinates in the FD. The $X Y$ view of the $\mathrm{NC}$ event vertices (top left). The $X$ coordinate distribution (top right), the $Y$ coordinate distribution (bottom left), and the $Z$ coordinate distribution (bottom right) of FD data and MC events, assuming three-flavor oscillation. The drop in events around $Z=15 \mathrm{~m}$ corresponds to the gap between the two FD modules. These figures are taken from Ref. [120]. 


\subsubsection{Topology Requirements}

To reduce the background of $\mathrm{CC}$ events in the $\mathrm{NC}$ selected sample, two additional topology selection requirements are imposed in the ND and FD. Events are removed when

- the energy deposition occurs in more than 47 consecutive planes,

- the event has a reconstructed track that extends more than 5 planes beyond the end of the shower.

Figure 4.4 shows the topology selection requirements in the ND for the first two years of MINOS+ operation and with the addition of the MINOS sample. Figure 4.5 shows the same for the FD, assuming standard three-flavor oscillation.

\subsection{Charged Current Event Selection}

Reconstructed events that do not fulfill the NC selection requirements are used in the CC selection procedure. Selection requirements are imposed based on the position of the event vertex in the detector and a $k \mathrm{NN}$ algorithm is applied to separate CC events from NC events. Events that do not fulfill the $\mathrm{CC}$ selection requirements are not employed in the analyses presented in this dissertation. 

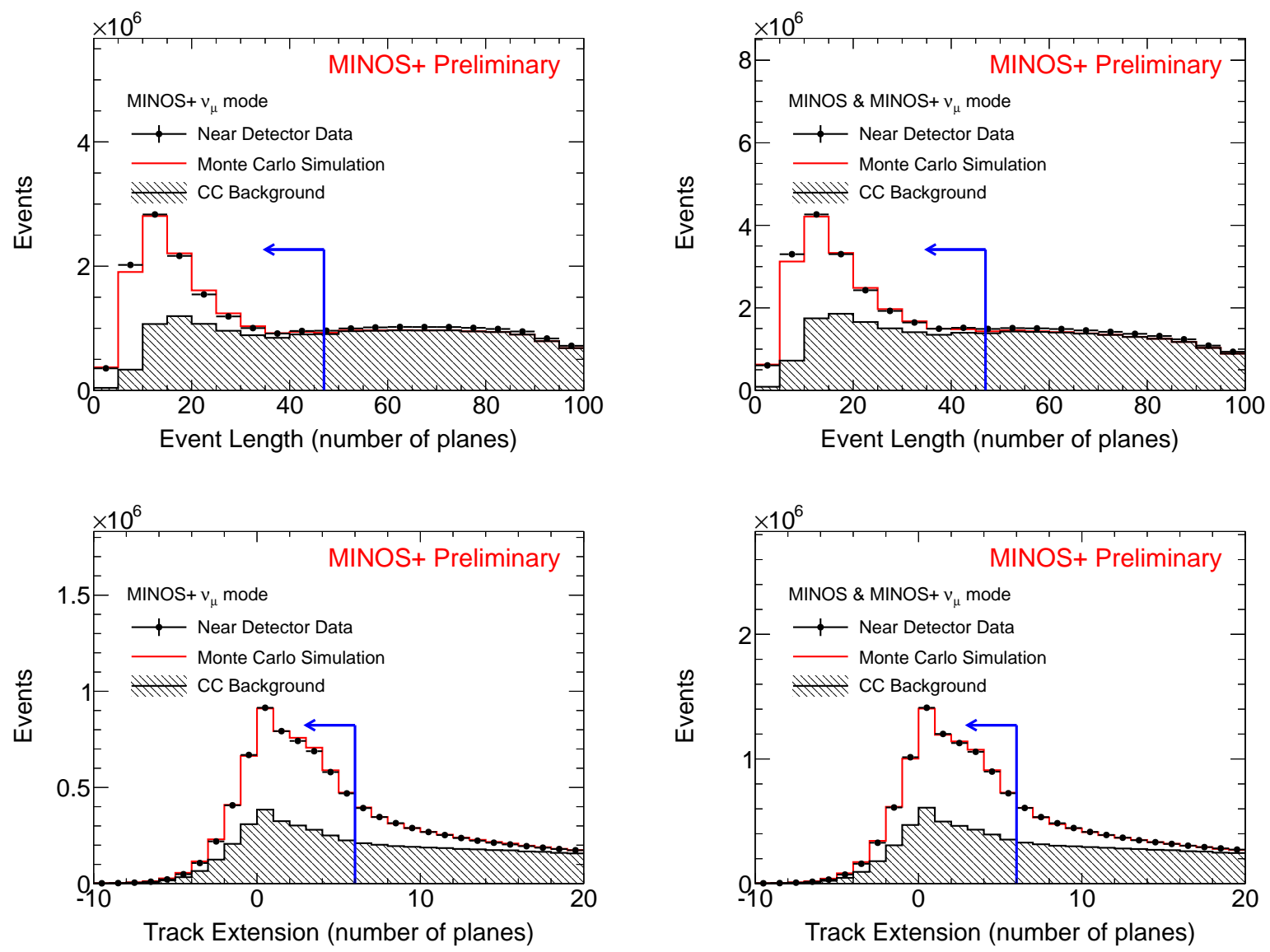

Figure 4.4: Topology selection requirements to remove CC background events in the ND. The energy deposition cannot occur in more than 47 consecutive planes (top row), and the event cannot contain a track that extends more than 5 planes beyond the end of the shower (bottom row). The event selection is shown for both the first two years of MINOS+ only (left column) and with the MINOS sample added (right column). Data (black points) are compared to unoscillated MC simulation (red line) and the fraction of poorly reconstructed events is shown (hatched histogram). The blue arrow indicates the region of the plot for which events are accepted by the ND preselection. These figures are taken from Ref. [120]. 

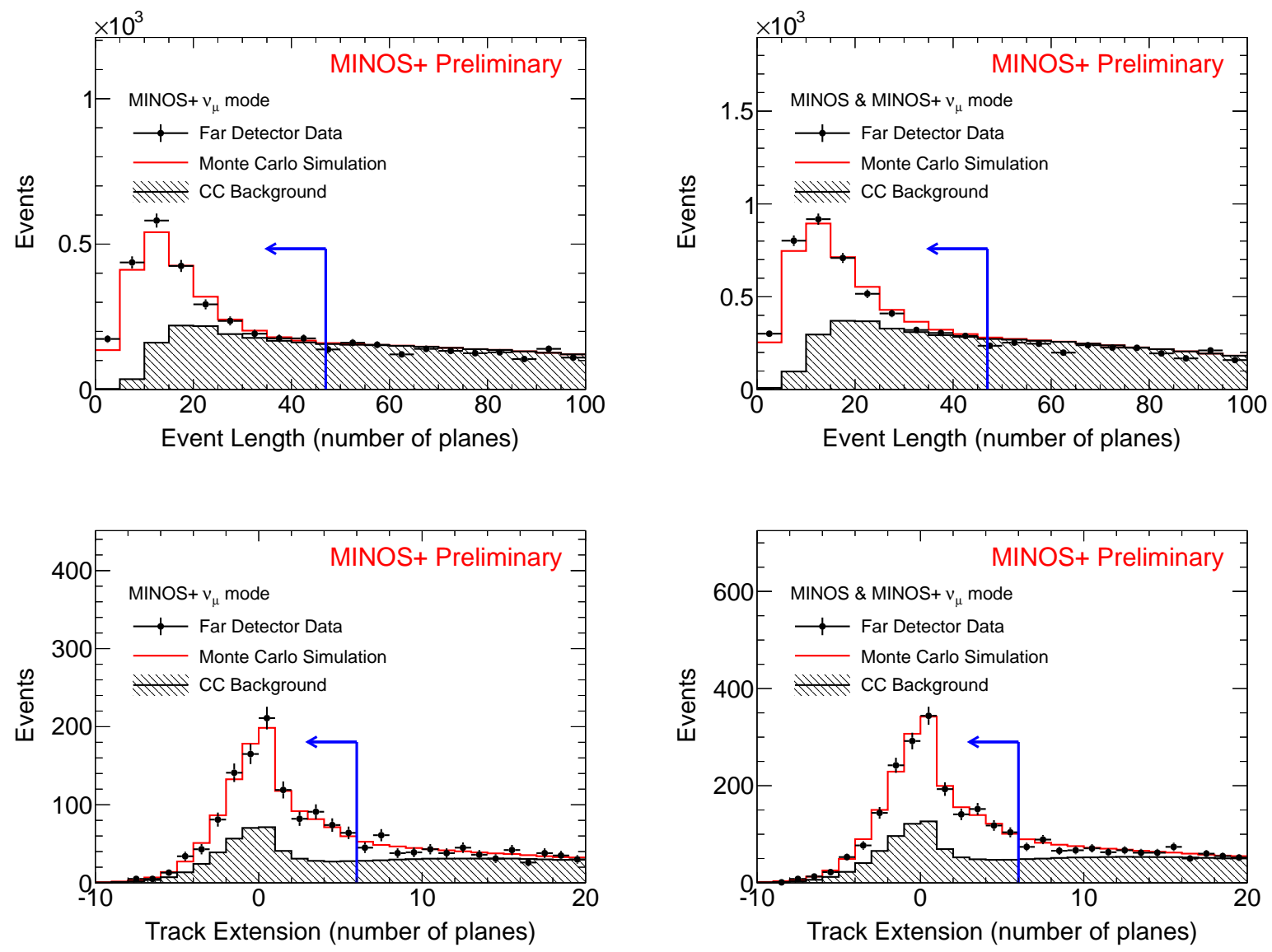

Figure 4.5: Topology selection requirements to remove CC background events in the FD. The energy deposition cannot occur in more than 47 consecutive planes (top row), and the event cannot contain a track that extends more than 5 planes beyond the end of the shower (bottom row). The event selection is shown for both the first two years of MINOS+ only (left column) and with the MINOS sample added (right column). Data (black points) are compared to three-flavor MC simulation (red line) and the fraction of poorly reconstructed events is shown (hatched histogram). The blue arrow indicates the region of the plot for which events are accepted by the FD preselection. These figures are taken from Ref. [120]. 


\subsubsection{Acceptance Selection}

The $X Y$-view of the ND fiducial volume for $\mathrm{CC}$ events is shown in the left panel of Fig. 4.6, with the neutrino beam traveling toward the reader and having a beam center at $X_{0}=1.4828 \mathrm{~m}$ and $Y_{0}=02384 \mathrm{~m}$. The fiducial volume is a cylinder with radius $R=0.8 \mathrm{~m}$ and event vertices have a $Z$ coordinate for which $0.81 \mathrm{~m}<Z<4.08 \mathrm{~m}$, with $Z=0$ corresponding to the front surface of the detector. Because the coil hole is not well modeled in the ND simulation, events with a track within $0.6 \mathrm{~m}$ from the coil hole center and ending in the calorimeter part of the detector are removed from the event sample.

The $X Y$-view of the FD fiducial volume for $\mathrm{CC}$ events is shown in the right panel Fig. 4.6. The fiducial volume is a cylinder for which the radius $R$ satisfies $0.5 \mathrm{~m}<R<3.74 \mathrm{~m}$, where $R=0$ corresponds to the central detector axis and the lower limit accounts for the removal of events near or through the coil hole. Event vertices have a $Z$ coordinate for which $0.81 \mathrm{~m}<Z<4.08 \mathrm{~m}$, with $Z=0$ corresponding to the front surface of the detector. Along the NuMI beam axis, event vertices are required to have $0.49 \mathrm{~m}<Z<14.29 \mathrm{~m}$ for the first FD module and $16.27 \mathrm{~m}<Z<27.98 \mathrm{~m}$ for the second FD module.

\subsubsection{Separating Charged and Neutral Current Events}

As discussed in Chapter 3, a CC event has at least one reconstructed track. A $k \mathrm{NN}$ algorithm is employed to separate CC events with a muon track from NC background events where a track is reconstructed from hits of the hadronic shower. Two particle identification (PID) metrics are used in MINOS 

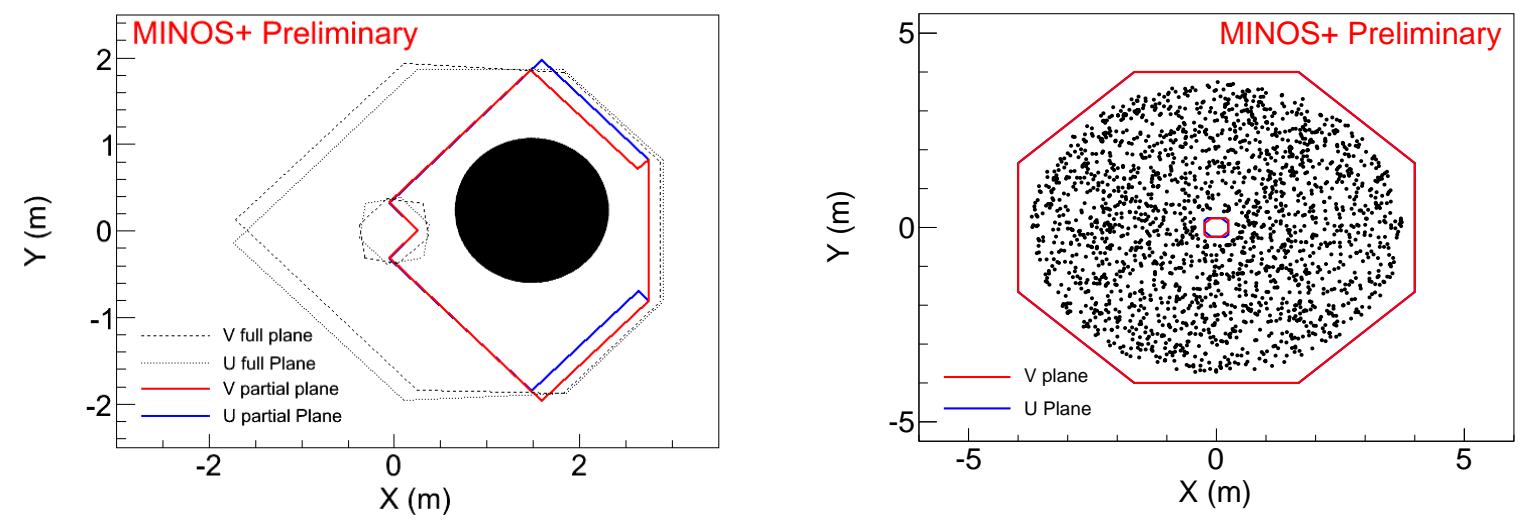

Figure 4.6: The $X Y$-view of the distribution of the CC event vertex coordinates in the ND (left) and FD (right). These figures are taken from Ref. [120].

for this purpose, roID [122] and jmID [123], with the latter being optimized to select more low-energy events to improve the sensitivity of MINOS to the neutrino decay and decoherence models. An event is selected by the CC selection if roID $<0.25$ or jmID $<0.5$. In MINOS+, only roID is employed because of the low event counts at low energies. The optimization of roID in MINOS+ is discussed in detail in Ref. [45].

Similar to the $k \mathrm{NN}$ algorithm to determine the hadronic shower energy, discussed in Section 3.7, a group of closest matching MC events is identified for an observed data event using a distance metric similar to the one defined in Formula (3.12). The fraction of CC events to NC background events is defined as the roID metric

$$
\frac{k_{S}}{k_{S}+k_{B}}=\frac{k_{S}}{k},
$$


where $k_{S}$ and $k_{B}$ are the number of CC events and the number of NC background events, respectively. Four $k \mathrm{NN}$ variables are used to determine the $k$ nearest neighbors,

- the number of scintillator planes in a track,

- the mean pulse height of the track hits,

- the ratio of the mean of low pulse heights to the mean of high pulse heights, and

- the ratio of the total pulse height of the track hits to the total pulse height of the event hits.

Figure 4.7 shows the distributions of these $k \mathrm{NN}$ variables for MINOS+ MC samples. Figure 4.8 shows the distribution of roID with $k=80$ for MINOS+ ND MC samples, with the value of $k$ adopted from MINOS. Events for which roID $>0.3$ are selected as CC events.

\subsection{Event Selection Performance}

The NC and CC event selection procedures are evaluated based on their purity and efficiency. Purity is defined as

$$
\frac{\text { number of selected true signal events }}{\text { total number of selected events }}
$$

and efficiency is defined as

$$
\frac{\text { number of selected true signal events }}{\text { total number of true signal events before selection }} \text {. }
$$



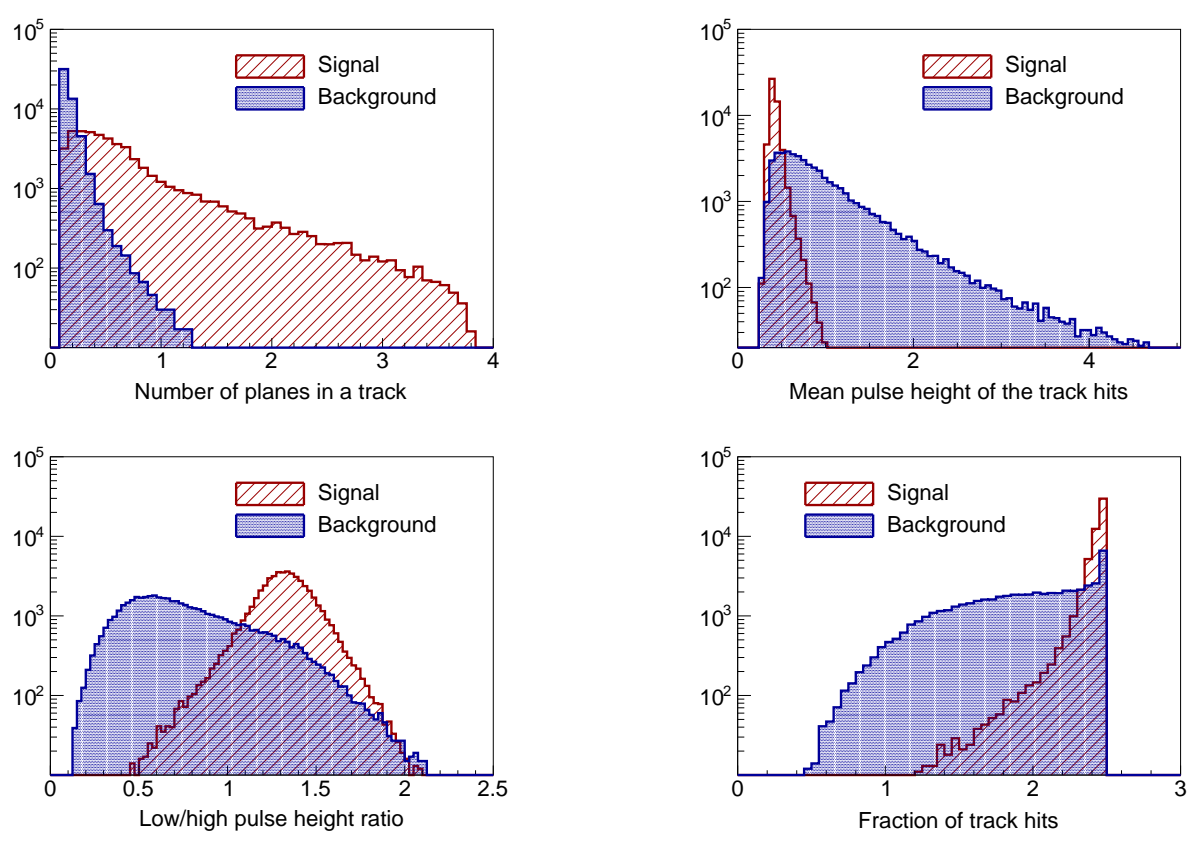

Figure 4.7: Distributions of the four $k \mathrm{NN}$ variables used to separate $\mathrm{CC}$ events from $\mathrm{NC}$ background events. An equal number of $\mathrm{CC}$ events and $\mathrm{NC}$ background events is used for the training set. These figures are taken from Ref. [45].

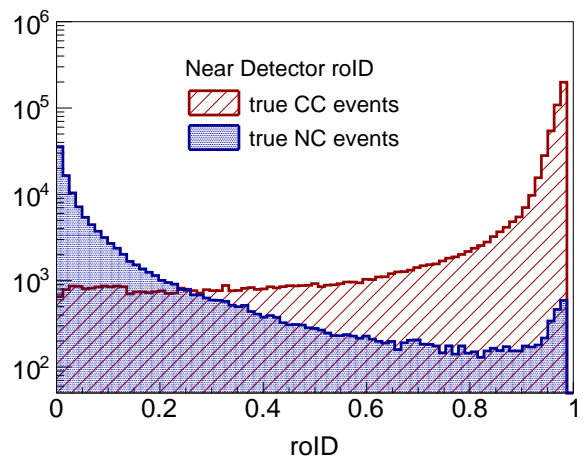

Figure 4.8: Distribution of roID for $k=80$ for the MINOS+ ND MC sample. This figure is taken from Ref. [45]. 
For the NC selection procedure, the denominator of Formula (4.3) is the total number of true $\mathrm{NC}$ events before the two topology selection requirements are imposed. The top row of Fig. 4.9 shows the purity and efficiency as a function of energy for the NC selection in the ND and FD for the first two years of MINOS+ only and with the addition of the MINOS sample.

For the CC selection procedure, the denominator of Formula (4.3) is the total number of true CC events with at least one track and an event vertex inside the fiducial volume. The top row of Fig. 4.9 shows the purity and efficiency as a function of energy for the CC selection. The efficiency of the $\mathrm{CC}$ selection in the ND is low because of the removal of $\mathrm{CC}$ events occurring near the coil hole. This has a negligible impact on the analyses presented in this dissertation because of the high number of events observed in the ND.

\subsection{Selected Samples}

The simulated ND and FD event energy spectra for the CC and NC selected samples for MINOS only, MINOS+ only, and MINOS and MINOS+ are shown in Fig. 4.10, 4.11, and 4.12, respectively. All spectra are obtained assuming three-flavor oscillations. The main backgrounds are NC and CC $v_{\mu}$ events in the CC and NC spectra, respectively. A small $v_{\mathrm{e}}$ background from the NuMI beam is present in both the ND and FD NC selected samples. The FD NC sample contains additional minor backgrounds from $v_{\mathrm{e}}$ and $\boldsymbol{v}_{\tau}$ appearance along the FD baseline. 

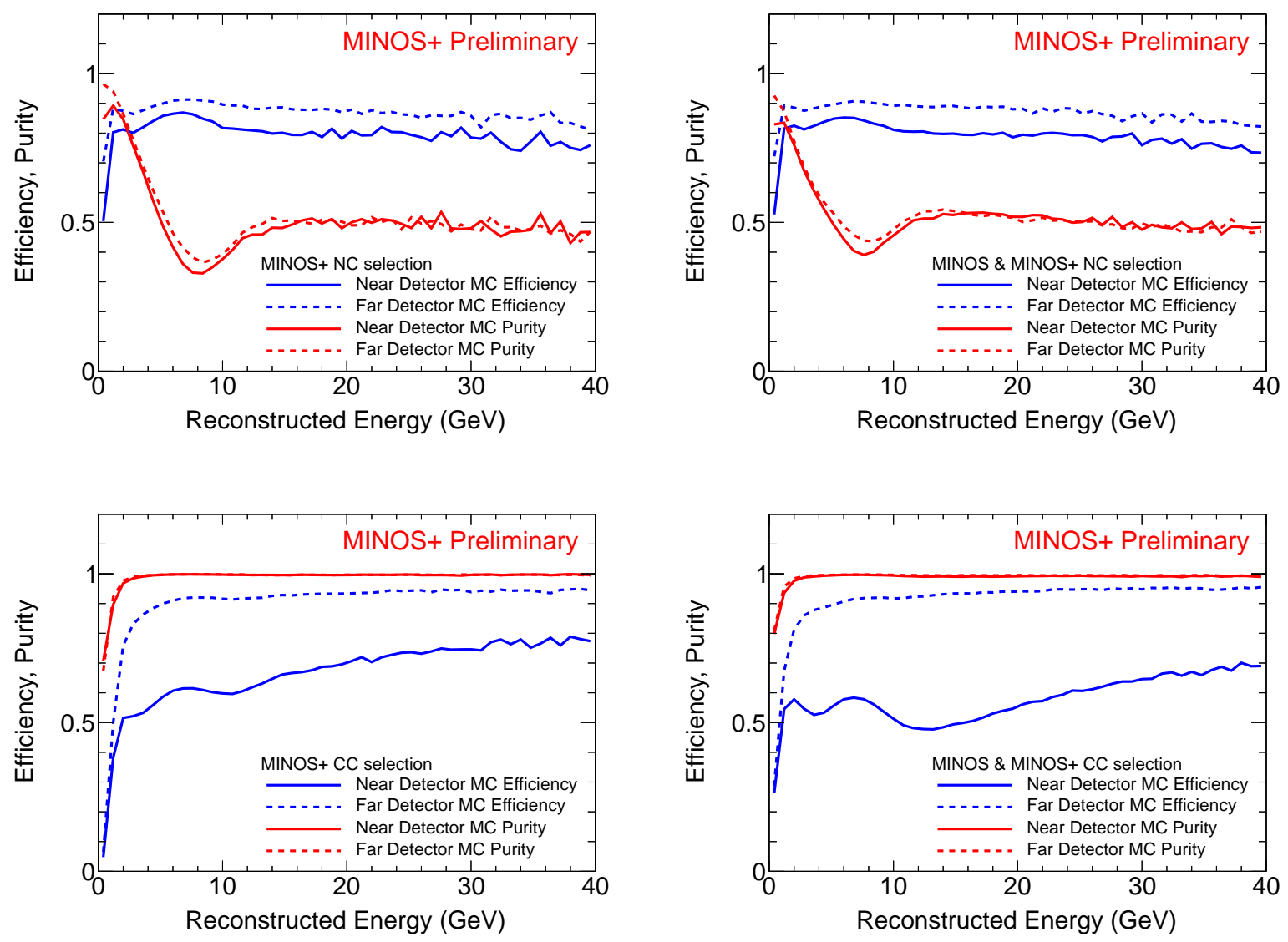

Figure 4.9: Purity and efficiency of the NC (top row) and CC (bottom row) selection procedures in the ND and FD as a function of energy. The purity and efficiency are shown for both the first two years of MINOS+ only (left column) and with the MINOS sample added (right column). These figures are taken from Ref. [120]. 

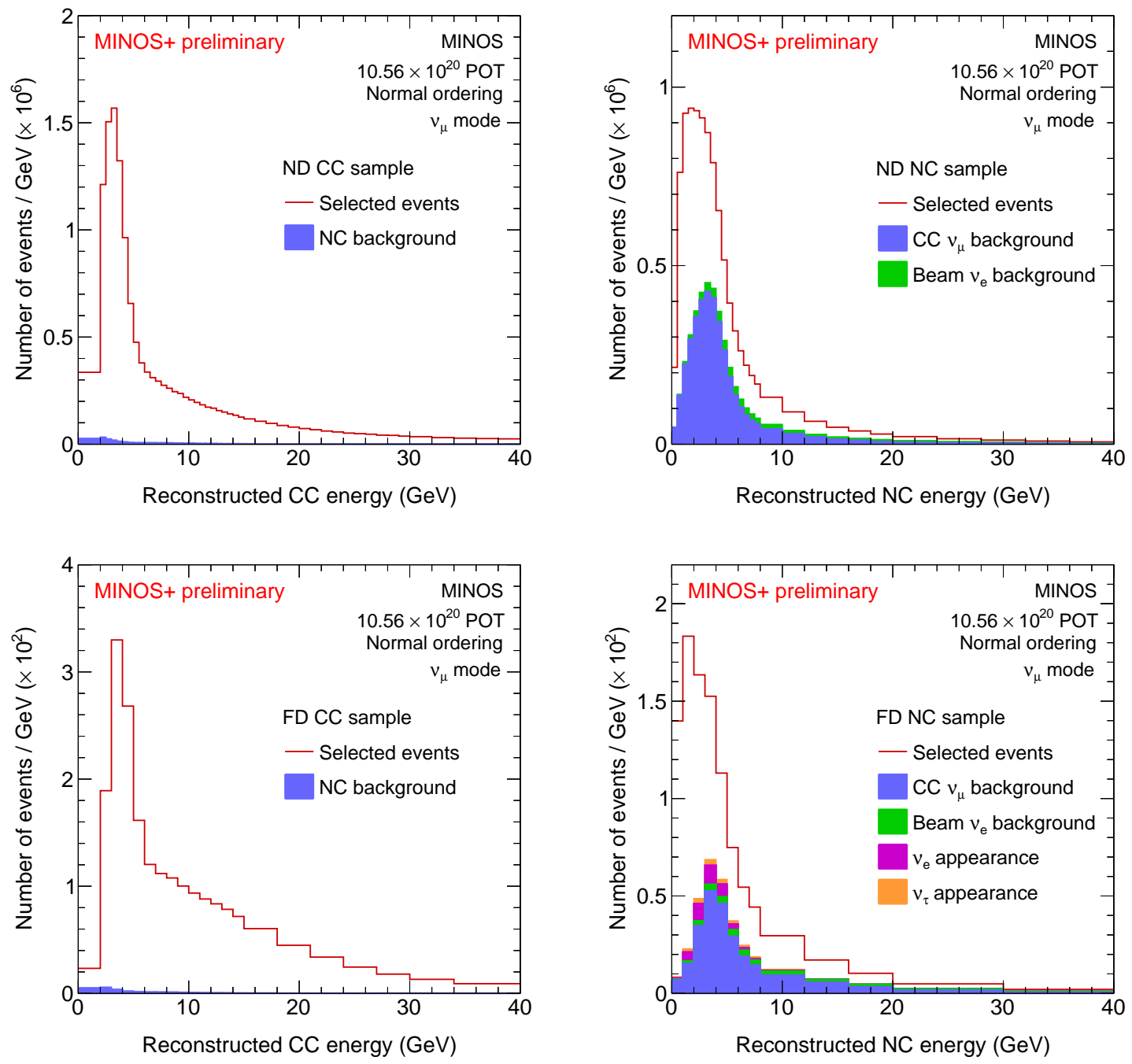

Figure 4.10: Simulated ND (top row) and FD (bottom row) event energy spectra for the CC (left column) and NC (right column) selected samples for MINOS only. The spectra are obtained assuming three-flavor oscillations. The main backgrounds are $\mathrm{NC}$ and $\mathrm{CC} \nu_{\mu}$ events for the $\mathrm{CC}$ and $\mathrm{NC}$ samples, respectively. 

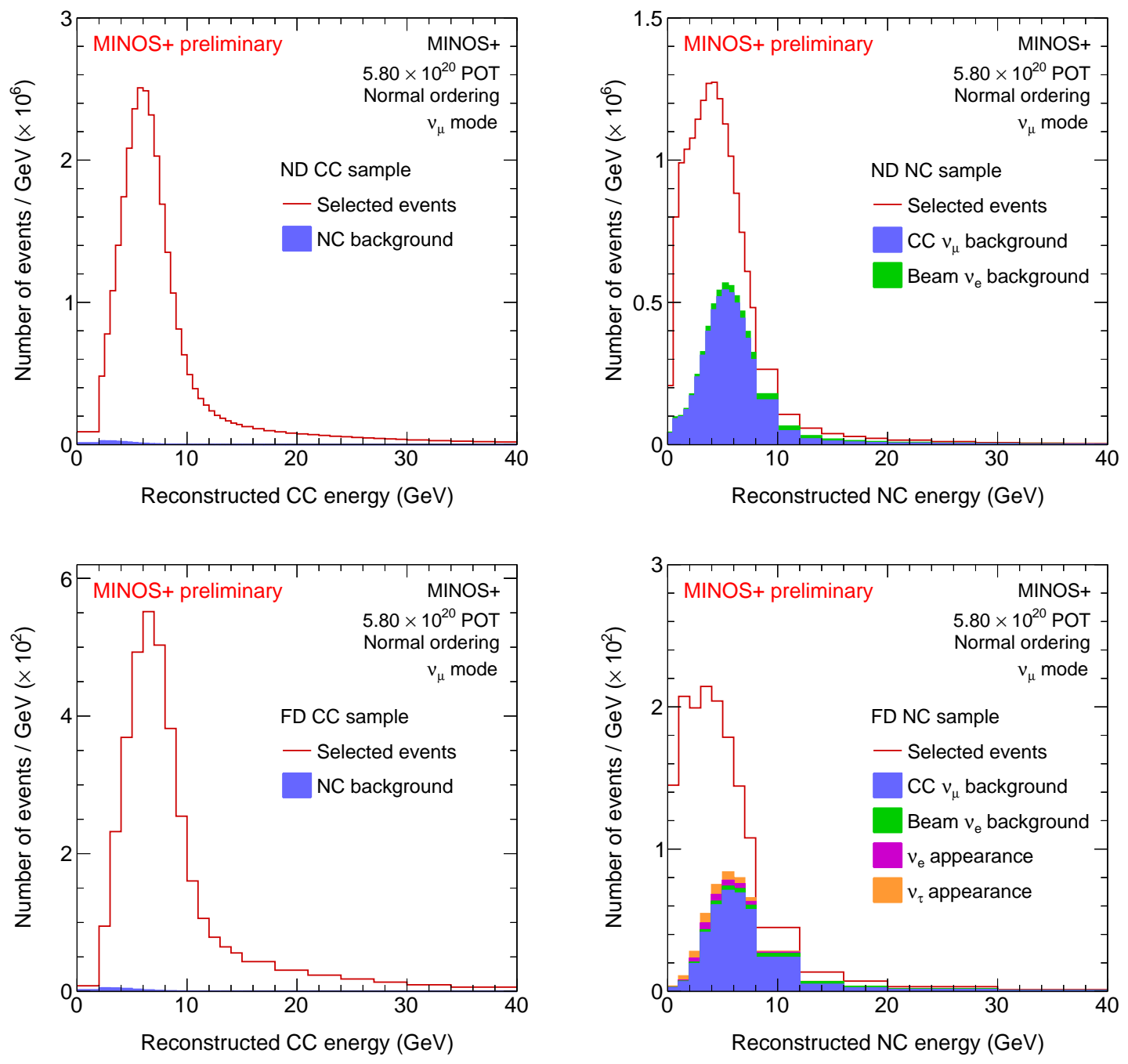

Figure 4.11: Simulated ND (top row) and FD (bottom row) event energy spectra for the CC (left column) and NC (right column) selected samples for MINOS+ only. The spectra are obtained assuming three-flavor oscillations. The main backgrounds are $\mathrm{NC}$ and $\mathrm{CC} \nu_{\mu}$ events for the $\mathrm{CC}$ and $\mathrm{NC}$ samples, respectively. 

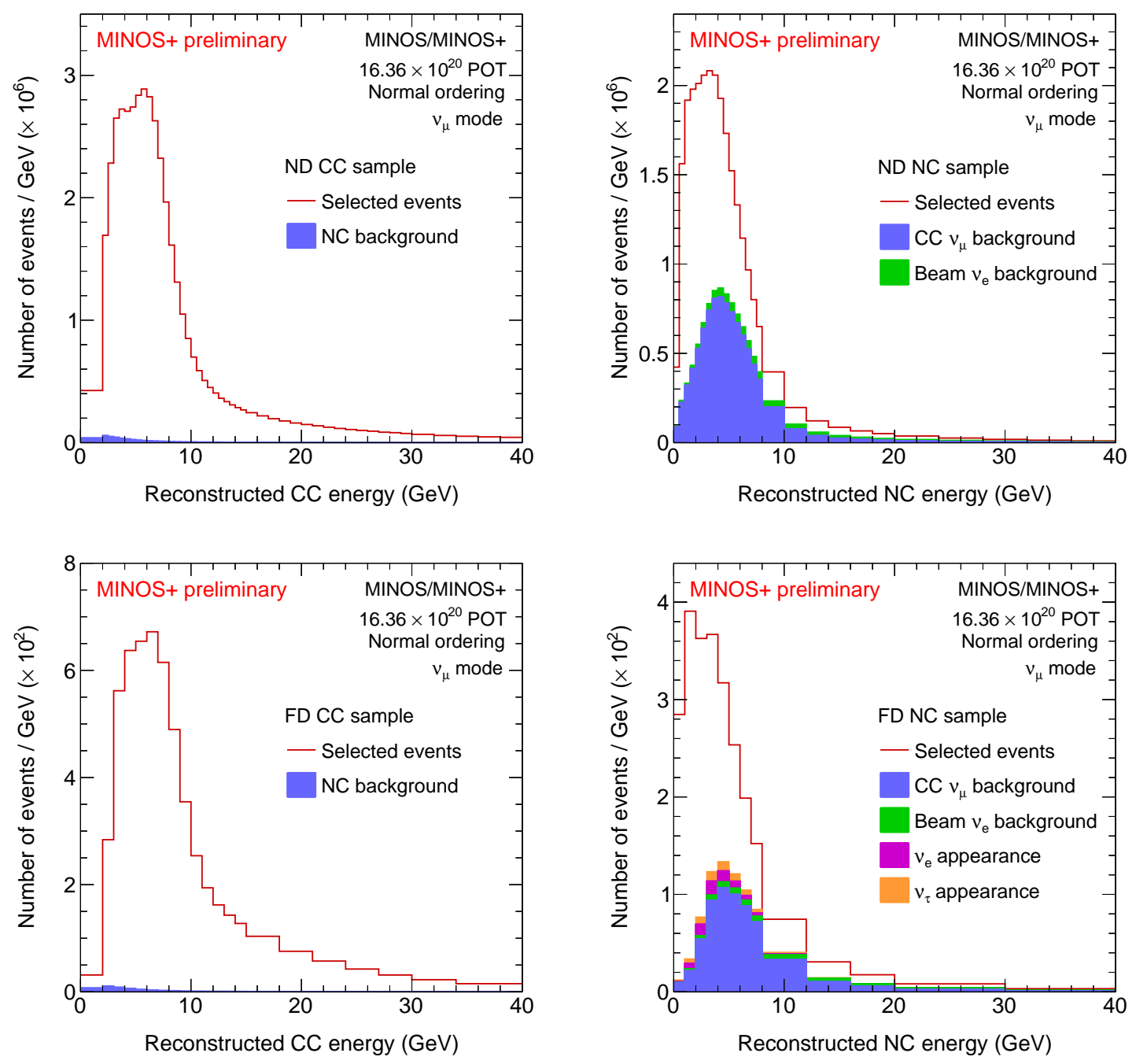

Figure 4.12: Simulated ND (top row) and FD (bottom row) event energy spectra for the CC (left column) and NC (right column) selected samples for MINOS and MINOS+. The spectra are obtained assuming three-flavor oscillations. The main backgrounds are $\mathrm{NC}$ and $\mathrm{CC} \nu_{\mu}$ events for the $\mathrm{CC}$ and $\mathrm{NC}$ samples, respectively. 


\section{Chapter 5}

\section{A Far-Over-Near Analysis}

This chapter presents the Far-over-Near analysis of the LED model based on the full MINOS accelerator beam neutrino dataset with an exposure of $10.56 \times 10^{20} \mathrm{POT}$, for the NuMI beam operated in the low-energy $v_{\mu}$ mode and with a neutrino peak energy of $3 \mathrm{GeV}$. The work in this chapter is a continuation of the work presented in Ref. [45], where a first limit based on the same dataset and obtained using a Far-over-Near analysis strategy was reported. An overview of the analysis method is given in the first section, followed by a discussion of the systematic uncertainties in the second section. Section 5.3 and Section 5.4 show the sensitivities and Feldman-Cousins data limit, respectively. The results of this analysis are published as Rapid Communications in Phys. Rev. D94 (2016) [86]. ${ }^{1}$ The final section takes a first look at MINOS+ data in the context of the Far-over-Near analysis, adding an exposure of $5.80 \times 10^{20} \mathrm{POT}$, for the NuMI beam operated in the medium-energy $v_{\mu}$ mode and with a neutrino peak energy of $7 \mathrm{GeV}$.

\footnotetext{
${ }^{1}$ P. Adamson et al., "Constraints on Large Extra Dimensions from the MINOS Experiment," Phys. Rev., vol. D94, no. 11, p. 111101, 2016. All authors contributed equally.
} 


\subsection{Analysis Method}

As is discussed in Ref. [45], the so-called beam matrix method employed in the MINOS standard oscillations analyses is not suitable in the analysis of a model where neutrino oscillations along the ND baseline are possible. The beam matrix method employs a beam matrix $M$ that converts the ND neutrino flux into an unoscillated FD neutrino flux using the decay kinematics of the hadron parents of the neutrinos, as explained in Ref. [124]. The ND flux is obtained from correcting the ND reconstructed neutrino energy spectrum for simulated ND acceptance and dividing by the calculated cross section. The matrix element $M_{i j}$ gives the relative probability that the hadron parents of neutrinos with energy $E_{i}$ in the ND lead to neutrinos with energy $E_{j}$ in the FD. An inverse correction converts the FD flux to the FD reconstructed neutrino energy spectrum. The neutrino flux simulation is tuned using ND data to optimize the agreement between $\mathrm{MC}$ simulation and data in the ND, as discussed in Section 3.6. This process assumes that there are no neutrino oscillations along the ND baseline, which is valid in the standard oscillation analysis, as is shown Fig. 1.2, but not valid in the LED analysis, as is shown in Fig. 2.9.

The Far-over-Near analysis employs the simulated FLUGG neutrino without any corrections to obtain the unoscillated neutrino energy spectra at the ND and FD. The LED neutrino oscillation probabilities in Formulas (2.4) and (2.5) are then used to calculate the ND and FD reconstructed energy spectra at any point in the LED parameter space. The ratio of the FD and 
ND spectra is used to compare the LED model predictions with the data, both in the $\mathrm{CC}$ and $\mathrm{NC}$ samples.

The use of a Far-over-Near fit provides sensitivity to oscillations along both the ND and FD baselines and significantly reduces many systematic uncertainties affecting both detectors.

\subsubsection{Test Statistic}

A $\chi^{2}$ test statistic is used to compare the predicted and observed Farover-Near ratio in the CC and NC samples. The total $\chi^{2}$ is the sum of those of the CC and NC samples, which are fitted simultaneously

$$
\chi^{2}=\chi_{\mathrm{CC}}^{2}+\chi_{\mathrm{NC}}^{2}
$$

with

$$
\chi_{\mathrm{CC} / \mathrm{NC}}^{2}=\sum_{i, j=1}^{N}\left(o_{i}-p_{i}\right)\left[V^{-1}\right]_{i j}\left(o_{j}-p_{j}\right)+\left(\frac{N_{\mathrm{data}}-N_{\mathrm{MC}}}{\sigma_{N}}\right)^{2}
$$

where $o_{i}$ and $p_{i}$ are the observed and predicted Far-over-Near ratios in energy bin $i$, respectively, and $V$ is the sum of statistical and systematic covariance matrices including bin-to-bin correlations. When comparing a prediction to the data using Formula (5.2), the statistical and systematic uncertainties are those of the prediction. The second term in Formula (5.2) is an ND neutrino flux penalty term, where $N_{\text {data }}\left(N_{\mathrm{MC}}\right)$ is the total number of ND data (MC) events and $\sigma_{N}=50 \% N_{\mathrm{MC}}$ is chosen as a conservative difference between hadron production measurements and MC calculations, as discussed in 
Ref. [45]. Because information on the absolute neutrino event rate is lost when using a Far-over-Near ratio, the aim of the ND neutrino flux penalty term is to constrain the absolute neutrino event rate at any point in the LED parameter space to the measured neutrino event rate in the ND.

Figure 5.1 shows the Far-over-Near ratio for the CC and NC samples for both data, the three-flavor prediction, and the lowest $\chi^{2}$ LED prediction. The energy window for the fit is set between 0 and $40 \mathrm{GeV}$, with the $\mathrm{CC}$ and NC binning schemes chosen such that the minimum number of FD events in a bin provides a good Gaussian approximation.

The atmospheric parameters $\theta_{23}$ and $\Delta m_{32}^{2}$ are free to vary in the fit, thereby minimizing the $\chi^{2}$ value at a fixed point in the $\left(R, m_{0}\right)$ plane. Two initial $\theta_{23}$ hypotheses, one in each octant, are used in the fit. Since the mass ordering is shown to have only small effects on the MINOS sensitivity (Fig. 2.7), only normal ordering is considered in this analysis. The parameters $\theta_{13}, \delta_{\mathrm{CP}}$, $\Delta m_{21}^{2}$, and $\theta_{12}$ have no impact in this analysis (Fig. 2.10 and 2.11) and are fixed to the values shown in Fig. 2.3. As mentioned in Chapter 4, CPT symmetry is assumed, implying identical neutrino and antineutrino oscillation parameters $[125,126]$. The Minuit package [127] is employed to minimize the $\chi^{2}$ value obtained from Formula (5.1).

\subsubsection{Ghost Fitter}

It is clear from Formula (2.3) that the LED oscillation probability is calculated by solving for the eigenvalues of the $n \times n$ Hamiltonian matrix, 


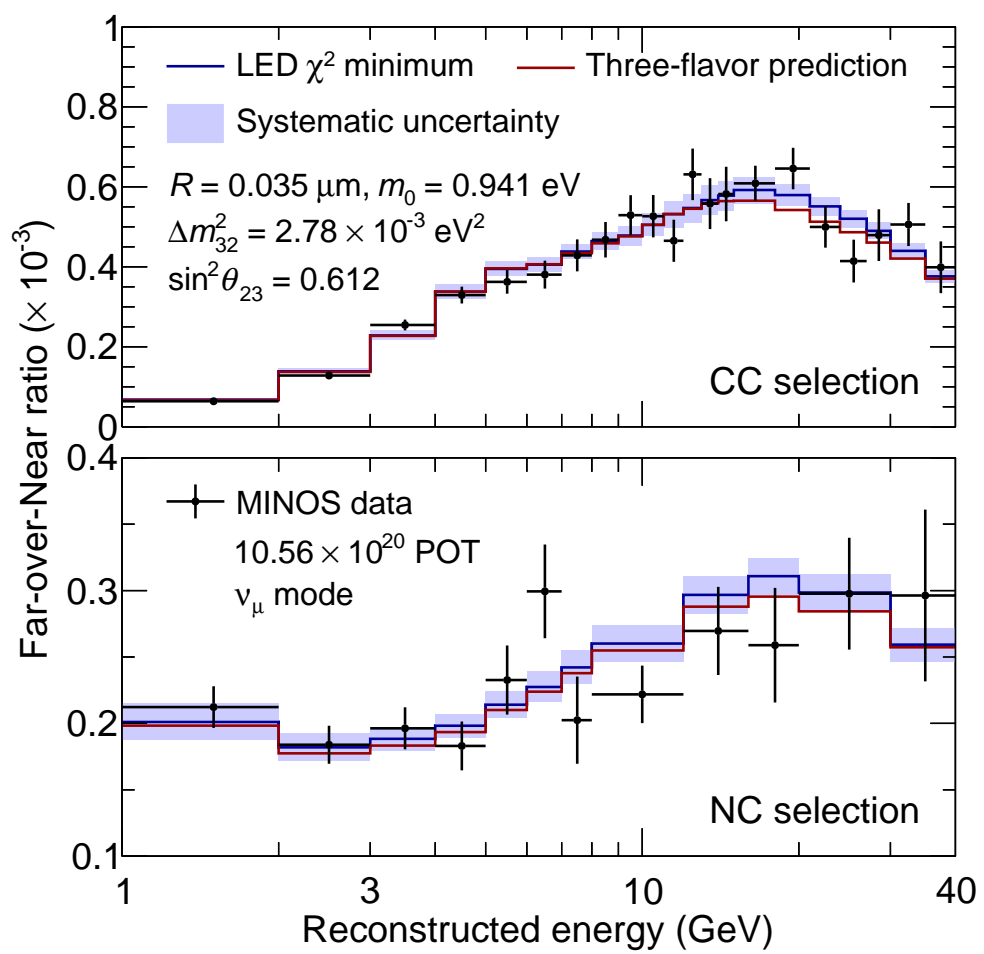

Figure 5.1: The Far-over-Near ratio of the MINOS CC (top) and NC (bottom) data (black points) as a function of the reconstructed neutrino energy. The error bars represent statistical uncertainties. The three-flavor prediction is shown in red. The LED lowest $\chi^{2}$ prediction is shown with its systematic uncertainty (blue line and band), constructed, for illustration purposes, from the square roots of the diagonal elements of the systematic covariance matrix. 
where $n$ is the number of KK modes in all three towers. Although there are an infinite number of KK modes, Figure 2.7 shows that using only the lowest 5 KK modes provides a good approximation, which leads to an $18 \times 18$ matrix. This diagonalization process is performed using the GNU Scientific Library and is very time consuming. To reduce computation time, a Ghost Fitter method was adopted in Ref. [45]. A library of LED predictions is calculated once at the start of the analysis and is called upon by the fitter.

The four-dimensional parameter space is divided into $51 \times 51 \times 26 \times 51$ bins and has ranges $\left[10^{-8}, 10^{-6}\right] \mathrm{m},\left[10^{-3}, 2.5\right] \mathrm{eV},[0, \pi / 2]$, and $\left[10^{-6}, 5 \times 10^{-3}\right] \mathrm{eV}^{2}$ for $R, m_{0}, \theta_{23}$, and $\Delta m_{32}^{2}$, respectively. The Far-over-Near ratio is calculated at each bin center and multilinear interpolation is used to obtain the Far-overNear ratio at other points in the parameter space. The effect of multilinear interpolation on the Far-over-Near ratio depends on $R$ and $m_{0}$ and never exceeds a few percent. This is shown in the context of the two-detector analysis in Fig. 6.3.

Table 5.1 summarizes the values and constraints on the neutrino oscillation parameters used in the LED fitting procedure to minimize Formula (5.1).

A few changes were made to the MC simulation after the publication of Ref. [45] and required the generation of a new prediction library. The corrections to the MC simulation are briefly discussed below. 


\begin{tabular}{|c|c|c|}
\hline Parameter & Free/fixed & Constraint \\
\hline \hline$R$ & Fixed & {$\left[10^{-8}, 10^{-6}\right] \mathrm{m}$} \\
$m_{0}$ & Fixed & {$\left[10^{-3}, 2.5\right] \mathrm{eV}$} \\
$\Delta m_{32}^{2}$ & Free & {$\left[10^{-6}, 5 \times 10^{-3}\right] \mathrm{eV}^{2}$} \\
$\Delta m_{21}^{2}$ & Fixed & $7.54 \times 10^{-5} \mathrm{eV}^{2}$ \\
$\theta_{23}$ & Free & {$[0, \pi / 2]$} \\
$\theta_{13}$ & Fixed & 0.149 \\
$\theta_{12}$ & Fixed & 0.588 \\
$\delta_{\mathrm{CP}}$ & Fixed & 0 \\
\hline
\end{tabular}

Table 5.1: The values and constraints on the neutrino oscillation parameters used in the LED fitting procedure that minimizes Formula (5.1).

\subsubsection{Corrections to the Monte Carlo Simulation}

Two corrections were applied to the MC simulation compared to the Far-over-Near analysis presented in Ref. [45].

Firstly, it was discovered that the normalization strategy used for the MINOS MC samples was not optimal, with the samples from of the individual MINOS run periods first added together and then normalized to the total data POT. A more precise treatment is to normalize the MC samples individually to the corresponding data run period POT and then add them together. The top panels in Fig. 5.2 show the effect of the new normalization strategy on the Farover-Near ratio in the $\mathrm{CC}$ and $\mathrm{NC}$ samples for a point in the LED parameter space. The effect is approximately independent of the LED parameters and largest below $6 \mathrm{GeV}$, peaking at $3 \%$ in the $\mathrm{CC}$ sample and $4 \%$ in the $\mathrm{NC}$ sample. 
Secondly, it was found that the MINOS MC samples were created using a NuMI beam target $Z$ position that does not represent the actual position of the target at the time of data taking. A target $Z$ position shift of $1.25 \mathrm{~cm}$ is applied to fix this. The bottom panels in Fig. 5.2 show the effect of this shift on the Far-over-Near ratio in the $\mathrm{CC}$ and $\mathrm{NC}$ samples for a point in the LED parameter space. The effect is approximately independent of the LED parameters and peaks at $4 \%$ in the CC sample and remains below $1 \%$ in the NC sample.

\subsubsection{Dynamic Scaling of the Covariance Matrix}

In Ref. [45], the statistical and systematic contributions to the $i j^{\text {th }}$ element of the covariance matrix are given by

$$
\begin{aligned}
V_{i j}^{\text {stats }} & =\left[\frac{p_{i}^{3 v}}{N_{i}^{3 v}}+\frac{\left(p_{i}^{3 v}\right)^{2}}{N_{i}^{3 v}}\right] \delta_{i j} \approx \frac{p_{i}^{3 v}}{N_{i}^{3 v}} \delta_{i j}, \\
V_{i j}^{\text {systs }} & =p_{i}^{3 v} U_{i j}^{\text {systs }} p_{j}^{3 v},
\end{aligned}
$$

with $p_{i}^{3 v}$ and $N_{i}^{3 v}$ the predicted Far-over-Near ratio and number of ND events, respectively, for the standard three-flavor prediction in energy bin $i$. The fractional systematic covariances in the Far-over-Near ratio are denoted by $U_{i j}^{\text {systs }}$ and are discussed in more detail in Section 5.2. Because the threeflavor prediction is fixed, the total covariance matrix is the same for each LED prediction. This is called static scaling.

However, the predictions in the LED parameter space can be significantly different from the three-flavor prediction. A more correct approach to 


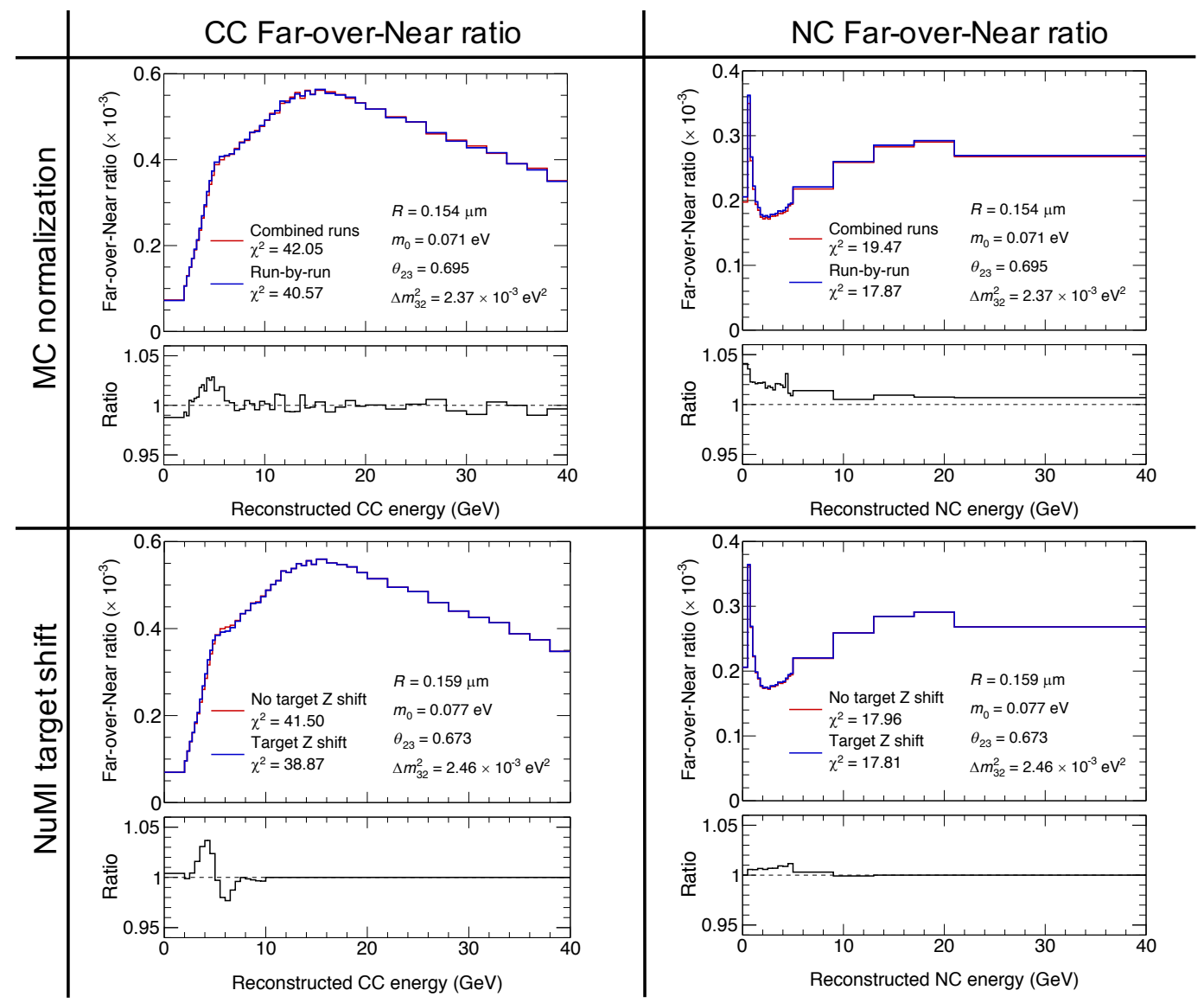

Figure 5.2: Corrections to the MC samples. (Top row) The effect of changing from an MC sample normalization by total data POT (red line) to a sampleby-sample normalization by data run period POT (blue line) on the CC (left) and NC (right) samples. (Bottom row) The effect of a $1.25 \mathrm{~cm}$ NuMI beam target $Z$ position shift on the $\mathrm{CC}$ (left) and $\mathrm{NC}$ (right) samples. 
scale the fractional covariance matrices would be to use the LED prediction rather than the three-flavor prediction. This is called dynamic scaling, given the covariance matrix is different at each point in the LED parameter space and leads to covariance matrix elements

$$
\begin{aligned}
V_{i j}^{\text {stats }} & =\left(\frac{p_{i}}{N_{i}^{\text {unosc }}}+\frac{p_{i}{ }^{2}}{N_{i}^{\text {unosc }}}\right) \delta_{i j} \approx \frac{p_{i}}{N_{i}^{\text {unosc }}} \delta_{i j}, \\
V_{i j}^{\text {systs }} & =p_{i} U_{i j}^{\text {systs }} p_{j} .
\end{aligned}
$$

It should be noted that the statistical variance for dynamic scaling in Formula (5.5) uses the unoscillated ND prediction rather than the LED ND prediction in the denominator. Using the latter would result in decreasing $\chi^{2}$ values in regions of the parameter space where significant oscillations along the ND baseline occur, as is illustrated below.

As discussed in Ref. [128], the fractional error on the Far-over-Near ratio in any energy bin, now for convenience defined as $R=F / N$, is given by

$$
\begin{aligned}
\left(\frac{\sigma_{R}}{R}\right)^{2} & =\left(\frac{\sigma_{F}}{F}\right)^{2}+\left(\frac{\sigma_{N}}{N}\right)^{2} \\
& =\left(\frac{\sqrt{F}}{F}\right)^{2}+\left(\frac{\sqrt{N}}{N}\right)^{2} \\
& =\frac{1}{F}+\frac{1}{N} \\
& \approx \frac{1}{F},
\end{aligned}
$$

where in the last step the $1 / N$ term can be left out because the number of events in the ND is much larger than in the FD. The variance in $R$ can be rewritten in terms of $R$ and $N$ as

$$
\sigma_{R}^{2} \approx \frac{R}{N}
$$


Notice that this is the same result as in Formula (5.3), when using the three-flavor Far-over-Near ratio $R_{3}$ and ND spectrum $N_{3}$. To obtain Formula (5.5) for dynamic scaling at a point in the LED parameter space, consider the following: naively one would expect the prediction at the ND and FD to be

$$
\begin{aligned}
& N_{\mathrm{LED}}=P_{N} N_{\text {unosc }}, \\
& F_{\mathrm{LED}}=P_{F} F_{\text {unosc }},
\end{aligned}
$$

where $P_{N}$ and $P_{F}$ are the LED oscillation probabilities at the ND and FD, respectively, and $N_{\text {unosc }}$ and $F_{\text {unosc }}$ are the unoscillated predictions at the ND and FD, respectively, such that the Far-over-Near ratio is given by

$$
R_{\mathrm{LED}}=\frac{P_{F}}{P_{N}} \frac{F_{\text {unosc }}}{N_{\text {unosc }}} .
$$

It should be noted that $N_{\text {unosc }}=N_{3}$, given the MINOS ND baseline was chosen such that three-flavor oscillations are not significant. However, Formulas (5.9) and (5.10) are not correct. At the ND, it is known exactly how many events are observed and the ND prediction must always be equal to this at any point in the LED parameter space. When oscillations occur along the ND baseline, this implies that muon neutrinos have disappeared due to oscillations but the underlying neutrino flux and cross sections have increased to compensate such that $N_{\mathrm{LED}}=N_{\text {unosc }}$ (which is also encapsulated in the ND neutrino flux penalty term in Formula (5.2)). As such, the FD prediction requires the same compensation and the correct predictions are

$$
N_{\mathrm{LED}}=N_{\mathrm{unosc}}
$$




$$
F_{\mathrm{LED}}=\frac{P_{F}}{P_{N}} F_{\text {unosc }} .
$$

The Far-over-Near ratio, $R_{\mathrm{LED}}$, is still given by Formula (5.11). However, using Formula (5.12) in Formula (5.8) gives the statistical variance for the LED prediction

$$
\sigma_{R_{\mathrm{LED}}}^{2} \approx \frac{R_{\mathrm{LED}}}{N_{\text {unosc }}},
$$

which is the same result as in Formula (5.5). It should be noted that the detector resolution, which smears the probability as a function of energy, has been ignored in this derivation of the statistical variance. However, this is only a small second order effect on the sizes of the variances.

A visual comparison of static and dynamic scaling is shown in Fig. 5.3, using the full Far-over-Near analysis software for a toy model case with flat ND and FD simulated data spectra and predictions and assuming statistical uncertainties only. The number of events per energy bin for the flat spectra are listed in Table 5.2. The top left panel of Fig. 5.3 shows static scaling applied to the three-flavor prediction (red line). The magnitude of the uncertainty band in the three-flavor prediction is given by the square root of the statistical variance in the three-flavor prediction, calculated using the three-flavor Farover-Near ratio and ND prediction. The $\chi^{2}$ calculation for these simple cases reduces to

$$
\chi^{2}=n \frac{(o-p)^{2}}{\sigma^{2}},
$$

where $n$ is the number of bins $(n=48), o$ and $p$ the Far-over-Near ratio in one bin for the simulated data and prediction, respectively, and $\sigma$ the statistical 


\begin{tabular}{|c|c|c|c|}
\hline Type & FD events & ND events & FD/ND \\
\hline \hline Simulated data & 100 & $4 \times 10^{5}$ & $2.5 \times 10^{-4}$ \\
3-flavor prediction & 44 & $2 \times 10^{5}$ & $2.2 \times 10^{-4}$ \\
LED prediction & 20 & $1 \times 10^{5}$ & $2.0 \times 10^{-4}$ \\
\hline
\end{tabular}

Table 5.2: The number of events and Far-over-Near ratio in each energy bin for the toy model spectra used in Fig. 5.3 to illustrate static versus dynamic scaling for the statistical variance.

uncertainty on the Far-over-Near ratio prediction. The top right panel shows static scaling applied to the LED prediction (blue line). In this case, the uncertainty in the LED prediction (blue band) is the same as the uncertainty in the three-flavor prediction in the top left panel (red band), but centered around the LED prediction. As expected, the $\chi^{2}$ is larger than in the top left panel because the prediction is further from the simulated data. The bottom panels show dynamic scaling applied to the LED prediction. In the left bottom panel, the LED ND prediction is employed to calculate the statistical variance used in Formula (5.15), while the three-flavor ND prediction is employed in the right bottom panel. The $\chi^{2}$ value in the left bottom panel is much lower than the $\chi^{2}$ value in the right bottom panel. It can be readily inferred that the statistical uncertainties blow up in regions of the parameter space where the ND oscillation probability increases and cause problems in the fitting procedure as artificial minima will arise in those regions. 

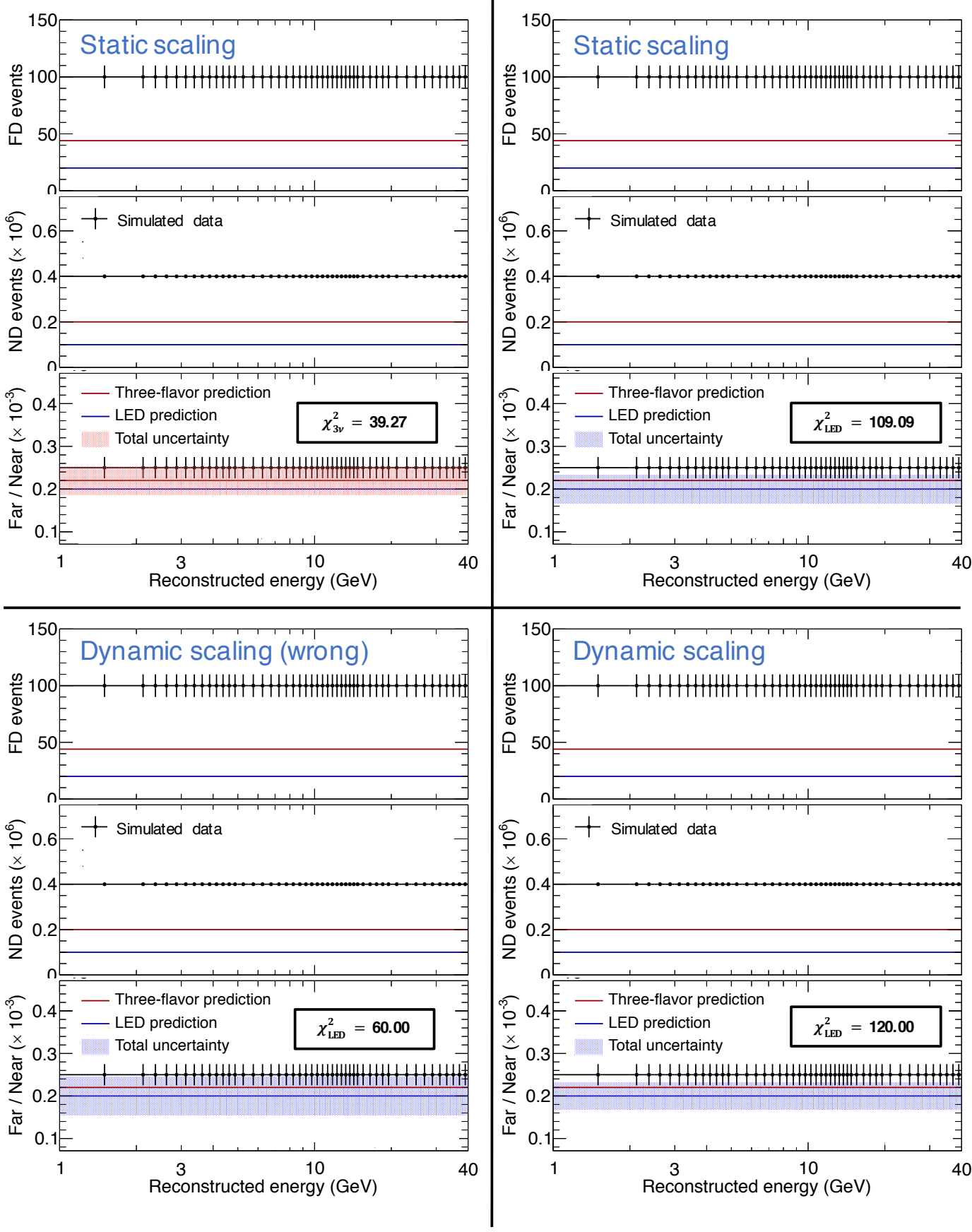

Figure 5.3: A toy model example to illustrate the importance of using the correct statistical uncertainty in the dynamic scaling procedure, comparing static scaling for the three-flavor prediction (top left) to static scaling (top right), dynamic scaling using the LED ND prediction (bottom left), and dynamic scaling using the three-flavor ND prediction (bottom right) for the LED prediction. 


\subsection{Systematic Uncertainties}

The systematic uncertainties that could affect the predicted energy spectra used in the Far-over-Near analysis are converted into covariance matrices for the CC and NC Far-over-Near ratio samples. The total covariance matrix $V$ used in Formula (5.2) for a given LED model prediction is given by the sum of the statistical and systematic covariance matrices

$$
V=V_{\mathrm{stat}}+\sum_{k=1}^{8} V_{\mathrm{syst}, k},
$$

where the systematic uncertainties for the Far-over-Near analysis are grouped

into 8 categories. The $i j^{\text {th }}$ elements of $V_{\text {stat }}$ and $V_{\text {syst }, k}$ are given by Formula (5.5) and (5.6), respectively.

The Far-over-Near ratio method has the advantage of being robust to systematic uncertainties due to an explicit cancellation in the ratio of systematic uncertainties that are correlated in both detectors, including the crosssection, beam, and reconstruction uncertainties.

Following the work in Ref. [46, 129], this section discusses the construction of the fractional systematic covariance matrices $U_{i j}$ from Formula (5.5) for the MINOS Far-over-Near analysis.

\subsubsection{Constructing Uncertainty Bands}

Two approaches are followed to construct the systematic uncertainty bands. All but the acceptance systematic uncertainty are evaluated using both 
the FD and ND MC samples through the double ratio

$$
\frac{(\mathrm{FD} / \mathrm{ND})_{\text {shifted }}}{(\mathrm{FD} / \mathrm{ND})_{\text {nominal }}}-1
$$

where $(\mathrm{FD} / \mathrm{ND})_{\text {nominal }}$ is the ratio of the FD and ND three-flavor MC samples used in the Far-over-Near analysis and $(\mathrm{FD} / \mathrm{ND})_{\text {shifted }}$ is the ratio of the FD and ND MC samples obtained for adjusted conditions related to the systematic uncertainty.

The acceptance systematic uncertainty, which reflects the mismodeling of the MC simulation due to event selections, is evaluated using the ND MC and data samples through the double ratio

$$
\frac{(\mathrm{ND} \text { data/ND } \mathrm{MC})_{\text {shifted }}}{(\mathrm{ND} \text { data/ND } \mathrm{MC})_{\text {nominal }}}-1
$$

where the event selection requirements are varied to obtain (ND data/ND MC) shifted. $_{\text {. }}$

For the cases that do not naturally produce a lower and upper uncertainty band, the negative of the uncertainty band is employed to construct symmetrical lower and upper uncertainty bands.

\subsubsection{Constructing Covariance Matrices}

To convert the uncertainty bands obtained from Formula (5.17) or (5.18) into a covariance matrix, a set of $N$ linearly interpolated or extrapolated uncertainties $\epsilon_{i, k}(k=1, \ldots, N)$ in reconstructed energy bin $i$ are used such that

$$
V_{i j}=\frac{1}{N} \sum_{k=1}^{N} \epsilon_{i, k} \epsilon_{j, k}
$$


For a set of random numbers $g_{k}(k=1, \ldots, N)$ drawn from a Gaussian distribution with $\mu=0$ and $\sigma=1$, linear interpolation between the lower and upper uncertainty bands, $e_{\text {lower }, i}$ and $e_{\text {upper }, i}$, is employed when $-1<g_{k}<1$

$$
\epsilon_{i, k}=\frac{1}{2} g_{k}\left(g_{k}-1\right) e_{\text {lower }, i}+\frac{1}{2} g_{k}\left(g_{k}+1\right) e_{\text {upper }, i}
$$

while linear extrapolation is used when $\left|g_{k}\right| \geq 1$.

\subsubsection{Total Systematic Uncertainty}

The same systematic uncertainties as in Ref. [45] are used for the MINOS Far-over-Near analysis. Figure 5.4 shows the total CC and NC systematic uncertainty bands and their corresponding covariance matrices. The individual systematic uncertainty sources are discussed below.

\subsubsection{Acceptance Uncertainty}

The acceptance uncertainty provides an estimate of the agreement between data and $\mathrm{MC}$ simulation by looking at different parts of the detectors. The uncertainty in the acceptance and event selection efficiency for the ND $\mathrm{CC}$ and $\mathrm{NC}$ samples is evaluated by varying event selection requirements in data and MC simulation. Any shift in the agreement between data and MC is taken as a systematic uncertainty through Formula (5.18). Variations in the event selection requirements include:

- selecting only events in the left or right half of the ND fiducial region for the $\mathrm{CC}$ and $\mathrm{NC}$ samples, 

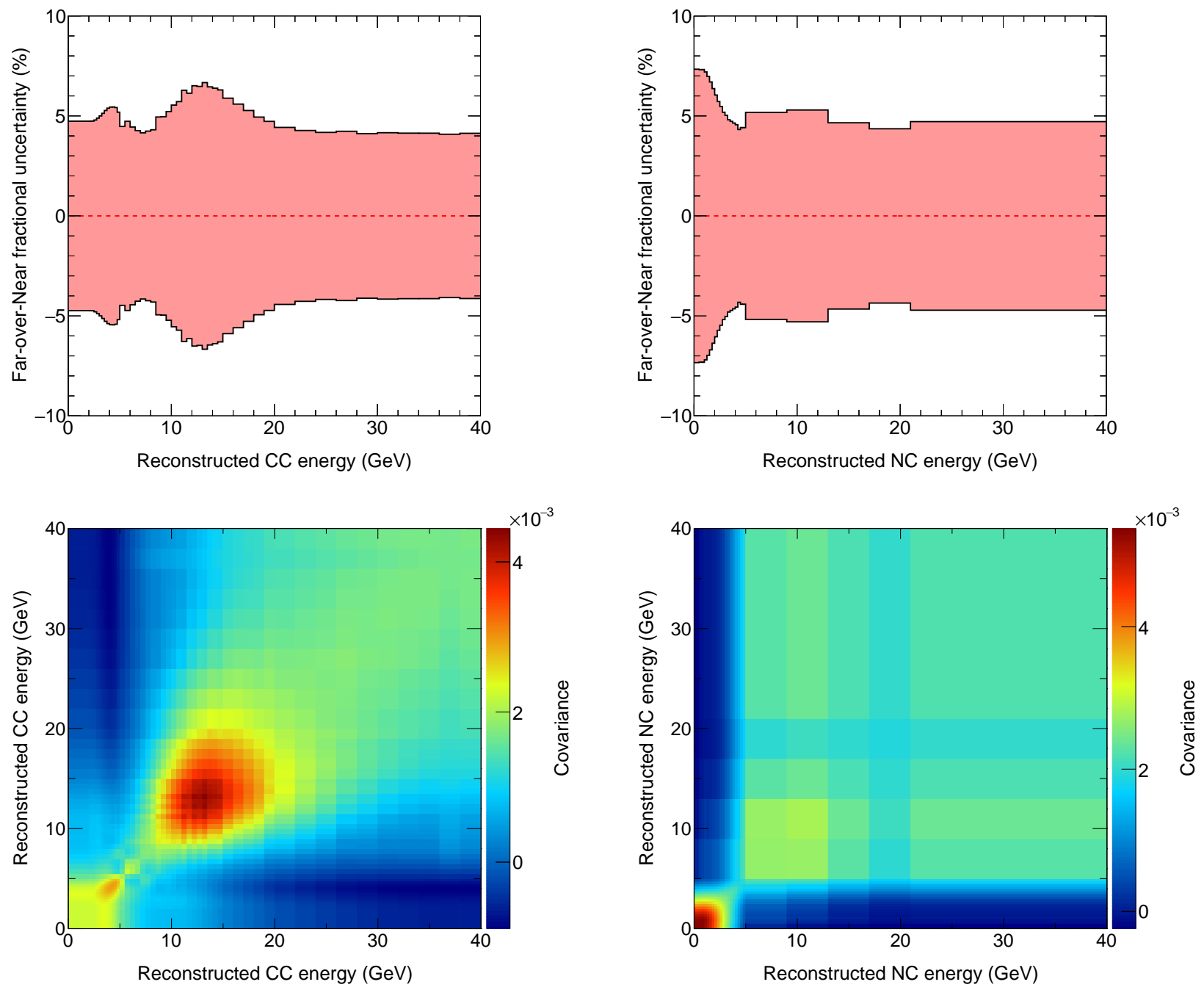

Figure 5.4: The total systematic uncertainty for the MINOS samples. The fractional uncertainty in the CC (top left) and NC (top right) Far-over-Near ratios and the corresponding covariance matrices for the $\mathrm{CC}$ (bottom left) and NC (bottom right) samples. 
- changing the endpoint along the NuMI beamline of the ND fiducial region from $4.7368 \mathrm{~m}(4.077 \mathrm{~m})$ to $2.5 \mathrm{~m}$ for the $\mathrm{NC}(\mathrm{CC})$ sample,

- varying the radius of the ND fiducial region from $80 \mathrm{~cm}$ to $60 \mathrm{~cm}$ for the CC sample,

- including CC events with tracks that cross the ND coil hole,

- removing events with a track that ends within 10 scintillator planes of the start of the ND spectrometer part (planes 121-281, where only every fifth steel plane is accompanied by a scintillator plane) for the $\mathrm{CC}$ sample,

- including events with a track that exits through the side of the ND calorimeter part (first 120 planes, where each steel plane is accompanied by a scintillator plane) for the CC sample,

- removing events with a track that ends within 10 scintillator planes of the end of the ND for the CC sample.

Figure 5.5 shows the total fractional acceptance uncertainty in the Far-overNear CC and NC ratios obtained from adding in quadrature the individual fractional uncertainties from the variations listed above. The acceptance systematic is energy dependent and includes correlations between different energy bins, as can be seen from the covariance matrices shown in the bottom pannels of Fig. 5.5. The magnitude of the acceptance uncertainty is larger for the CC sample than for the NC sample due to events with long tracks in the ND for the CC sample. 

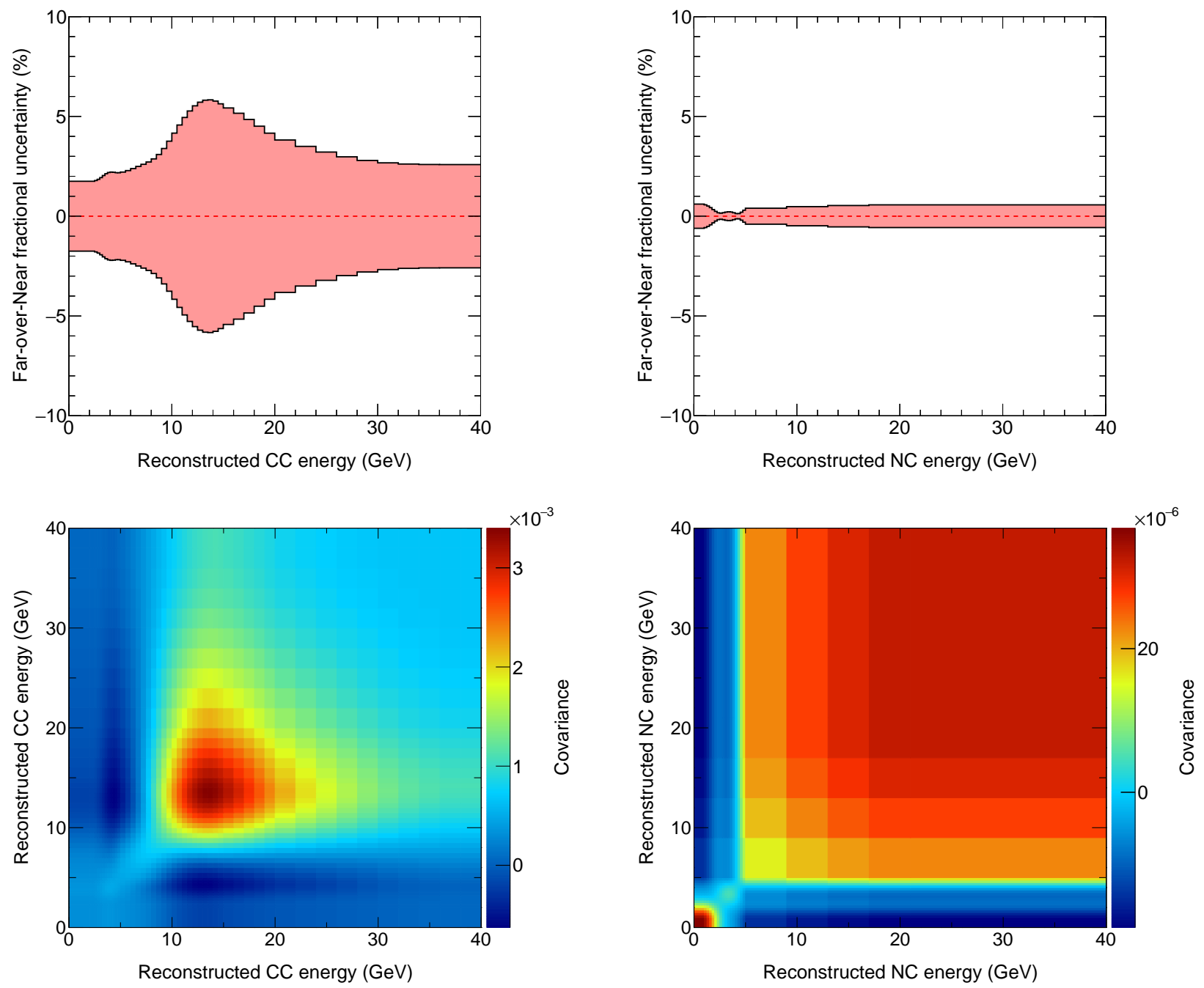

Figure 5.5: The acceptance systematic uncertainty for the MINOS samples. The fractional uncertainty in the CC (top left) and NC (top right) Far-overNear ratios and the corresponding covariance matrices for the CC (bottom left) and NC (bottom right) samples. 


\subsubsection{Normalization Uncertainty}

The normalization uncertainty quantifies the biases and uncertainties associated with measuring the total data exposure in the ND and FD. It is a consequence of the detector differences between the ND and FD, including steel and scintillator dimensions, detector live time, and event reconstruction efficiencies.

An NC reconstruction efficiency is evaluated in both detectors by selecting $\mathrm{CC}$ events that successfully passed the $\mathrm{CC}$ event selection requirements and removing the muon track from those events to mimmic NC events. By pass-

ing these muon-removed $\mathrm{CC}$ events through the reconstruction chain again, the reconstruction efficiencies are calculated, resulting in a $2 \%$ normalization uncertainty. This is added in quadrature with the uncertainties in steel and scintillator thicknesses, and the uncertainties in the determination of the FD live time, to obtain a $2.2 \%$ energy independent $\mathrm{NC}$ normalization uncertainty.

A $1.6 \%$ CC normalization uncertainty across all energies is obtained from a careful identification of $\mathrm{CC}$ events in data and $\mathrm{MC}$ samples for both detectors that failed the event reconstruction process or were not fully contained in the fiducial volume.

The total fractional normalization uncertainty in the Far-over-Near $\mathrm{CC}$ and $\mathrm{NC}$ ratios and their corresponding covariance matrices are shown in Fig. 5.6. 

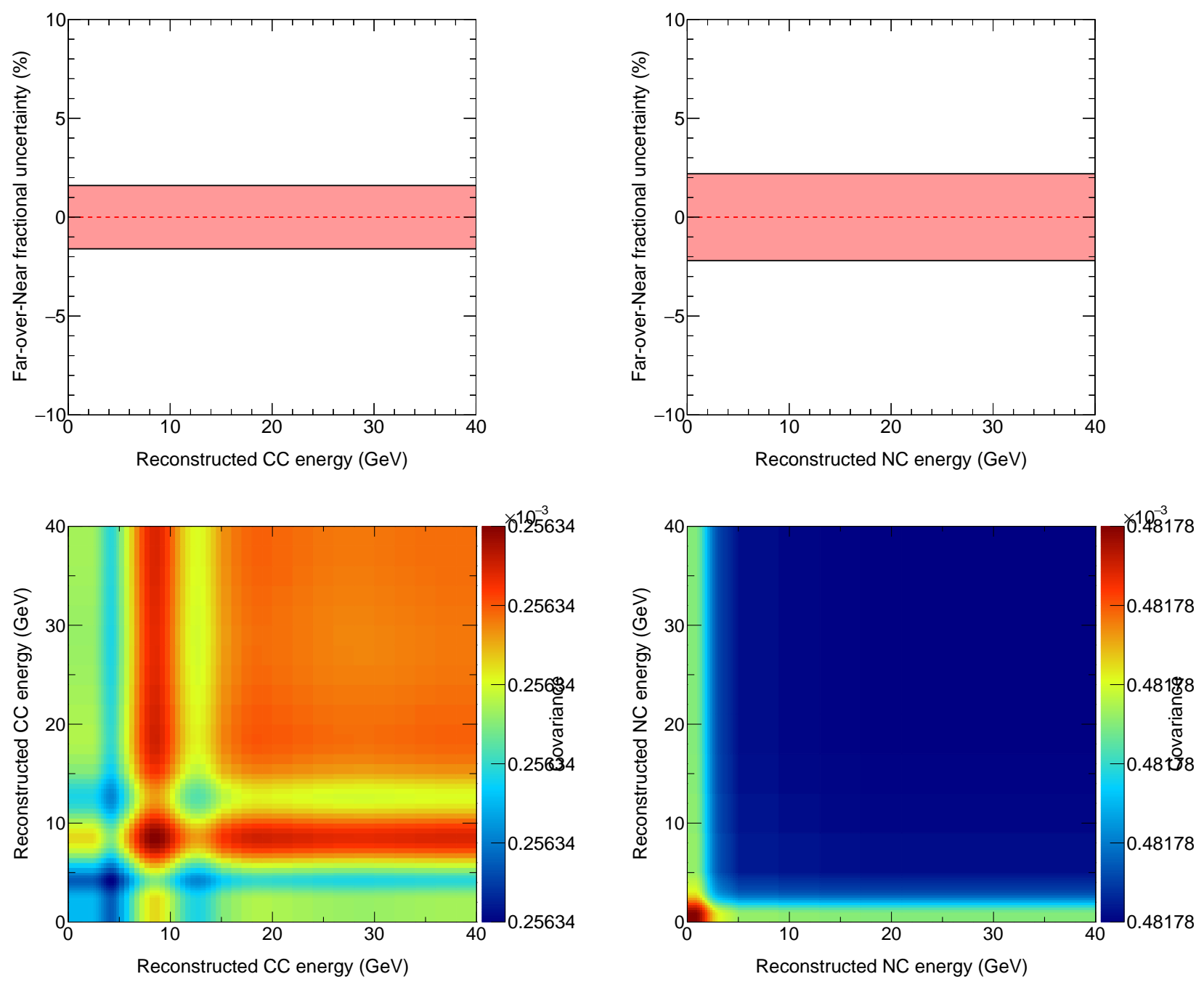

Figure 5.6: The normalization systematic uncertainty for the MINOS samples. The fractional uncertainty in the CC (top left) and NC (top right) Far-overNear ratios and the corresponding covariance matrices for the CC (bottom left) and $\mathrm{NC}$ (bottom right) samples. 


\subsubsection{Neutral Current Selection Uncertainty}

The NC selection uncertainty accounts for the uncertainty in the preselection procedure that reduces the number of poorly reconstructed $\mathrm{NC}$ events in the ND, discussed in Section 4.1. The preselection requirements are varied to let the fraction of poorly reconstructed events in the $\mathrm{MC}$ simulation match with data in the ND, where for data the nominal selection requirements are always used. A more detailed discussion is found in Ref. [121].

In the FD, noise, light pulses from the LI system, and cosmic muons that enter the fiducial volume at steep angles can be reconstructed as $\mathrm{NC}$ events. An uncertainty is assigned to the removal procedure of such events.

Figure 5.7 shows the NC selection uncertainty in the Far-over-Near ratio and its corresponding covariance matrix. The NC selection procedure tends to remove poorly reconstructed events in the low-energy bins, such that the largest uncertainties associated with the selection requirements are at lower energies and decrease with increasing energy.

\subsubsection{Energy Scale Uncertainties}

The energy scale uncertainties in the CC and NC Far-over-Near ratios are shown in Fig. 5.8. The energy scale uncertainties consist of the muon track and shower energy uncertainties. The muon track energy uncertainty is $2 \%(3 \%)$ when the muon momentum is determined from its track range (curvature) [122]. The hadronic shower energy has two sources of uncertainty, 

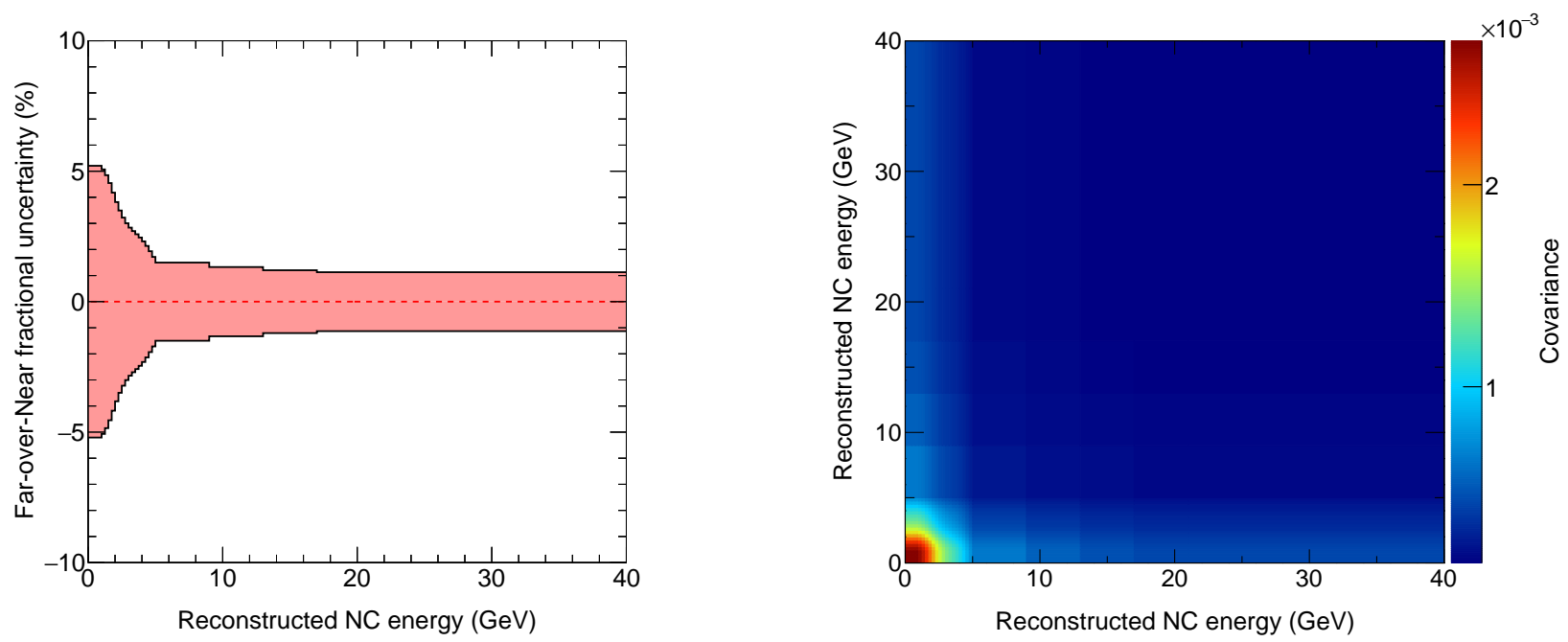

Figure 5.7: The NC selection systematic uncertainty for the MINOS sample. The fractional uncertainty in the NC Far-over-Near ratio (left) and the corresponding covariance matrix (right).

the relative and absolute energy scale uncertainties, for which the individual contributions are listed in Table 5.3 and 5.4, respectively, and discussed below.

\section{Absolute Energy Scale Uncertainty}

The absolute energy scale uncertainties are determined from the analysis of CalDet data and cosmic muon data in the ND and FD. The discrepancy between the response to individual hadrons measured in CalDet and in MC simulation is 5\% [78], consistent with model uncertainties [130]. The MEU constant in CalDet was measured at CERN using beam muons and has an uncertainty of $1.4 \%$ [103]. Further studies of the stopping muon track end point indicated an inherent uncertainty in the beam energy of $2 \%$, which con- 

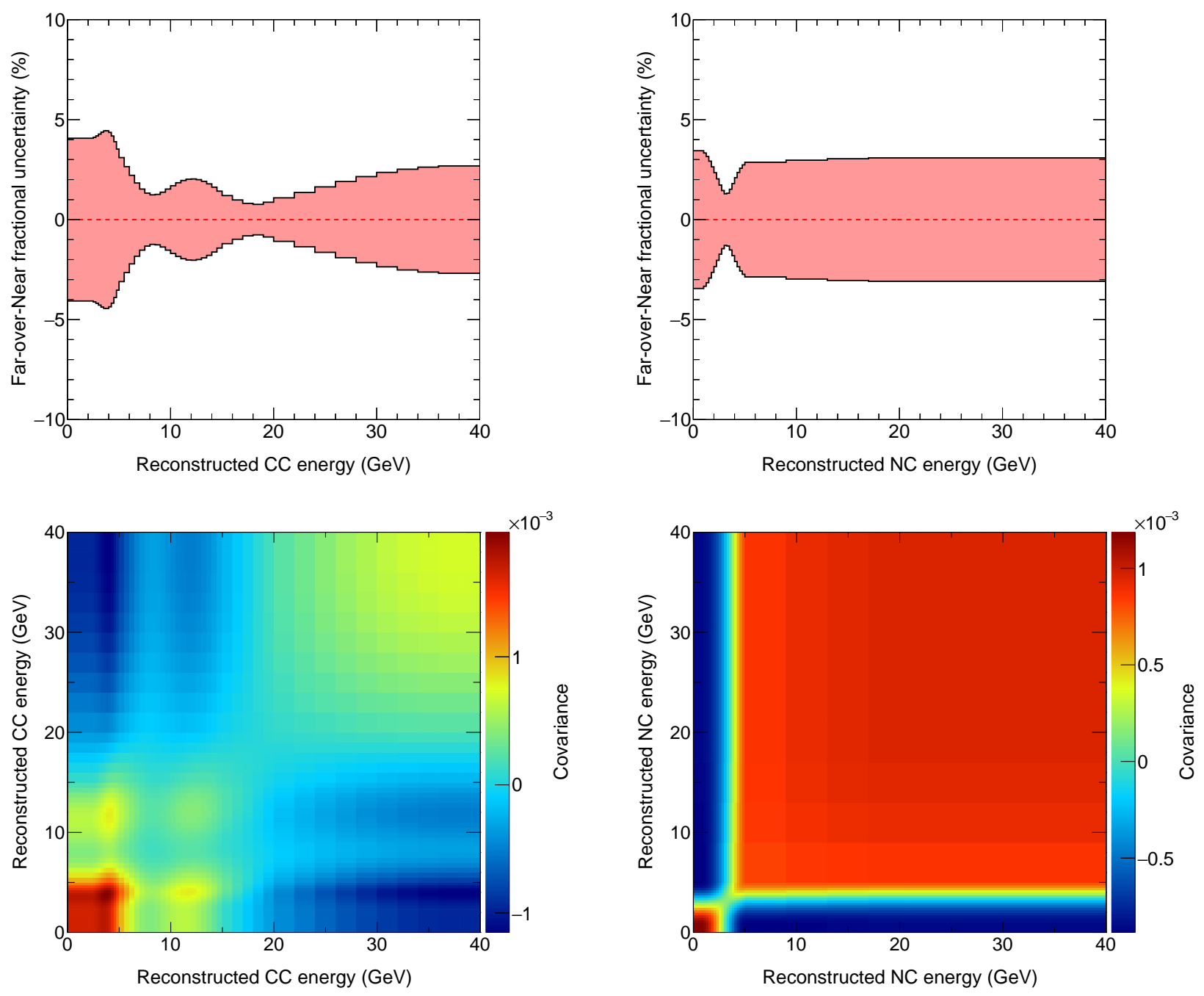

Figure 5.8: The energy scale systematic uncertainties for the MINOS samples. The fractional uncertainty in the CC (top left) and NC (top right) Far-overNear ratios and the corresponding covariance matrices for the CC (bottom left) and $\mathrm{NC}$ (bottom right) samples. 


\begin{tabular}{|l|c|}
\hline Source & $\begin{array}{c}\text { Energy scale } \\
\text { uncertainty }\end{array}$ \\
\hline \hline Tuning hadron MC to CalDet data & $5 \%$ \\
CalDet beam energy uncertainty & $2 \%$ \\
CalDet stopping muon calibration uncertainty & $1.4 \%$ \\
ND/FD light level difference & $1 \%$ \\
ND MEU value from beam induced muons & $0.8 \%$ \\
\hline Total & $5.7 \%$ \\
\hline
\end{tabular}

Table 5.3: Systematic uncertainties in the absolute energy scale derived from CalDet data and cosmic muon data in the ND and FD.

\begin{tabular}{|l|c|c|}
\hline Calibration & ND & FD \\
\hline \hline Drift & $0.91 \%$ & $0.38 \%$ \\
Linearity & $0.15 \%$ & $0.15 \%$ \\
Strip-to-strip & $0.74 \%$ & $0.35 \%$ \\
Noise & $0 \%$ & $0.1 \%$ \\
Random & $0.2 \%$ & $0.2 \%$ \\
\hline \multicolumn{3}{|c|}{ Stopping muon uncertainties } \\
\hline \hline Shape & $0.35 \%$ & $0.21 \%$ \\
Spatial & $0.68 \%$ & $0.76 \%$ \\
Beam vs cosmic & $1.03 \%$ & - \\
Tuning to MC & $0.01 \%$ & $0.21 \%$ \\
Statistical & $0.01 \%$ & $0.02 \%$ \\
\hline Total & $1.8 \%$ & $1.0 \%$ \\
\hline
\end{tabular}

Table 5.4: Systematic uncertainties in the relative energy scale determination at the ND and FD. 
tributes a $2 \%$ uncertainty in the hadronic energy scale. The net uncertainty in the absolute energy scale measured at CalDet is $5.6 \%$.

Since the absolute energy scale is set by CalDet, the degree by which it was tuned such that muons observed at CalDet look the same as muons at the ND and FD gives rise to an uncertainty in the absolute energy scale. The light levels differ by $0.8 \%$ in the ND and FD and this value is used as a systematic uncertainty in the absolute energy scale. Furthermore, the MEU constant obtained from stopping muons resulting from NuMI beam neutrino interactions at the ND is $1 \%$ higher for data than for $\mathrm{MC}$ simulation (no such comparison is possible at the FD due to limited neutrino event counts). This difference is taken as an additional systematic uncertainty in the absolute energy scale. These two systematic uncertainties are combined with those from the absolute energy scale measured at CalDet to give an absolute energy scale uncertainty of $5.7 \%$.

\section{Relative Energy Scale Uncertainty}

The degree to which changes to the individual calibration steps, outlined in Section 3.5, affect the MEU values are taken as systematic uncertainties in the relative energy scale. Given that all calibration constants are multiplicative, the systematic uncertainty in the energy scale in each detec-

tor is the quadratic sum of individual calibration uncertainties, with a $1.8 \%$ uncertainty in the ND and a $1.1 \%$ uncertainty in the FD. 
As discussed in Section 3.5.2.1, the drift correction is determined using the median detector response. Alternatively, the mean response can be used. This leads to a different drift correction because the shape of the distribution from which the mean and median are extracted is not constant with time due to the aging of the detectors. The maximum deviation in the ratio of these corrections from unity, after taking into account statistical uncertainties, is taken as the systematic uncertainty.

As discussed in Section 3.5.3, the strip-to-strip calibration constants are normalized to a predetermined median calibration constant, rather than a median response, to mitigate the impact of aging effects. Half of the variation in the ratio of median to mean strip-to-strip constants is taken as the systematic uncertainty. The MEU constants are calculated assuming a particular fiducial volume, while the strip-to-strip calibration calibrates every strip in each detector. Because individual strips aged differently, the fiducial volume aged differently than the complete detector. To estimate a systematic uncertainty due to fiducial volume selection requirements, the median calibration constant derived using only strips in the fiducial volume is compared to that derived using the complete detector and half the difference is assigned as systematic uncertainty. The different systematic uncertainty sources for the strip-to-strip calibration are added in quadrature.

The PMTs typically showed linear behavior up to several hundred PEs [94] and the non-linearity varied by $20 \%$ from channel to channel. In the energy region where three-flavor oscillations occur, the linearity correction 
increases the shower energy by approximately $1.5 \%$. A conservative contribution to the relative energy scale uncertainty is chosen at both detectors with $20 \% \times 1.5 \%=0.3 \%$.

There are three sources of error that were considered when determining the ND and FD MEU values: agreement between data and MC simulation, spatial variation, and the differences between the cosmic and beam stopping muon samples. The default MEU calculation utilizes stopping muons extracted from the cosmic muon dataset. The light level is tuned in the cosmic muon $\mathrm{MC}$ sample to give an MEU value similar to that of the data and a systematic uncertainty is assigned based on different techniques to extract the level of agreement between data and simulation.

The calibration procedure was designed to remove spatial and temporal variations in the MEU constants, as is shown in Figures 3.12 and 3.13. The cosmic stopping muon event numbers are large enough that the fiducial volume is divided up to identify spatial biases across the detectors. The fiducial volumes of the ND and FD are broken down into concentric rings of equal fiducial mass centered around the beam spot and in four volumes parallel to the beam to assign a systematic uncertainty.

Finally, the remaining difference in MEU constants obtained from cosmic stopping muons and stopping muons originating from NuMI neutrino events is taken as an additional systematic uncertainty. 
An energy dependent uncertainty on the modeling of stopping muons to obtain the hadronic shower energy scale $[117,130]$ is summed in quadrature with the absolute energy scale uncertainty from the calibration process to obtain an uncertainty on the calorimetric energy that is parametrized as [131]

$$
\sigma_{\text {shower }}=6.6 \%+3.5 \% \times \exp \left[-\frac{E_{\text {reco }}}{1.44 \mathrm{GeV}}\right] \text {, }
$$

with $100 \%$ correlations assumed between energy bins.

\subsubsection{Neutrino Cross Section Uncertainty}

Neutrino cross sections in the MINOS detectors are simulated using the NEUGEN software, as mentioned in Section 3.6. The uncertainties in the $\mathrm{CC}$ and NC neutrino cross sections are evaluated by changing the NEUGEN parameters, which is discussed in more detail in Ref. [45]. Figure 5.9 shows the cross selection uncertainties in the $\mathrm{CC}$ and $\mathrm{NC}$ samples for the Far-over-Near ratio and their corresponding covariance matrices.

\subsubsection{Background Uncertainty}

The uncertainty in the NC background events in the CC sample [132] comprises of a $15 \%$ uncertainty obtained from assessing the accidental CC event reconstruction rate of muon-removed $\mathrm{CC}$ data and $\mathrm{MC}$ events that mimic NC events, and an 11\% uncertainty that leads to the best agreement between data and MC simulation when freely scaling the NC component in the CC sample. 

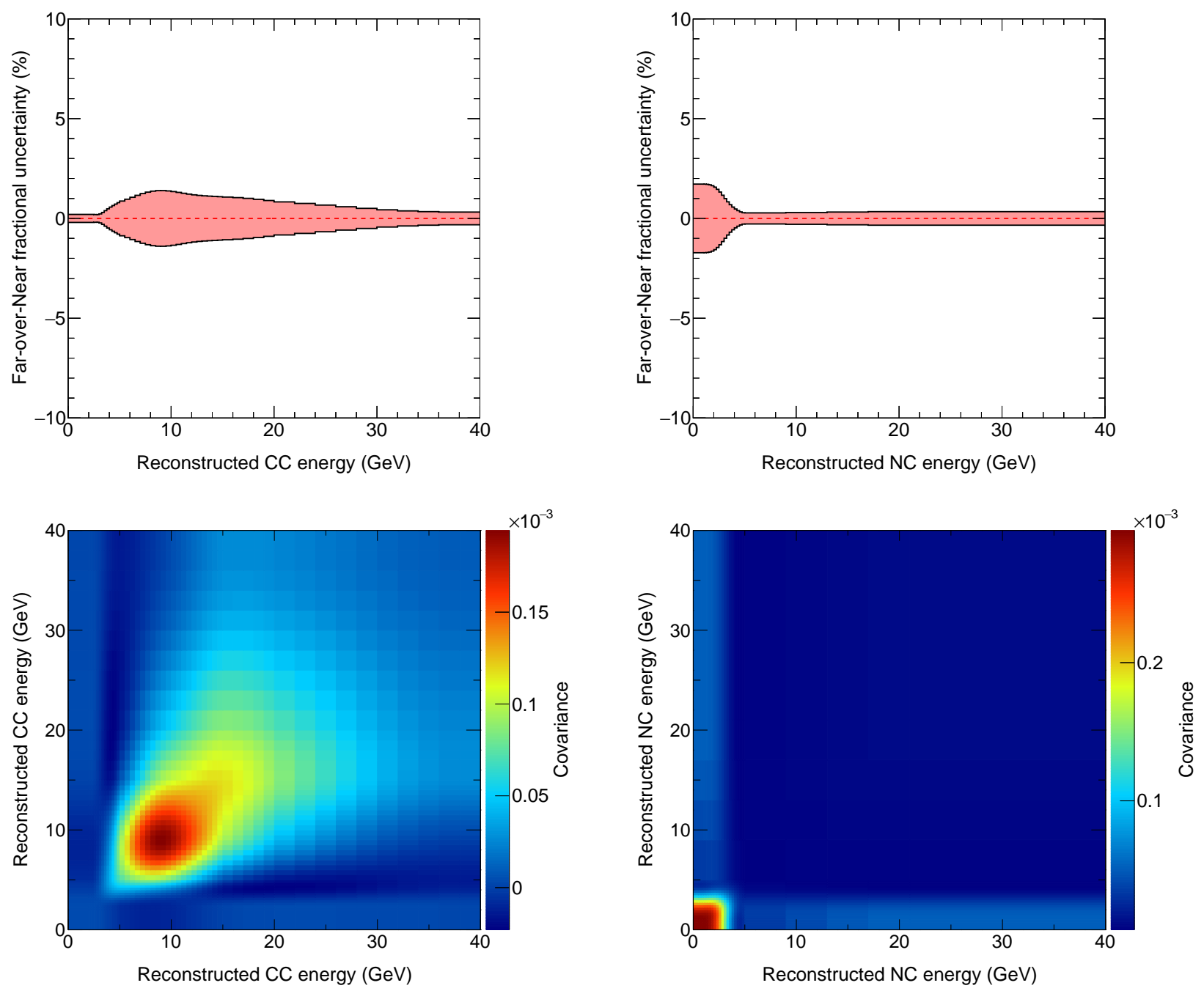

Figure 5.9: The neutrino cross section systematic uncertainty for the MINOS samples. The fractional uncertainty in the $\mathrm{CC}$ (top left) and NC (top right) Far-over-Near ratios and the corresponding covariance matrices for the $\mathrm{CC}$ (bottom left) and $\mathrm{NC}$ (bottom right) samples. 
The uncertainty in the CC background events in the NC sample is evaluated using a direct measurement of the $\mathrm{CC}$ background in the $\mathrm{NC}$ spectrum for different operational configurations of the NuMI beam and results in a $15 \%$ uncertainty [133]. Figure 5.10 shows the NC and CC background uncertainties in the CC and NC samples for the Far-over-Near ratio and their corresponding covariance matrices.

\subsubsection{Hadron Production Uncertainty}

The uncertainty in hadron production simulated by FLUKA, as discussed in Section 3.6, is evaluated in Ref. [134] for MINOS. By varying hadron production parameters [135] within reasonable limits, a set of shifted flux histograms is generated from which a covariance matrix is constructed. Figure 5.11 shows the $\mathrm{CC}$ and $\mathrm{NC}$ hadron production uncertainties for the Farover-Near ratio and the corresponding covariance matrices.

\subsubsection{Beam Optics Uncertainty}

Uncertainties in the simulation of the hadron focusing by the magnetic horns is incorporated in the beam optics uncertainty. In MINOS, uncertainties in the absolute value of the horn current, the horn current distribution, and the magnetic horn position are taken into account [136-138]. Figure 5.12 shows

the $\mathrm{CC}$ and $\mathrm{NC}$ beam optics uncertainties for the Far-over-Near ratio and the corresponding covariance matrices. 

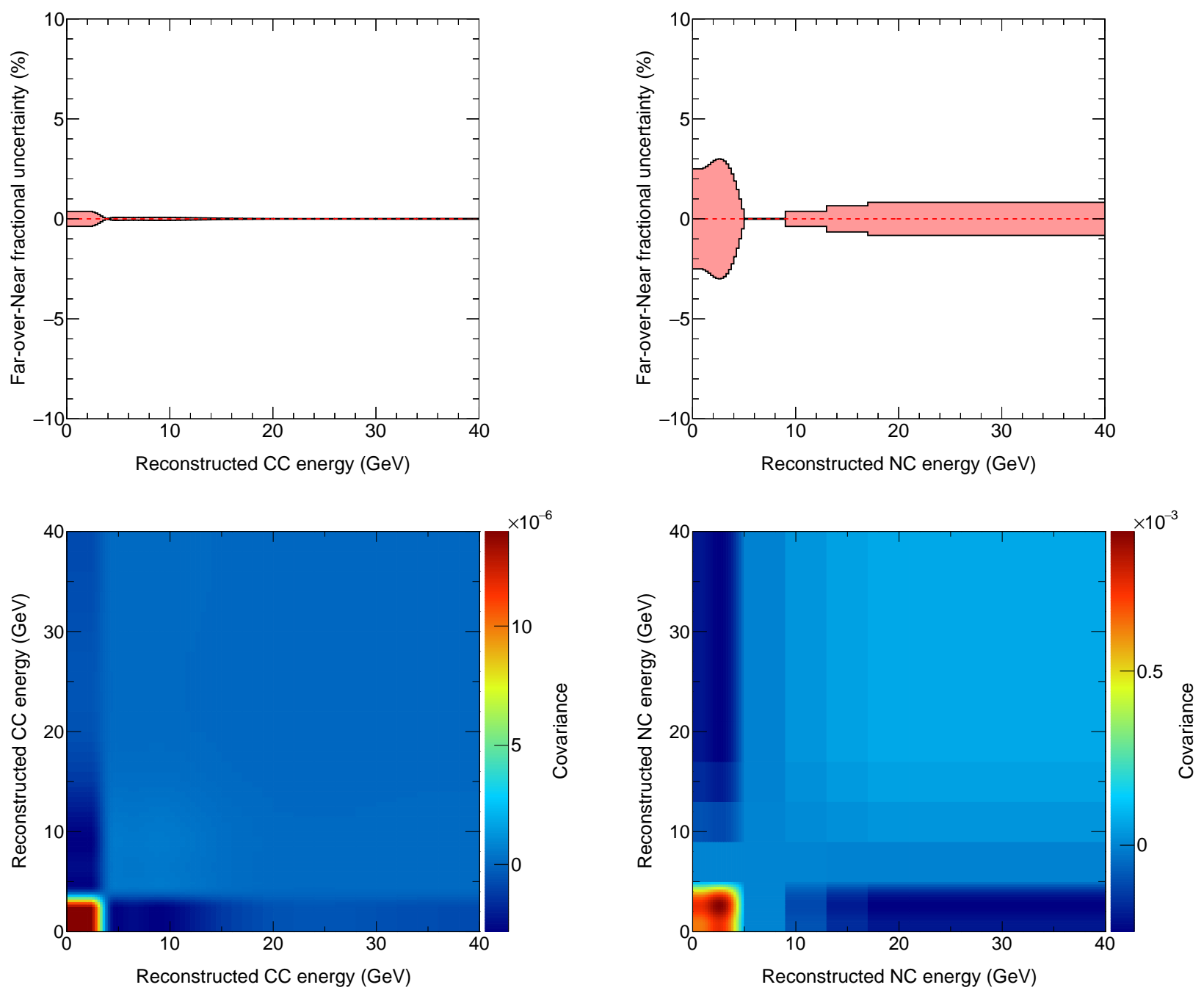

Figure 5.10: The background systematic uncertainty for the MINOS samples. The fractional uncertainty in the CC (top left) and NC (top right) Far-overNear ratios and the corresponding covariance matrices for the CC (bottom left) and $\mathrm{NC}$ (bottom right) samples. 

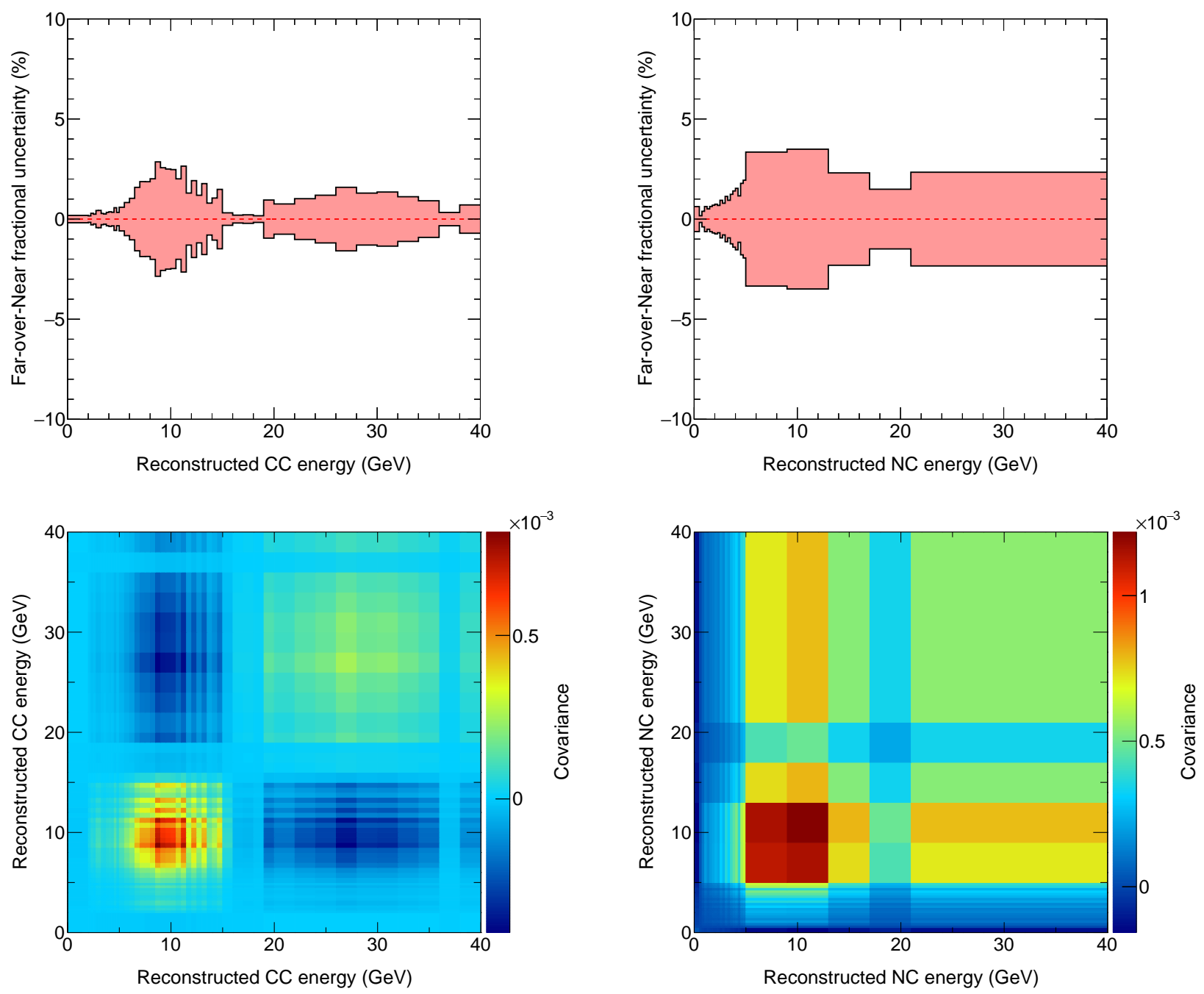

Figure 5.11: The hadron production systematic uncertainty for the MINOS samples. The fractional uncertainty in the CC (top left) and NC (top right) Far-over-Near ratios and the corresponding covariance matrices for the $\mathrm{CC}$ (bottom left) and $\mathrm{NC}$ (bottom right) samples. 

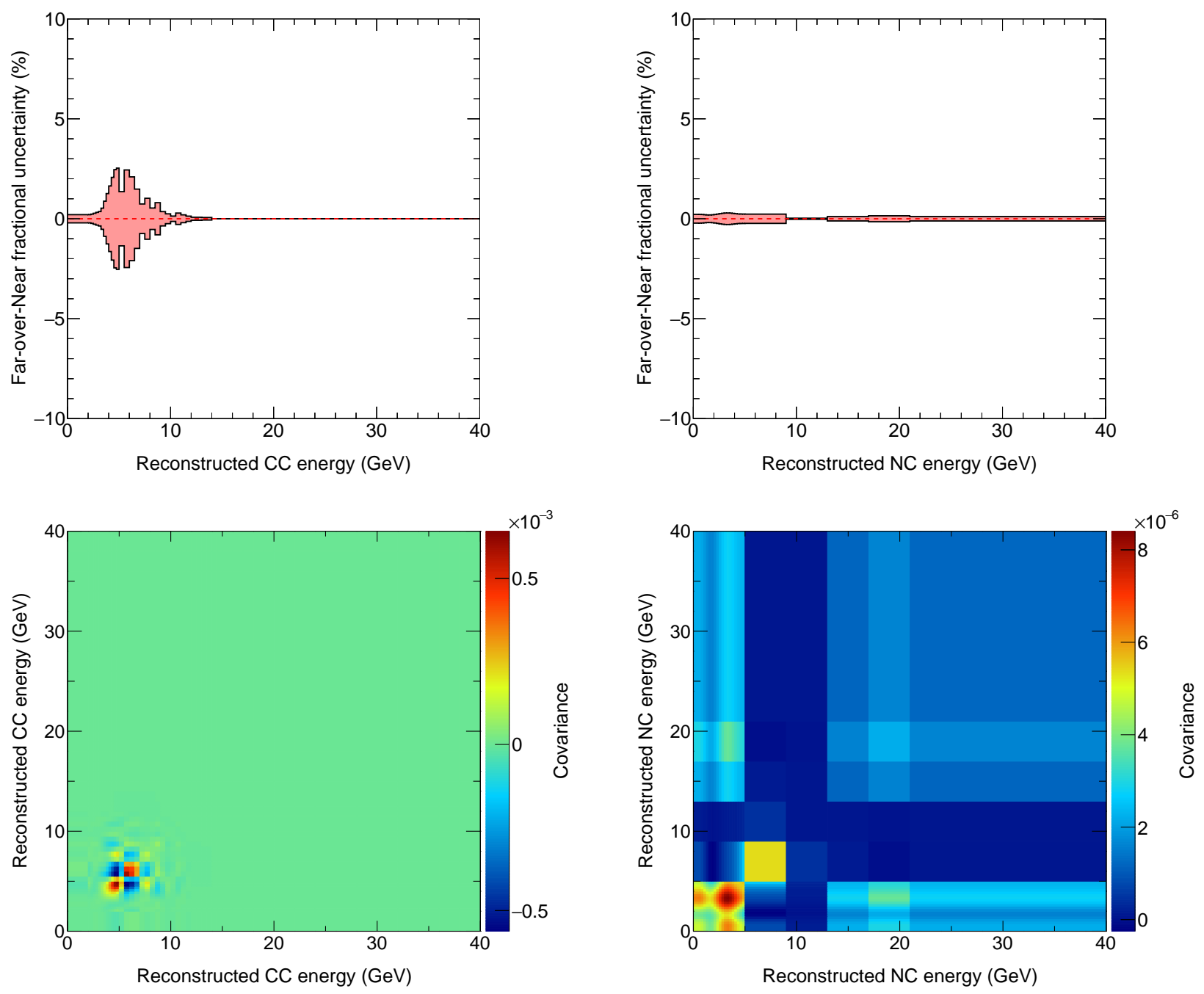

Figure 5.12: The beam optics systematic uncertainty for the MINOS samples. The fractional uncertainty in the CC (top left) and NC (top right) Far-overNear ratios and the corresponding covariance matrices for the CC (bottom left) and NC (bottom right) samples. 


\subsection{Sensitivity and Data $\chi^{2}$ Surfaces}

The $\chi^{2}$ surfaces for the MINOS Far-over-Near Asimov sensitivity are shown in Fig. 5.13. The Asimov sensitivity is obtained from comparing the Far-over-Near LED model predictions with simulated three-flavor data, shown in Fig. 4.10 and Fig. 5.1, corresponding to the MINOS exposure of $10.56 \times 10^{20}$ POT. The values for the three-flavor parameters used to generate the simulated three-flavor data are listed in Table 5.5. A very flat $\chi^{2}$ surface, with a shallow global minimum, is obtained for most of the $\left(R, m_{0}\right)$ plane where $R \lesssim 10^{-7} \mathrm{~m}$, as is shown in the top left panel of Fig. 5.13. The $\Delta \chi^{2}=\chi^{2}-\chi_{\min }^{2}$ surface is shown in the top right panel, where $\chi_{\min }^{2}$ is the global minimum in the $\chi^{2}$ plane. The bottom panels of Fig. 5.13 show the values of the atmospheric parameters that minimize the $\chi^{2}$ test statistic at a fixed point in the $\left(R, m_{0}\right)$ plane. The white region in the bottom left panel corresponds to $\Delta m_{32}^{2}$ values between $10^{-6}$ and $1.4 \times 10^{-3} \mathrm{eV}^{2}$. The fitting procedure obtains atmospheric parameter values that are consistent with the three-flavor simulated data in the region of the $\left(R, m_{0}\right)$ plane that corresponds to a very flat $\chi^{2}$ surface, whereas significantly different values are obtained in regions where the LED model predicts oscillations inconsistent with the three-flavor paradigm, in particular where oscillations along the ND baseline are predicted.

The same $\chi^{2}$ surfaces are shown in Fig. 5.14 for the MINOS Far-overNear data. A shallow global minimum is found at $R=0.035 \mu \mathrm{m}, m_{0}=0.941 \mathrm{eV}$, $\Delta m_{32}^{2}=2.78 \times 10^{-3} \mathrm{eV}^{2}$, and $\sin ^{2} \theta_{23}=0.612$. Figure 5.1 compares the global minimum LED prediction to the data. The three-flavor prediction and the 


\begin{tabular}{|c|c|}
\hline Parameter & Value \\
\hline \hline$\Delta m_{32}^{2}$ & $2.37 \times 10^{-} 3 \mathrm{eV}^{2}$ \\
$\Delta m_{21}^{2}$ & $7.54 \times 10^{-5} \mathrm{eV}^{2}$ \\
$\theta_{23}$ & 0.695 \\
$\theta_{13}$ & 0.149 \\
$\theta_{12}$ & 0.588 \\
$\delta_{\mathrm{CP}}$ & 0 \\
\hline
\end{tabular}

Table 5.5: The three-flavor oscillation parameter values used to generate the three-flavor simulated data.

lowest $\chi^{2}$ LED prediction are almost identical and the data is consistent with the three-flavor paradigm. The next section discusses the interpretation of the sensitivity and data $\chi^{2}$ surfaces.

\subsection{Feldman-Cousins Data Limit}

For $\alpha$ parameters or degrees of freedom, a confidence region for a given confidence level (C.L.) corresponding to $N$ standard deviations is constructed using

$$
\chi^{2}(\hat{\boldsymbol{\theta}} \pm N \hat{\boldsymbol{\sigma}})=\chi_{\min }^{2}+\Delta \chi_{\alpha}^{2}(\text { C.L. })
$$

where $\hat{\boldsymbol{\theta}}$ is the set of parameter values that minimizes the $\chi^{2}$ test statistic.

Table 5.6 lists the values for a few cases. Two common ways to interpret a two-dimensional surface, such as the $\left(R, m_{0}\right)$ plane, are the global and raster scan. The former defines a confidence region around the global minimum, $\chi_{\min }^{2}$, defined by $\Delta \chi_{2}^{2}$ (C.L.). The latter uses for a fixed value of one parameter, e.g. $m_{0}$, the local minimum for the second parameter, with 

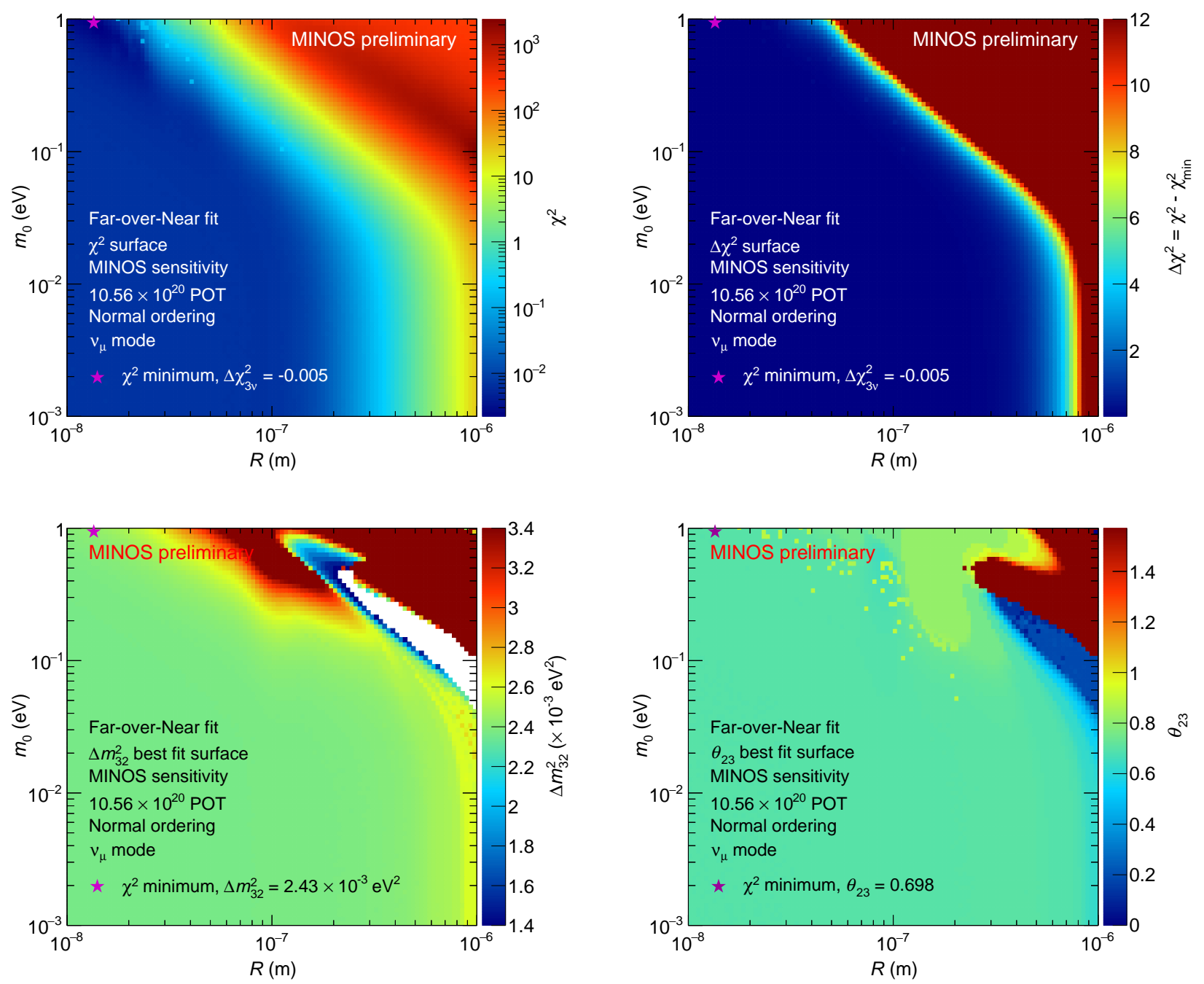

Figure 5.13: The sensitivity surfaces for the LED model in the $\left(R, m_{0}\right)$ plane for a simulated MINOS exposure of $10.56 \times 10^{20}$ POT and assuming threeflavor simulated data: the $\chi^{2}$ surface (top left), the $\Delta \chi^{2}$ surface (top right), and the best fit values for $\Delta m_{32}^{2}$ (bottom left) and $\theta_{23}$ (bottom right). The white region in the bottom left panel corresponds to $\Delta m_{32}^{2}$ values between $10^{-6}$ and $1.4 \times 10^{-3} \mathrm{eV}^{2}$. 

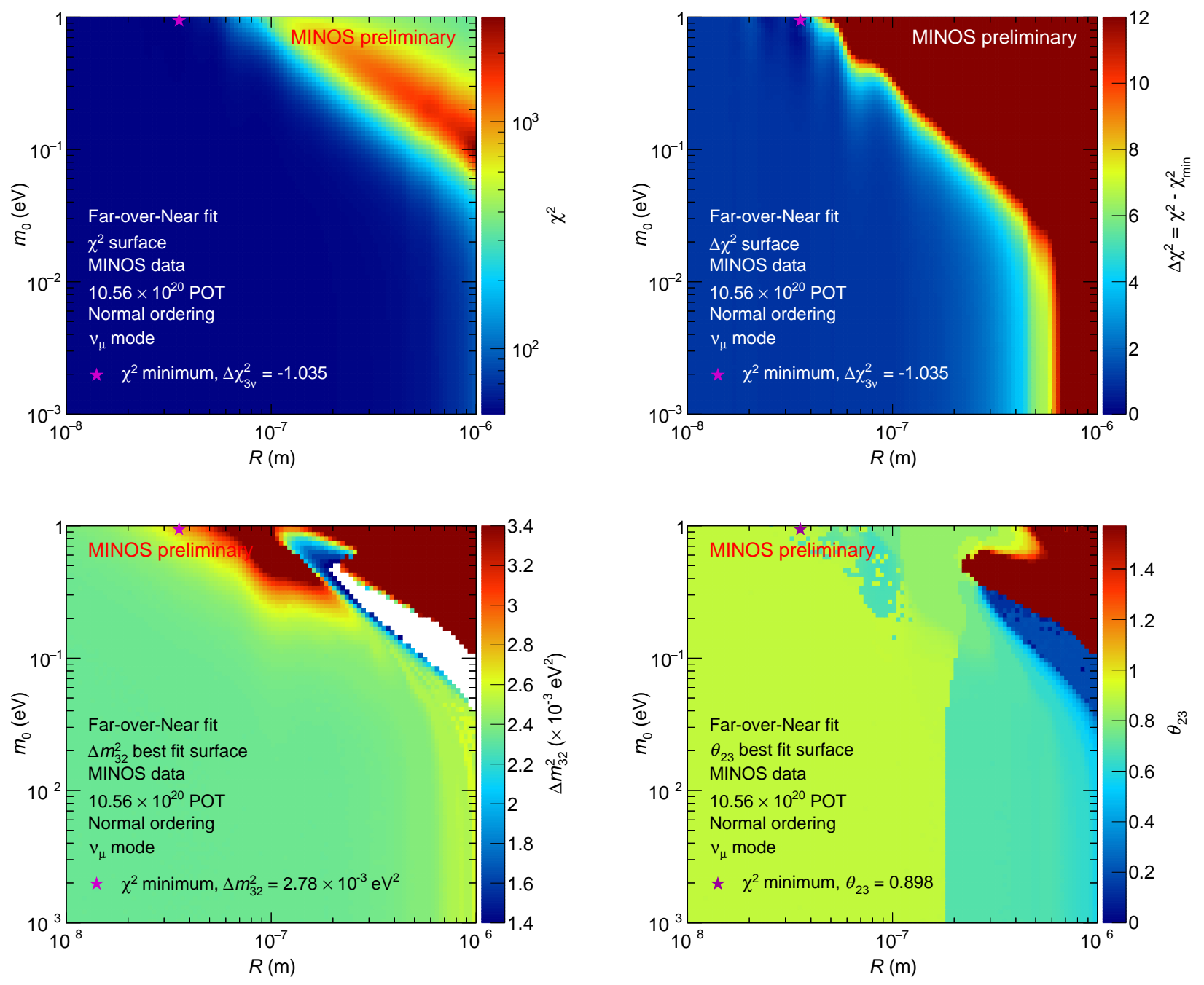

Figure 5.14: The data surfaces for the LED model in the $\left(R, m_{0}\right)$ plane for the MINOS exposure of $10.56 \times 10^{20}$ POT: the $\chi^{2}$ surface (top left), the $\Delta \chi^{2}$ surface (top right), and the best fit values for $\Delta m_{32}^{2}$ (bottom left) and $\theta_{23}$ (bottom right). The white region in the bottom left panel corresponds to $\Delta m_{32}^{2}$ values between $10^{-6}$ and $1.4 \times 10^{-3} \mathrm{eV}^{2}$. 


\begin{tabular}{|c|c|c|c|c|}
\hline & $68 \%$ & $90 \%$ & $95 \%$ & $99 \%$ \\
$\Delta \chi_{\alpha}^{2}$ & $1 \sigma$ & $1.64 \sigma$ & $1.96 \sigma$ & $2.58 \sigma$ \\
\hline \hline$\Delta \chi_{1}^{2}$ & 1.00 & 2.71 & 3.84 & 6.63 \\
$\Delta \chi_{2}^{2}$ & 2.30 & 4.61 & 5.99 & 9.21 \\
$\Delta \chi_{3}^{2}$ & 3.53 & 6.25 & 7.81 & 11.34 \\
$\Delta \chi_{4}^{2}$ & 4.72 & 7.78 & 9.49 & 13.28 \\
\hline
\end{tabular}

Table 5.6: The $\Delta \chi^{2}=\chi^{2}-\chi_{\min }^{2}$ values for the $68 \%, 90 \%, 95 \%$, and $95 \%$ confidence levels (C.L.) for 1 to 4 degrees of freedom $(\alpha)$. The P-value is given by 1-C.L.(\%).

$\Delta \chi_{1}^{2}$ (C.L.). Figure 5.15 illustrates the difference between the global and raster scan in a simple two-dimensional toy $\chi^{2}$ surface.

The correct use of Formula (5.22) and Table 5.6 requires that the measurements are distributed according to a Gaussian distribution around the true parameter values. It is shown in Ref. [45] and Section 5.4.2 that this is not a valid assumption for the MINOS Far-over-Near LED analysis. A global or raster scan will lead to an incorrect coverage (either more or less) when constructing a confidence region.

The Feldman-Cousins (FC) technique [139] ensures an exact coverage when constructing a confidence region. This technique uses a set of fluctuated simulated data at each point in the two-dimensional plane to determine the correct $\Delta \chi_{2}^{2}$ (C.L.) value for that point. The FC confidence region is then outlined by those points in the two-dimensional plane where $\Delta \chi^{2}=\chi^{2}-\chi_{\min }^{2}=\Delta \chi_{2, \mathrm{FC}}^{2}($ C.L. $)$. When $\Delta \chi_{2, \mathrm{FC}}^{2}($ C.L. $) \neq 4.61$, a confidence region should be constructed employing the FC technique to ensure exact coverage. Section 5.4.2 discusses the FC study for the MINOS Far-over-Near LED 


$$
\Delta \chi_{\text {global }}^{2}=\chi_{\text {raw }}^{2}-\underset{\text { min }}{2}
$$

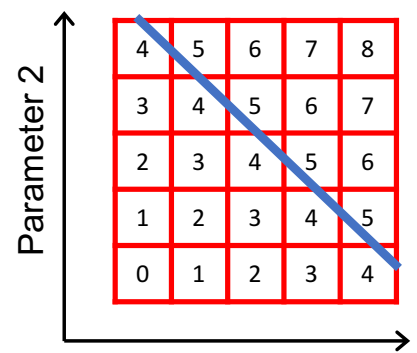

$\Delta \chi_{\text {global }}^{2}$

Parameter 1

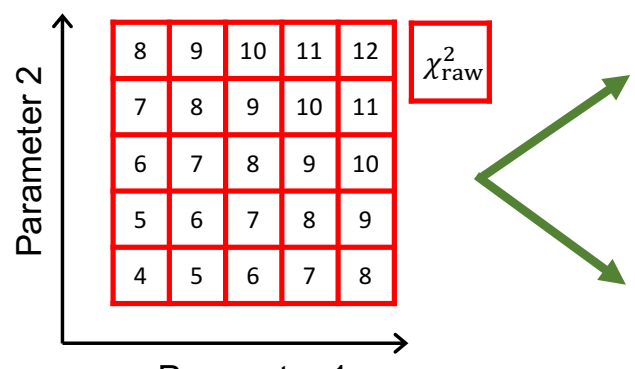

Parameter 1

$$
\Delta \chi_{\text {raster }}^{2}=\chi_{\text {raw }}^{2}-\chi_{\text {min }}^{2}
$$

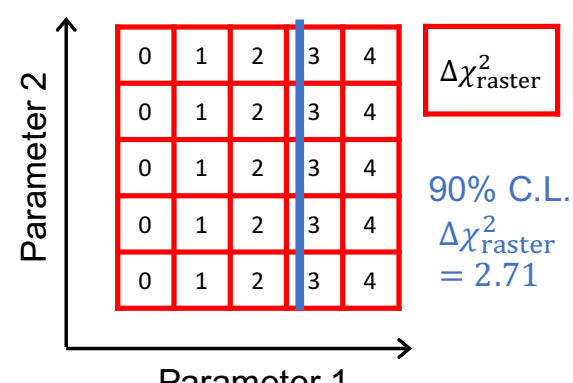

Parameter 1

Figure 5.15: Illustration of the difference between the global scan (top right) and raster scan (bottom right) 90\% C.L. limits for a simple two-dimensional toy $\chi^{2}$ surface (left). 
analysis in more detail. Figure B.8 in Appendix B demonstrates in the context of a $3+1$ sterile neutrino model how at a fixed row in the $\Delta \chi^{2}$ plane, the FC coverage can be obtained from the $\Delta \chi_{2, \mathrm{FC}}^{2}$ (C.L.) profile.

As discussed in References [139] and [46], an FC study is generally advised to ensure correct coverage. The reason for an incorrect coverage could be

- the presence of non-Gaussian distributions within the analysis, e.g. the sinusoidal nature of the neutrino oscillation probabilities may cause the fitting procedure, for which the $\chi^{2}$ distribution assumes a Gaussian probability density function, to find a global or local minimum away from the true parameter values for a set of fluctuated simulated data;

- the presence of best fit points near or in an unphysical region of the parameter space; and

- for a given dataset, the choice between constructing a one-sided (open) confidence region for a null result or a two-sided (closed) confidence region for a non-null result.

The final results presented in this dissertation are obtained through an FC interpretation of the $\Delta \chi^{2}$ surface. 


\subsubsection{Interpreting the Sensitivity and Data $\chi^{2}$ Surfaces}

Figure 5.16 shows the $90 \%$ C.L. Asimov sensitivity and data coverage obtained using a global scan with $\Delta \chi^{2}=4.61$ (top row) and the $90 \%$ C.L. data coverage obtained using the FC technique.

The top left panel of Fig. 5.16 shows the cumulative effect on the global 90\% C.L. Asimov sensitivity coverage of adding the systematic uncertainties to the statistical uncertainties in the covariance matrix.

The top right panel of Fig. 5.16 compares the global 90\% C.L Asimov sensitivity and data coverages. The difference between the Asimov sensitivity and the data coverage, in particular at smaller $m_{0}$ values, is investigated in Section 5.4.3.

The bottom left panel of Fig. 5.16 compares the raster scan $\left(\Delta \chi_{1}^{2}=\right.$ 2.71), global scan $\left(\Delta \chi_{2}^{2}=4.61\right)$, and FC $90 \%$ C.L data coverages. Only small differences are observed between the three methods. The FC study used to obtain the FC data coverage is discussed in Section 5.4.2. In the limit of a vanishing lightest neutrino mass, the large extra dimension size is constrained to be smaller than $0.45 \mu \mathrm{m}$ at $90 \%$ C.L. by the MINOS Far-over-Near analysis. The FC $90 \%$ C.L data coverage is overlaid on the $\sin ^{2} \theta_{\text {eff }}^{\mu \mu}$ surface, as defined in Formula (2.34), in the bottom right panel of Fig. 5.16. For $m_{0} \lesssim 0.1 \mathrm{eV}$, mixing effects due to the existence of large extra dimensions that are larger than about $6 \%$ are excluded at $90 \%$ C.L. by the MINOS Far-over-Near data. 
The two different behaviors in the $\left(R, m_{0}\right)$ plane displayed by the confidence limits in Fig. 5.16 can be understood qualitatively. For $m_{0} \gtrsim 0.05 \mathrm{eV}$, the neutrino masses become degenerate (see for example Fig. 2.15) and the limit depends on $\xi=\xi_{1}=\xi_{2}=\xi_{3}=\sqrt{2} m_{0} R$ and follows the $\xi$ contours shown in Fig. 2.12. It should be noted that the linear behavior of the confidence limit at high $m_{0}$ values because of the dependence on $\xi$ is easier to observe when a logarithmic scale is used for the horizontal axis (see for example Fig. 5.20). For $m_{0} \ll 0.05 \mathrm{eV}$, the model is predominantly constrained by $\xi_{3}$ when assuming normal ordering, as shown in Formula (2.21), such that the confidence limit does not depend on $m_{0}$.

\subsubsection{Feldman-Cousins Study}

Figure 5.17 shows the $90 \%$ up value surface of the $\Delta \chi_{\mathrm{FC}}^{2}$ distributions used to construct the MINOS Far-over-Near FC 90\% C.L. data coverage in Fig. 5.16. The $90 \%$ up value at a fixed point in the $\left(R, m_{0}\right)$ plane is obtained from the $\Delta \chi_{\mathrm{FC}}^{2}$ distribution for a set of fluctuated simulated data spectra with true parameter values at that point, for which

$$
\Delta \chi_{\mathrm{FC}}^{2}=\chi_{\text {profile }}^{2}-\chi_{\text {best }}^{2}
$$

where $\chi_{\text {profile }}^{2}$ and $\chi_{\text {best }}^{2}$ are the $\chi^{2}$ minima obtained from a fit to the fluctuated simulated dataset with fixed $R$ and $m_{0}$ and with $R$ and $m_{0}$ allowed to vary freely, respectively. The atmospheric parameters $\Delta m_{32}^{2}$ and $\theta_{23}$ are free to vary in the fit in both cases. In the Far-over-Near analysis, the best fit values for $\Delta m_{32}^{2}$ and $\theta_{23}$ obtained from the data fit (shown in Fig. 5.14) are adopted as 

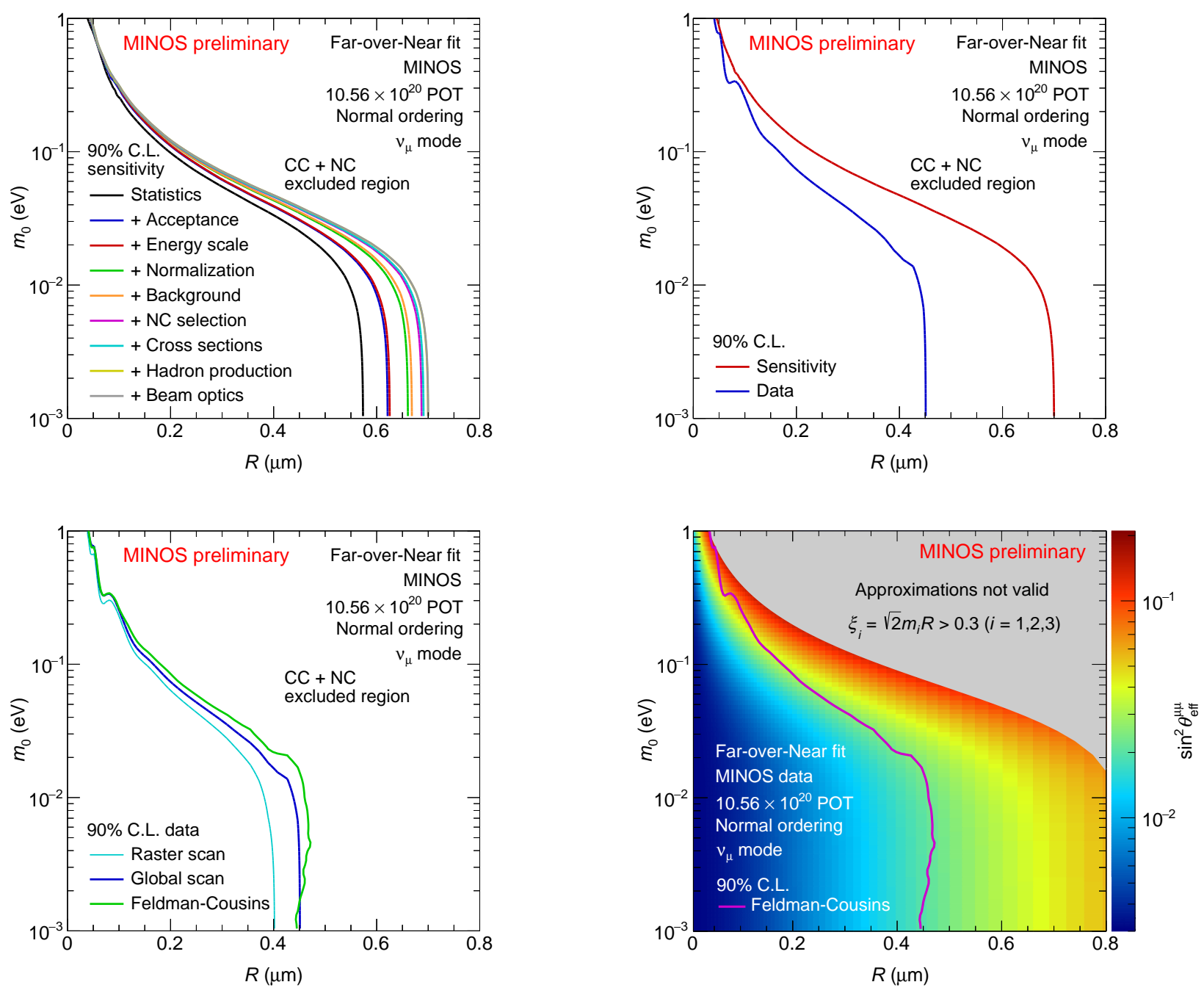

Figure 5.16: Interpreting the sensitivity and data $\chi^{2}$ surfaces for the MINOS exposure of $10.56 \times 10^{20}$ POT: the cumulative effect of the systematic uncertainties on the global $90 \%$ C.L. $\left(\Delta \chi^{2}=4.61\right)$ Asimov sensitivity coverage (top left), comparing the global $90 \%$ C.L. $\left(\Delta \chi^{2}=4.61\right)$ Asimov sensitivity and data coverage (top right), and the Feldman-Cousins $90 \%$ C.L. data coverage (bottom left) and its interpretation in the $\sin ^{2} \theta_{\text {eff }}^{\mu \mu}$ plane (bottom right). 
true values for the simulated data. A different approach that does not rely on data is used for the FC study in the two-detector analysis in Chapter 6 .

A statistically and systematically fluctuated simulated dataset is obtained using the covariance matrix $V$. At a given point in the $\left(R, m_{0}\right)$ plane, after dynamic scaling, the covariance matrix is decomposed in an upper triangular matrix $L$ and its transpose, such that

$$
V=L^{\top} L
$$

The LED Far-over-Near prediction at the point, $\mathbf{R}_{\mathrm{LED}}$, is used as nominal simulated data. A set of random numbers $\mathbf{g}$ drawn from a Gaussian distribution with $\mu=0$ and $\sigma=1$, one for every bin in $\mathbf{R}_{\mathrm{LED}}$, is transformed into a set of correlated numbers using the triangular matrix $L$, such that the fluctuated simulated data is given by

$$
\mathbf{R}_{\mathrm{LED}}^{\mathrm{fluc}}=L \mathbf{g}+\mathbf{R}_{\mathrm{LED}}
$$

A validation study of the fluctuation procedure (in the context of the two-detector analysis presented in Chapter 6) is discussed in Appendix A.

Figures 5.18 and 5.19 show the distributions for the Far-over-Near FC study at two different points in the $\left(R, m_{0}\right)$ plane. The top left figure shows the $\Delta \chi_{\mathrm{FC}}^{2}$ distribution for 500 fluctuated simulated experiments. The green dashed line indicates the $90 \%$ up value for $\Delta \chi_{\mathrm{FC}}^{2}$, meaning that 450 of the fluctuated experiments have a smaller $\Delta \chi_{\mathrm{FC}}^{2}$ and 50 fluctuated experiments have a larger $\Delta \chi_{\mathrm{FC}}^{2}$. 
The $\Delta \chi_{\mathrm{FC}}^{2}$ distribution in Fig. 5.18 looks like a typical $\chi^{2}$ distribution for two degrees of freedom and has a $90 \%$ up value of 4.60, which agrees well with $\Delta \chi_{2}^{2}(90 \%$ C.L. $)=4.61$ used for a global scan. The top right panel in Fig. 5.18 shows the $\chi_{\text {profile }}^{2}$ and $\chi_{\text {best }}^{2}$ distributions used in Formula (5.23). The middle and bottom row panels show the best fit value distributions of $R, m_{0}$, $\Delta m_{32}^{2}$, and $\theta_{23}$ for the simulated experiments at this point. The $R_{\text {best }}, m_{0, \text { best }}$, and $\Delta m_{32 \text {,best }}^{2}$ distributions are approximately Gaussian around the true values (indicated by the dashed blue lines), while the $\theta_{23 \text {, best }}$ distribution displays the ambiguities of the lower and upper octant.

The $\Delta \chi_{\mathrm{FC}}^{2}$ distribution in Fig. 5.19 has a $90 \%$ up value of 5.31, which is significantly different from the Gaussian assumption. To ensure a correct $90 \%$ C.L. coverage, $\Delta \chi_{\mathrm{FC}}^{2}=5.31$ should be employed at this point in the $\left(R, m_{0}\right)$ plane. The $R_{\text {best }}$ and $m_{0, \text { best }}$ distributions are not Gaussian. In particular, the $m_{0 \text {,best }}$ values are not centered around the true $m_{0}$ value. Note that this indicates that the fitter has no sensitivity to $m_{0}$ at this point in the $\left(R, m_{0}\right)$ plane.

An FC study is CPU intensive and, because of that, is not performed at every point in the $\left(R, m_{0}\right)$ plane. It is only required to know $\Delta \chi_{\mathrm{FC}}^{2}$ where the confidence region is likely to be. As such, the global data coverage is used to identify a selected number of points where an FC study is needed. A more comprehensive FC study is performed for the two-detector analysis presented in Chapter 6. 


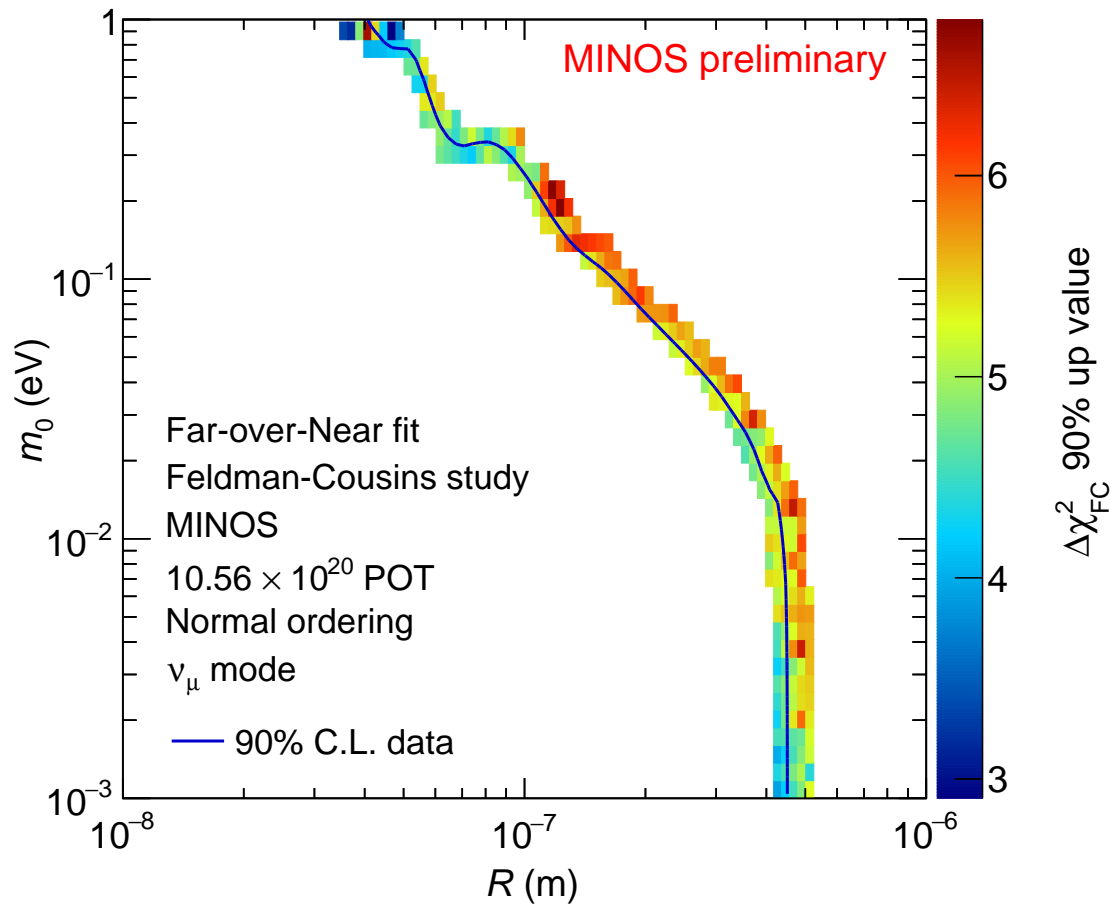

Figure 5.17: The $90 \%$ up value surface of the $\Delta \chi_{\mathrm{FC}}^{2}$ distributions used to construct the MINOS Far-over-Near Feldman-Cousins $90 \%$ C.L. data coverage in Fig. 5.16. The global 90\% C.L. data coverage (blue line) is shown for reference. 

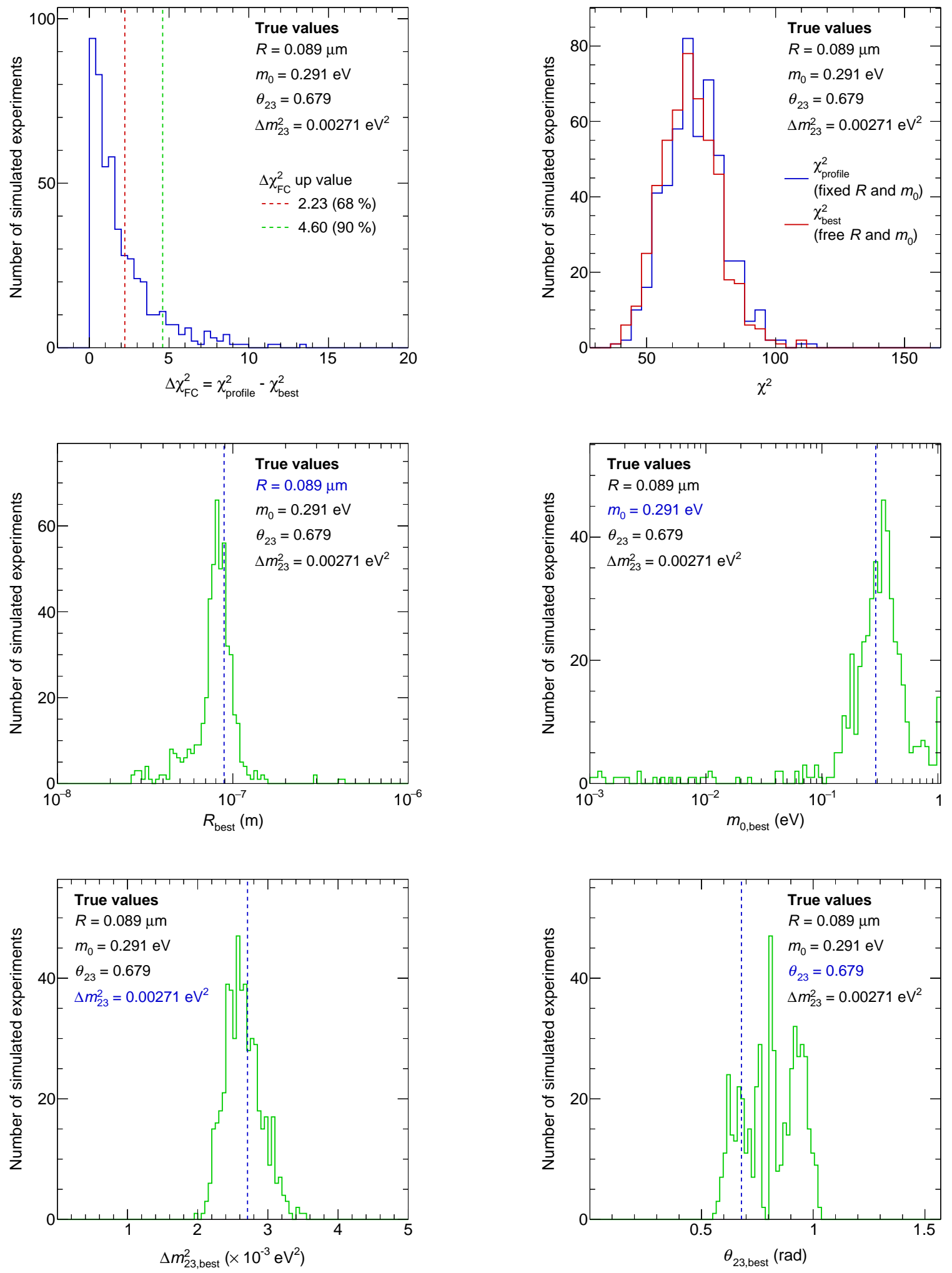

Figure 5.18: The $\Delta \chi_{\mathrm{FC}}^{2}$ and $\chi^{2}$ profiles (top rows) and the best fit distributions (middle and bottom rows) of the LED model parameters for 500 fluctuated simulated experiments assuming the true values $R=0.089 \mu \mathrm{m}, m_{0}=0.291 \mathrm{eV}$, $\Delta m_{32}^{2}=2.71 \times 10^{-3} \mathrm{eV}^{2}$, and $\theta_{23}=0.679$. 

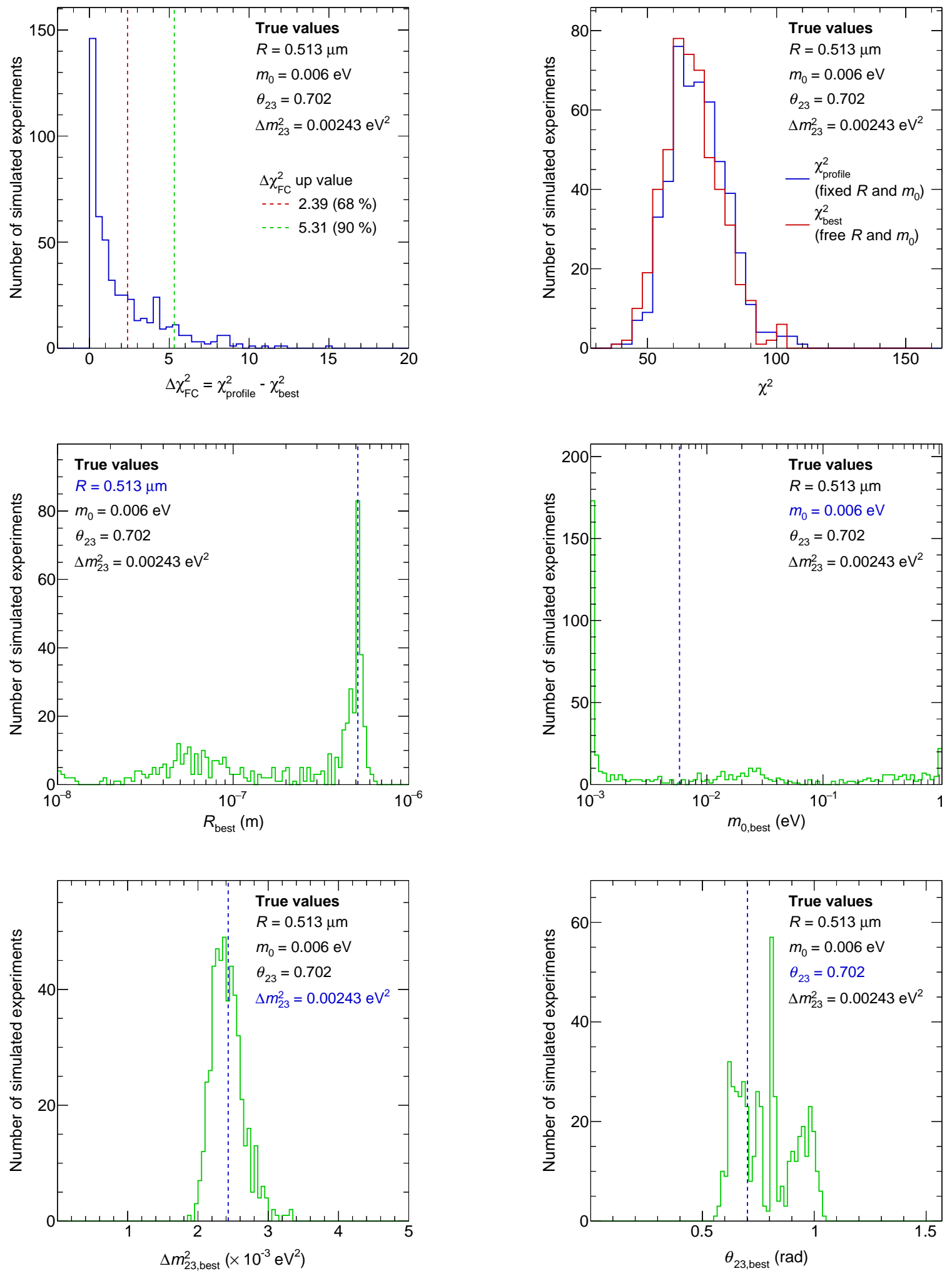

Figure 5.19: The $\Delta \chi_{\mathrm{FC}}^{2}$ and $\chi^{2}$ profiles (top rows) and the best fit distributions (middle and bottom rows) of the LED model parameters for 500 fluctuated simulated experiments assuming the true values $R=0.513 \mu \mathrm{m}, m_{0}=0.006 \mathrm{eV}$, $\Delta m_{32}^{2}=2.43 \times 10^{-3} \mathrm{eV}^{2}$, and $\theta_{23}=0.702$. 


\subsubsection{The Asimov and Median Sensitivity}

The global 90\% C.L. data coverage is more constraining than the global 90\% C.L. Asimov sensitivity coverage, as is shown in Figure 5.16. A study of 1000 statistically and systematically fluctuated simulated three-flavor experiments shows that this is not an unexpected result. In this study, the atmospheric parameters are fixed to the three-flavor values in Table. 5.5 to reduce the computing time of the 1000 sensitivity $\chi^{2}$ surfaces.

The top panel of Fig. 5.20 shows that the effect of fixing the atmospheric parameters on the global 90\% C.L. Asimov sensitivity and data coverages is small. The fluctuated simulated data spectra are obtained employing the same procedure as outlined in Section 5.4.2 for the FC study. The bottom panel of Fig. 5.20 shows the 1000 global 90\% C.L. fluctuated sensitivity coverages. At $m_{0}=0.005 \mathrm{eV}, 39 \%$ of the fluctuated experiments have a stronger exclusion than the data.

As discussed in more detail in Chapter 6 and Appendix B, a comparison of the data coverage to the median of a set of fluctuated sensitivity coverages rather than to the Asimov sensitivity coverage is deemed more representative. However, the Asimov sensitivity coverage remains useful as it allows a quick estimate of the sensitivity. Figure 5.20 shows that the median of the fluctuated sensitivity coverages is closer to the data coverage than the Asimov sensitivity coverage. 

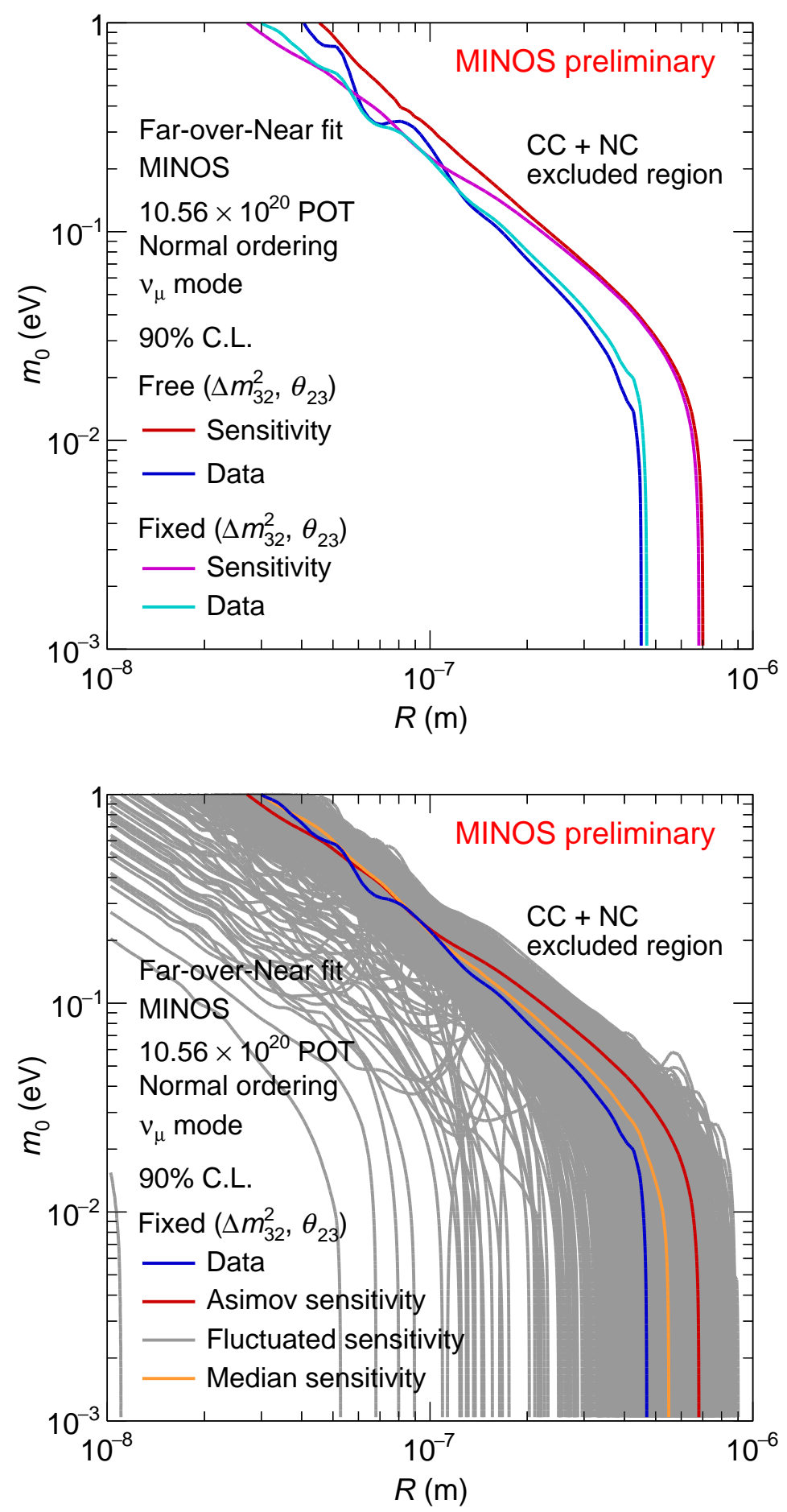

Figure 5.20: A fluctuated sensitivity study. (Top) The effect of the atmospheric parameters $\Delta m_{32}^{2}$ and $\theta_{23}$ on the MINOS Far-over-Near global $90 \%$ C.L. Asimov sensitivity and data coverages. (Bottom) A comparison of the MINOS Far-over-Near global 90\% C.L. Asimov and median fluctuated sensitivity coverages, assuming fixed atmospheric parameters. 


\subsection{Including MINOS+ Data}

A first look at the first two years of MINOS+ data in the context of the Far-over-Near analysis is outlined in this section. The event selection for the first two years of MINOS+ data is discussed in Chapter 4. The addition of MINOS+ data significantly increases the number of neutrino events at higher energies where effects of the LED model are larger, as shown in Chapter 2, and is expected to improve the sensitivity to the LED model of the Far-over-Near analysis.

The analysis strategy is the same as the one discussed in Section 5.1. A slightly different binning scheme is used for the CC and NC samples, as illustrated in Figure 5.21. A new library of LED predictions for the combined MINOS/MINOS+ samples is calculated once at the start of the analysis and is called upon by the fitter.

The systematic uncertainties discussed in Section 5.2 are reassessed for the combined MINOS/MINOS+ samples, with one added systematic uncertainty, the flux modification uncertainty, shown in Fig. 5.22. As mentioned in Section 3.6, the MINOS and MINOS+ neutrino flux prediction is provided by the FLUGG software package, with APS weights applied to provide corrections based on monitoring of NuMI beam conditions. An additional neutrino flux systematic uncertainty is assigned based on the difference between the APS-weighted FLUGG prediction and a neutrino flux prediction provided by the GEANT4-based G4-NuMI software package [91], which is consistent with data collected by the NA49 experiment and has no dependency on NuMI 


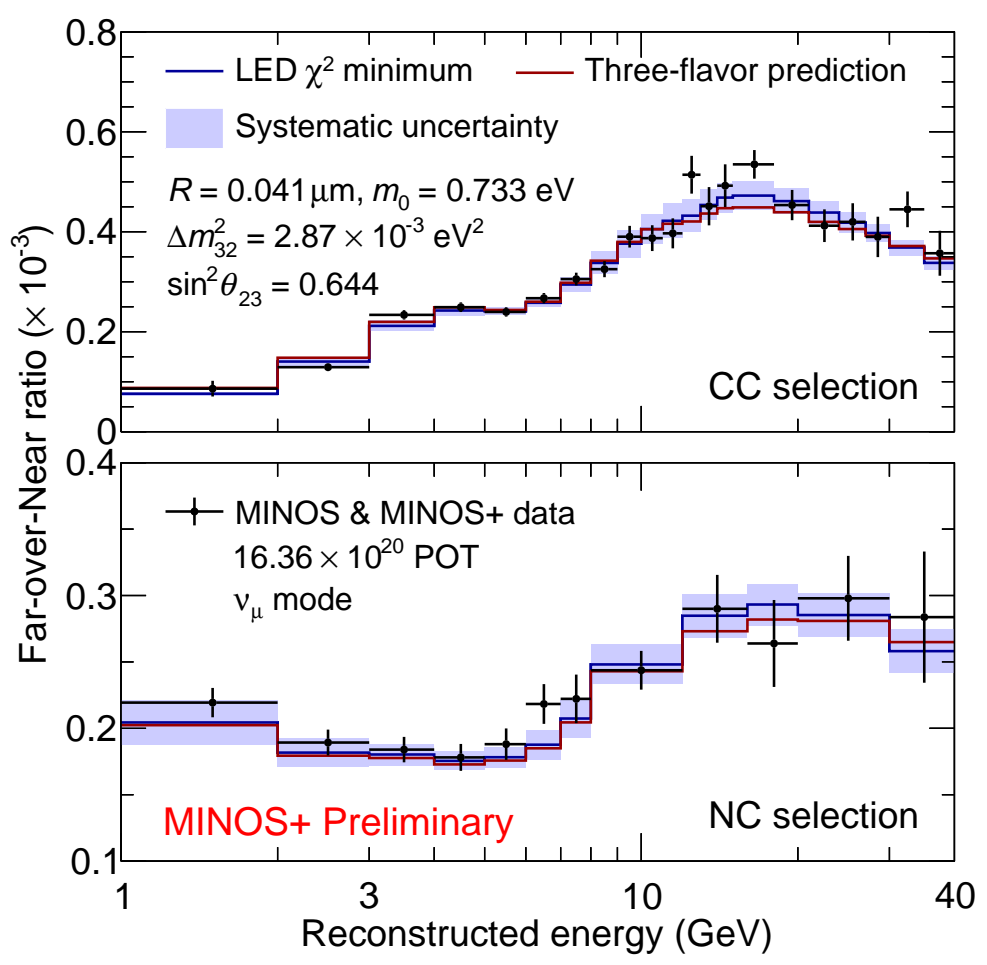

Figure 5.21: The Far-over-Near ratio of the MINOS and MINOS+ CC (top) and NC (bottom) data (black points) as a function of the reconstructed neutrino energy. The error bars represent statistical uncertainties. The threeflavor prediction is shown in red. The LED lowest $\chi^{2}$ prediction is shown with its systematic uncertainty (blue line and band), constructed, for illustration purposes, from the square roots of the diagonal elements of the systematic covariance matrix. 
data. The total systematic uncertainty band and covariance matrices for the MINOS/MINOS+ CC and NC samples are shown in Fig. 5.23.

The $\chi^{2}$ surfaces for the MINOS/MINOS+ Far-over-Near Asimov sensitivity are shown in Fig. 5.24. The three-flavor parameter values listed in Table 5.5 are used to create the simulated Asimov three-flavor data. Figure 5.25 shows the same $\chi^{2}$ surfaces for the MINOS/MINOS+ Far-over-Near data. Figure 5.26 shows the 90\% C.L. Asimov sensitivity and data coverages obtained using a global scan with $\Delta \chi^{2}=4.61$ for the MINOS samples only (also shown in the top right panel of Fig. 5.16 with a linear horizontal axis scale) and for the combined MINOS/MINOS+ samples. The addition of the MINOS+ data in the Far-over-Near analysis strengthens the constraints set on the LED model, with the large extra dimension size $R$ constrained to be smaller than $0.25 \mu \mathrm{m}$ at $90 \%$ C.L. for a vanishing lightest neutrino mass $m_{0}$. It should be noted that this is a global scan rather than an FC interpretation of the $\Delta \chi^{2}$ surface. For various reasons discussed in Chapter 6 , it was decided to move away from the Far-over-Near ratio analysis strategy and instead use the ND and FD CC and NC samples simultaneously in a two-detector fit. 

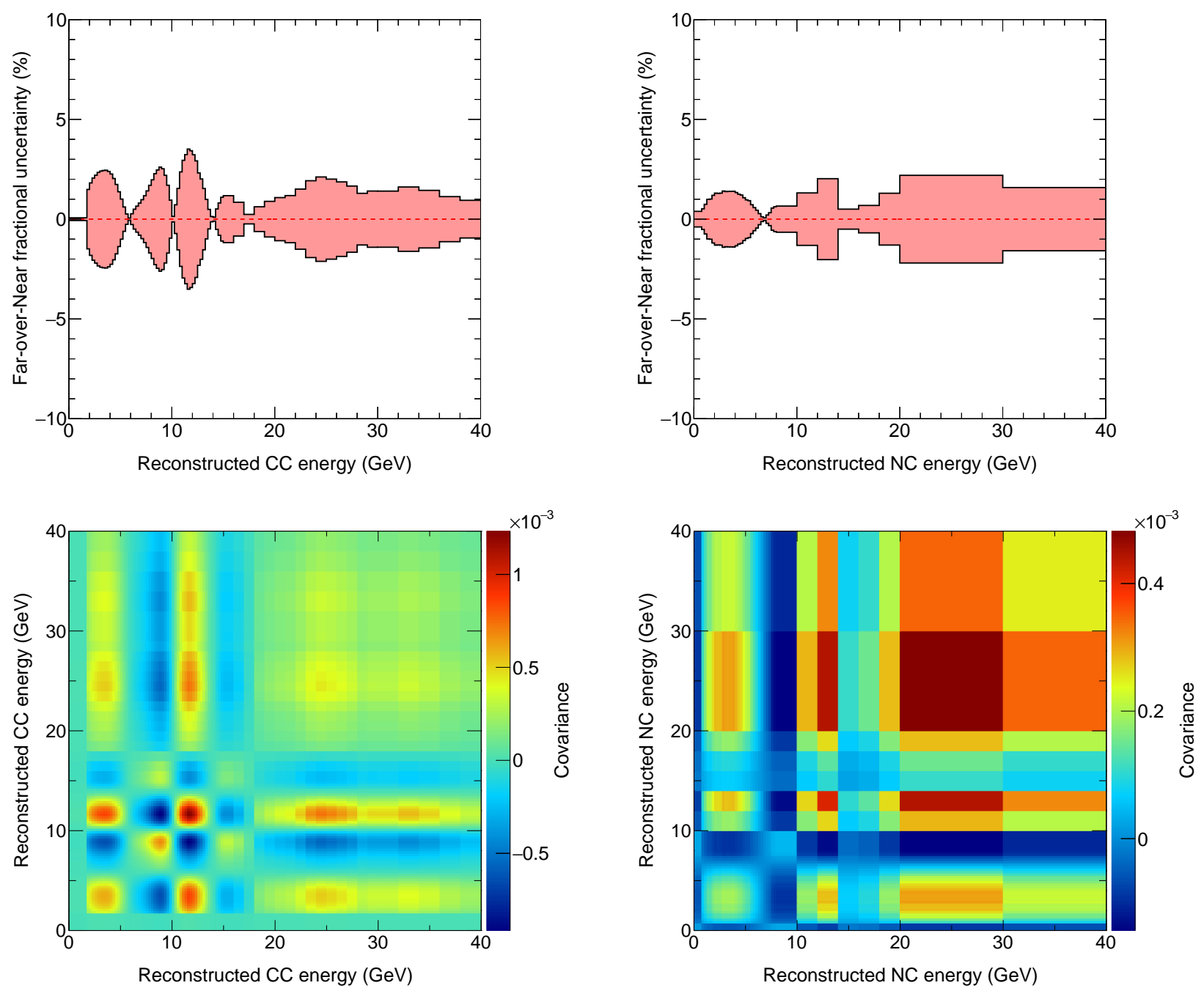

Figure 5.22: The flux modification systematic uncertainty for the MINOS/MINOS+ samples. The fractional uncertainty in the CC (top left) and $\mathrm{NC}$ (top right) Far-over-Near ratios and the corresponding covariance matrices for the $\mathrm{CC}$ (bottom left) and $\mathrm{NC}$ (bottom right) samples. 

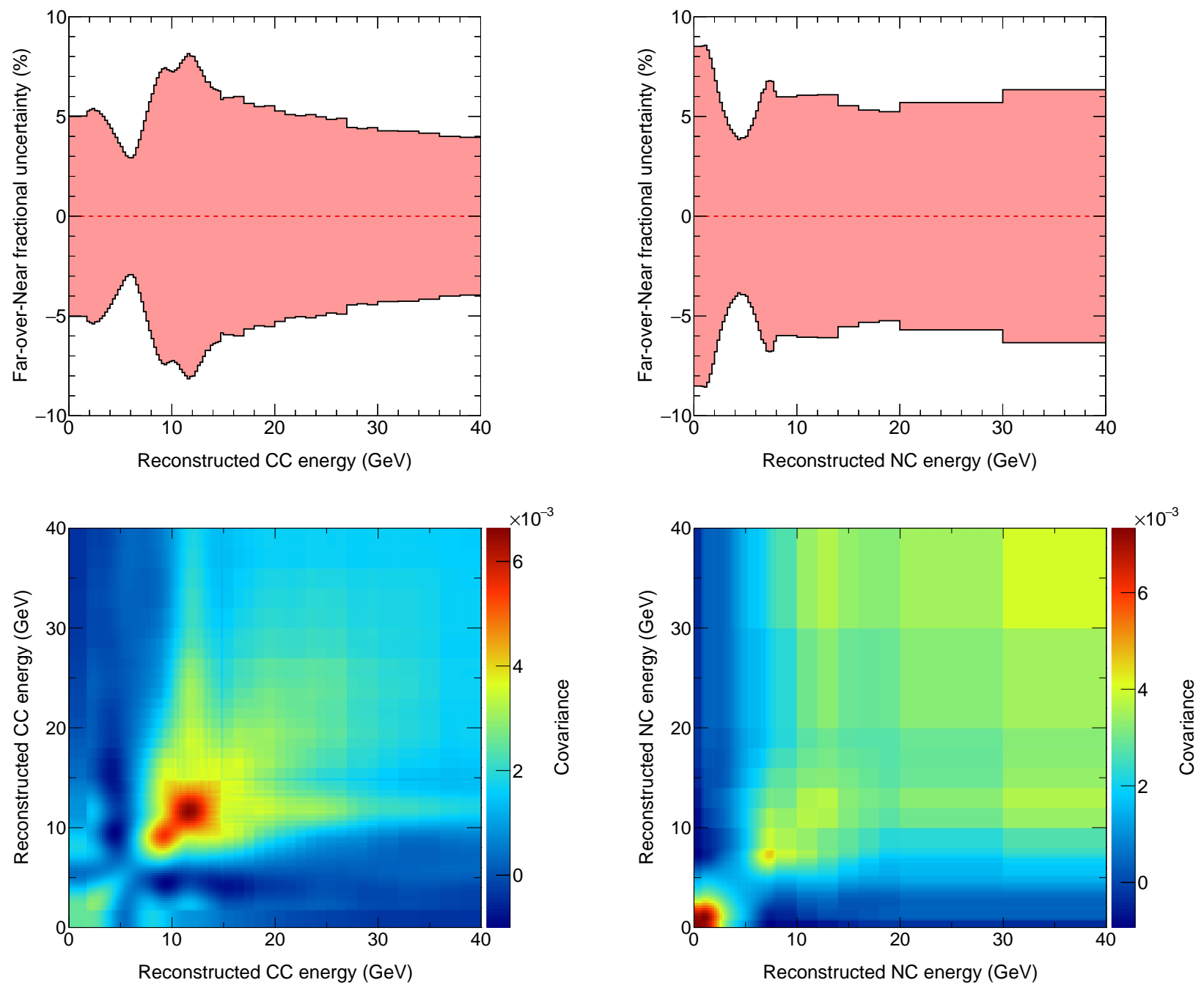

Figure 5.23: The total systematic uncertainty for the MINOS/MINOS+ samples. The fractional uncertainty in the CC (top left) and $\mathrm{NC}$ (top right) Far-over-Near ratios and the corresponding covariance matrices for the $\mathrm{CC}$ (bottom left) and NC (bottom right) samples. 

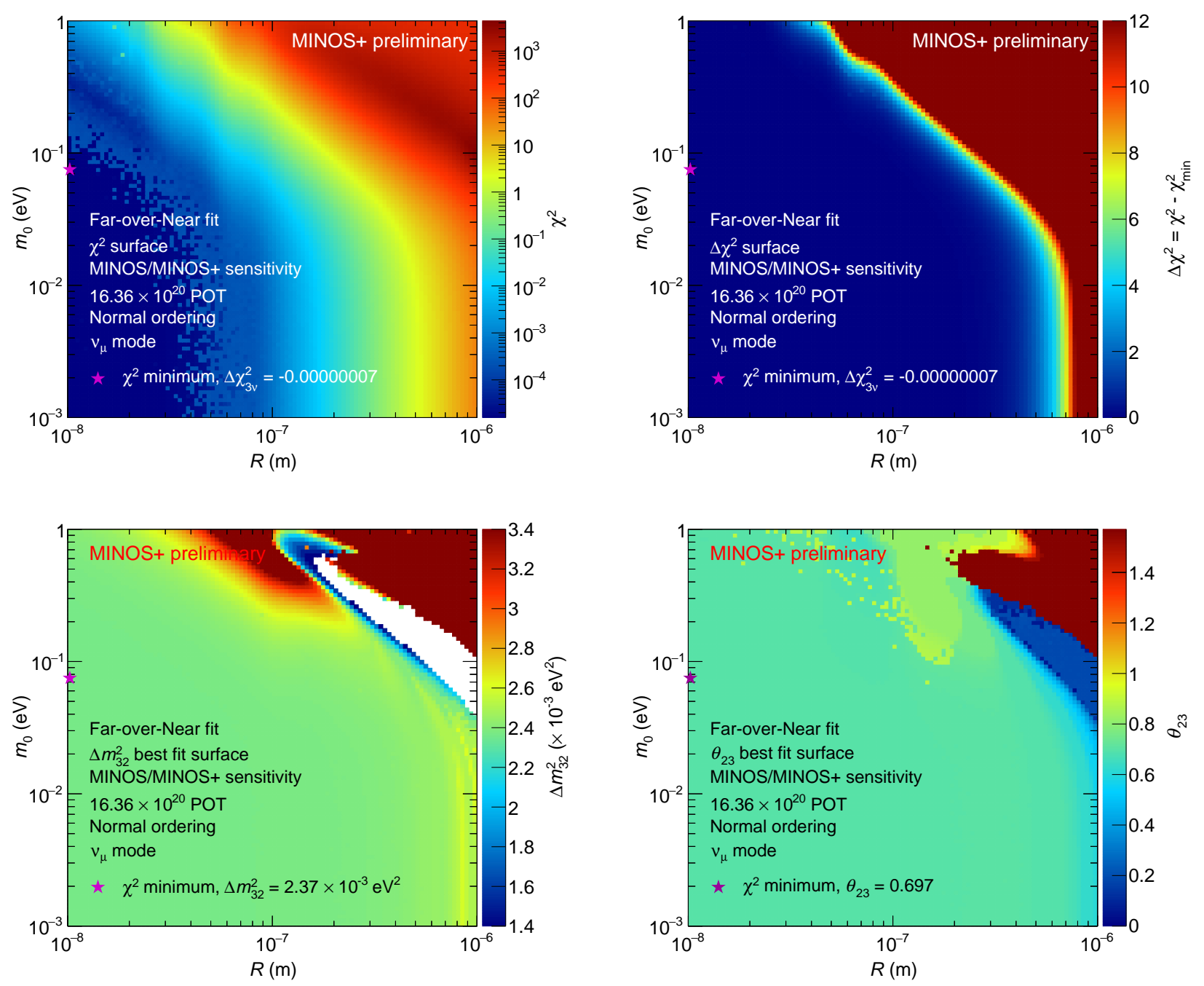

Figure 5.24: The sensitivity surfaces for the LED model in the $\left(R, m_{0}\right)$ plane for a simulated MINOS/MINOS+ exposure of $16.36 \times 10^{20}$ POT and assuming three-flavor simulated data: the $\chi^{2}$ surface (top left), the $\Delta \chi^{2}$ surface (top right), and the best fit values for $\Delta m_{32}^{2}$ (bottom left) and $\theta_{23}$ (bottom right). 

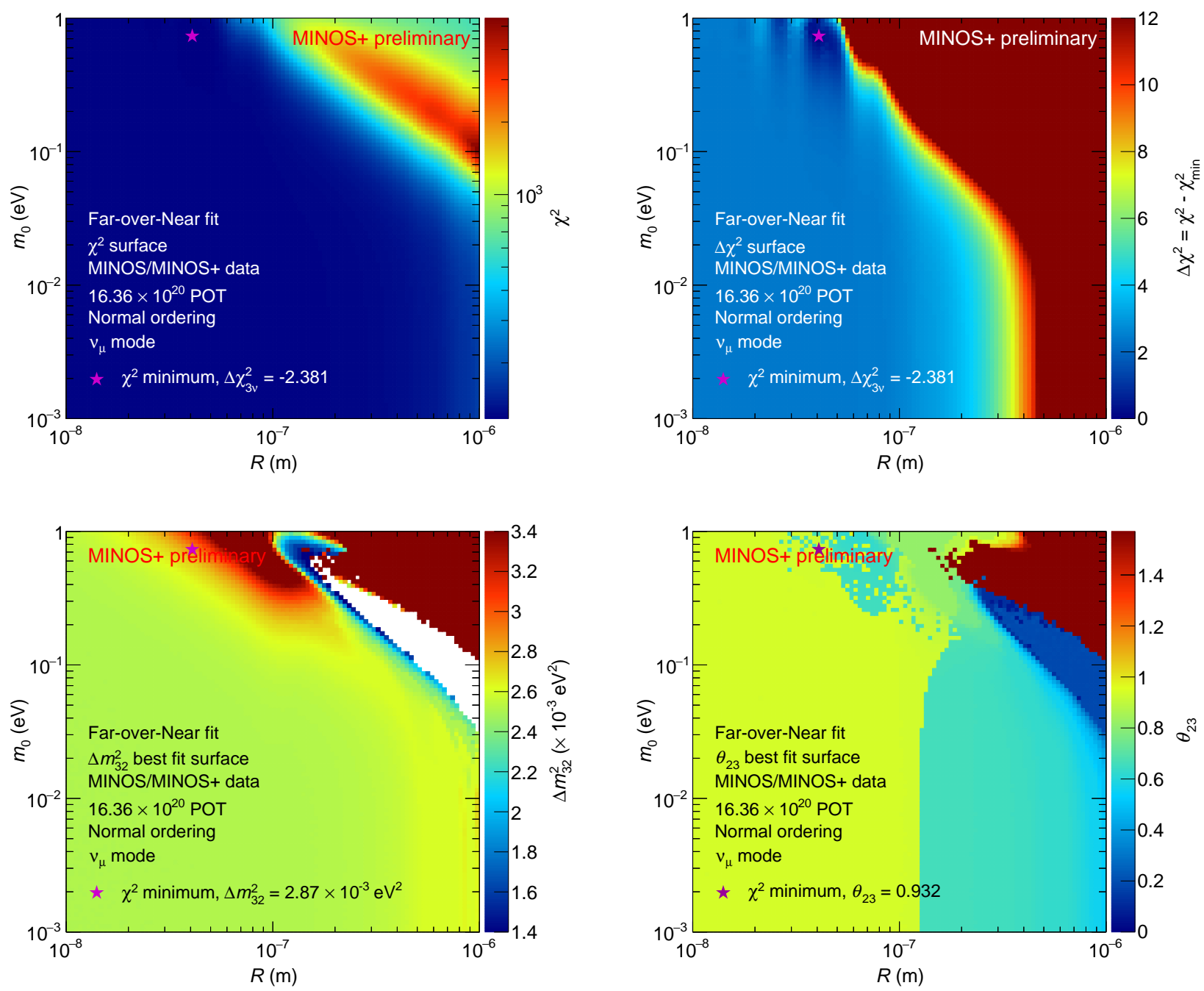

Figure 5.25: The data surfaces for the LED model in the $\left(R, m_{0}\right)$ plane for the MINOS/MINOS+ exposure of $16.36 \times 10^{20}$ POT: the $\chi^{2}$ surface (top left), the $\Delta \chi^{2}$ surface (top right), and the best fit values for $\Delta m_{32}^{2}$ (bottom left) and $\theta_{23}$ (bottom right). 


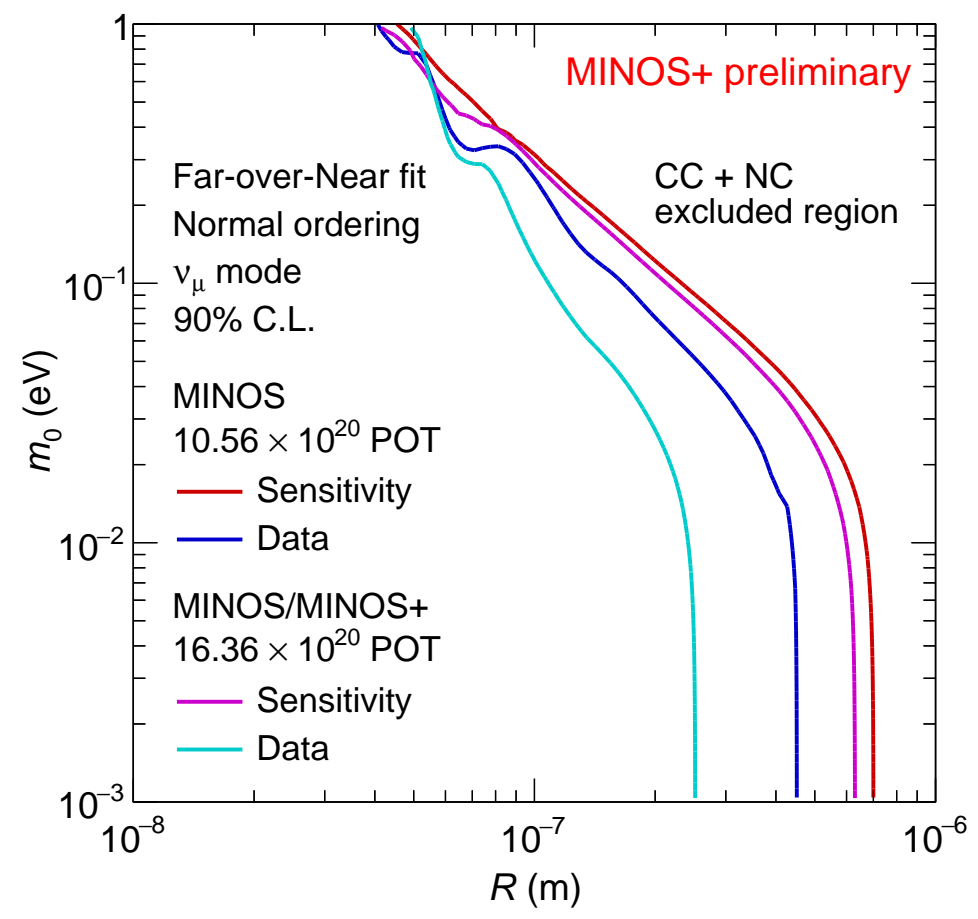

Figure 5.26: Comparison of the Far-over-Near global 90\% C.L Asimov sensitivity and data coverages for the MINOS samples only and for the MINOS/MINOS+ samples. 


\section{Chapter 6}

\section{A Two-Detector Analysis}

This chapter presents a two-detector analysis of the LED model based on the full MINOS accelerator beam neutrino dataset used in the Far-over-Near analysis, and adding the first two years of MINOS+ accelerator beam neutrino data with an exposure of $5.80 \times 10^{20}$ POT, for the NuMI beam operated in the medium-energy $v_{\mu}$ mode and with a neutrino peak energy of $7 \mathrm{GeV}$. The two-detector analysis simultaneously exploits both MINOS detectors to improve the sensitivity to large extra dimensions in the LED model. The addition of MINOS+ data significantly increases the neutrino event counts at higher energies, away from the three-flavor minimum, where the LED model effects are larger. To improve the NuMI neutrino flux simulation, the PPFX hadron production weights developed by MINERvA are employed to correct the FLUGG neutrino flux prediction.

Section 6.1 discusses the two-detector analysis strategy and is followed by a discussion of the systematic uncertainties in Section 6.2. The sensitivity and data $\chi^{2}$ surfaces and the Feldman-Cousins limit are shown in the final two sections. 


\subsection{Analysis Method}

As discussed in Chapter 5, the Far-over-Near ratio explicitly reduces systematic uncertainties that affect both detectors. However, this method also comes with disadvantages. Formula (5.7) demonstrates that the statistical uncertainty on the Far-over-Near ratio is dominated by the statistical uncertainty of the FD. As such, the statistical power of the ND is suppressed and not fully exploited. This reduces the sensitivity of the LED analysis at larger $m_{0}$ values, for which oscillations can occur along the ND baseline. In addition, the Far-over-Near analysis is not sensitive to normalization shifts in the neutrino energy spectra in both detectors, as this effect is not visible through the ratio of the neutrino energy spectra. A simultaneous fit to the ND and FD CC and $\mathrm{NC}$ samples is employed in the two-detector analysis, resolving the two disadvantages mentioned above.

\subsubsection{Correcting the FLUGG Neutrino Flux Prediction}

A correction to the FLUGG neutrino flux prediction is derived to improve the neutrino flux simulation [140]. It is known from Ref. [141] that the $\pi^{+}$to $\mathrm{K}^{+}$production ratio is well described by FLUKA. The hadron production weights in the FLUKA simulation are optimized based on a simultaneous fit to

- the simulated neutrino flux from $\pi^{+}$neutrino parents corrected using the PPFX prediction, in the $0-20 \mathrm{GeV}$ range, and 
- an ND data sample in the 40-60 GeV range,

where both the simulated neutrino flux and the ND data correspond to NuMI operating conditions with the magnetic horns disabled such that there are no beam focusing effects involved. The fit to the PPFX-weighted MC sample from $\pi^{+}$parents ensures a good parametrization of the $\pi^{+}$neutrino parent distribution below $20 \mathrm{GeV}$, which only depends on hadron production experiment data and not on NuMI data, and together with the FLUKA $\pi^{+}$to $\mathrm{K}^{+}$ production ratio is used to predict the $\mathrm{K}^{+}$neutrino parent distribution. The fit to ND data ensures a good parametrization of the $\mathrm{K}^{+}$neutrino parent distribution. The fit is done between 40 and $60 \mathrm{GeV}$, outside of the reconstructed neutrino energy range used in the two-detector analysis. The resulting hadron production parametrization weights are used to generate a correction weight for the nominal FLUGG neutrino flux prediction. The uncertainties on these weights provide a robust estimate of the systematic uncertainty in the hadron production, shown in Section 6.2.8. This process was done separately for the MINOS and MINOS+ samples. Figure 6.1 shows the fitting procedure and the resulting effect on the reconstructed neutrino energy spectrum at the ND for the MINOS+ sample.

\subsubsection{Test Statistic and Ghost Fitter}

Similar to the Far-over-Near analysis, a $\chi^{2}$ test statistic is used to simultaneously compare the LED predictions for the $\mathrm{CC}$ and $\mathrm{NC}$ samples in 

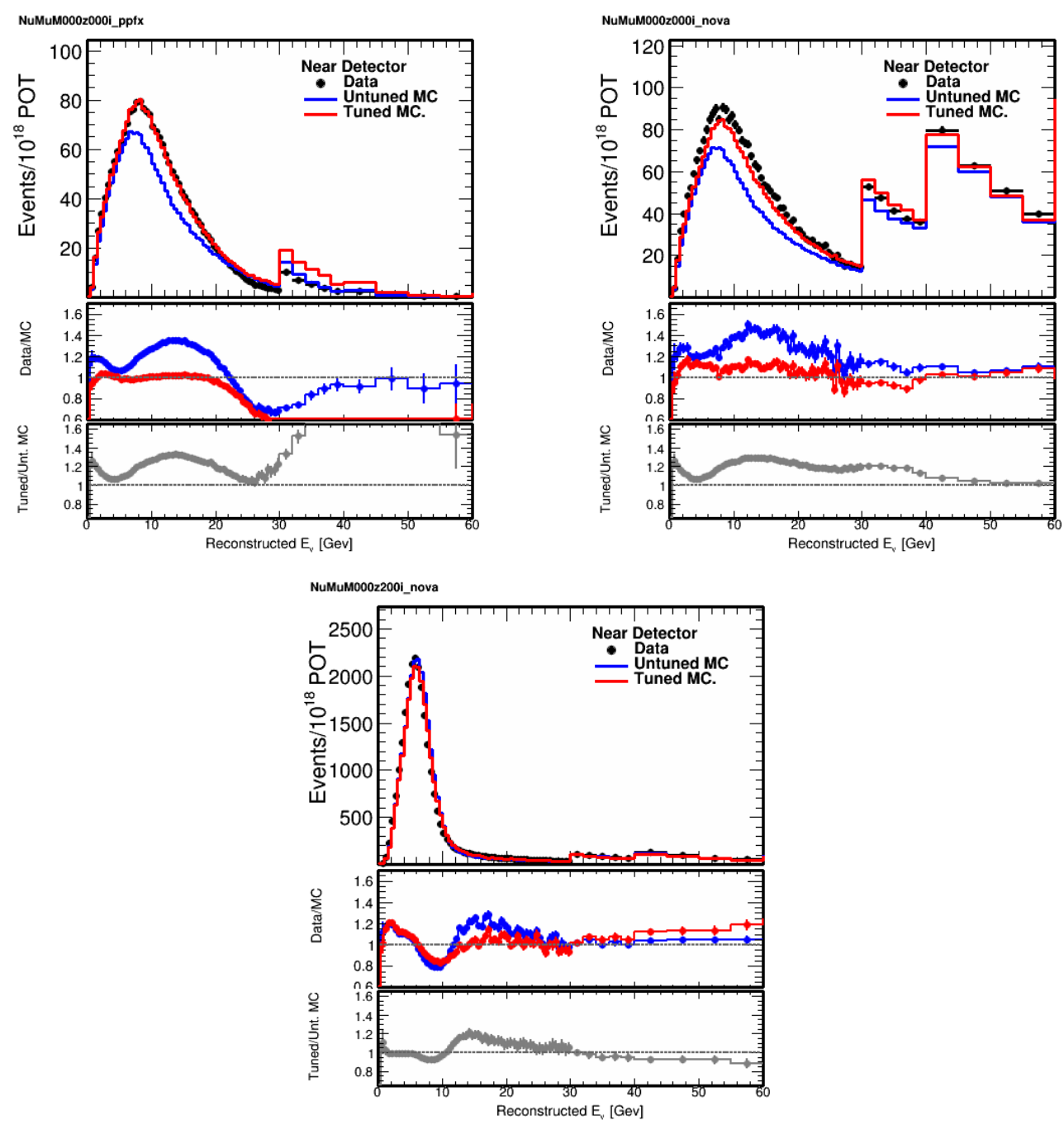

Figure 6.1: Correcting the FLUGG neutrino flux prediction. (Top row) Optimizing the FLUKA hadron production weights by performing a simultaneous fit to the simulated neutrino flux from $\pi^{+}$neutrino parents corrected using the PPFX prediction in the $0-20 \mathrm{GeV}$ range (left) and a MINOS+ ND data sample in the $40-60 \mathrm{GeV}$ range (right), with the magnetic horns disabled in both cases to remove any effects related to beam focusing. (Bottom) The reconstructed neutrino neutrino energy spectrum at the ND for MINOS+ ND data with the magnetic horns turned on (black points) and MC simulation before (blue line) and after (red line) applying the hadron production weights obtained from the fits shown in the top row panels. These figures are taken from Ref. [140]. 
the ND and FD to the observed energy spectra

$$
\chi^{2}=\chi_{\mathrm{CC}}^{2}+\chi_{\mathrm{NC}}^{2}
$$

with

$$
\chi_{\mathrm{CC} / \mathrm{NC}}^{2}=\sum_{i, j=1}^{N}\left(o_{i}-p_{i}\right)\left[V^{-1}\right]_{i j}\left(o_{j}-p_{j}\right)
$$

where $o_{i}$ and $p_{i}$ are the observed and predicted number of events in energy bin $i$, respectively, and $V$ is the sum of statistical and systematic covariance matrices including bin-to-bin correlations.

In each sample, the FD and ND energy spectra are glued together, such that elements 1 to $k$ in $O=\left(o_{1}, \ldots, o_{k}, o_{k+1}, o_{N}\right)$ and $P=\left(p_{1}, \ldots, p_{k}, p_{k+1}, p_{N}\right)$ correspond to the bins in the FD energy spectrum, while elements $k+1$ to $N$ correspond to the bins in the ND energy spectrum. Similarly, the covariance matrix $V$ is divided into four quadrants for which the top left quadrant corresponds to the FD covariance matrix, the bottom right quadrant corresponds to the ND covariance matrix, and the cross-term matrices describe the covariance between the detectors. This is illustrated in Fig. 6.2. Unlike in the Far-over-Near analysis where normalization effects can cancel out when taking the ratio of the spectra, the two-detector analysis $\chi^{2}$ test statistic does not require an ND neutrino flux penalty term because both the ND and FD spectra are fitted simultaneously.

Two new libraries of MINOS and MINOS+ LED predictions were made at the start of the analysis. The binning schemes for the energy spectra of the 4 


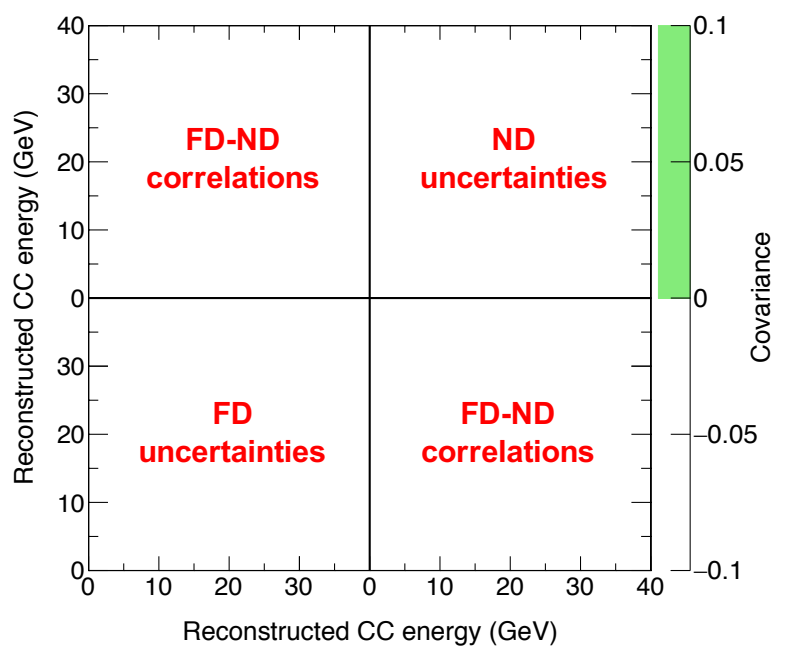

Figure 6.2: The structure of the two-detector covariance matrix for the CC sample. It should be noted that the $(1,1)$ bin of the matrix corresponds to the bottom left corner in this figure. A similar matrix structure is used for the NC sample.

samples are illustrated in Fig. 6.3, where the effect of multilinear interpolation is shown for two points in the LED parameter space. As is the case in the Farover-Near analysis, the effects depend on the value of the oscillation parameters and do not exceed a few percent.

The values and constraints on the neutrino oscillation parameters used in the two-detector analysis to minimize Formula (6.1) are the same as those listed in Table 5.1 for the Far-over-Near analysis.

\subsection{Systematic Uncertainties}

The sources of systematic uncertainties presented in Section 5.2 in the context of the Far-over-Near analysis are reevaluated for the two-detector anal- 

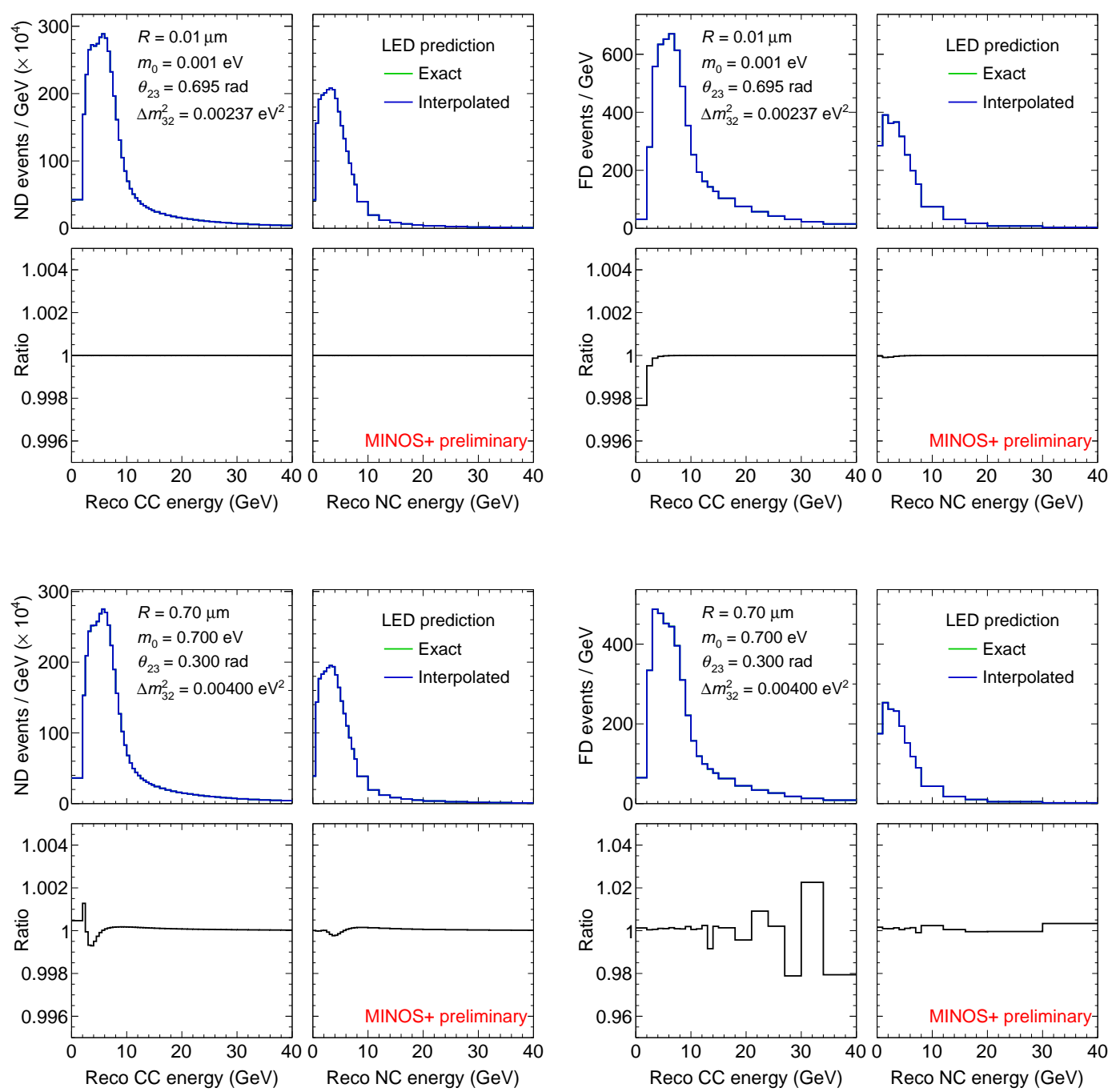

Figure 6.3: Comparison of the ND (left) and FD (right) LED two-detector predictions at two points in the LED parameter space obtained through exact calculation (green line) or through multilinear interpolation between predictions calculated for neighboring LED grid points (blue line). 
ysis, with special emphasis on the magnitudes of the bin-to-bin correlations in the two-detector energy spectra. This is discussed in more detail in Ref. [142].

The reconsidered total and individual systematic uncertainties are shown in Fig. 6.4 through 6.13 and briefly discussed below.

Similar to Formula (5.17), uncertainty bands are constructed using

$$
\frac{\mathrm{MC}_{\text {shifted }}}{\mathrm{MC}_{\text {nominal }}}-1
$$

where MC represents the simulated two-detector spectrum. The covariance matrices are constructed from the uncertainty bands according to the procedure outlined in Section 5.2.2. It should be noted that the systematic covariance matrices of an individual systematic uncertainty source for the combined MINOS/MINOS+ samples are obtained from combining the shifted MINOS and MINOS+ energy spectra before generating the uncertainty band with Formula (6.3). Combining the shifted spectra before generating the covariance matrices leads to smaller uncertainties then combining the covariance matrices of the MINOS and MINOS+ samples because the uncertainties affect different parts of the energy spectrum for MINOS and MINOS+.

The covariance between energy bins within each detector and the covariance between energy bins of both detectors in the two-detector covariance matrix allow the cancellation of systematic uncertainties for highly correlated bins in a similar fashion to the explicit cancellation of systematic uncertainties in the Far-over-Near method. The interplay between both detectors through 
the two-detector approach thus provides a cross-check to distinguish possible LED signals from systematic fluctuations.

\subsubsection{Total Systematic Uncertainty}

The total systematic uncertainty in the two-detector samples and the corresponding covariance matrices for the $\mathrm{CC}$ and $\mathrm{NC}$ samples are shown in Fig. 6.4. The individual systematic covariance matrices discussed in the following sections are added through matrix addition, thereby treating them as independent uncertainties. The shape of the energy scale and hadron production uncertainty bands dominate the overall shape of the total systematic uncertainty band, while uncertainties from sources such as the cross sections and background increase its overall magnitude.

\subsubsection{Acceptance Uncertainty}

The acceptance uncertainty in the two-detector samples and the corresponding covariance matrices for the $\mathrm{CC}$ and $\mathrm{NC}$ samples are shown in Fig. 6.5. The acceptance systematic uncertainty is considered to be uncorrelated binto-bin.

\subsubsection{Normalization Uncertainty}

The normalization uncertainty in the two-detector samples and the corresponding covariance matrices for the $\mathrm{CC}$ and NC samples are shown in Fig. 6.6. The normalization uncertainty sources from the Far-over-Near analy- 

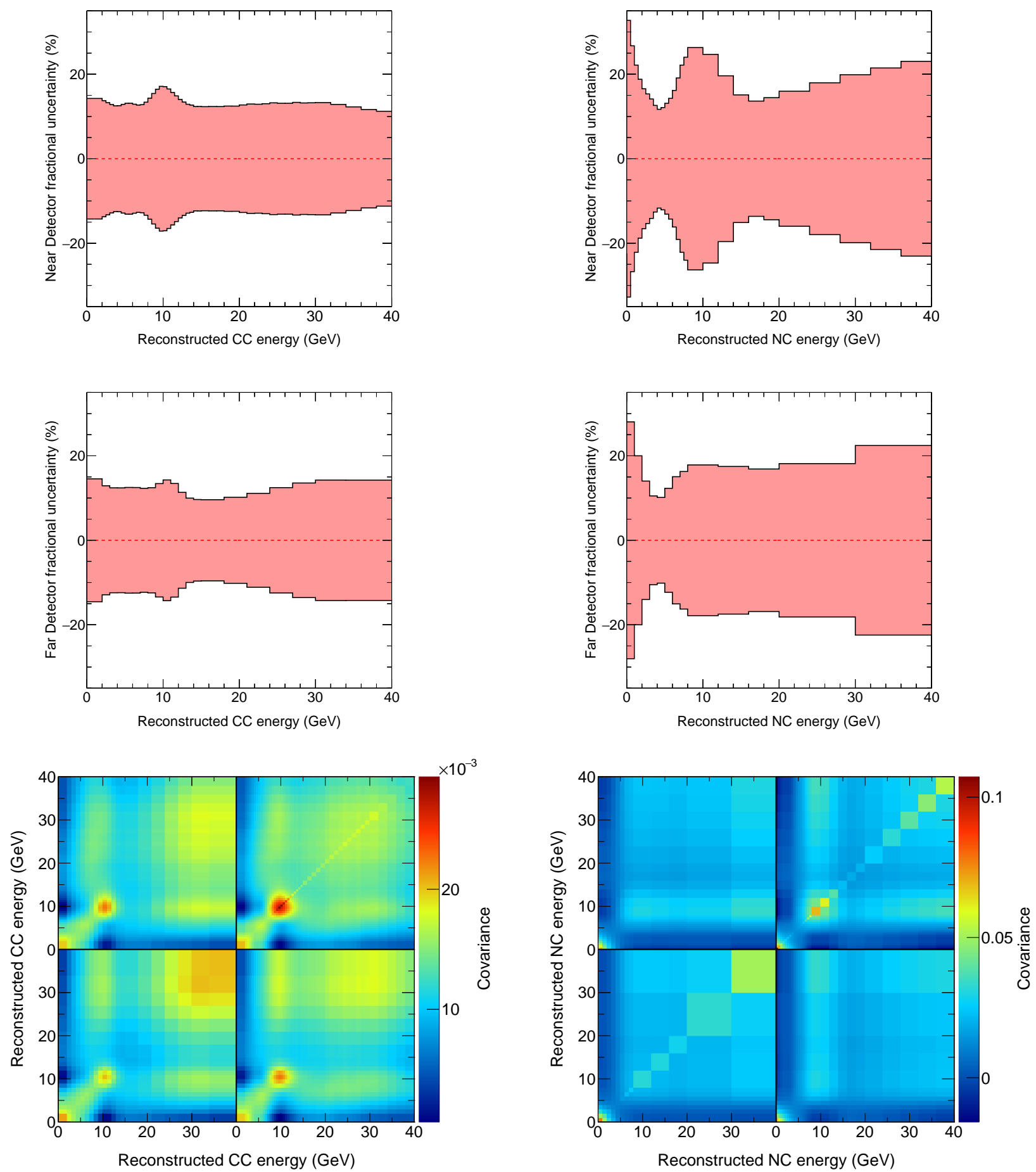

Figure 6.4: The total systematic uncertainty for the MINOS/MINOS+ samples. The fractional uncertainty in the ND CC and NC (top left and right) and FD $\mathrm{CC}$ and $\mathrm{NC}$ (middle left and right) samples and the corresponding two-detector covariance matrices for the CC (bottom left) and NC (bottom right) samples. 

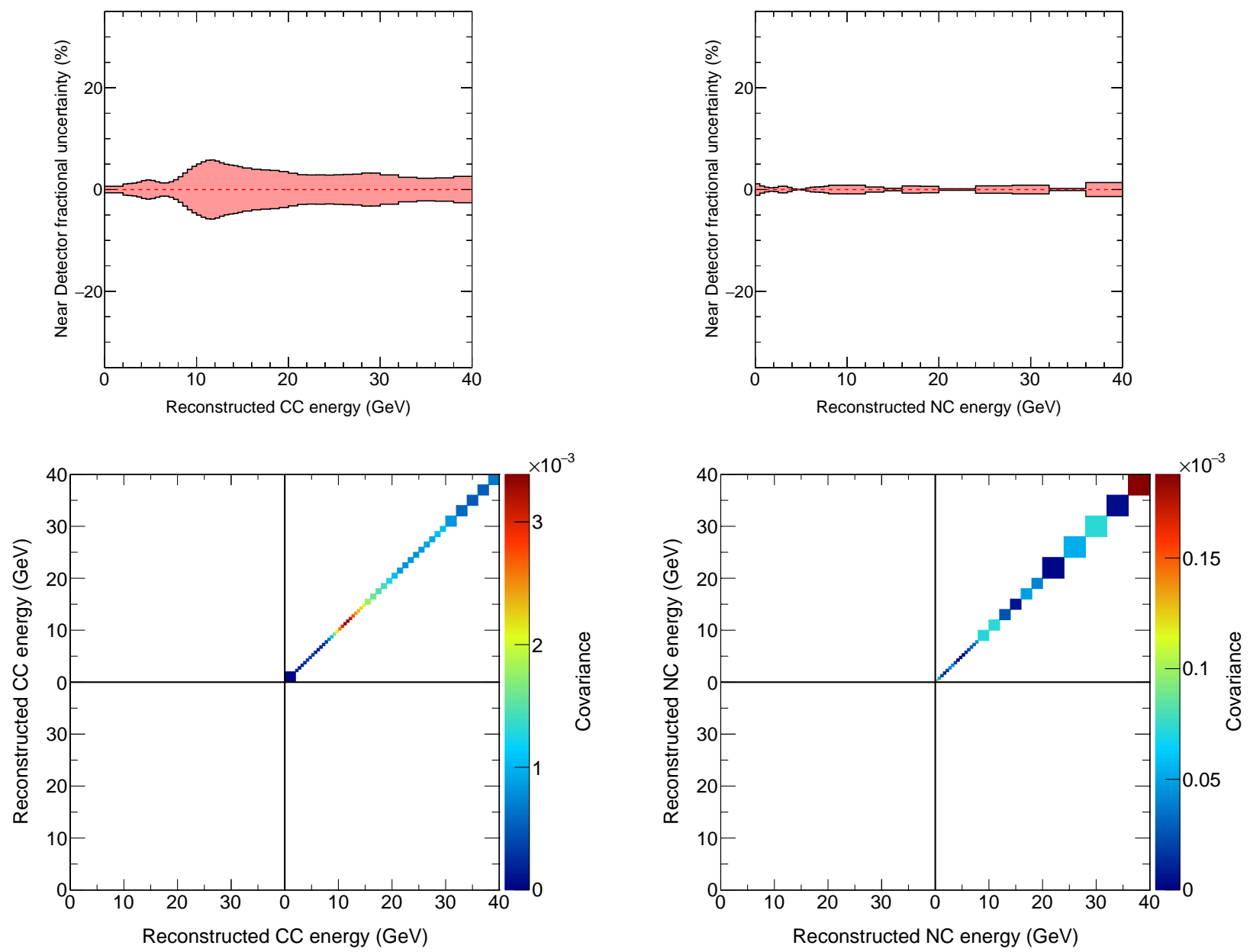

Figure 6.5: The acceptance systematic uncertainty for the MINOS/MINOS+ samples. The fractional uncertainty in the ND CC and NC (top left and right) samples and the corresponding two-detector covariance matrices for the $\mathrm{CC}$ (bottom left) and NC (bottom right) samples. 
sis are reconsidered and either treated as fully correlated or fully uncorrelated. For example, steel or scintillator thickness uncertainties, as well as uncertainty in FD detector live time all effectively change the exposure in the detectors by changing the detector mass or the POT exposure, respectively. As such, these uncertainty sources change the entire energy spectra in the same way and are treated as fully correlated between energy bins. However, the largest normalization uncertainty sources, including selection and fiducial biases mentioned in Section 5.2.5 are treated as uncorrelated and the resulting covariance matrix is mostly uncorrelated, as apparent from the large difference in magnitude between the diagonal and off-diagonal elements of the covariance matrices in the bottom panels of Fig. 6.6. Hence, the normalization systematic uncertainty provides only small uncertainty cancellation effects in the two-detector framework.

\subsubsection{Neutral Current Uncertainty}

The left panels of Fig. 6.7 show the NC selection uncertainty bands in the ND and FD. The NC selection requirements discussed in Section 4.1 are independent for both detectors, such that the effects are fully correlated within each detector and uncorrelated between both detectors. This is illustrated in the right panel of Fig. 6.7. 

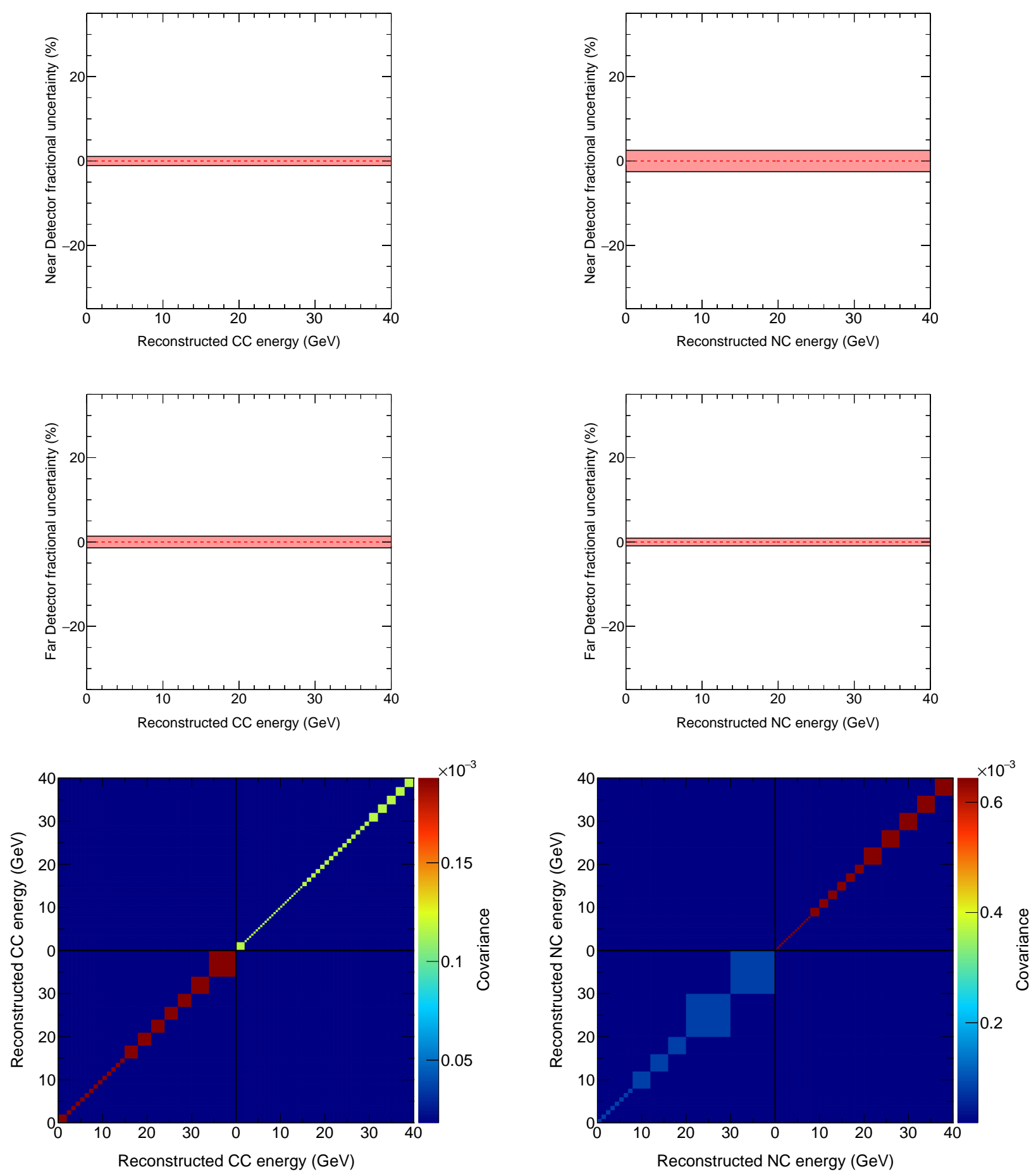

Figure 6.6: The normalization systematic uncertainty for the MINOS/MINOS+ samples. The fractional uncertainty in the ND CC and NC (top left and right) and FD CC and NC (middle left and right) samples and the corresponding two-detector covariance matrices for the CC (bottom left) and NC (bottom right) samples. 

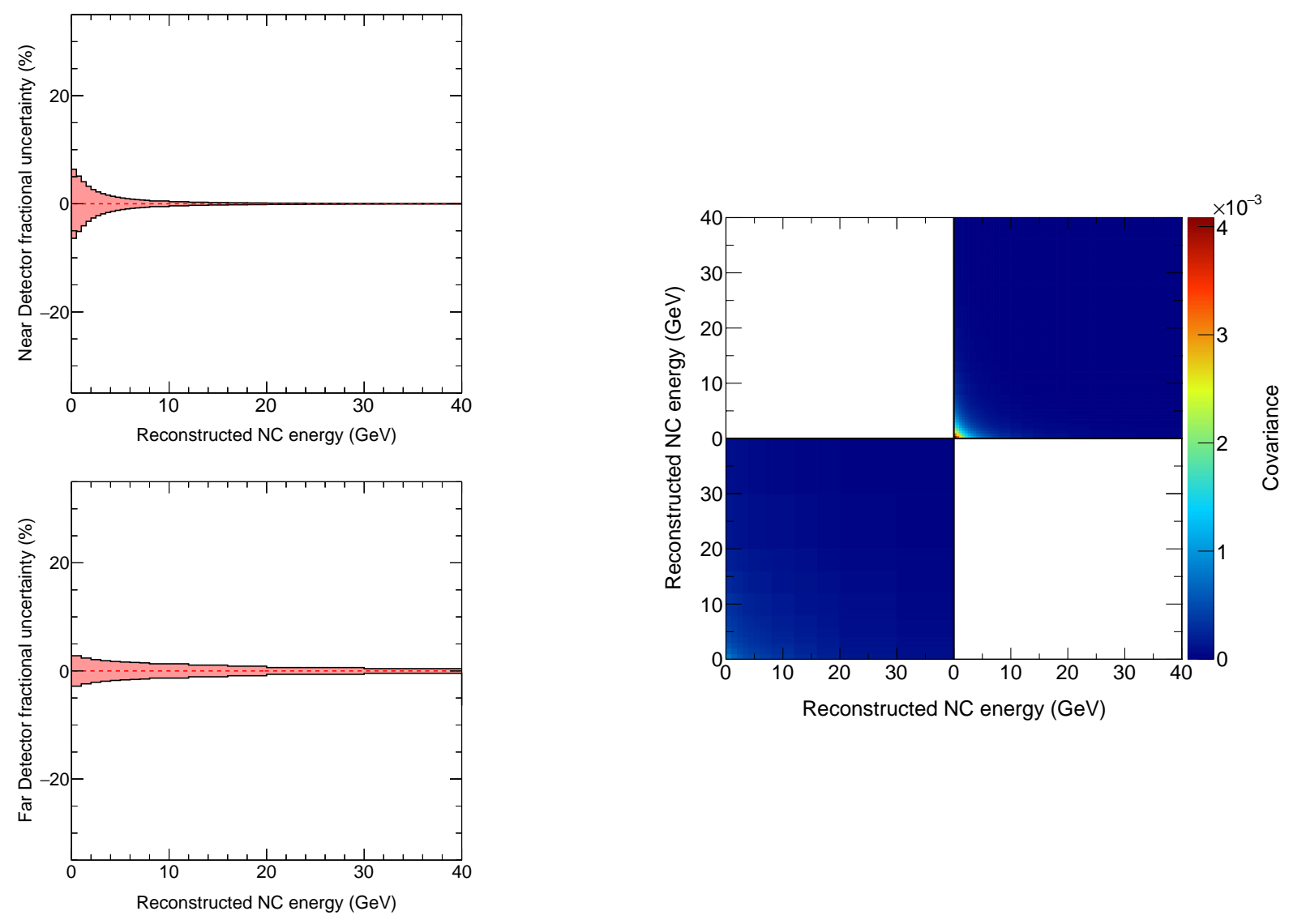

Figure 6.7: The NC selection systematic uncertainty for the MINOS/MINOS+ samples. The fractional uncertainty in the ND NC (top left) and FD NC (bottom left) samples and the corresponding two-detector covariance matrix for the NC (right) samples. 


\subsubsection{Energy Scale Uncertainties}

By separating the track range and track curvature systematic uncertainties, mentioned in Section 5.2.7, they can individually be treated as fully correlated uncertainties. Similarly, the two components in Formula (5.21) are separated, with the absolute energy scale uncertainty from the calibration process treated as fully correlated and the energy dependent term treated as uncorrelated. Figure 6.8 shows the energy scale uncertainties in the two-detector samples and the corresponding covariance matrices for the $\mathrm{CC}$ and $\mathrm{NC}$ samples. They are the one of the largest contributions to the overall systematic uncertainty, but due to significant correlations between the energy bins within each detector and between both detectors, the effect on the overall sensitivity is largely reduced, as will be shown in Section 6.4.1.

\subsubsection{Neutrino Cross Section Uncertainty}

Figure 6.9 shows the cross section uncertainty in the two-detector samples and the corresponding covariance matrices for the $\mathrm{CC}$ and $\mathrm{NC}$ samples. Cross sections are well defined and changing a parameter causes a shift in the entire neutrino energy spectrum, such that the neutrino cross section uncertainties are fully correlated. Because the MINOS detectors are functionally identical, any difference in systematic uncertainty between the detectors is a consequence of the difference in the energy spectra. 

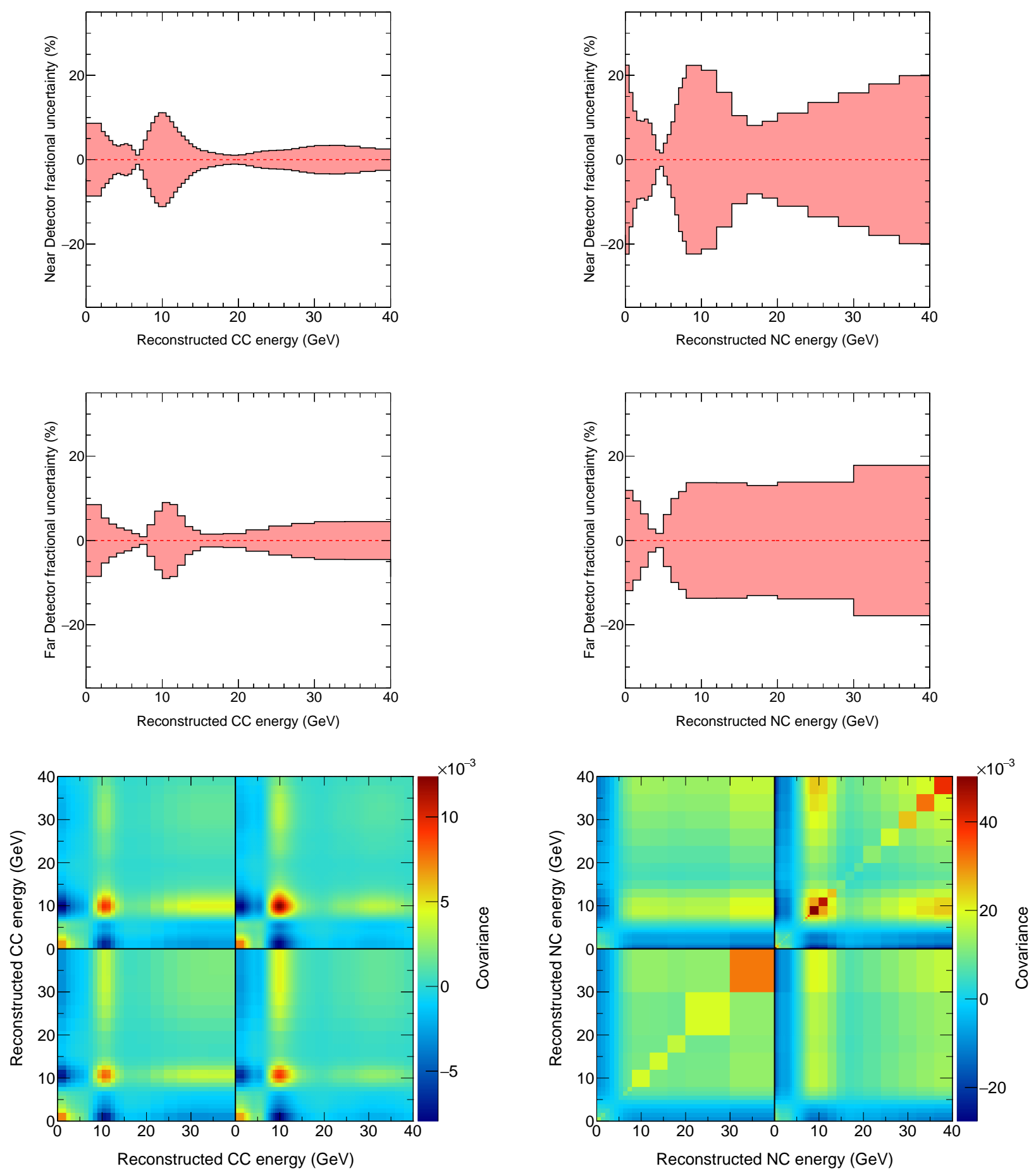

Figure 6.8: The energy scale systematic uncertainties for the MINOS/MINOS+ samples. The fractional uncertainty in the ND CC and NC (top left and right) and FD CC and NC (middle left and right) samples and the corresponding two-detector covariance matrices for the CC (bottom left) and NC (bottom right) samples. 

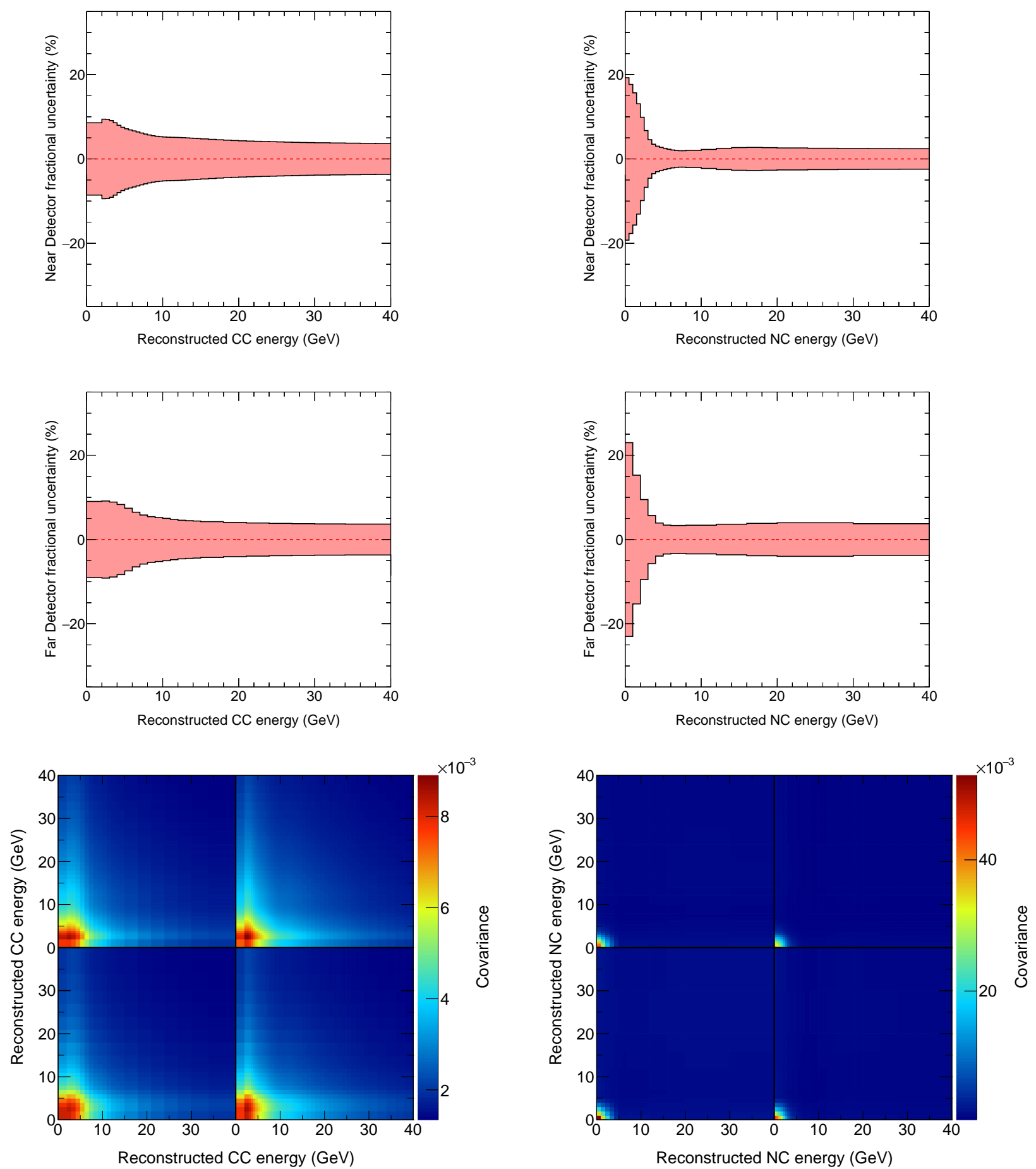

Figure 6.9: The neutrino cross section systematic uncertainty for the MINOS/MINOS+ samples. The fractional uncertainty in the ND CC and NC (top left and right) and FD CC and NC (middle left and right) samples and the corresponding two-detector covariance matrices for the CC (bottom left) and NC (bottom right) samples. 


\subsubsection{Background Uncertainty}

A 30\% systematic uncertainty in the NC background in the CC sample was obtained from a study in Ref. [45] for the first year of MINOS+ operation. This uncertainty was carried over for this analysis. The uncertainty in the CC background in the NC sample is considered correlated between energy bins. Figure 6.10 shows the background uncertainty in the two-detector samples and the corresponding covariance matrices for the $\mathrm{CC}$ and $\mathrm{NC}$ samples.

\subsubsection{Hadron Production Uncertainty}

The uncertainties in the hadron production weights obtained from the simultaneous fit to PPFX-corrected neutrino flux predictions and MINOS or MINOS+ ND data are used to construct the hadron production systematic uncertainties shown in Fig. 6.11.

\subsubsection{Run Period Weight Uncertainty}

In order to employ the PPFX hadron production weights provided by MINERvA to correct the FLUGG neutrino fluxes for MINOS and MINOS+, the MINERvA and MINOS or MINOS+ flux simulations need to be compared. During the MINOS+ era, both MINERvA and MINOS+ only have one neutrino flux simulation and a one-to-one comparison is performed. During the MINOS era, multiple neutrino flux simulations exist for both MINOS and MINERvA due to variations in geometry and composition of the NuMI beam

apparatus. An additional systematic uncertainty is assigned to this match- 

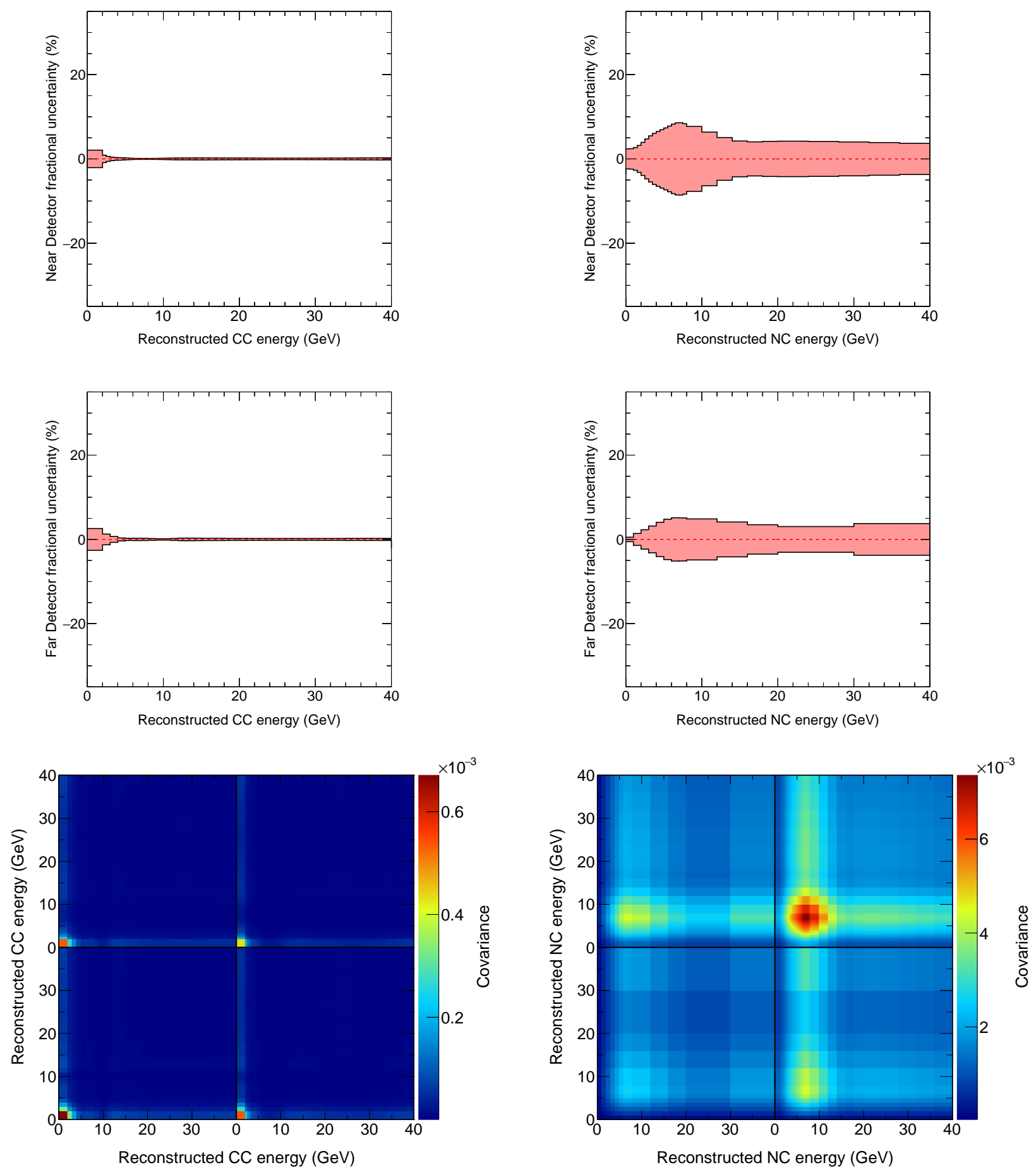

Figure 6.10: The background systematic uncertainty for the MINOS/MINOS+ samples. The fractional uncertainty in the ND CC and NC (top left and right) and FD $\mathrm{CC}$ and $\mathrm{NC}$ (middle left and right) samples and the corresponding two-detector covariance matrices for the CC (bottom left) and NC (bottom right) samples. 

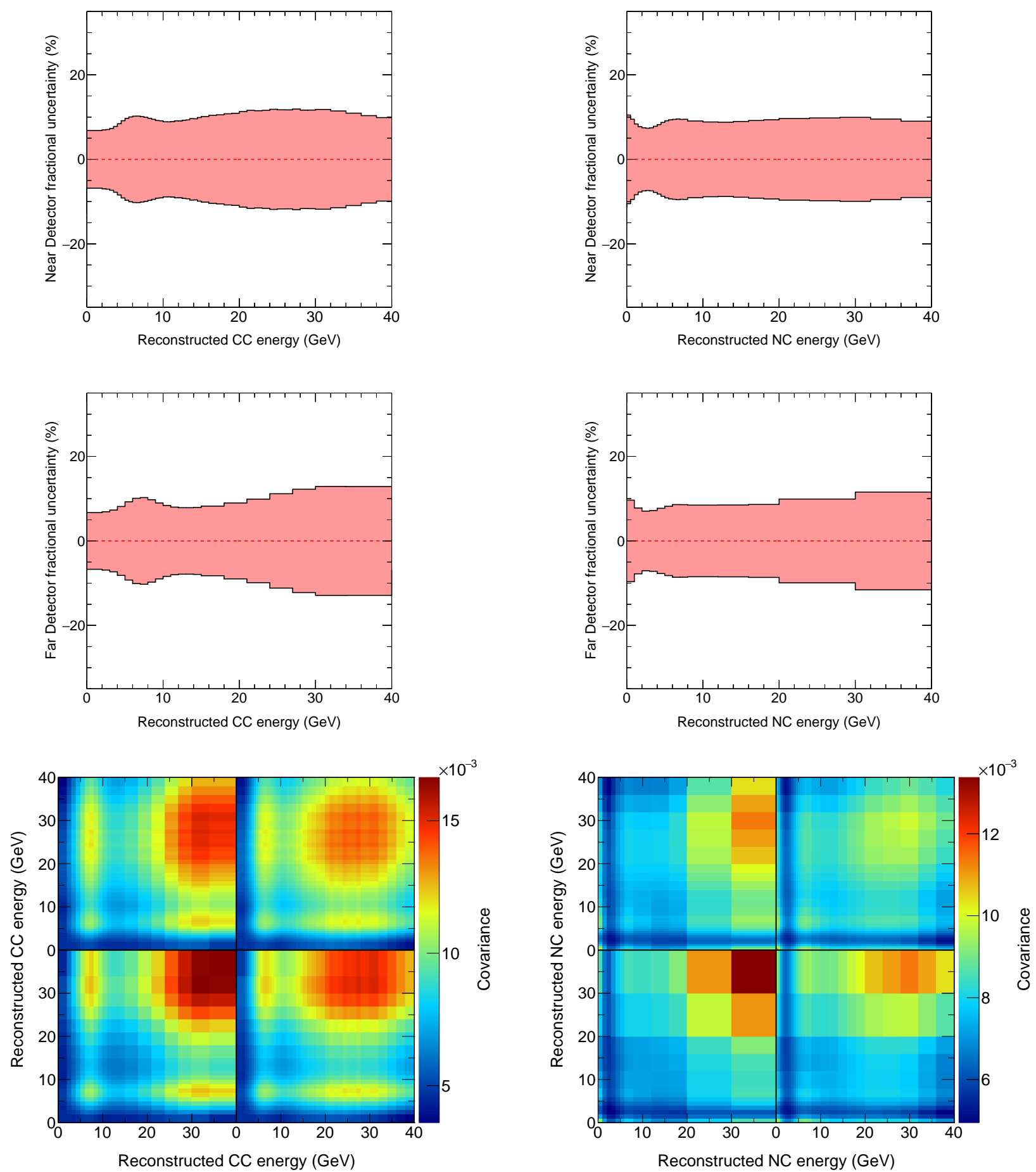

Figure 6.11: The hadron production systematic uncertainty for the MINOS/MINOS+ samples. The fractional uncertainty in the ND CC and NC (top left and right) and FD CC and NC (middle left and right) samples and the corresponding two-detector covariance matrices for the CC (bottom left) and NC (bottom right) samples. 
ing process and is called the run period weight uncertainty [143]. Figure 6.12 shows the run period weight uncertainty in the two-detector samples and the corresponding covariance matrices for the CC and NC samples. These effects

are of the order of $5 \%$ in the MINOS sample but are greatly reduced when combined with the MINOS+ sample.

\subsubsection{Beam Optics Uncertainty}

Figure 6.12 shows the bean optics uncertainty in the two-detector samples and the corresponding covariance matrices for the $\mathrm{CC}$ and $\mathrm{NC}$ samples. 

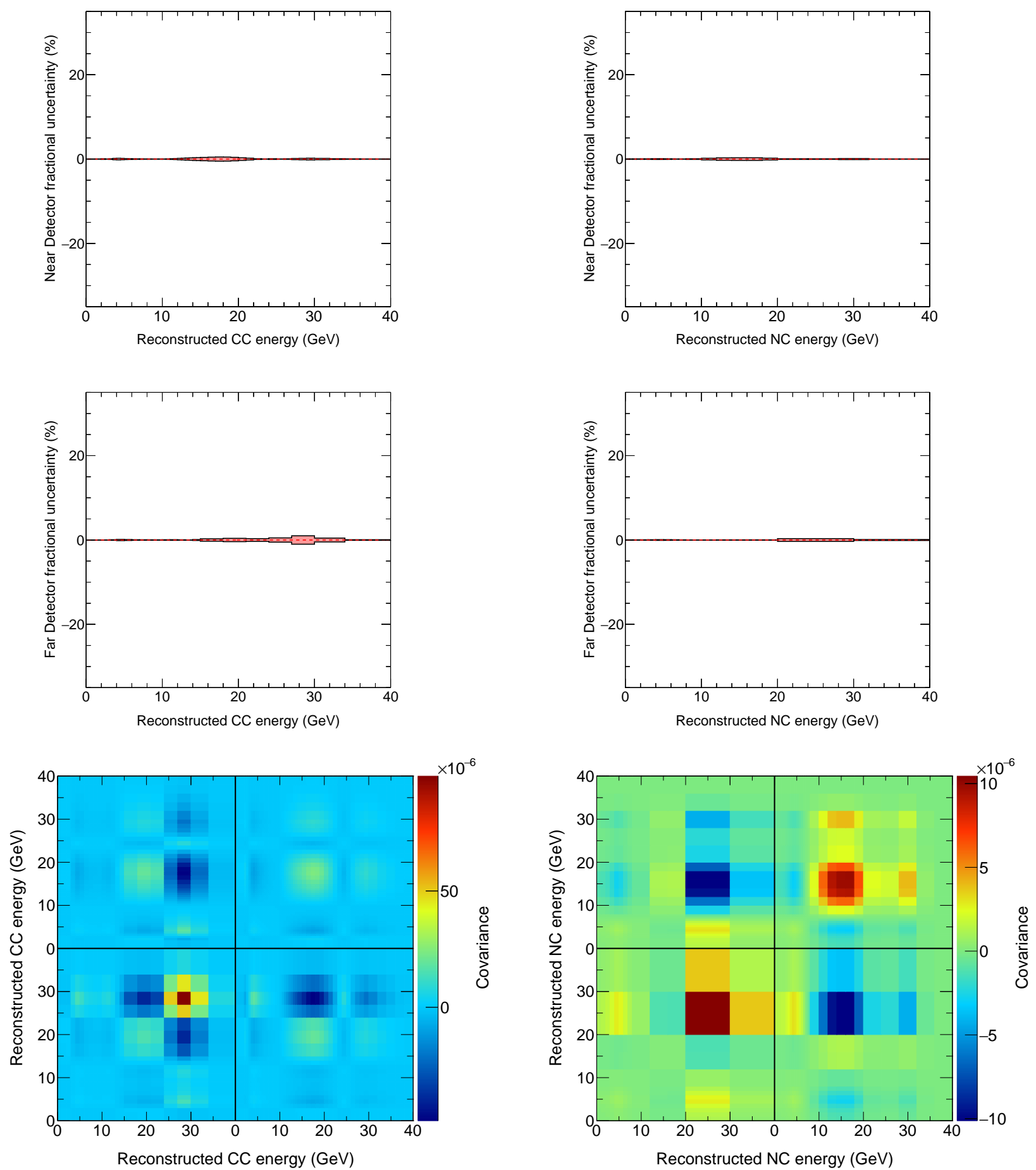

Figure 6.12: The run period weight systematic uncertainty for the MINOS/MINOS+ samples. The fractional uncertainty in the ND CC and NC (top left and right) and FD CC and NC (middle left and right) samples and the corresponding two-detector covariance matrices for the CC (bottom left) and NC (bottom right) samples. 

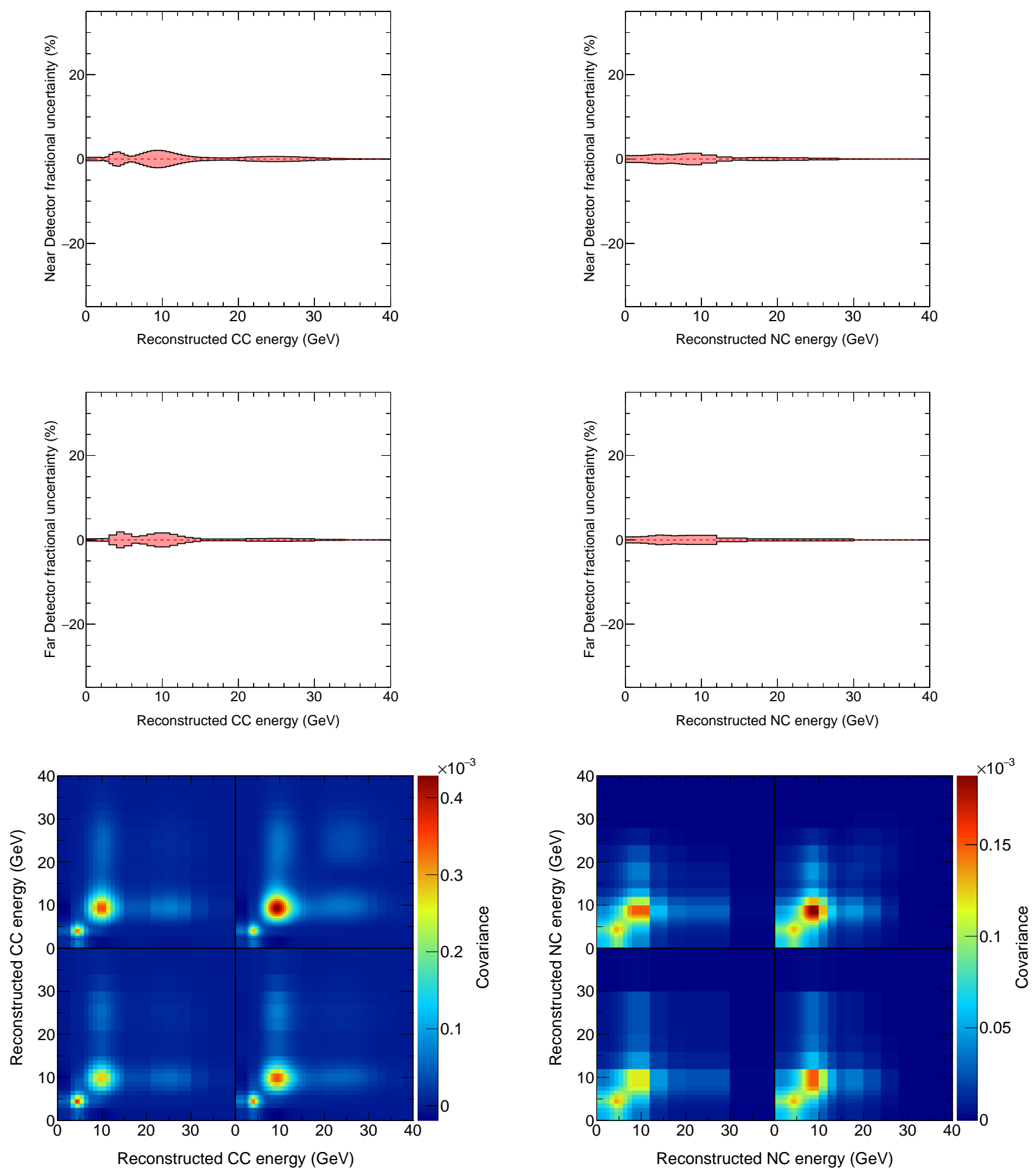

Figure 6.13: The beam optics systematic uncertainty for the MINOS/MINOS+ samples. The fractional uncertainty in the ND CC and NC (top left and right) and FD CC and NC (middle left and right) samples and the corresponding two-detector covariance matrices for the CC (bottom left) and NC (bottom right) samples. 


\subsection{Sensitivity and Data $\chi^{2}$ Surfaces}

The $\chi^{2}$ surfaces for the MINOS/MINOS+ two-detector Asimov sensitivity are shown in Fig. 6.14. The Asimov sensitivity is obtained from comparing the two-detector LED model predictions with simulated three-flavor data, shown in Fig. 4.12, corresponding to the MINOS exposure of $10.56 \times 10^{20}$ POT and the MINOS+ exposure of $5.80 \times 10^{20}$ POT. The values for the three-flavor parameters used to generate the simulated three-flavor data are the same as those used in the Far-over-Near analysis and are listed in Table 5.5. A very flat $\chi^{2}$ surface, with a shallow global minimum, is obtained for most of the $\left(R, m_{0}\right)$ plane where $R \lesssim 10^{-7} \mathrm{~m}$, as is shown in the top left panel of Fig. 6.14. The $\Delta \chi^{2}=\chi^{2}-\chi_{\min }^{2}$ surface is shown in the top right panel, where $\chi_{\min }^{2}$ is the global minimum in the $\chi^{2}$ plane. The bottom panels of Fig. 6.14 show the values of the atmospheric parameters that minimize the $\chi^{2}$ test statistic at a fixed point in the $\left(R, m_{0}\right)$ plane. The white region in the bottom left panel corresponds to $\Delta m_{32}^{2}$ values between $10^{-6}$ and $1.4 \times 10^{-3} \mathrm{eV}^{2}$. The fitting procedure obtains atmospheric parameter values that are consistent with the simulated three-flavor data in the region of the $\left(R, m_{0}\right)$ plane that corresponds to a very flat $\chi^{2}$ surface, whereas significantly different values are obtained in regions where the LED model predicts oscillations inconsistent with the threeflavor paradigm, in particular where oscillations along the ND baseline are predicted.

The same $\chi^{2}$ surfaces are shown in Fig. 6.15 for the MINOS/MINOS+ two-detector data. A shallow global minimum is found at $R=0.010 \mu \mathrm{m}$, 
$m_{0}=0.0018 \mathrm{eV}, \Delta m_{32}^{2}=2.46 \times 10^{-3} \mathrm{eV}^{2}$, and $\sin ^{2} \theta_{23}=0.944$. Figure 6.16 compares the global minimum LED prediction to the data. The three-flavor prediction and the global minimum LED prediction are almost identical and the data is consistent with the three-flavor paradigm. It should be noted that the uncertainty bands for the LED predictions in Fig. 6.16 are constructed from the diagonal covariance matrix elements and do not include the correlations used in the actual fit. The next section discusses the interpretation of the sensitivity and data $\chi^{2}$ surfaces.

\subsection{Feldman-Cousins Data Limit}

This section discusses the global and FC interpretation of the sensitivity and data $\chi^{2}$ surfaces in the two-detector analysis.

\subsubsection{Interpreting the Sensitivity and Data $\chi^{2}$ Surfaces}

Figure 6.17 shows the global 90\% C.L. sensitivity coverages obtained for the CC and NC samples separately, the ND and FD samples separately, the individual samples, and for fixing the atmospheric parameters in the twodetector fit.

The top left panel of Fig. 6.17 shows that the sensitivity coverage due to $\mathrm{CC}$ events is dominant across the $\left(R, m_{0}\right)$ plane because of the higher purity and efficiency (in the FD) of the CC sample compared to the NC sample, as shown in Fig. 4.9. 

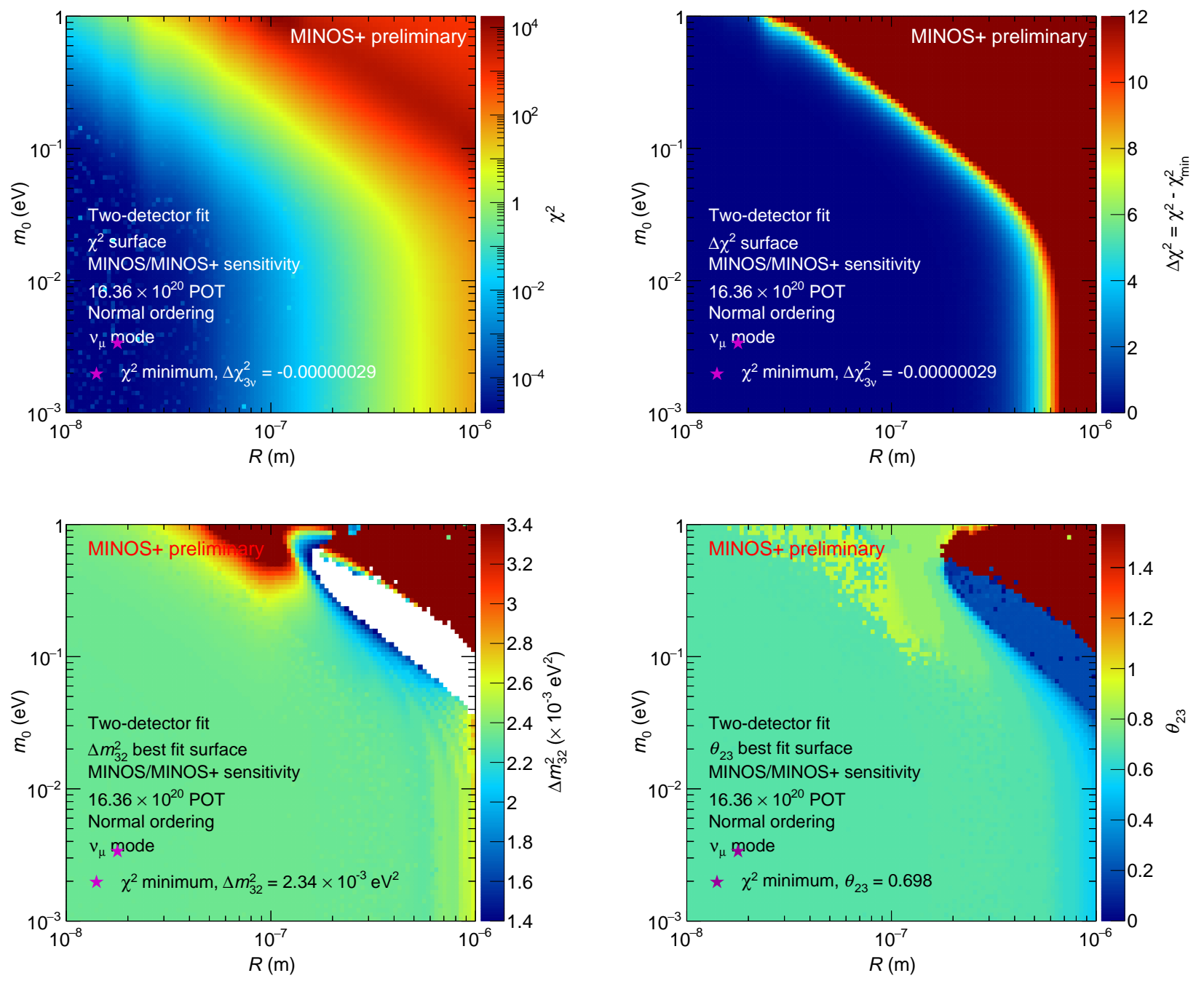

Figure 6.14: The sensitivity surfaces for the LED model in the $\left(R, m_{0}\right)$ plane for a simulated MINOS and MINOS+ exposure of $16.36 \times 10^{20}$ POT and assuming three-flavor simulated data: the $\chi^{2}$ surface (top left), the $\Delta \chi^{2}$ surface (top right), and the best fit values for $\Delta m_{32}^{2}$ (bottom left) and $\theta_{23}$ (bottom right). The white region in the bottom left panel corresponds to $\Delta m_{32}^{2}$ values between $10^{-6}$ and $1.4 \times 10^{-3} \mathrm{eV}^{2}$. 

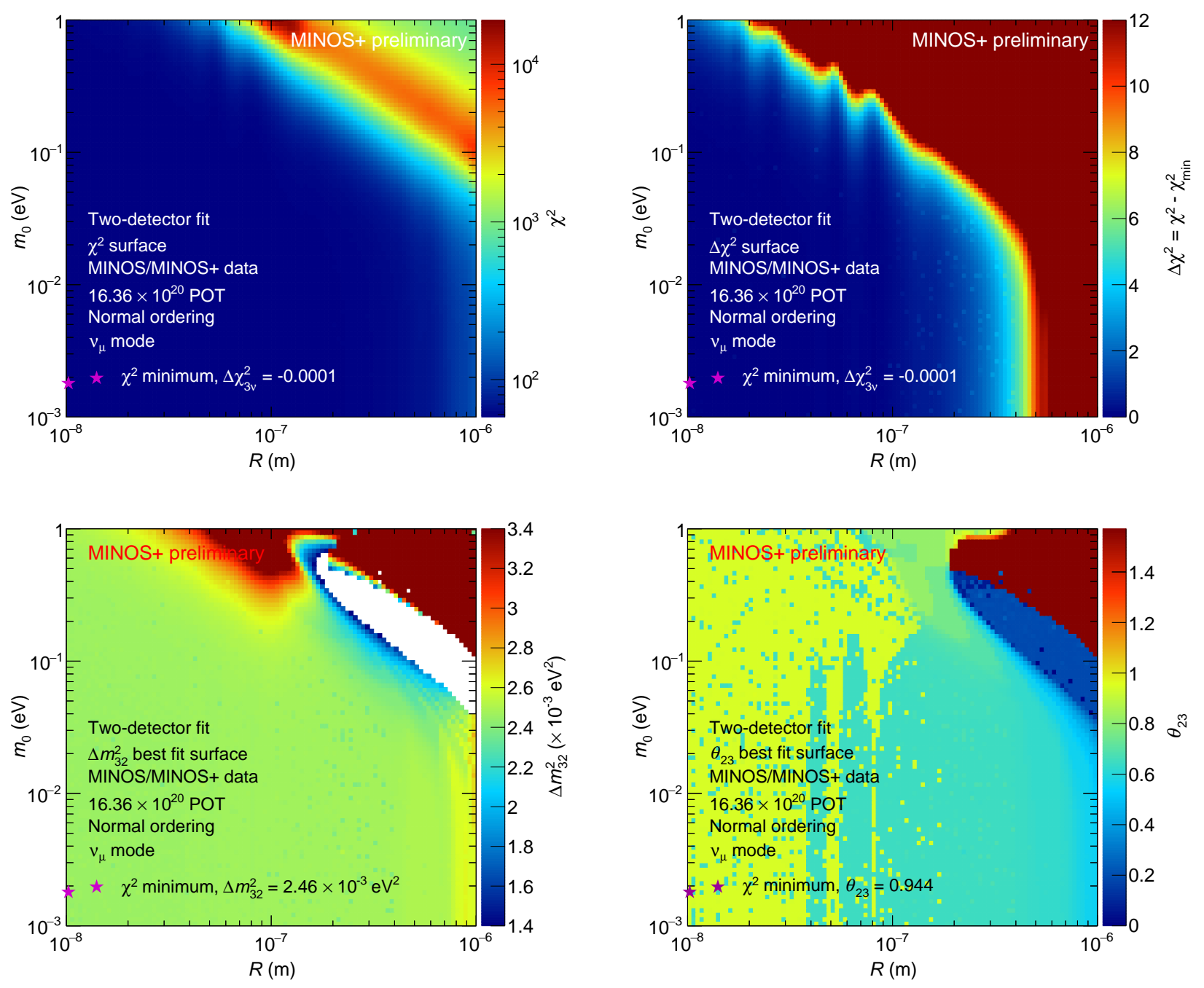

Figure 6.15: The data surfaces for the LED model in the $\left(R, m_{0}\right)$ plane for a MINOS and MINOS+ exposure of $16.36 \times 10^{20}$ POT: the $\chi^{2}$ surface (top left), the $\Delta \chi^{2}$ surface (top right), and the best fit values for $\Delta m_{32}^{2}$ (bottom left) and $\theta_{23}$ (bottom right). The white region in the bottom left panel corresponds to $\Delta m_{32}^{2}$ values between $10^{-6}$ and $1.4 \times 10^{-3} \mathrm{eV}^{2}$. 

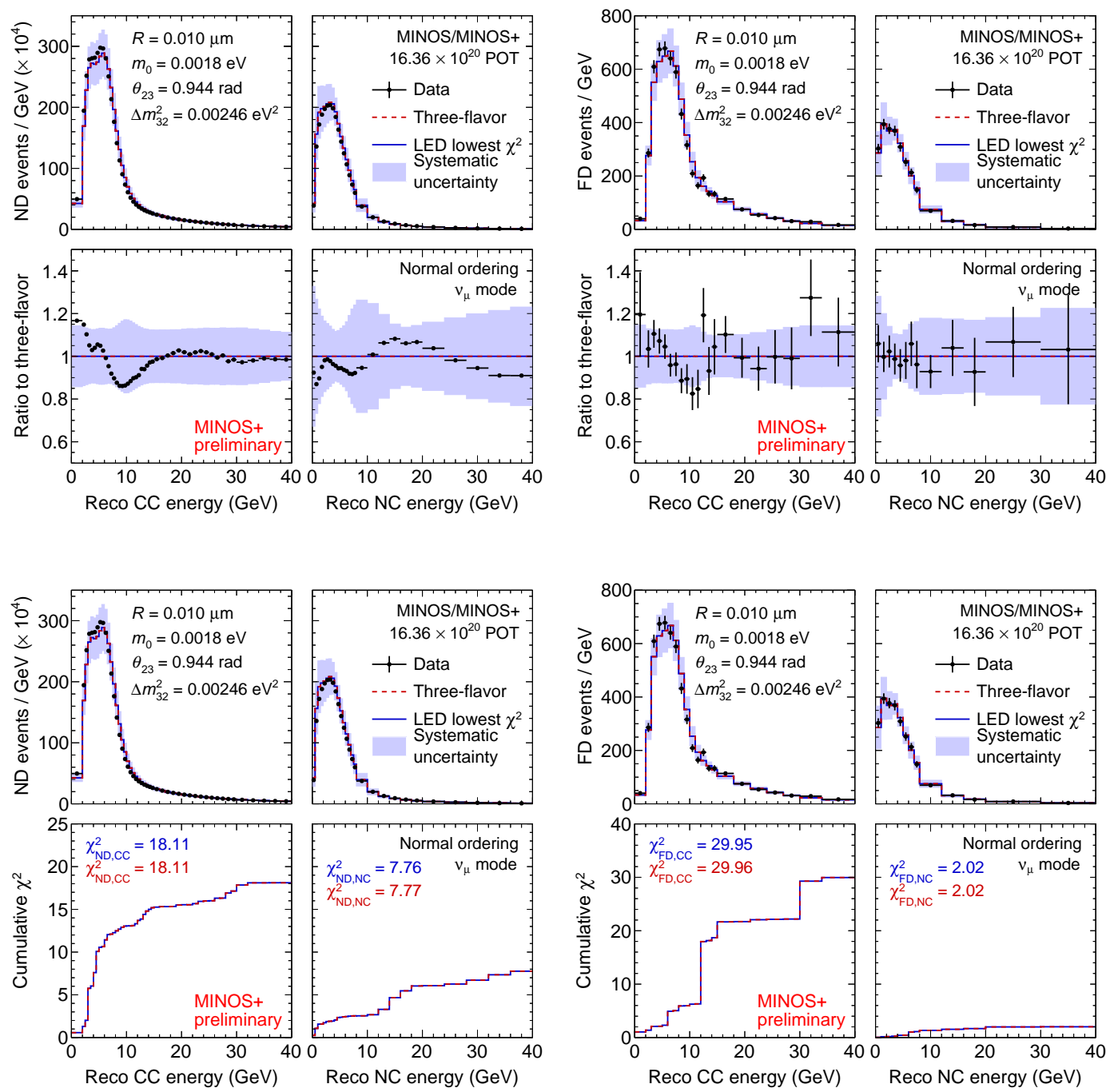

Figure 6.16: Comparison of the global minimum LED prediction (blue line) and three-flavor prediction (red line) to the data (black points) in the ND (left) and FD (right). The ratio of the data and global minimum LED prediction to the three-flavor prediction (top row) and the cumulative $\chi^{2}$ profiles of the global minimum LED and three-flavor predictions (bottom row) are also shown. It should be noted that the global minimum LED prediction is almost identical to the three-flavor prediction. 
The top right panel of Fig. 6.17 shows that for $m_{0} \gtrsim 0.2 \mathrm{eV}$ the sensitivity coverage is dominated by the ND predictions with the FD predictions providing a cross-check of the neutrino deficit, leading to a small increase in sensitivity when using the two-detector method. Below $0.2 \mathrm{eV}$, the FD sensitivity coverage dominates but significant improvement of the sensitivity coverage is obtained when employing the two-detector method due to the cross-checking mechanism between both detectors and the accompanying cancellation of systematic uncertainty effects due to correlations between both detectors.

The bottom left panel of Fig. 6.17 shows the constraining power of the individual ND CC, ND NC, FD CC, and FD NC samples.

Allowing the atmospheric parameters $\Delta m_{32}^{2}$ and $\theta_{23}$ to vary in the fit only has a small effect on the global $90 \%$ C.L. sensitivity and data coverages, as shown in the bottom right panel of Fig. 6.17.

The top left panel of Fig. 6.18 shows the effect of the individual systematic uncertainties on the global $90 \%$ C.L. sensitivity coverage. The largest reductions in sensitivity are caused by the cross section, normalization, and hadron production uncertainties. As mentioned in Section 6.2.5, the energy scale uncertainties are a dominant contribution to the total systematic uncertainty, but the reduction in sensitivity coverage is limited due to significant correlations between energy bins within each detector and between both detectors. Note that the global 90\% C.L. sensitivity coverage obtained with statistical uncertainties only is hidden underneath the global $90 \%$ C.L. sensitivity coverage obtained with the run period weight uncertainty only. 
The top right panel of Fig. 6.18 shows the global 90\% C.L. sensitivity coverages obtained when assuming simulated data with $R$ and $m_{0}$ values in different regions of the $\left(R, m_{0}\right)$ plane. The stars indicate the locations of the true $R$ and $m_{0}$ values assumed for these simulated datasets. The three-flavor values listed in Table 5.1 are assumed for the atmospheric parameters when generating the simulated data. The Asimov sensitivity coverage is shown in red for reference.

A comparison of the Far-over-Near and two-detector global 90\% C.L sensitivity coverages is shown in the bottom left panel of Fig. 6.18 for the MINOS only and MINOS/MINOS+ cases. Including the MINOS+ sample, with an increase of events at higher energies where the effects of LED are more visible in the FD, improves the experimental sensitivity at lower values of $m_{0}$, the region where the FD provides the dominant coverage. This is true for both analysis methods. When comparing the two-detector sensitivity coverage to the Far-over-Near sensitivity coverage, an overall improvement is observed due to better dynamics between the two detector predictions at a fixed point in LED parameter space. At higher values of $m_{0}$, where significant oscillations occur in the ND, the two-detector fit does not suffer from the weak constraints placed on the ND predictions in the Far-over-Near fit (see Formula (5.2)), while for lower values of $m_{0}$, the constraint of no oscillations in the ND also improves the sensitivity coverage.

Finally, a comparison of the raw, global, and FC 90\% C.L. data coverages is shown in the bottom right panel of Fig. 6.18. At low values of $m_{0}$, the 
raw and FC coverages are more constraining than the global coverage, while at high values of $m_{0}$, the FC coverage is less constraining than the raw and global coverages. The FC study used to obtain the FC data coverage is discussed in Section 6.4.2. In the limit of a vanishing lightest neutrino mass, the large extra dimension size is constrained to be smaller than $0.30 \mu \mathrm{m}$ at $90 \%$ C.L. by the MINOS/MINOS+ two-detector analysis. The construction of $1 \sigma$ and $2 \sigma$ sensitivity bands is discussed in Section 6.4.3.

\subsubsection{Feldman-Cousins Study}

Figure 6.19 shows the $90 \%$ up value surface of the $\Delta \chi_{\mathrm{FC}}^{2}$ distributions used to construct the MINOS/MINOS+ two-detector FC 90\% C.L. data coverage in the bottom right panel of Fig. 6.18.

A similar procedure as the one presented in Section 5.4.2 is employed to obtain the $\Delta \chi_{\mathrm{FC}}^{2}$ distributions. In the Far-over-Near analysis, the best fit values for $\Delta m_{32}^{2}$ and $\theta_{23}$ obtained from the data fit are adopted as true values for the fluctuated simulated data at selected points in the $\left(R, m_{0}\right)$ plane. For the two-detector fit MINOS/MINOS+ FC study, no information from the data fit is employed. Instead, the values listed in Table 5.1 are used for the atmospheric parameters. To avoid a possible bias from setting the atmospheric parameter values equal to those listed in Table 5.1 when simulating the data at any given point in the $\left(R, m_{0}\right)$ plane, the values of the atmospheric parameters are drawn from Gaussian distributions centered around the mean values listed 

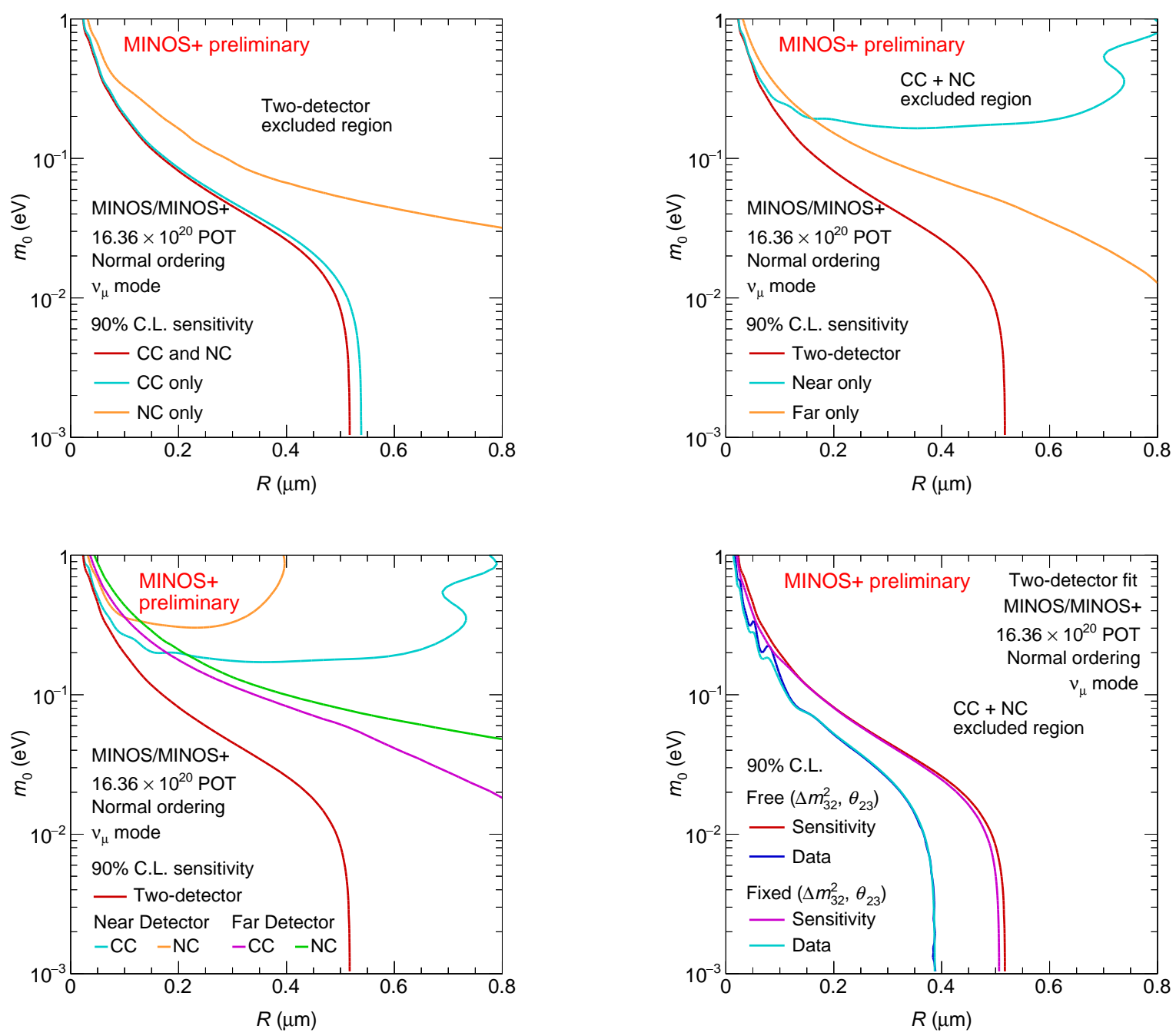

Figure 6.17: The effect of the individual samples and atmospheric parameters on the global $90 \%$ C.L. sensitivity coverages for a simulated MINOS and MINOS+ exposure of $16.36 \times 10^{20} \mathrm{POT}$ and assuming three-flavor simulated data: the CC versus NC samples (top left), a two-detector versus a singledetector fit (top right), the four individual samples (bottom left), and the effect of fixing the atmospheric parameters (bottom right). The bottom right panel also shows the comparison between the global 90\% C.L. sensitivity and data coverages. 

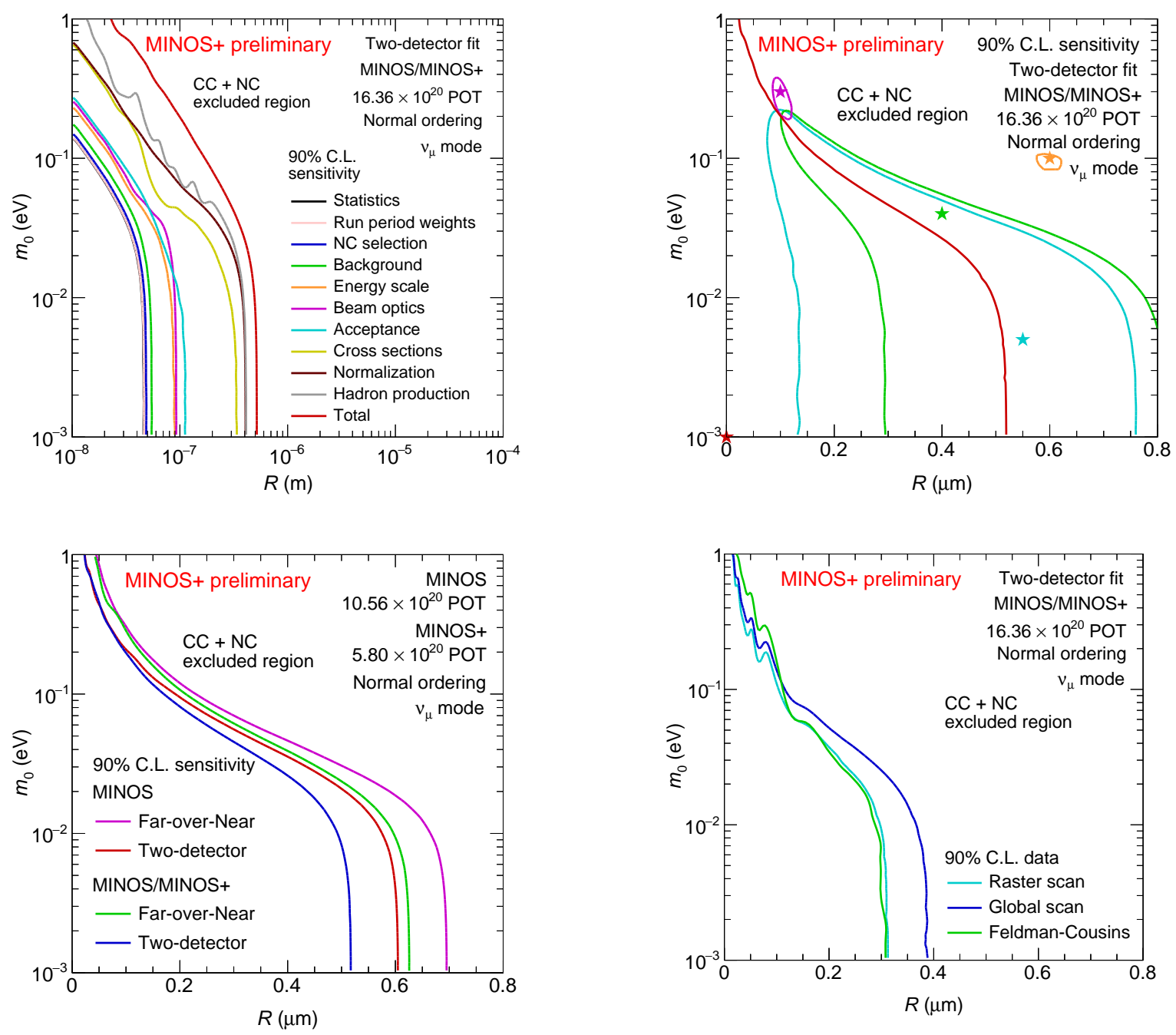

Figure 6.18: (Top left) The effect of the individual systematic uncertainties on the global $90 \%$ C.L. sensitivity coverage for a simulated MINOS and MINOS + exposure of $16.36 \times 10^{20}$ POT and assuming three-flavor simulated data. (Top right) Global 90\% C.L. sensitivity coverages assuming four different simulated LED signals (indicated by the stars). (Bottom left) Comparison of the two-detector global 90\% C.L. sensitivity coverages to their Far-over-Near counterparts. (Bottom right) Comparison of the two-detector raster, global, and Feldman-Cousins 90\% C.L. data coverages. 


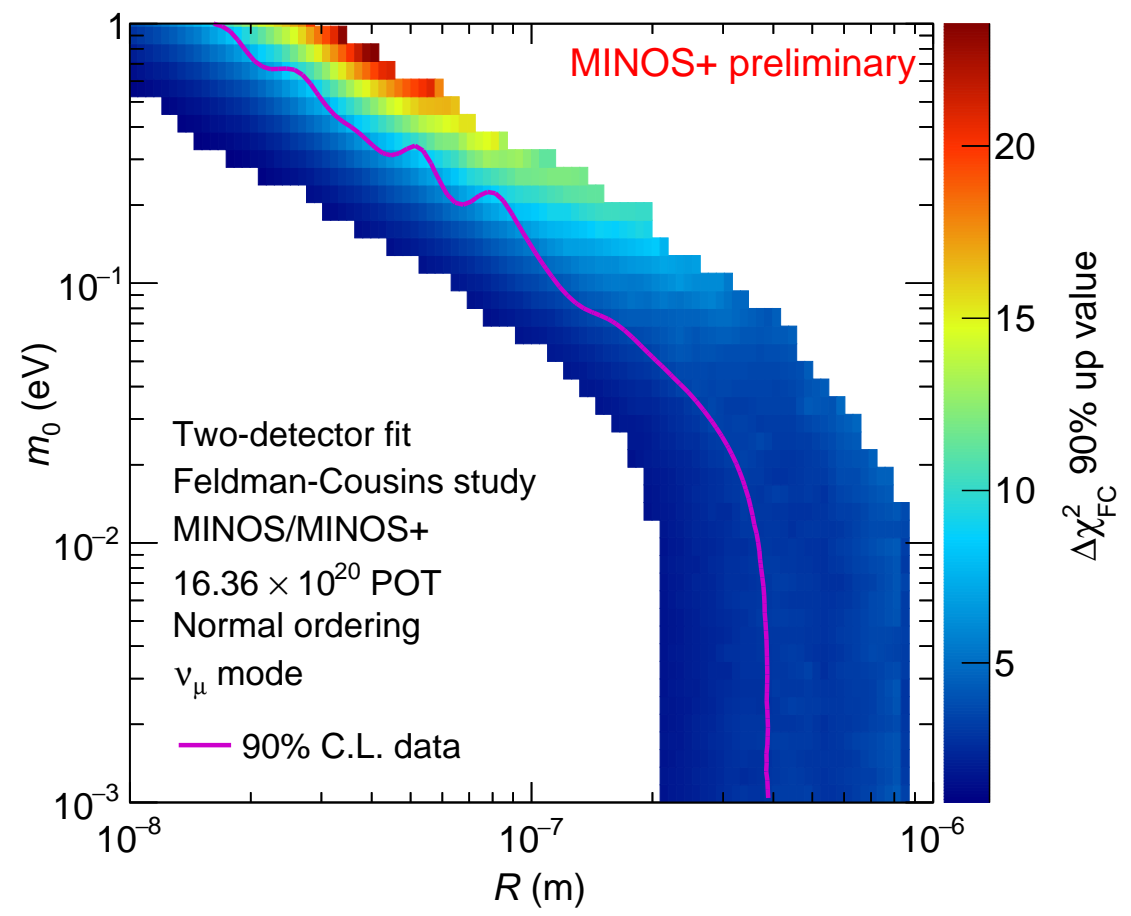

Figure 6.19: The $90 \%$ up value surface of the $\Delta \chi_{\mathrm{FC}}^{2}$ distributions used to construct the MINOS/MINOS+ two-detector Feldman-Cousins $90 \%$ C.L. data coverage and sensitivity bands in Fig. 6.23. The global 90\% C.L. data coverage (blue line) is shown for reference.

in Table 5.1 and with a standard deviation of $0.09 \times 10^{-3} \mathrm{eV}^{2}$ for $\Delta m_{32}^{2}$ and 0.040 for $\theta_{23}[30]$.

Figures 6.20 and 6.21 show the distributions for the two-detector FC study at two different points in the $\left(R, m_{0}\right)$ plane. The top left figure shows the $\Delta \chi_{\mathrm{FC}}^{2}$ distribution for 600 fluctuated simulated experiments. The green dashed line indicates the $90 \%$ up value for $\Delta \chi_{\mathrm{FC}}^{2}$, meaning that 540 of the fluctuated experiments have a smaller $\Delta \chi_{\mathrm{FC}}^{2}$ and 60 fluctuated experiments 
have a larger $\Delta \chi_{\mathrm{FC}}^{2}$. The middle and bottom row panels show the best fit

value distributions of $R, m_{0}, \Delta m_{32}^{2}$, and $\theta_{23}$ for the simulated experiments at this point. The distributions of true values for $\Delta m_{32}^{2}$ and $\theta_{23}$ around their nominal values from Table 5.1 are also shown.

\subsubsection{Constructing a Sensitivity Band}

It was already observed in the context of the Far-over-Near analysis in Section 5.4.3 that the median fluctuated sensitivity, obtained from a set of fluctuated sensitivities, provides a more representative estimate of the sensitivity to the LED model than the Asimov sensitivity. Figure 6.17 shows that a similar difference between the global 90\% C.L. Asimov sensitivity and data coverages is obtained in the two-detector analysis, and Appendix B studies this feature in more detail in the context of sterile neutrino oscillations in the $3+1$ model.

A set of 100 statistically and systematically fluctuated simulated threeflavor spectra is obtained to construct a $1 \sigma$ and $2 \sigma$ sensitivity bands in the $\left(R, m_{0}\right)$ plane. Fluctuated spectra of a sample are obtained by applying fluctuations to the Asimov three-flavor spectrum of that sample that consist of multivariate Gaussian fluctuations using a covariance matrix containing the three-flavor systematic uncertainties followed by statistical Poisson fluctuations. The multivariate Gaussian fluctuation procedure is outlined in Section 5.4.2 and a validation study in the context of the two-detector analysis is discussed in Appendix A. To avoid a possible bias from setting the atmo- 

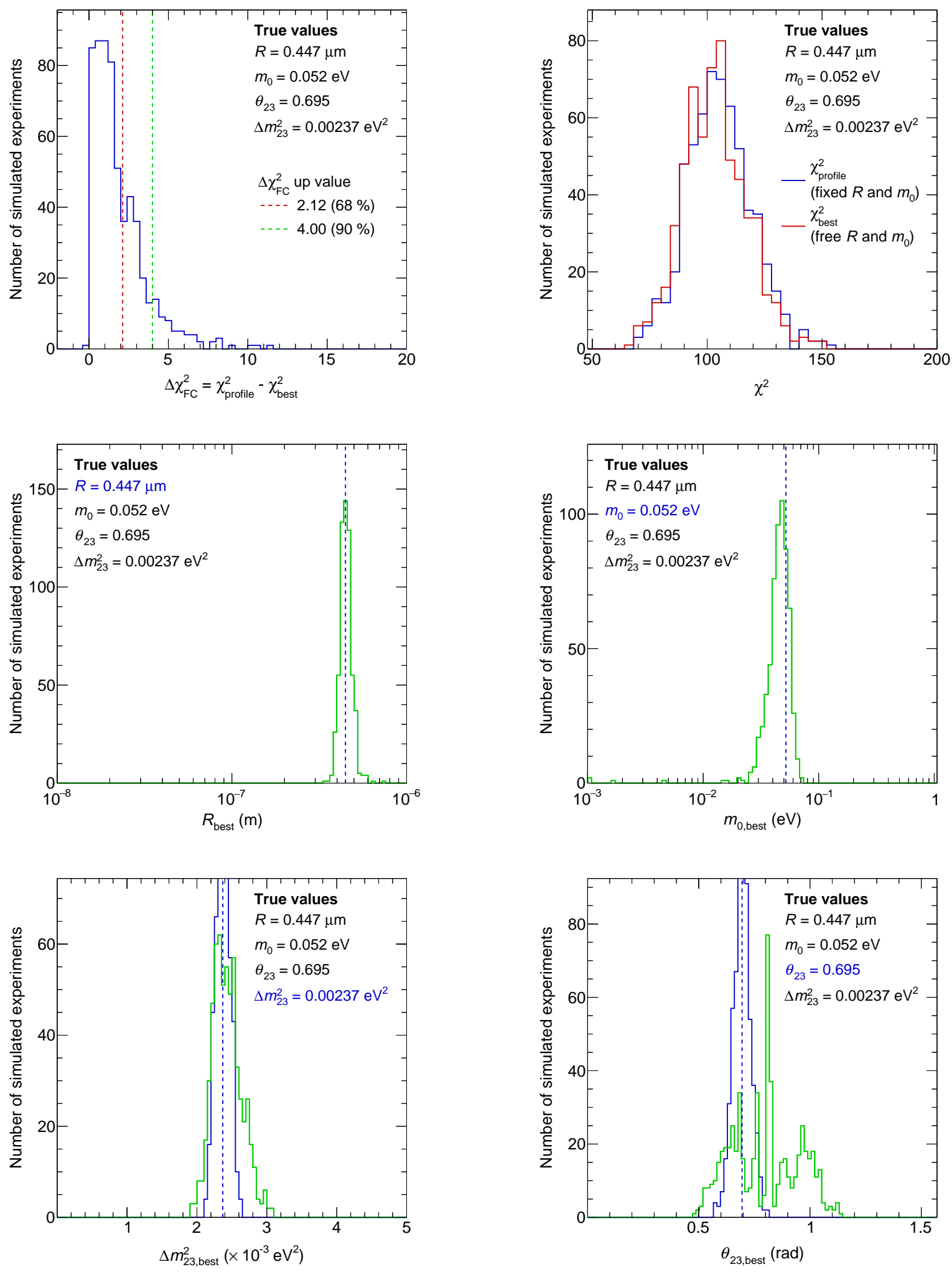

Figure 6.20: The $\Delta \chi_{\mathrm{FC}}^{2}$ and $\chi^{2}$ profiles (top rows) and the best fit distributions (middle and bottom rows) of the LED model parameters for 600 simulated experiments assuming the values $R=0.447 \mu \mathrm{m}, m_{0}=0.052 \mathrm{eV}$, and fluctuated values of the Asimov atmospheric parameters $\Delta m_{32}^{2}=2.37 \times 10^{-3} \mathrm{eV}^{2}$, and $\theta_{23}=0.695$. 

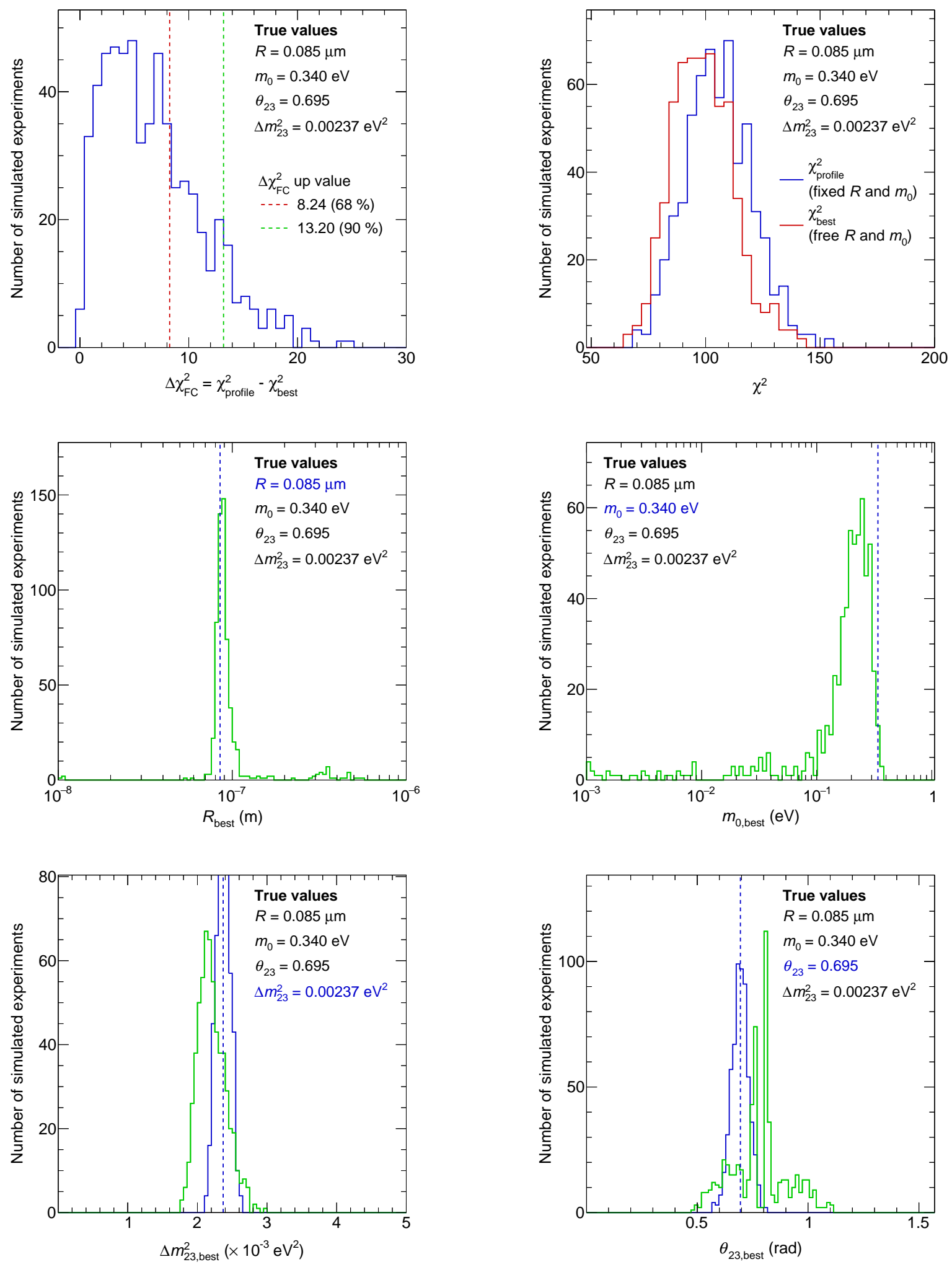

Figure 6.21: The $\Delta \chi_{\mathrm{FC}}^{2}$ and $\chi^{2}$ profiles (top rows) and the best fit distributions (middle and bottom rows) of the LED model parameters for 600 simulated experiments assuming the values $R=0.085 \mu \mathrm{m}, m_{0}=0.340 \mathrm{eV}$, and fluctuated values of the Asimov atmospheric parameters $\Delta m_{32}^{2}=2.37 \times 10^{-3} \mathrm{eV}^{2}$, and $\theta_{23}=0.695$. 
spheric parameter values equal to those listed in Table 5.1 when simulating the Asimov three-flavor spectrum, the values of the atmospheric parameters are drawn from Gaussian distributions centered around the mean values listed in Table 5.1 and with a standard deviation of $0.09 \times 10^{-3} \mathrm{eV}^{2}$ for $\Delta m_{32}^{2}$ and 0.040 for $\theta_{23}$ [30]. Figure 6.22 shows the 100 fluctuated simulated three-flavor spectra for the CC and NC samples in both detectors. Two example spectra are highlighted in cyan and magenta for illustrative purposes.

Due to high CPU requirements, only 100 fluctuated sensitivity $\chi^{2}$ surfaces are computed. It should be noted that in the context of the Far-over-Near analysis in Section 5.4.3, the atmospheric parameters were fixed, allowing to generate 1000 fluctuated sensitivity $\chi^{2}$ surfaces within a reasonable timespan. In the two-detector analysis, the atmospheric parameters are free in the fitting procedure used to obtain the fluctuated sensitivity $\chi^{2}$ surfaces, as is the case for the Asimov sensitivity and data $\chi^{2}$ surfaces.

The top panel in Fig. 6.23 shows the global 90\% C.L. coverages of the 100 fluctuated simulated three-flavor spectra shown in Fig. 6.22. The median of the fluctuated sensitivity coverages is shown in orange and is closer to the data coverage than the Asimov sensitivity coverage. At high $m_{0}$ values, the global $90 \%$ C.L. Asimov sensitivity coverage lies to the right of the data and fluctuated sensitivity coverages, a feature that is discussed in more detail in Appendix B in the context of the sterile $3+1$ model. 

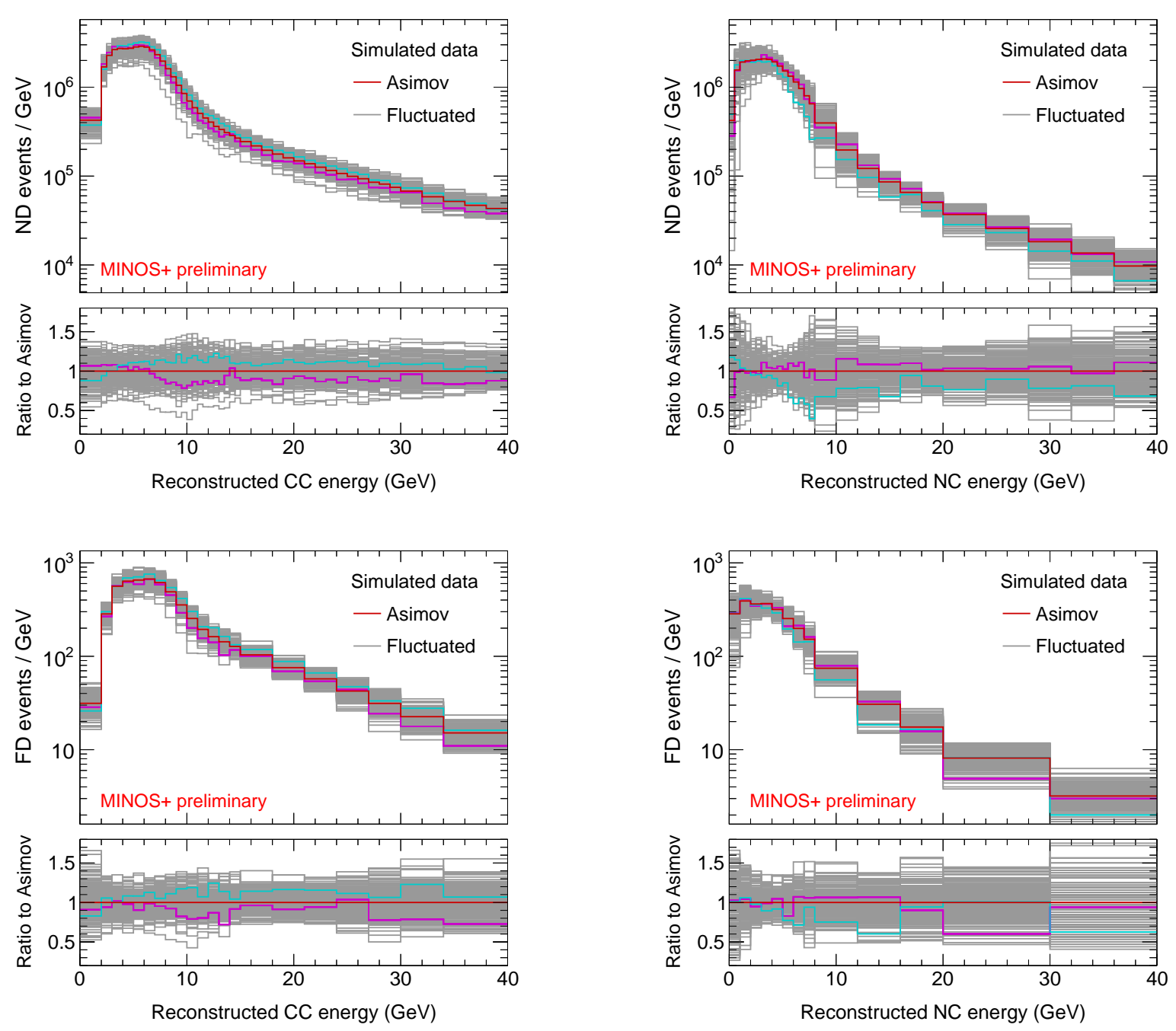

Figure 6.22: A set of 100 statistically and systematically fluctuated simulated three-flavor spectra used to construct the $1 \sigma$ and $2 \sigma$ sensitivity bands in the $\left(R, m_{0}\right)$ plane shown in Fig. 6.23. The ND CC (top left), ND NC (top right), FD CC (bottom left), and FD NC (bottom right) spectra are shown. The ratio of the fluctuated simulated spectra (gray lines) to the Asimov spectrum (red line) is shown for each sample. Two example fluctuated simulated spectra are highlighted in cyan and magenta for illustrative purposes. 
The bottom panel in Fig. 6.23 compares the FC 90\% C.L. data coverage to the $1 \sigma$ and $2 \sigma$ FC sensitivity bands. Below $m_{0}=0.2 \mathrm{eV}$, the FC $90 \%$ C.L. data coverage falls within the $1 \sigma$ sensitivity band.

Figure 6.24 shows the FC $90 \%$ C.L. data limit in the $\left(R, m_{0}\right)$ plane in terms of the effective mixing angle $\sin ^{2} \theta_{\text {eff }}^{\mu \mu}$ and the mass squared splitting $\Delta m_{\mathrm{KK}, 3}^{2}$, defined in Chapter 2. For $m_{0} \lesssim 0.1 \mathrm{eV}$, mixing effects due to the existence of large extra dimensions that are larger than about $2 \%$ are excluded at $90 \%$ C.L. by the MINOS/MINOS+ two-detector data. For $m_{0} \lesssim 0.01 \mathrm{eV}$, the mass squared splitting between the first KK mode and the active neutrino in the third tower, defined in Section 2.4.3, is constrained to be larger than about $0.5 \mathrm{eV}$ at $90 \%$ C.L. 

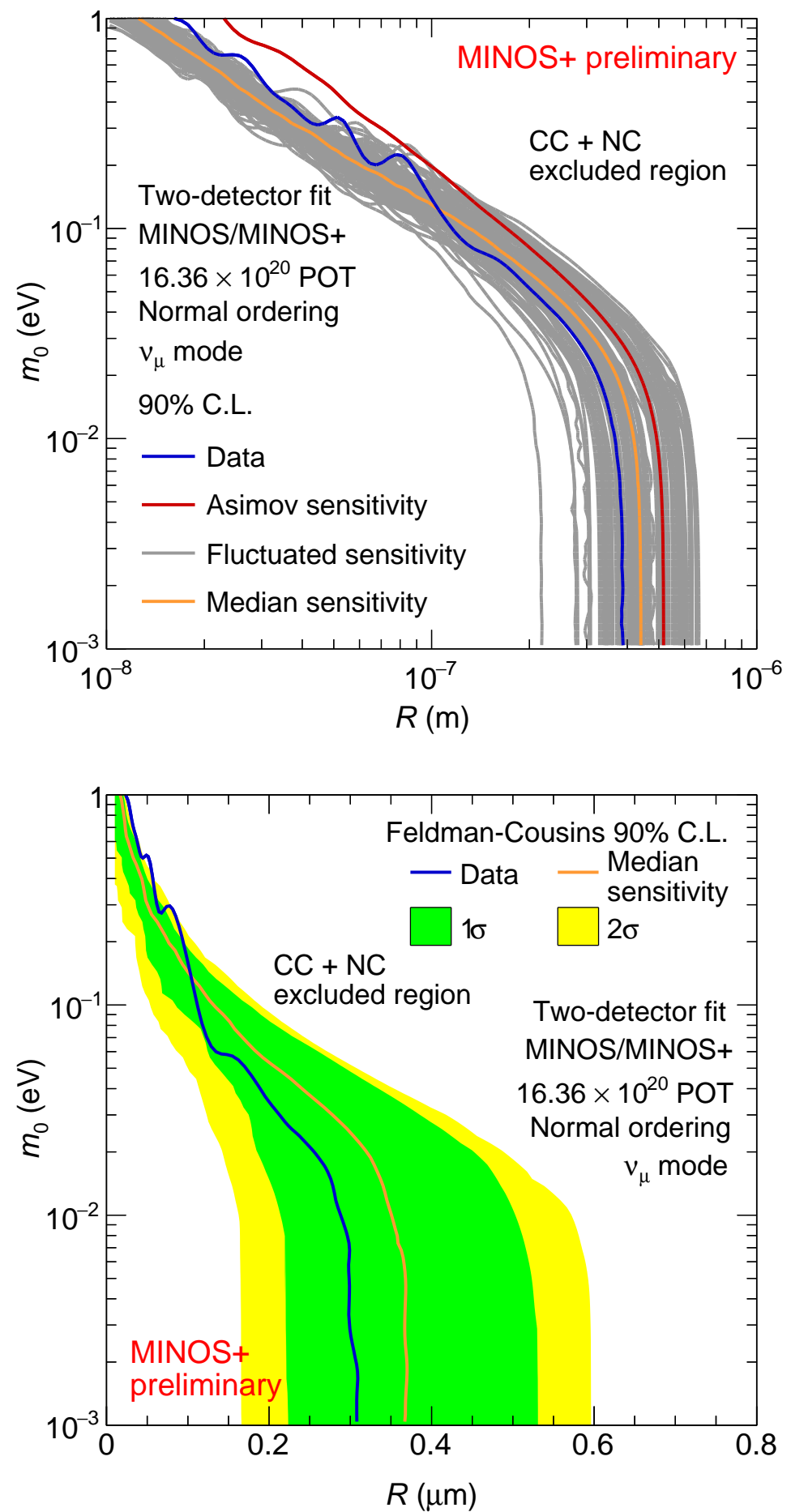

Figure 6.23: A fluctuated sensitivity study. (Top) A comparison of the MINOS/MINOS+ two-detector global 90\% C.L. Asimov (red line) and median fluctuated (orange line) sensitivity coverages. (Bottom) The MINOS/MINOS+ two-detector Feldman-Cousins $90 \%$ C.L. data coverage (blue line) is overlaid on the $1 \sigma$ and $2 \sigma$ sensitivity bands constructed from the Feldman-Cousins $90 \%$ C.L. interpretations of the $\Delta \chi^{2}$ surfaces that are used to obtain the global $90 \%$ C.L. fluctuated sensitivity coverages shown in the top panel. 

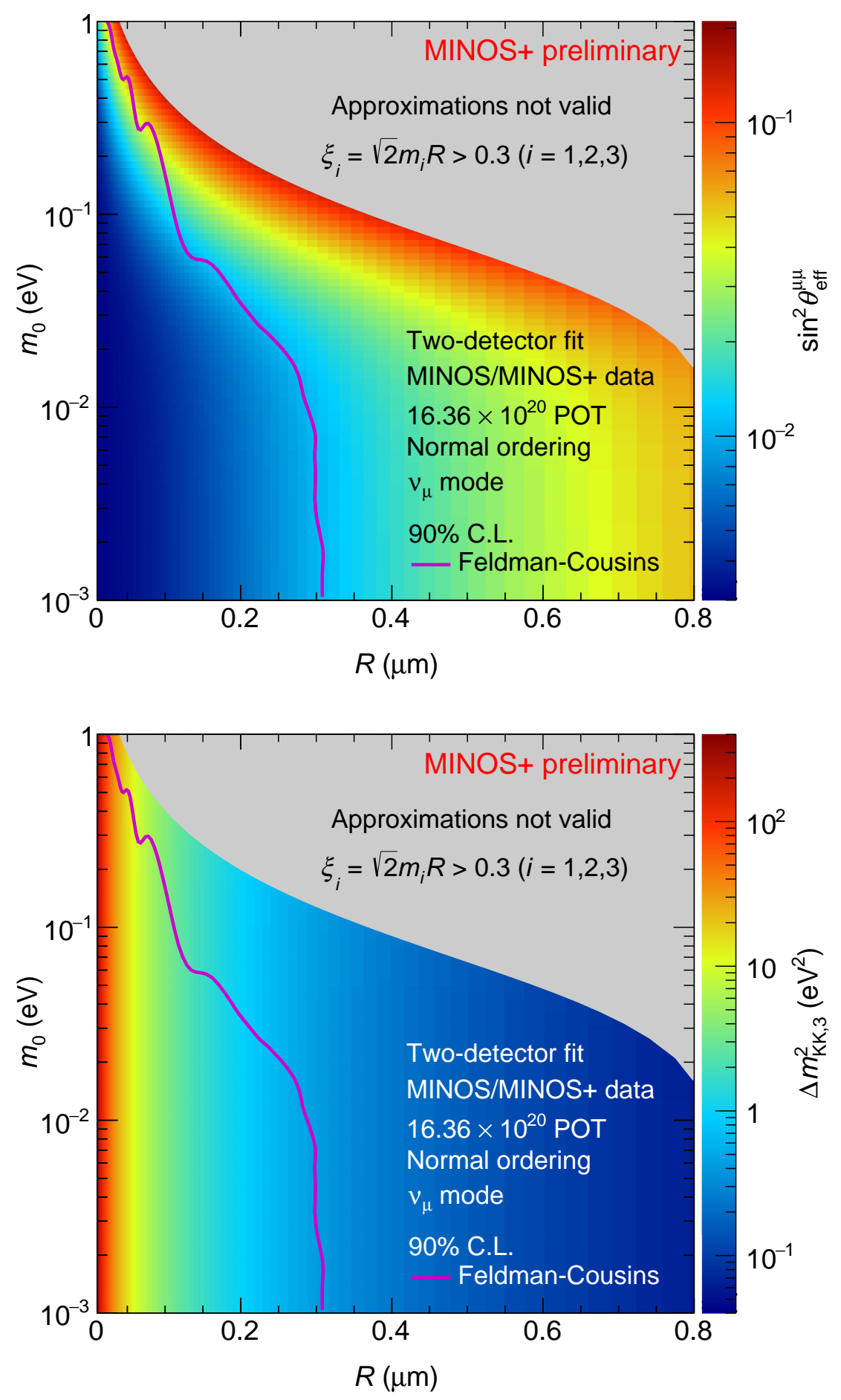

Figure 6.24: Interpreting the MINOS/MINOS+ two-detector FeldmanCousins $90 \%$ C.L. data limit in the $\left(R, m_{0}\right)$ plane in terms of the effective mixing angle $\sin ^{2} \theta_{\mathrm{eff}}^{\mu \mu}$ (top) and the mass squared splitting $\Delta m_{\mathrm{KK}, 3}^{2}$ (bottom). 


\section{Chapter 7}

\section{Results and Outlook}

In an effort to search for extra spatial dimensions using MINOS and MINOS+ accelerator beam muon neutrino data, the Large Extra Dimensions (LED) model is tested. The extra dimensions manifest themselves in $3+1$ spacetime through mixing of the active neutrinos with sterile neutrinos arising as Kaluza-Klein states, thereby altering the neutrino oscillation probabilities. The LED model provides a way to search for physics beyond the Standard Model using neutrino oscillation measurements. In addition, it provides a natural explanation for small Dirac neutrino masses.

No evidence for large extra dimensions is found in this dissertation. Two analysis strategies are employed to constrain the LED model parameters. Using the ratio of the neutrino energy spectra measured in both MINOS detectors, the Far-over-Near ratio, the first limit on this model by a neutrino oscillation experiment was reported. In the limit of a vanishing lightest active neutrino mass, the extra dimension size is constrained to be smaller than $0.45 \mu \mathrm{m}$ at $90 \%$ C.L. Adding the first two years of MINOS+ accelerator beam muon neutrino data and employing a new analysis strategy, the two-detector

method, that simultaneously exploits all neutrino energy spectra measured in 
the MINOS detectors without taking their ratio, the extra dimension size is further constrained to be smaller than $0.30 \mu \mathrm{m}$ at $90 \%$ C.L. Stronger limits are obtained for non-vanishing lightest neutrino masses. While this result is model-dependent, it is the strongest constraint on extra dimension sizes reported by a neutrino oscillation experiment and is very competitive compared to limits reported by tabletop gravitational experiments and collider experiments.

Stronger constraints on this model are expected when adding the final year of MINOS+ accelerator beam muon neutrino data to the analysis. Compared to MINOS data, the MINOS+ data correspond to higher neutrino energies, increasing the sensitivity to the LED model. Both the Far-over-Near and two-detector analysis methods can be employed by NOvA or the future DUNE experiment to provide additional constraints on this model that could be combined with the MINOS/MINOS+ result. An extended LED model, LED+, involving three additional bulk masses, can be tested using the large and rich dataset of MINOS and MINOS+. 
Appendices 


\section{Appendix A}

\section{Validating the Fluctuation Procedure}

Statistically and systematically fluctuated simulated data spectra are employed in the Far-over-Near and two-detector analyses to construct a sensitivity uncertainty band and to perform a Feldman-Cousins study.

For a given nominal spectrum (e.g. the Asimov three-flavor spectrum when constructing the sensitivity uncertainty band) and covariance matrix $V$, a fluctuated spectrum is obtained by applying a multivariate Gaussian fluctuation to the upper-triangular decomposition of $V$, as discussed in Section 5.4.2. This fluctuation procedure ensures that the fluctuated simulated data spectrum has bin-to-bin correlations that are consistent with the correlations in $V$. In the Far-over-Near analysis, both the statistical and systematic fluctuation are applied simultaneously through a decomposition of the combined covariance matrix $V=V_{\text {stat }}+V_{\text {syst }}$. In the two-detector analysis, a systematic

fluctuation through a decomposition of $V_{\text {syst }}$ is followed by a Poisson fluctuation of the resulting systematically fluctuated simulated data spectrum.

Two methods were used in this dissertation to perform the uppertriangular decomposition of $V$ [144]:

- a Cholesky decomposition in the Far-over-Near analysis, and 
- a Singular Value decomposition followed by a QR decomposition (SVD+QR) in the two-detector analysis.

This appendix discusses the results of a validation study of both techniques and shows that equivalent results are obtained.

\section{A.1 Reconstructing the Covariance Matrix from Fluc- tuated Simulated Data Spectra}

As a validation test, the covariance matrix is reconstructed from a set

of $N$ fluctuated simulated data spectra and compared with the original covariance matrix that was employed to obtain these fluctuated spectra. It should be noted that the original covariance matrix is constructed from uncertainty bands, as discussed in Section 5.2. As the nominal spectrum, the Asimov three-flavor simulated data is used. The $i j^{\text {th }}$ element of the reconstructed matrix $V_{\text {reco }}$ is given by

$$
V_{\text {reco }, i j}=\frac{1}{N} \sum_{\alpha=1}^{N} \frac{\left(f_{\alpha i}-n_{i}\right)\left(f_{\alpha j}-n_{j}\right)}{n_{i} n_{j}},
$$

where $f_{\alpha i}$ and $n_{i}$ are the number of events in bin $i$ of fluctuated spectrum $\alpha$ and the nominal spectrum, respectively.

\section{A.2 A Few Examples}

The validation tests presented in this section are in the context of the two-detector analysis. Figures A.1 through A.9 compare an original twodetector covariance matrix to the reconstructed covariance matrix obtained 
employing Formula (A.1) for four different two-detector systematic uncertainties: the full analysis systematic uncertainty in Fig. A.1 through Fig. A.3, the energy scale systematic uncertainties in Fig. A.4 and A.5, the hadron production systematic uncertainty in Fig. A.6 and A.7, and the cross section systematic uncertainty in Fig. A.8 and A.9. It should be noted that these matrices are not the final versions used in Chapter 6. However, for the purpose of this study that does not matter.

The bottom left panel of Fig. A.1 shows 30 example fluctuated simulated data spectra for the $\mathrm{CC}$ and $\mathrm{NC}$ samples in both the FD and ND obtained using a multivariate Gaussian fluctuation procedure employing the SVD+QR decomposition technique with the full analysis systematic covariance matrices shown in the top panels. The bottom right panel of Fig. A.1 shows the ratio of the fluctuated spectra to the nominal spectrum. A similar set of fluctuated data spectra is obtained employing the Cholesky decomposition. The multivariate Gaussian distribution ensures a symmetric distribution of fluctuated spectra w.r.t. the norminal spectrum. The top row panels of Fig. A.2 show the same $\mathrm{CC}$ and $\mathrm{NC}$ covariance matrix as in Fig. A.1, but in terms of energy bins rather than reconstructed energy. The middle and bottom row panels show the fractional difference of the original and reconstructed matrices for Cholesky and SVD+QR decomposition, respectively, obtained using Formula (A.1) for 10000 fluctuated simulated data spectra. With the exception of a few energy bins, the fractional difference is consistent with 0 for the $\mathrm{CC}$ sample. For the NC sample there are many more bins for which the fractional 
difference is significantly different from zero. These fractional differences different from 0 correspond to energy bins in the matrix where the covariance is very small and transitions from negative to positive covariances. Figure A.3 shows that the offset from zero disappears with increasing number of fluctuated spectra used in Formula (A.1). Comparing the fractional differences for the Cholesky and SVD+QR techniques in the middle and bottom rows of Fig. A.2, respectively, it is observed that for smaller number of fluctuated spectra the SVD+QR decomposition technique performs better. As such, the SVD+QR technique was adopted for the two-detector analysis presented in Chapter 6 . Figures A.4 through A.9 show the validation test results obtained with the SVD+QR decomposition technique for the energy scale, hadron production, and cross section systematic uncertainties. 

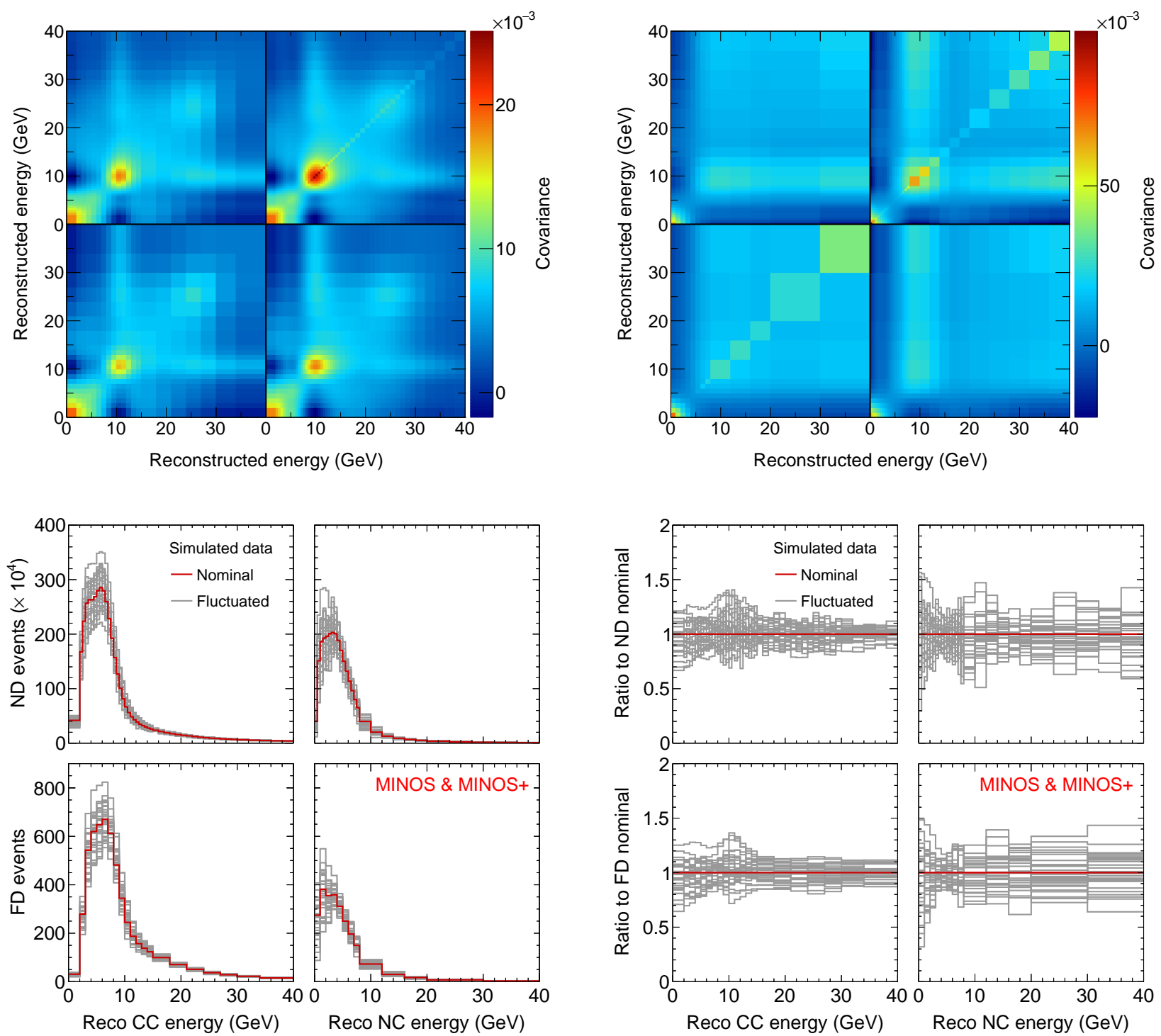

Figure A.1: Fluctuated simulated data spectra in the two-detector analysis. The full analysis systematic covariance matrices for the CC (top left) and NC (top right) samples used in the multivariate Gaussian fluctuation procedure through SVD+QR upper-triangular matrix decomposition. A set of 30 fluctuated spectra (bottom left) and their ratio to the nominal spectrum (bottom right) are shown for the four samples, ND CC, ND NC, FD CC, and FD NC. 

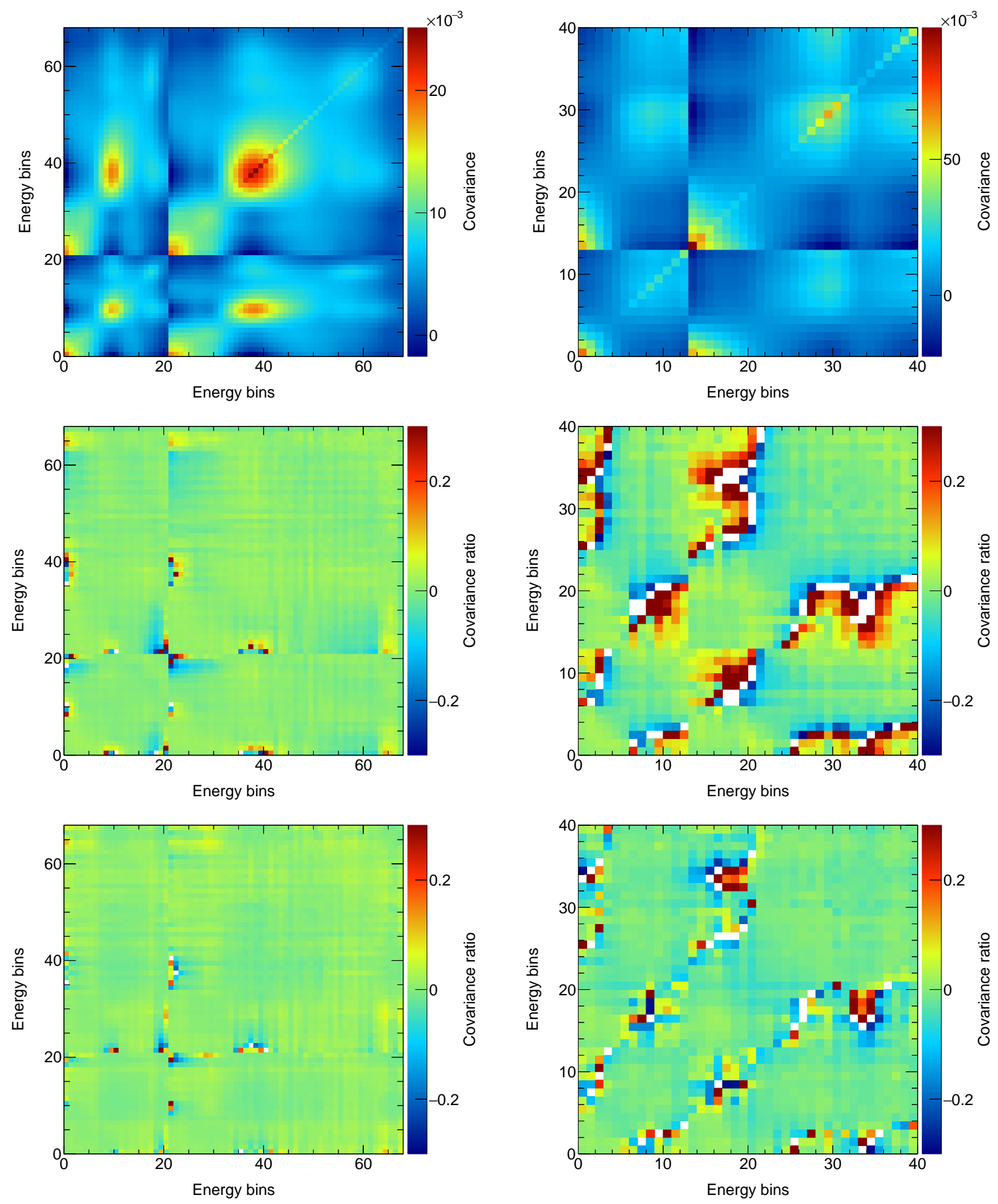

Figure A.2: The original matrices from Fig. A.1 (top row) and the fractional difference of the original and reconstructed matrices for the Cholesky (middle row) and SVD+QR (bottom row) decomposition obtained from 10000 fluctuated spectra. Unlike in Fig. A.1, the matrices are shown in terms of energy bins rather than reconstructed energy. The left (right) column shows the CC (NC) matrix. 

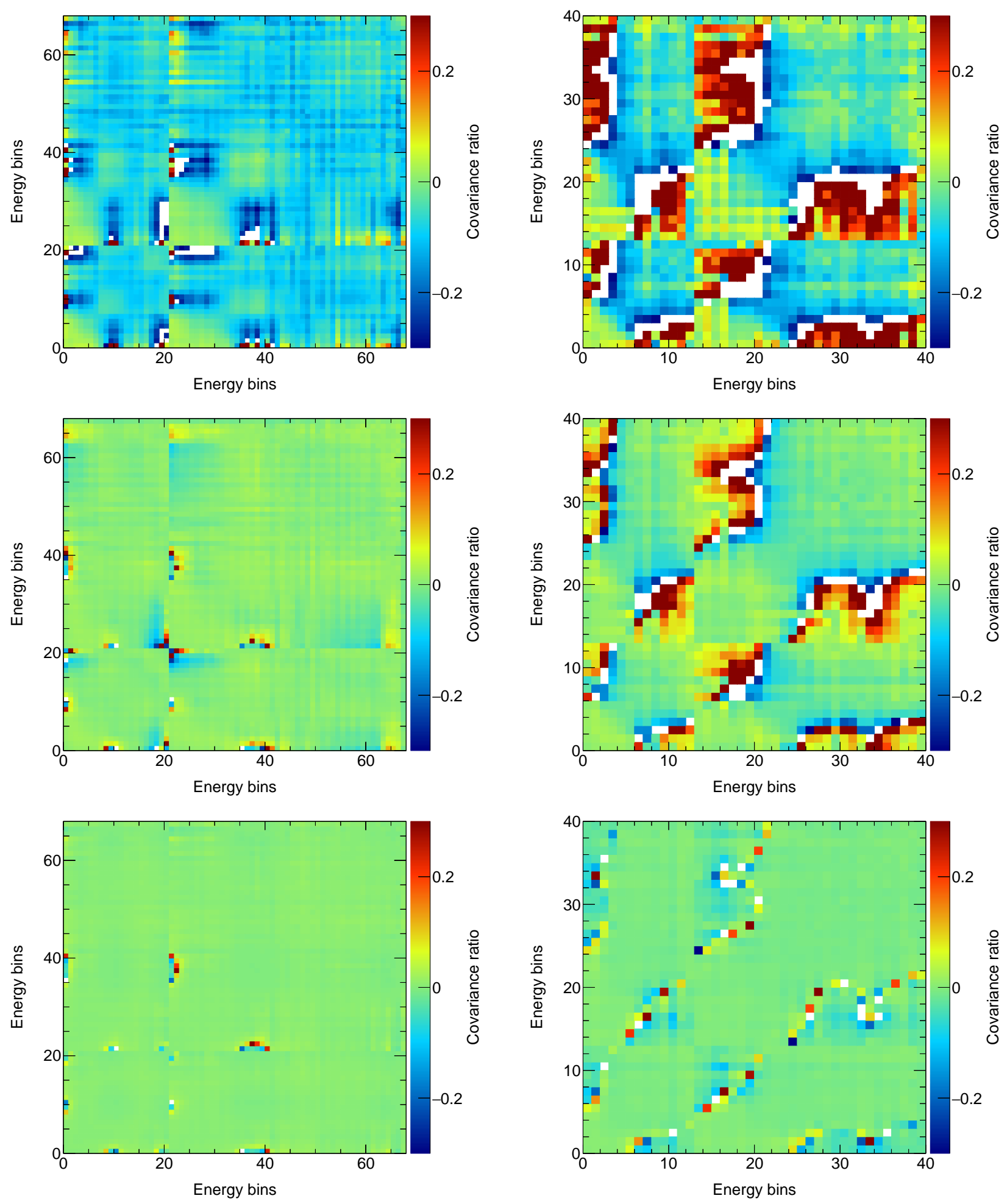

Figure A.3: The effect of increasing the number of fluctuated simulated data spectra on the fractional difference between the original and reconstructed full analysis systematic covariance matrix for the Cholesky decomposition: 1000 spectra (top row), 10000 spectra (middle row), 100000 spectra (bottom row), with the left (right) column showing the $\mathrm{CC}(\mathrm{NC})$ matrix. 

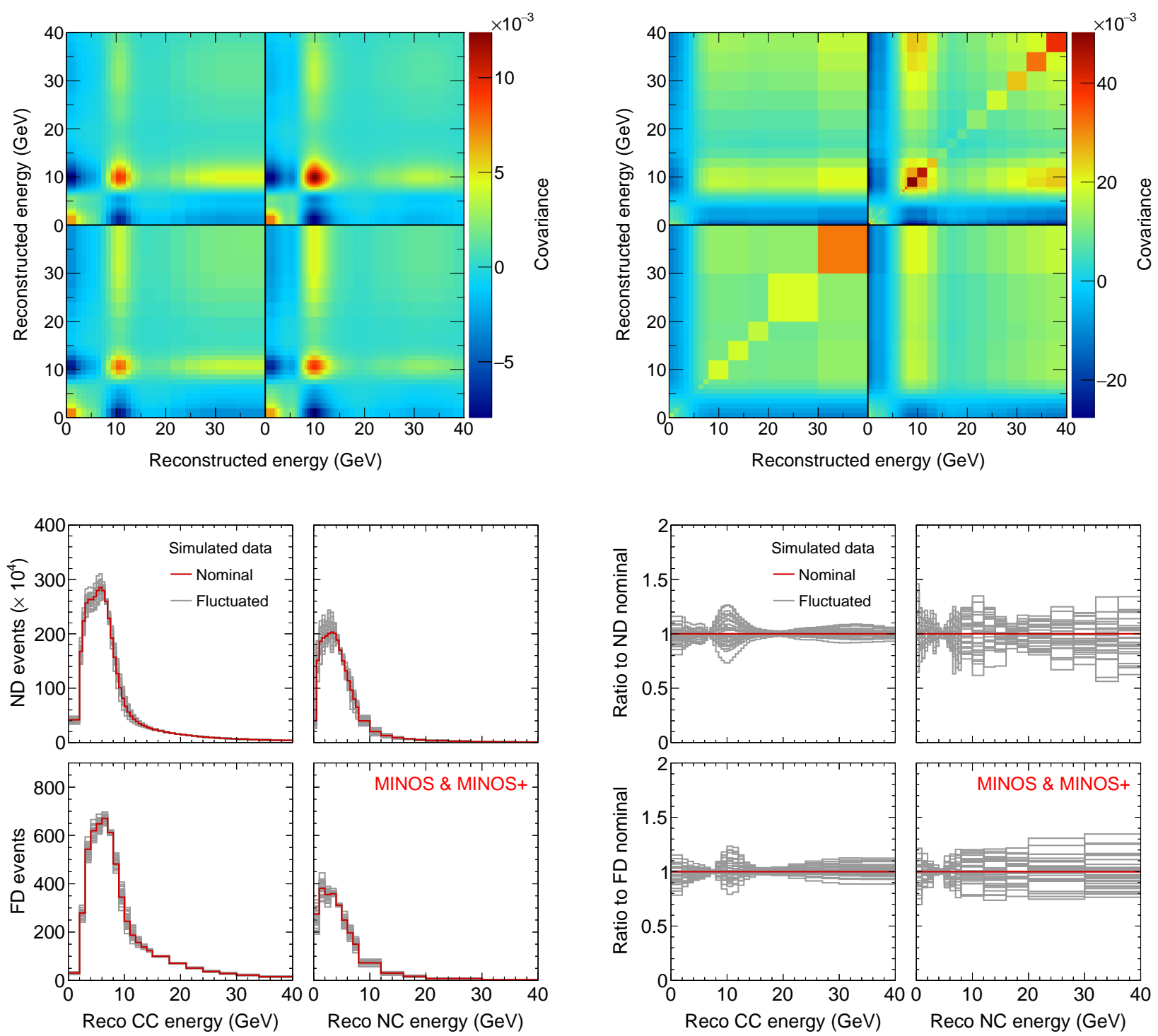

Figure A.4: Fluctuated simulated data spectra in the two-detector analysis. The energy scale systematic covariance matrices for the CC (top left) and NC (top right) samples used in the multivariate Gaussian fluctuation procedure through SVD+QR upper-triangular matrix decomposition. A set of 30 fluctuated spectra (bottom left) and their ratio to the nominal spectrum (bottom right) are shown for the four samples, ND CC, ND NC, FD CC, and FD NC. 

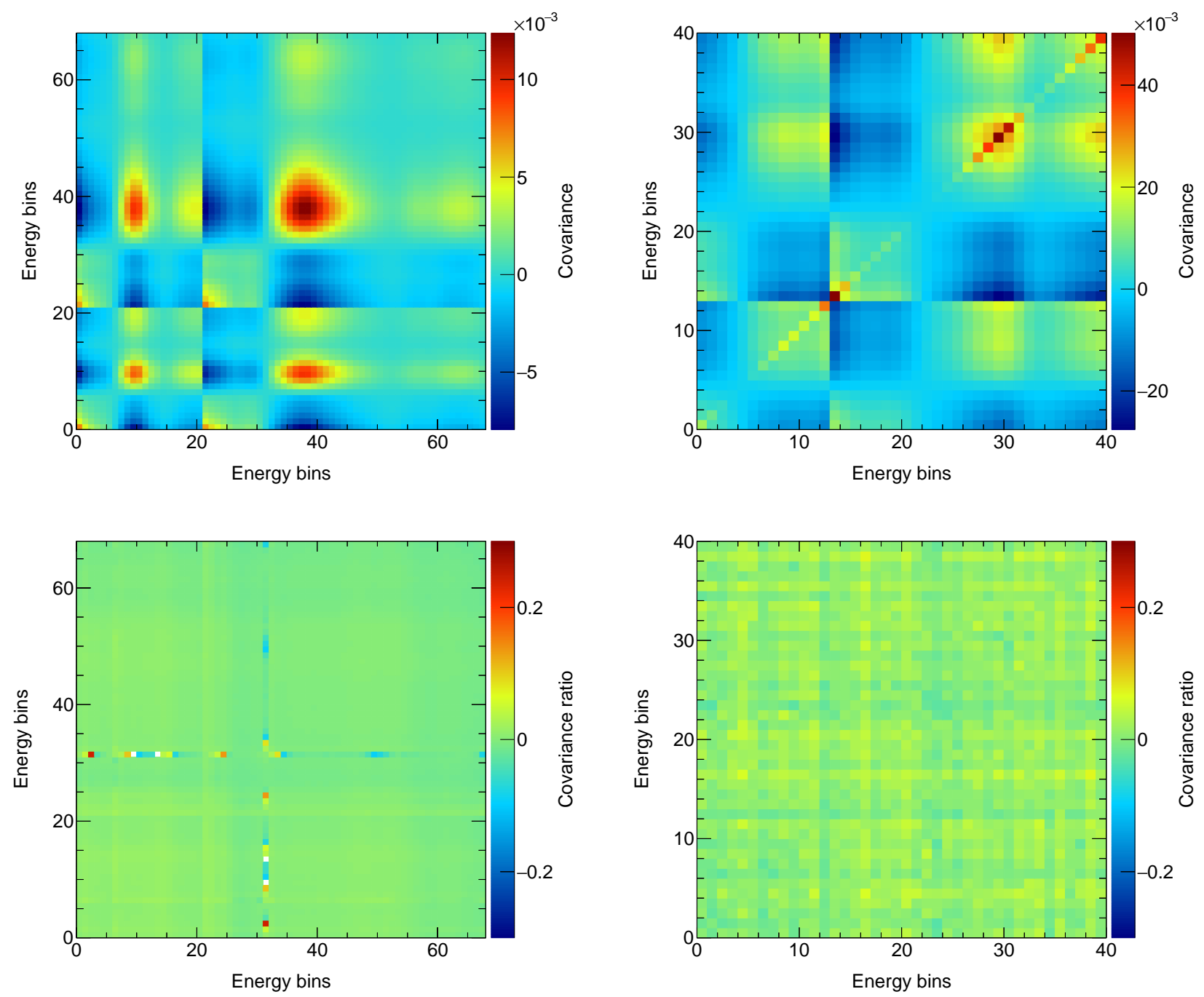

Figure A.5: The original matrices from Fig. A.4 (top row) and the fractional difference of the original and reconstructed matrices for the SVD+QR (bottom row) decomposition obtained from 10000 fluctuated spectra. Unlike in Fig. A.4, the matrices are shown in terms of energy bins rather than reconstructed energy. The left (right) column shows the CC (NC) matrix. 

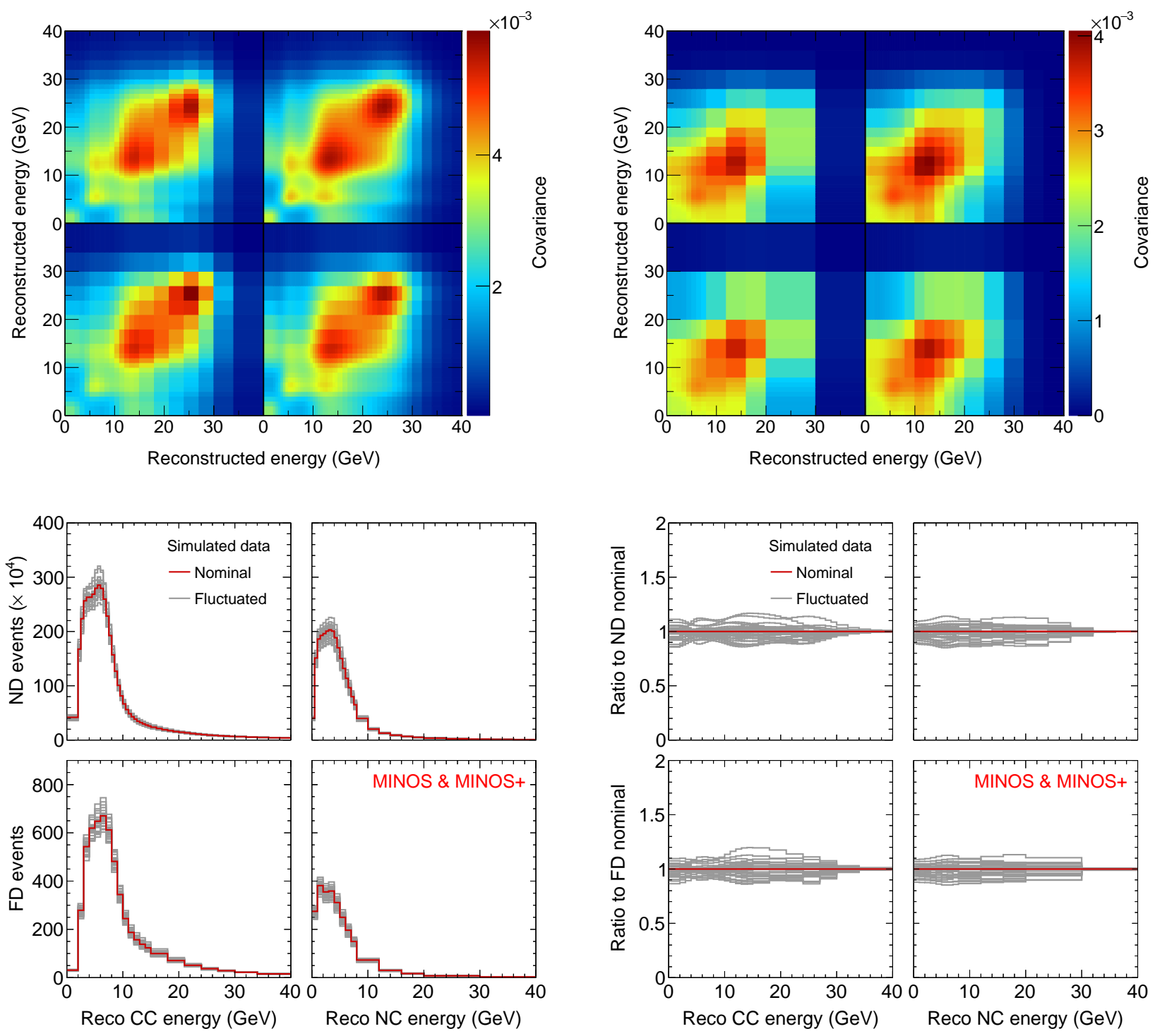

Figure A.6: Fluctuated simulated data spectra in the two-detector analysis. The hadron production systematic covariance matrices for the CC (top left) and NC (top right) samples used in the multivariate Gaussian fluctuation procedure through SVD+QR upper-triangular matrix decomposition. A set of 30 fluctuated spectra (bottom left) and their ratio to the nominal spectrum (bottom right) are shown for the four samples, ND CC, ND NC, FD CC, and FD NC. 

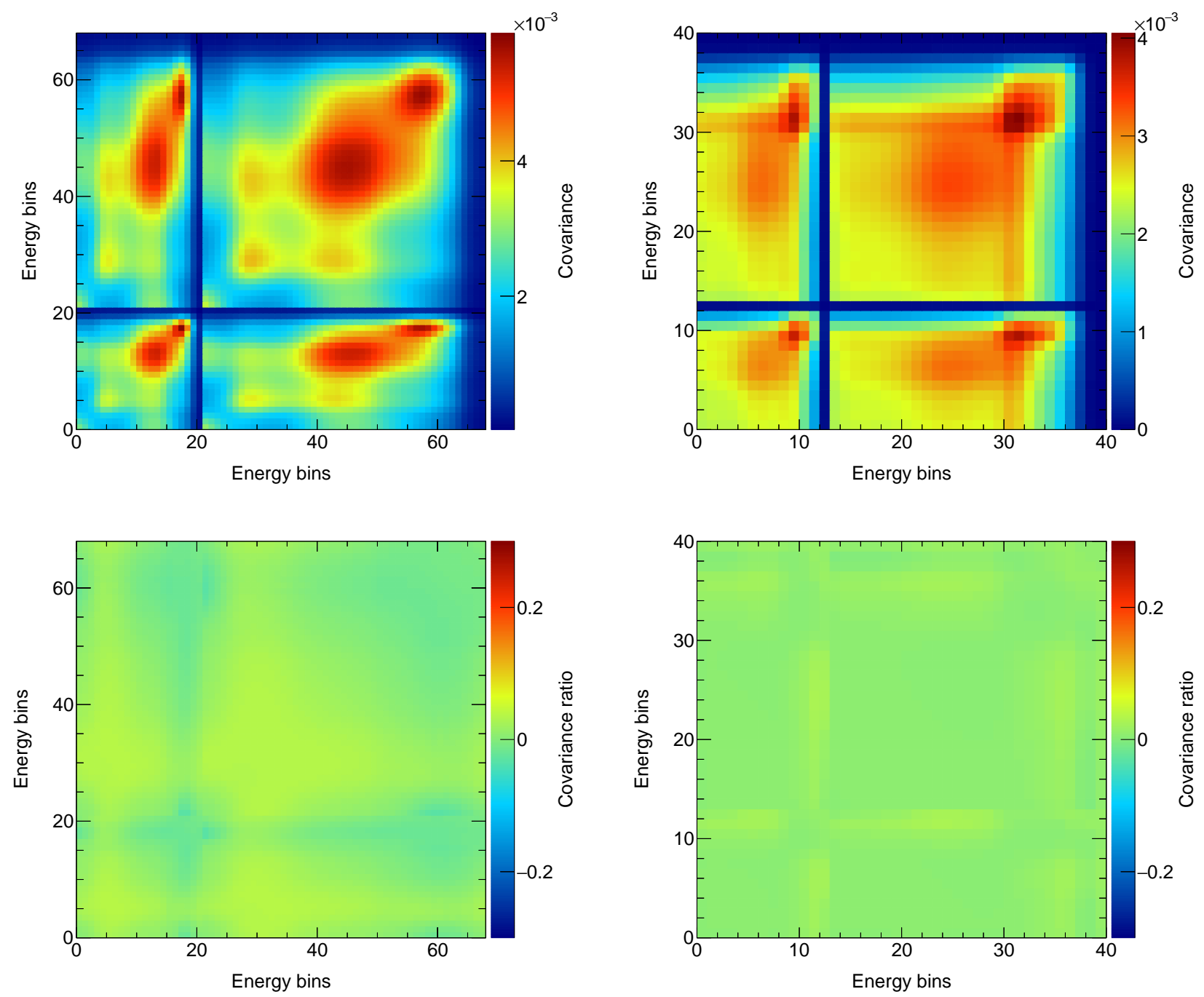

Figure A.7: The original matrices from Fig. A.6 (top row) and the fractional difference of the original and reconstructed matrices for the SVD+QR (bottom row) decomposition obtained from 10000 fluctuated spectra. Unlike in Fig. A.6, the matrices are shown in terms of energy bins rather than reconstructed energy. The left (right) column shows the CC (NC) matrix. 

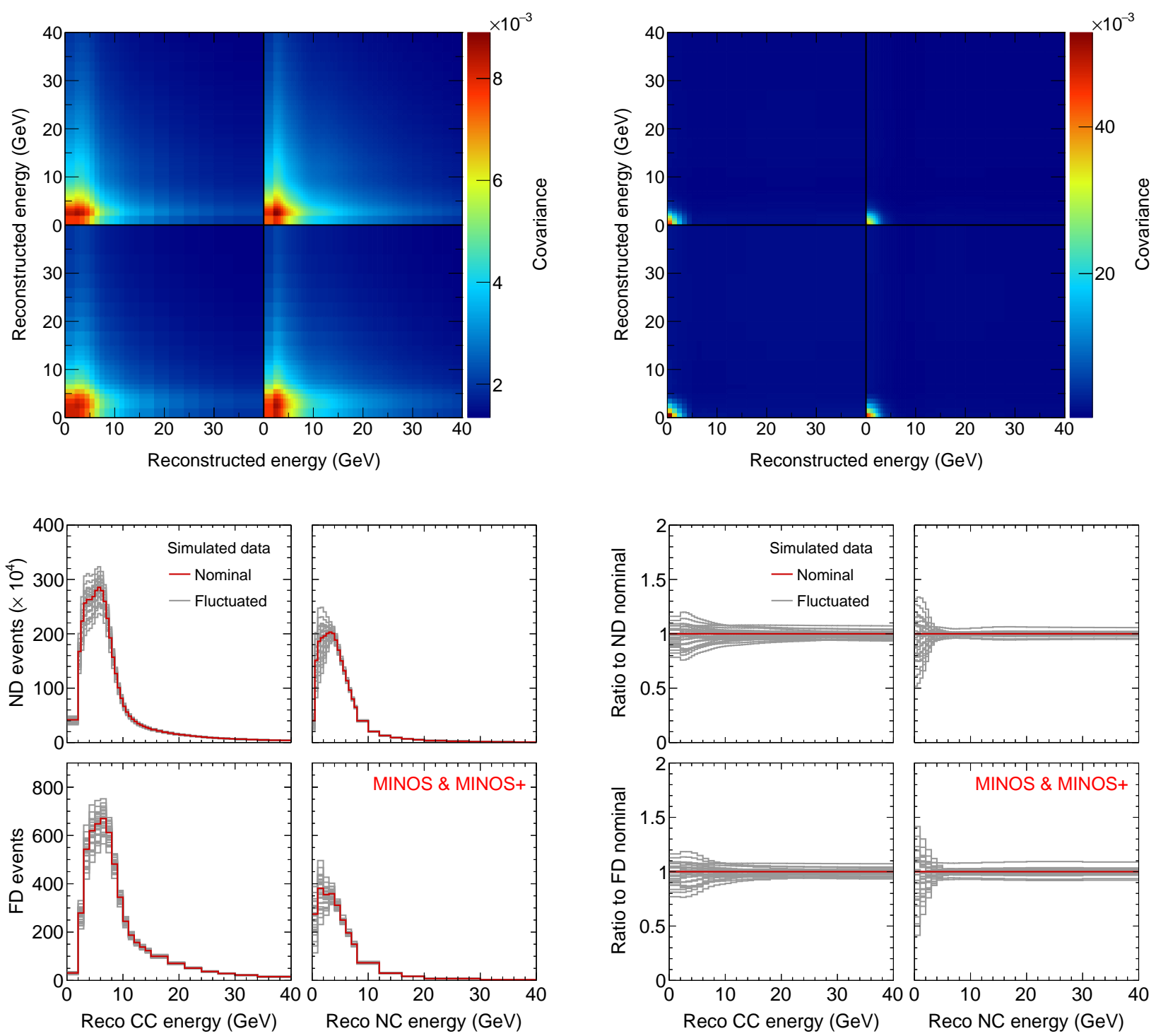

Figure A.8: Fluctuated simulated data spectra in the two-detector analysis. The cross section systematic covariance matrices for the CC (top left) and NC (top right) samples used in the multivariate Gaussian fluctuation procedure through SVD+QR upper-triangular matrix decomposition. A set of 30 fluctuated spectra (bottom left) and their ratio to the nominal spectrum (bottom right) are shown for the four samples, ND CC, ND NC, FD CC, and FD NC. 

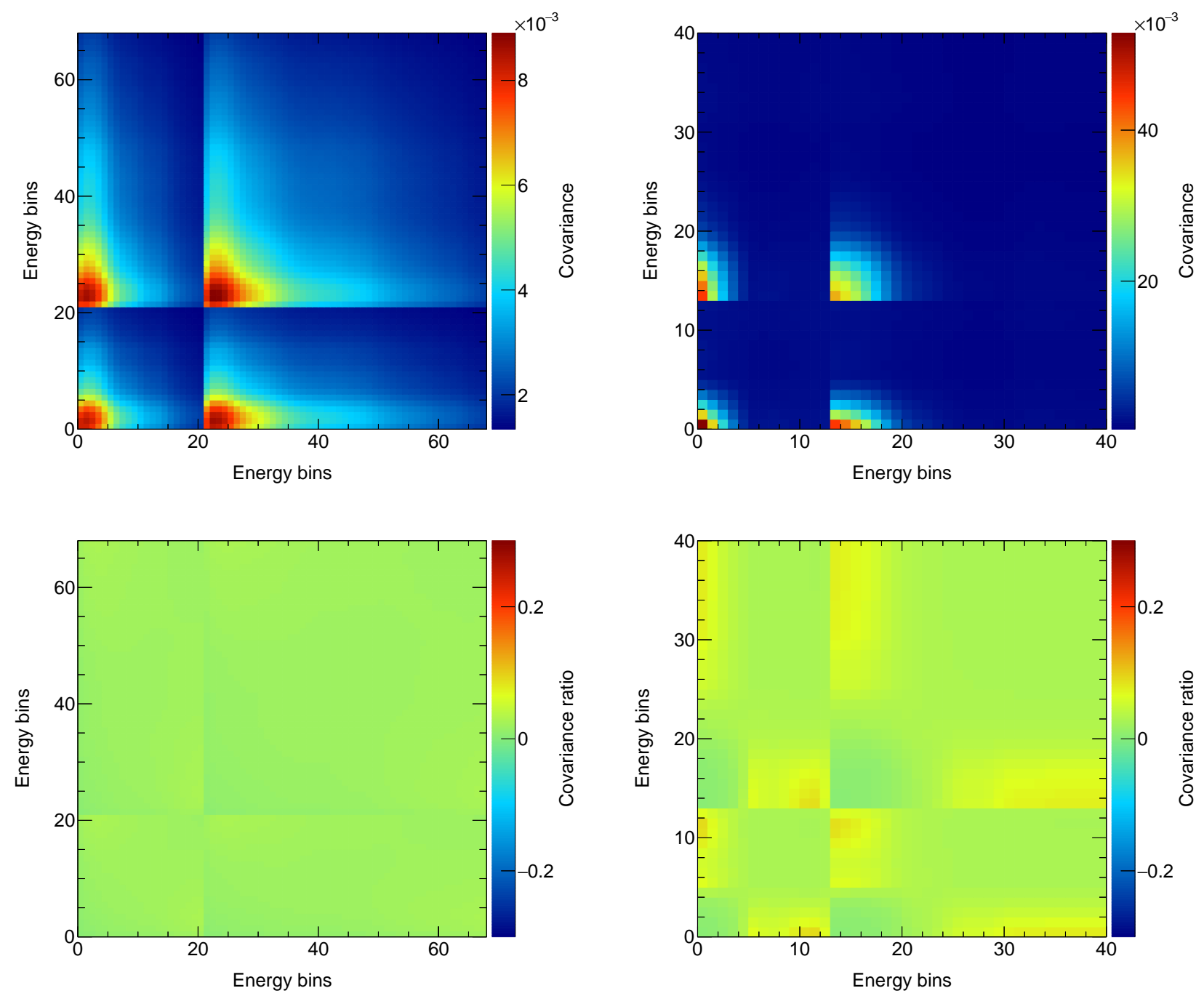

Figure A.9: The original matrices from Fig. A.8 (top row) and the fractional difference of the original and reconstructed matrices for the SVD+QR (bottom row) decomposition obtained from 10000 fluctuated spectra. Unlike in Fig. A.8, the matrices are shown in terms of energy bins rather than reconstructed energy. The left (right) column shows the CC (NC) matrix. 


\section{Appendix B}

\section{A Study of the Asimov Sensitivity Coverage}

This appendix discusses the difference observed at high $\Delta m_{41}^{2}$ values between the Asimov sensitivity coverage and the median of the systematically and statistically fluctuated sensitivity coverages for the MINOS/MINOS $+3+1$ sterile analysis using the two-detector framework [145]. A similar feature is observed in Fig. 6.23 for the MINOS/MINOS+ LED analysis using the twodetector framework, where the difference is most noticeable at high $m_{0}$ values. While the median of a set of fluctuated spectra coincides with the Asimov spectrum, it is not clear that the median of the fluctuated sensitivity coverages should coincide with the Asimov sensitivity coverage, even though this might be the intuitively expected outcome. Studies of this feature suggest that it is not the result of conceptual or coding errors in the various steps of the $3+1$ sterile (LED) analysis, but rather a consequence of the asymmetric nature of the $3+1$ sterile (LED) neutrino oscillations w.r.t. the three-flavor scenario and the observation that the MINOS/MINOS+ systematic uncertainty does not agree well with the expected shape and normalization of sterile neutrino oscillations for high values of $\Delta m_{41}^{2}\left(m_{0}\right)$. It is therefore deemed more representative to compare the data coverage to the median of a set of fluctuated sensitivity coverages rather than to the Asimov sensitivity coverage. 


\section{B.1 Introduction}

While the median of a set of fluctuated spectra coincides with the Asimov spectrum, it is not clear that the median of the fluctuated sensitivity coverages should coincide with the Asimov sensitivity coverage, even though this might be the intuitively expected outcome. The difference is most significant in the region of the $\left(\sin ^{2}\left(\theta_{24}\right), \Delta m_{41}^{2}\right)$ plane where $\Delta m_{41}^{2} \gtrsim 1 \mathrm{eV}^{2}$, as shown in the left panel of Fig. B.1, for which the Near Detector (ND) Charged Current (CC) sample is the dominant contributor to the sensitivity coverage, as can be inferred from Fig. B.2.
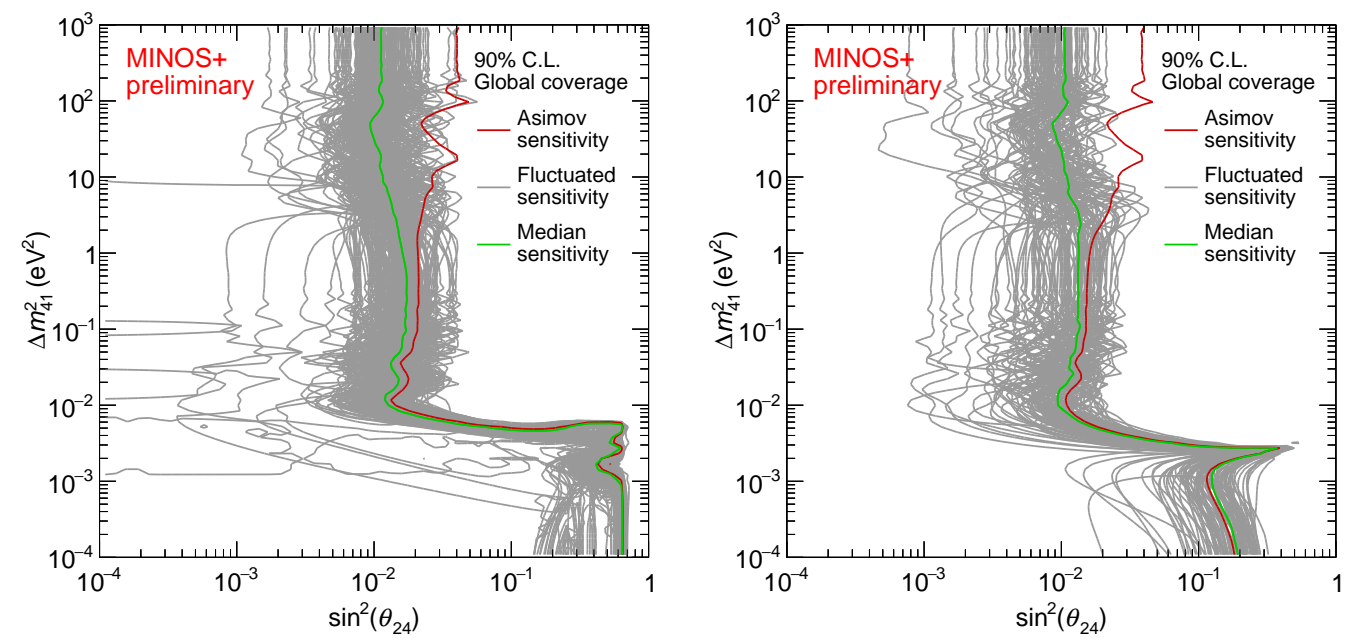

Figure B.1: The Asimov and median fluctuated global 90\% C.L. sensitivity coverages for the two-detector fit method, with 200 fluctuated sensitivity coverages calculated for the full analysis framework (left) and 100 fluctuated sensitivity coverages generated for the case where the atmospheric parameters $\Delta m_{23}^{2}$ and $\theta_{23}$ are fixed to their global fit values (both when generating the fluctuated spectra and in the fit) and with $\theta_{34}$ fixed to 0 (right), as is assumed for the purpose of this study. 

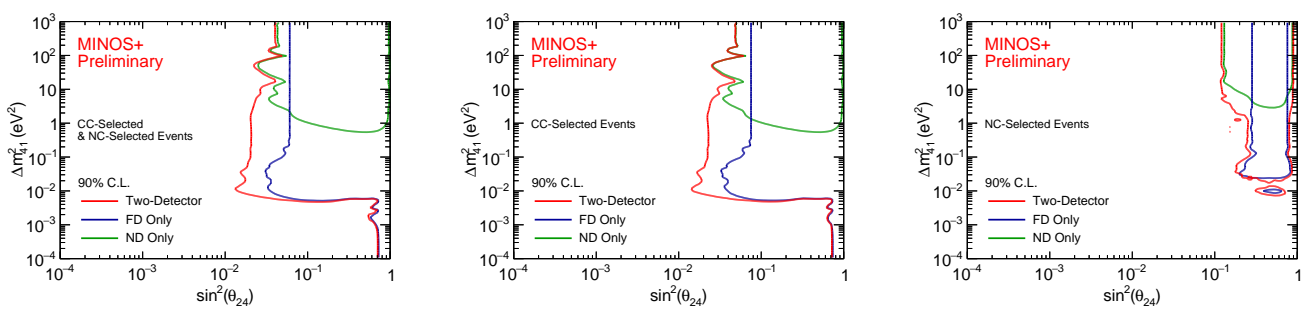

Figure B.2: The global 90\% C.L. Asimov sensitivity coverages for the twodetector fit method compared with the Near Detector and Far Detector independent contributions. Sensitivity coverages are shown for the joint CC and NC-selected samples (left), for the isolated CC-selected sample (middle), and for the isolated NC-selected sample (right). These figures are taken from Ref. [146].

The Asimov spectrum of a sample is defined as the three-flavor simulated data, represented as the neutrino event count versus reconstructed neutrino energy in a binned $0-40 \mathrm{GeV}$ range. It is obtained by multiplying the MINOS/MINOS+ Monte Carlo (MC) prediction without neutrino oscillations for that sample with the appropriate three-flavor oscillation probabilities. The Asimov sensitivity coverage is the sensitivity coverage obtained from comparing the $3+1$ sterile model predictions with the Asimov spectrum using a covariance matrix-based $\chi^{2}$ test statistic. The covariance matrix contains both the systematic and statistical uncertainties of the $3+1$ sterile model prediction and is Gaussian by construction. The systematic uncertainty covariance matrices for the $\mathrm{CC}$ sample that are used in this study are shown in Fig. B.3. The global 90\% C.L Asimov sensitivity coverage corresponds to the $\Delta \chi^{2}=\chi^{2}-\chi_{\min }^{2}=4.61$ (for 2 degrees of freedom) contour in the $\Delta \chi^{2}$ plane spanned by $\sin ^{2}\left(\theta_{24}\right)$ and $\Delta m_{41}^{2}$. 
In the context of this study, the fluctuated three-flavor spectra of a sample are obtained by applying fluctuations to the Asimov spectrum that consist of multivariate Gaussian fluctuations using a covariance matrix containing the three-flavor systematic uncertainties and/or statistical Poisson fluctuations. The fluctuated sensitivity coverages are the sensitivity coverages obtained from comparing the $3+1$ sterile model predictions with the fluctuated spectra using the same covariance matrix-based $\chi^{2}$ test statistic as mentioned above. The global 90\% C.L. fluctuated sensitivity coverages are obtained in the same way as for the Asimov sensitivity.
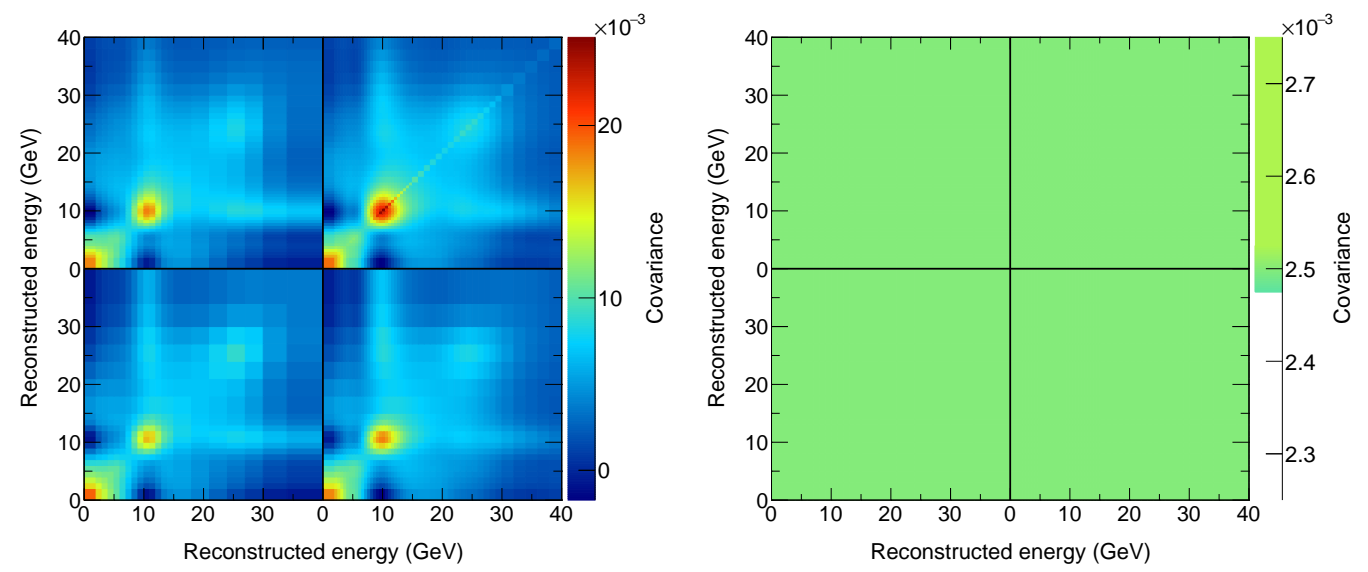

Figure B.3: The CC covariance matrices used for this study, for the full analysis systematic uncertainties (left) and for a simple fully correlated $5 \%$ normalization uncertainty (right). The bottom left and top right quadrants correspond to the Far and Near Detector systematic uncertainties, respectively. The other quadrants contain the correlations between both detectors.

The median fluctuated sensitivity coverage is obtained from the $\Delta \chi^{2}$ planes of all the fluctuated sensitivity coverages by constructing a median $\Delta \chi^{2}$ plane. This is done by ordering within each bin of the $\left(\sin ^{2}\left(\theta_{24}\right), \Delta m_{41}^{2}\right)$ 
plane all the corresponding fluctuated $\Delta \chi^{2}$ values and assigning their median value to that bin in the median $\Delta \chi^{2}$ plane. The $90 \%$ C.L median fluctuated sensitivity coverage then corresponds to the $\Delta \chi^{2}=4.61$ contour in the median $\Delta \chi^{2}$ plane. It should be noted that the median fluctuated sensitivity coverage is not obtained from a fit and as such does not in general correspond to an actual fluctuated three-flavor spectrum.

In what follows several scenarios involving different samples and systematic and statistical uncertainties are presented to understand the observed difference between the Asimov sensitivity coverage and the median of the fluctuated sensitivity coverages. It should be noted that this study does not attempt to give an analytical explanation for this feature. For the purpose of this study a few changes are made w.r.t. the full analysis framework:

\section{- The atmospheric parameters are fixed to their global three-} flavor fit values: $\Delta m_{32}^{2}=2.524 \times 10^{-3} \mathrm{eV}^{2}$ and $\sin ^{2}\left(\theta_{23}\right)=0.44$. In the full $3+1$ sterile analysis framework the atmospheric parameters are allowed to vary in the fitting procedure. Furthermore, to generate a fluctuated spectrum in the full $3+1$ sterile analysis framework the values of the atmospheric parameters assumed for the simulated data are drawn from Gaussian distributions centered around the mean values quoted above to remove a possible bias coming from choosing a specific set of atmospheric parameters when comparing the fluctuated sensitivity coverages to the data coverage. This study requires the generation of many fluctuated sensitivity coverages and fixing the atmospheric parameters 
significantly decreases the computation time. Figure B.4 shows that fixing the atmospheric parameters in the fitting procedure does not affect the Asimov sensitivity coverage for $\Delta m_{41}^{2} \gtrsim 3 \mathrm{eV}^{2}$, the region of most interest for this study, because the there are no three-flavor oscillations along the ND baseline. For $\Delta m_{41}^{2} \lesssim 3 \mathrm{eV}^{2}$, fixing the atmospheric parameters does have a significant effect on the Asimov sensitivity coverage due to the more dominant contribution of the Far Detector (FD) CC sample as $\Delta m_{41}^{2}$ gets smaller (see Fig. B.2).

- The sterile mixing angle $\theta_{34}$ is fixed to 0 . In the full analysis framework this parameter is allowed to vary in the fitting procedure.

Fixing $\theta_{34}$ in addition to the atmospheric parameters means that there are no parameters being fitted at a fixed point in the $\left(\sin ^{2}\left(\theta_{24}\right), \Delta m_{41}^{2}\right)$ plane, thereby significantly reducing the computation time and furthermore avoiding the need for the MINUIT fitting software package in this study (except for the Feldman-Cousins results shown in Fig. B.8). However, it should be noted that the original analysis software is used for this study to allow a direct connection between the observations from this study and the full $3+1$ sterile analysis results. A comparison of the left and right panels in Fig. B.1 shows that these assumptions do not significantly change the observed difference between the Asimov and median fluctuated sensitivity coverages. 

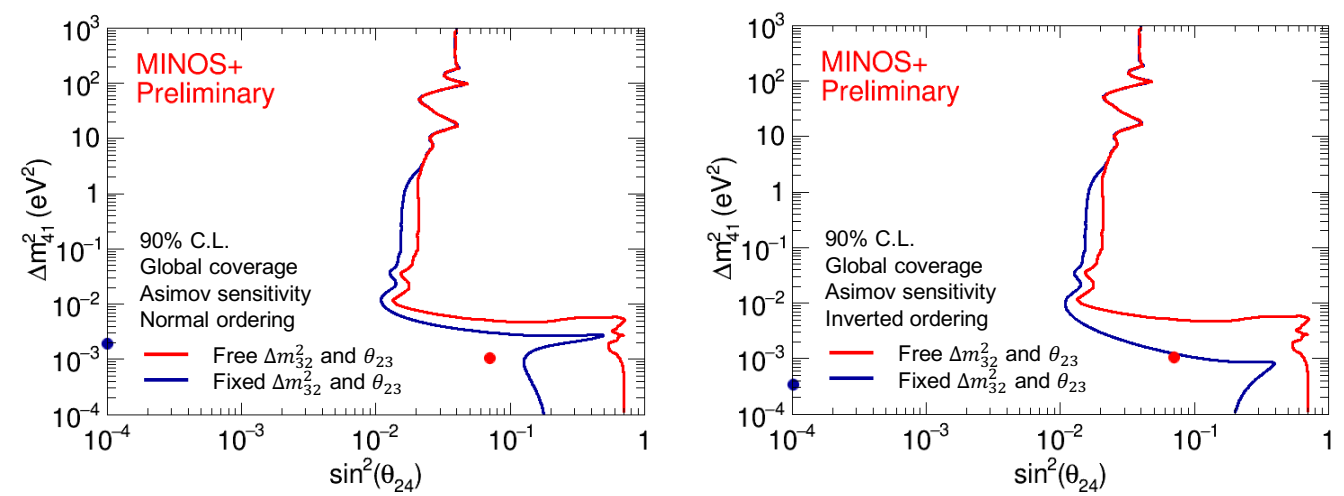

Figure B.4: The effect of fixing the atmospheric parameters $\Delta m_{32}^{2}$ and $\theta_{23}$ in the MINOS/MINOS+ two-detector $3+1$ sterile fit on the global $90 \%$ C.L. Asimov sensitivity coverage, assuming normal ordering (left) and inverted ordering (right). These figures are taken from Ref. [147].

\section{B.2 An ND CC Sample Study}

Because the observed difference between the median fluctuated sensitivity and Asimov sensitivity coverages is most significant in the high $\Delta m_{41}^{2}$ region, a study employing only the ND CC sample is done in this section. This has the added advantage that the ND CC sample is not affected by three-flavor oscillations, such that non-zero values of $\Delta m_{41}^{2}$ and $\sin ^{2}\left(\theta_{24}\right)$ are the only cause for a decrease in neutrino event counts.

\section{B.2.1 Observations}

The top left panel of Fig. B.5 shows the effect on the Asimov (red) and 100 fluctuated sensitivity coverages (gray) of using the full systematic (see the left panel of Fig. B.3) and statistical uncertainties for both the generation of the fluctuated three-flavor spectra and as uncertainty in the $3+1$ 
sterile model predictions when calculating the $\chi^{2}$ values. The median of the fluctuated sensitivity coverages (green) is significantly more constraining than the Asimov sensitivity coverage for $\Delta m_{41}^{2} \gtrsim 1 \mathrm{eV}^{2}$, as is the case in the full two-detector case with free $\theta_{34}$ and atmospheric parameters shown in the left panel of Fig. B.1. The middle left panel shows the same scenario but with all correlations turned off. This is done by setting the off-diagonal elements in the covariance matrix, used for generating the fluctuations and for the $\chi^{2}$ calculation, to zero. As such, only the statistical and diagonal systematic uncertainties are taken into account. Three main observations can be made for the top left two panels:

- the difference between the Asimov and median sensitivity coverages only becomes significant for $\Delta m_{41}^{2}$ values above a few $\mathrm{eV}^{2}$,

- the difference is reduced when turning off correlations, and

- the sensitivity coverages are more constraining when correlations are reduced.

All three observations are confirmed when gradually turning off the correlations from their full value to zero, as shown in Fig. B.6.

It should be noted that the fractional statistical uncertainties in the ND CC sample are very small given the large number of events within each bin, ranging from $\sim 75000$ to $\sim 1.4$ million, and have no significant impact on the results shown in the top and middle left panels. This can also be inferred 
from the bottom left panel of Fig. B.5, where only statistical uncertainties are used to generate the fluctuated spectra and in the $\chi^{2}$ calculations, leading to an increase in coverage of about two orders of magnitude in $\sin ^{2}\left(\theta_{24}\right)$ for $\Delta m_{41}^{2} \gtrsim 1 \mathrm{eV}^{2}$. The good agreement between the Asimov and median sensitivity coverages when using only statistical uncertainties is not surprising, giving statistical fluctuations are uncorrelated and for high event counts behave in a Gaussian manner.

Figure B.7 shows the 90\% C.L. sensitivity coverages (left column) and their corresponding simulated data spectra (right column) for the three same scenarios as in the left column of Fig. B.5.

The panels in the right column of Fig. B.5 show the same three scenarios as the panels on the left for the case where the ND CC spectrum is normalized to the total number of FD events, leading to event counts ranging from 18 to 380 (it should be noted the ND CC spectrum has more than twice the number of bins than the FD CC spectrum). Besides the expected decrease in constraining power due to the lower number of events for all three scenarios, the observations made for the top and middle left panels are still valid. It is also observed that the difference between the Asimov and median sensitivity coverages increases for the statistical uncertainties only case in the bottom right panel w.r.t. to the bottom left panel.

Figure B.8 shows the $\Delta \chi^{2}$ profiles at $\Delta m_{41}^{2}=500 \mathrm{eV}^{2}$ (middle row) and $\Delta m_{41}^{2}=5 \mathrm{eV}^{2}$ (bottom row) for the scenarios of the bottom left panel (left column) and top left panel (right column) in Fig. B.5. These scenarios 
are shown again in the top row of Figure B.8 but with the $\sin ^{2}\left(\theta_{24}\right)$ range extended to $10^{-6}$. The middle and bottom row panels demonstrate how the global $90 \%$ C.L. at $\Delta m_{41}^{2}=500$ and $5 \mathrm{eV}^{2}$ ), shown in the top row panels, can be constructed given the $\Delta \chi^{2}$ profile. The crossings of the $\Delta \chi_{\text {Global }}^{2}=4.61$ line (cyan) with the $\Delta \chi^{2}$ profiles (gray or red) give the global $90 \%$ C.L. values of $\sin ^{2}\left(\theta_{24}\right)$ for the respective $\Delta m_{41}^{2}$ value. Similarly, the crossings of the $\Delta \chi_{\mathrm{FC}}^{2}$ line (magenta) with the $\Delta \chi^{2}$ profiles give the Feldman-Cousins $90 \%$ C.L. values of $\sin ^{2}\left(\theta_{24}\right)$ for the respective $\Delta m_{41}^{2}$ value (it should be noted that the top row panels only show the global $90 \%$ C.L. coverages). From the steep nature of the $\Delta \chi^{2}$ profiles it is apparent that using a Feldman-Cousins interpretation of the $\Delta \chi^{2}$ plane does not change much to the distribution of the $90 \%$ C.L. fluctuated sensitivity coverages around the Asimov sensitivity coverage. In particular, it does not significantly change the difference between the median and Asimov sensitivity coverages at $\Delta m_{41}^{2}=500 \mathrm{eV}^{2}$ for the full analysis systematic and statistical fluctuations case shown in the middle right panel.

\section{B.2.2 Discussion}

A fluctuated sensitivity coverage falling to the right (left) of the Asimov sensitivity coverage at a given value of $\Delta m_{41}^{2}$, i.e. excluding less (more) of the $3+1$ sterile model parameter space than the Asimov sensitivity coverage, corresponds to a simulated data spectrum that looks more (less) like a sterile signal than the Asimov simulated data. 
Both the shape and normalization of the spectrum play a role, with the normalization being special since the effect of non-zero $3+1$ sterile model parameters in any energy bin of the spectrum is to decrease the event count w.r.t. the Asimov event count. Hence, the $3+1$ sterile model, through its oscillation probabilities, introduces an asymmetry in the simulated model predictions that is not present in the distribution of fluctuated simulated data spectra (obtained through the multivariate Gaussian fluctuation procedure) around the Asimov data spectrum. The $3+1$ sterile model event count predictions are always equal to or below the simulated three-flavor event count prediction (Asimov data), while the fluctuated data event counts are distributed around the Asimov event count in a Gaussian manner.

The high $\Delta m_{41}^{2}$ region of the $3+1$ sterile model parameter space plays a special role because, for $\theta_{24} \lesssim 0.3$ or $\sin ^{2}\left(\theta_{24}\right) \lesssim 0.1$, the sterile signals in both the MINOS ND and FD are approximately given by a downward normalization shift only, without any significant shape effects occurring, as is shown in Fig. B.9 and Fig. B.10.

As such, the observations made in Section B.2.1 suggest that the full analysis systematic uncertainty does not agree well with the expected shape and normalization of sterile neutrino oscillations for high values of $\Delta m_{41}^{2}$. This is demonstrated with three fluctuated spectrum examples for the ND CC sample in the top row of Fig. B.7. Comparing the top right and middle right panels in Fig. B.7, it is observed that turning off correlations in the full analysis covariance matrix removes the shape effects in the fluctuated spectra. However, 
rather than looking like an overall normalization offset w.r.t. the Asimov spectrum, they look like random fluctuations (with magnitudes dictated by the systematic uncertainties, as is demonstrated for three examples in the middle row of Fig. B.7) around the Asimov spectrum, thereby reducing but not completely removing the difference between the Asimov and median sensitivity coverages.

To test this hypothesis, a second study using a fully correlated normalization uncertainty is presented in the following section. 


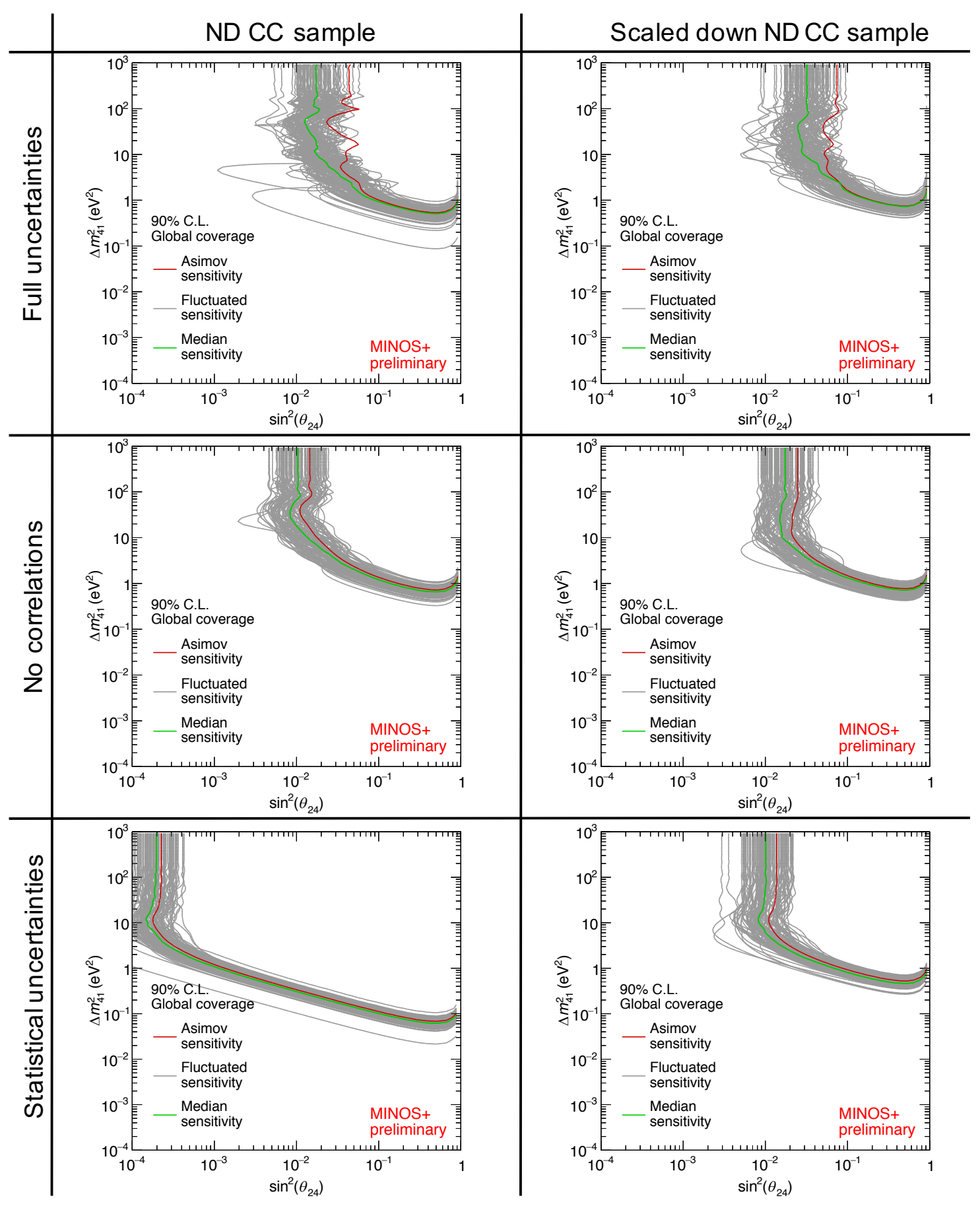

Figure B.5: An ND CC sample study. The effect on the Asimov and fluctuated global 90\% C.L. sensitivity coverages for the ND CC sample when using, both for creating the fluctuated simulated data spectra and calculating the $\chi^{2}$ values, the full analysis systematic and statistical uncertainties (top row), the full analysis systematic and statistical uncertainties with correlations set to zero (middle row), and the statistical uncertainties only (bottom row). This is done for the full ND CC sample (left column) and for a scaled down sample normalized to the total number of FD events (right column). 


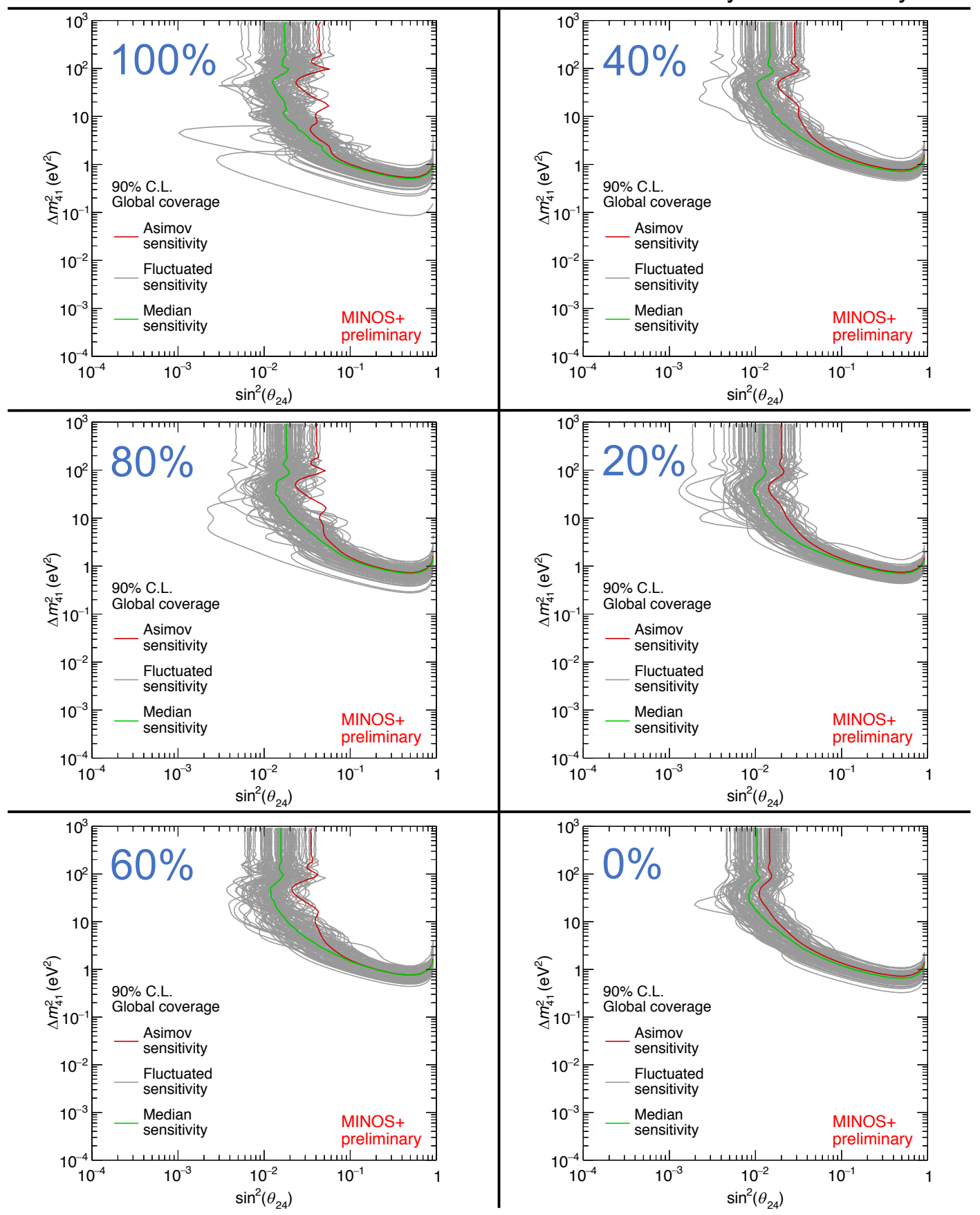

Figure B.6: An ND CC sample study. The effect on the Asimov and fluctuated global $90 \%$ C.L. sensitivity coverages for the ND CC sample when using, both for creating the fluctuated simulated data spectra and calculating the $\chi^{2}$ values, the full analysis systematic and statistical uncertainties for different fractions of the full correlations. 


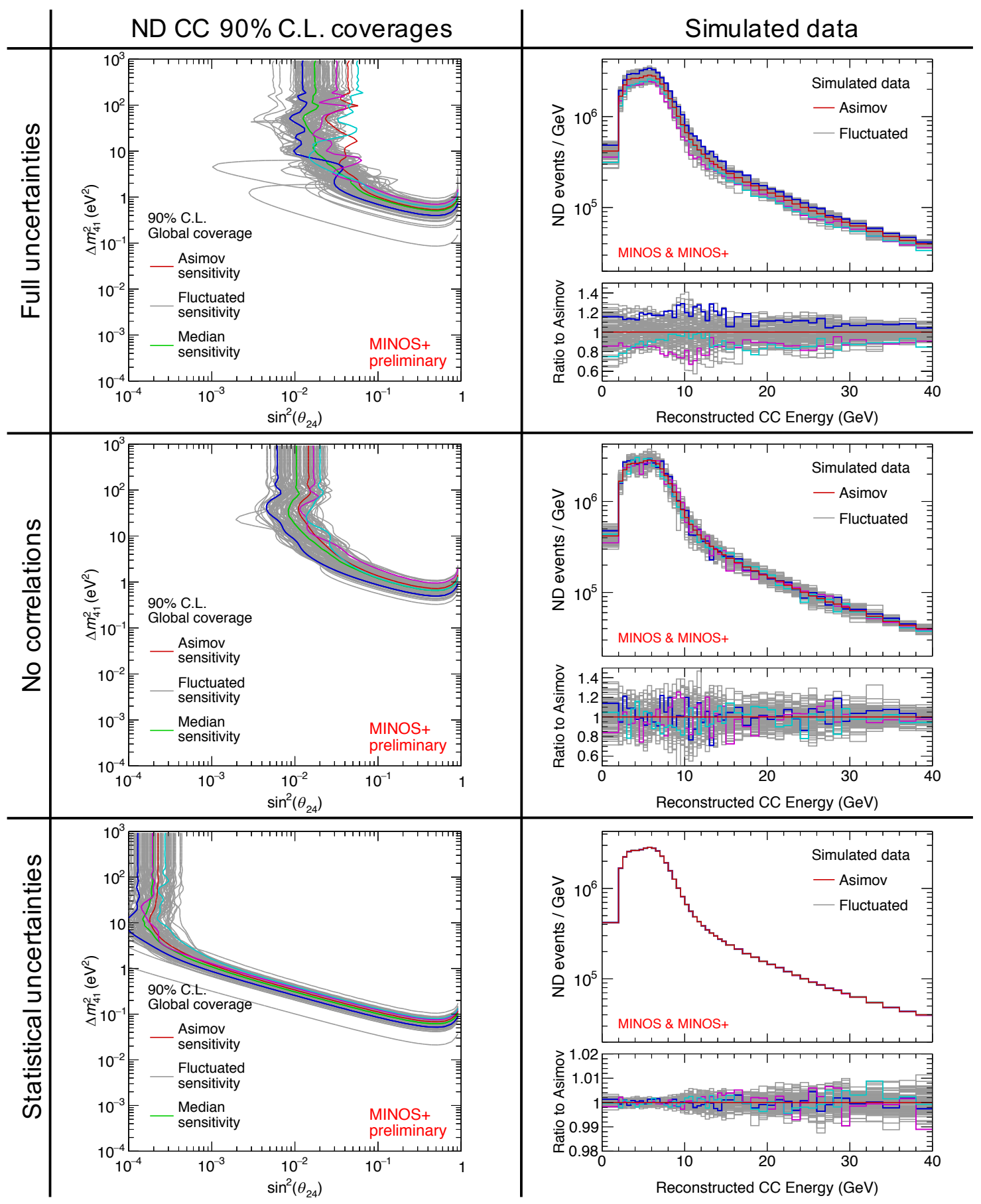

Figure B.7: An ND CC sample study. A selection of simulated data spectra highlighted in color (right column) and their corresponding global 90\% C.L. sensitivity coverages (left column) for the ND CC sample when using, both for creating the fluctuated simulated data spectra and calculating the $\chi^{2}$ values, the full analysis systematic and statistical uncertainties (top row), the full analysis systematic and statistical uncertainties with correlations set to zero (middle row), and the statistical uncertainties only (bottom row). The left column panels are the same as the left column panels in Fig. B.5. 


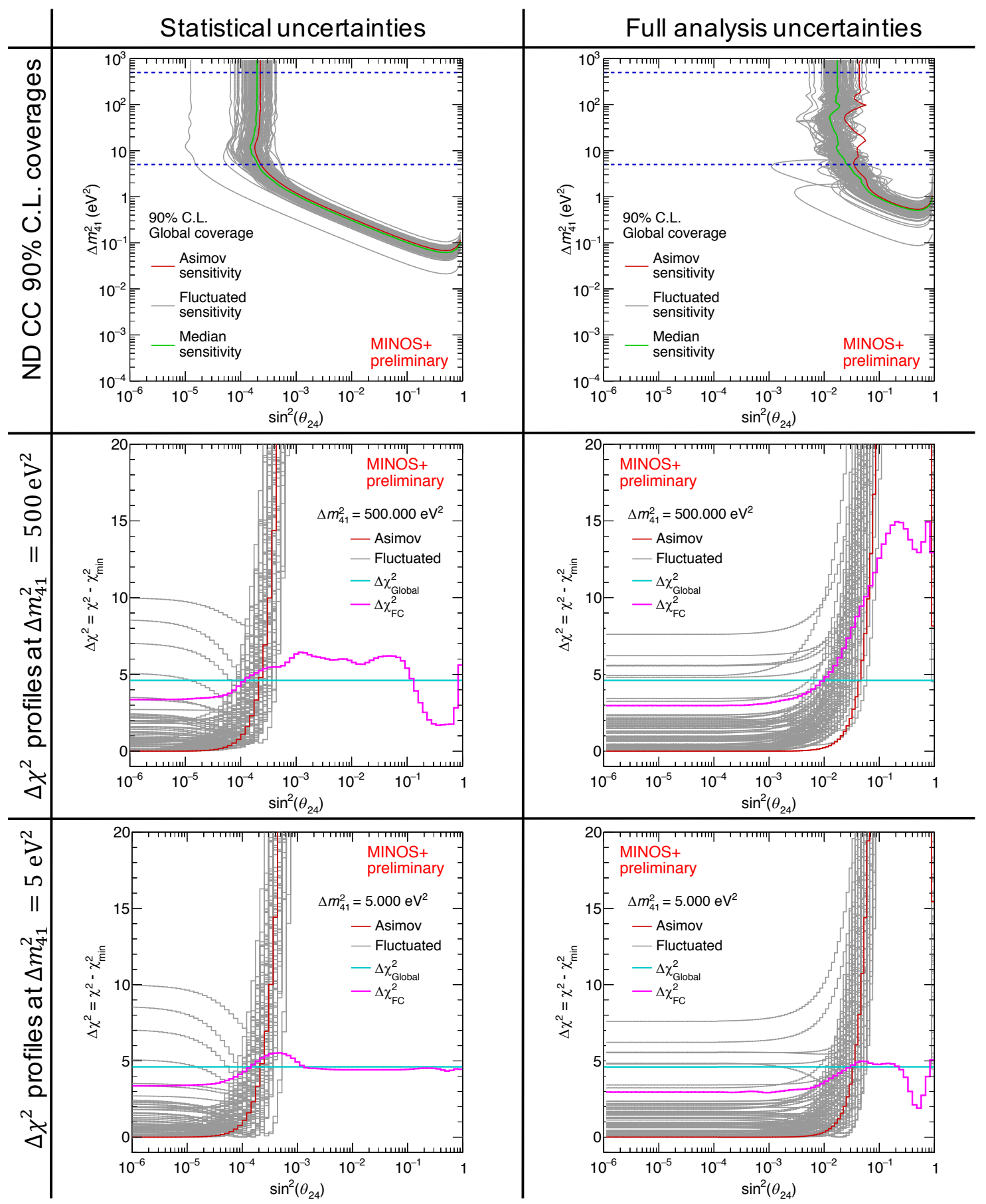

Figure B.8: An ND CC sample study. The $\Delta \chi^{2}$ profiles at $\Delta m_{41}^{2}=500 \mathrm{eV}^{2}$ (middle row) and $\Delta m_{41}^{2}=5 \mathrm{eV}^{2}$ (bottom row) for the ND CC sample when using, both for creating the fluctuated simulated data spectra and calculating the $\chi^{2}$ values, the statistical uncertainties only (left column) and the full analysis systematic and statistical uncertainties (right column). The crossings of the $\Delta \chi_{\text {Global }}^{2}=4.61$ lines (cyan) with the $\Delta \chi^{2}$ profiles give the global $90 \%$ C.L. values of $\sin ^{2}\left(\theta_{24}\right)$ for the respective $\Delta m_{41}^{2}$ value (top row). Similarly, the crossings with the $\Delta \chi_{\mathrm{FC}}^{2}$ lines (magenta) give the Feldman-Cousins $90 \%$ C.L. values of $\sin ^{2}\left(\theta_{24}\right)$. 


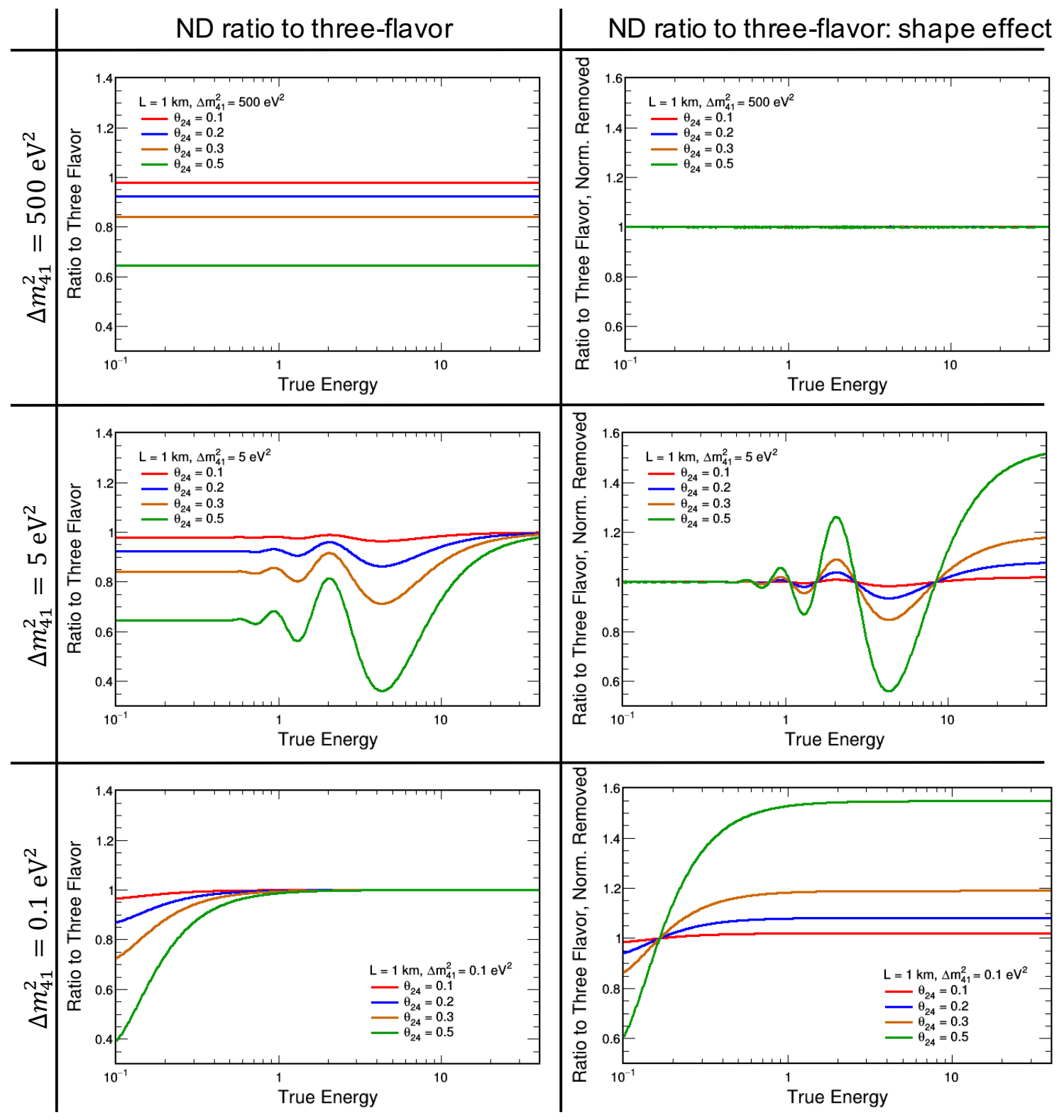

Figure B.9: The ratio of the four-flavor and three-flavor $v_{\mu}$ survival probabilities at the MINOS Near Detector (assuming an oscillation distance of $1 \mathrm{~km}$ ) at $\Delta m_{41}^{2}=500 \mathrm{eV}^{2}$ (top left), $5 \mathrm{eV}^{2}$ (middle left) and $0.1 \mathrm{eV}^{2}$ (bottom left) for different values of $\theta_{24}$. The right column shows the ratio of the four-flavor and three-flavor $v_{\mu}$ survival probabilities divided by $1-\sin ^{2}\left(2 \theta_{24}\right)$ to remove the normalization shift induced by rapid sterile oscillations. These figures are taken from Ref. [146]. 


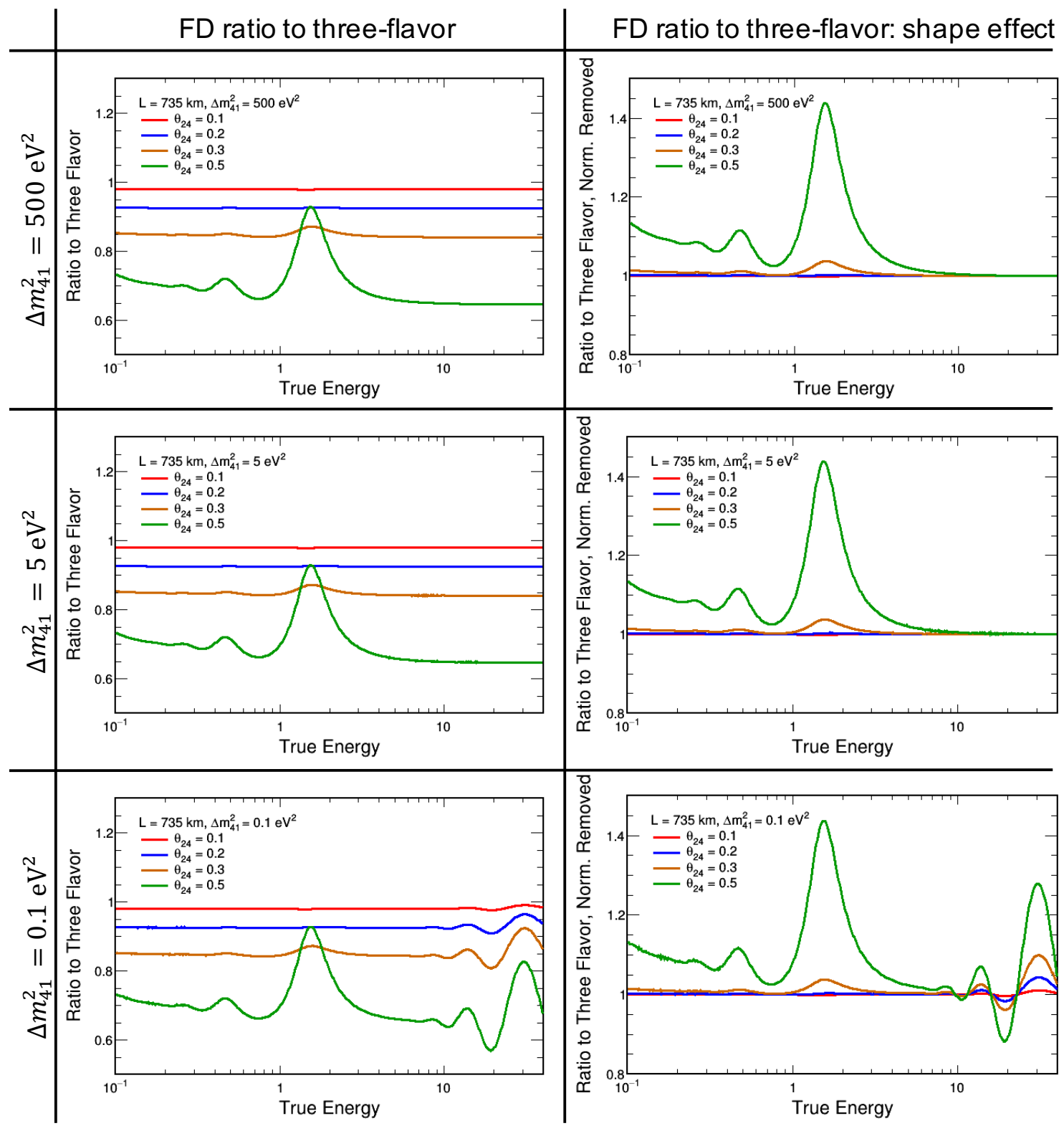

Figure B.10: The ratio of the four-flavor and three-flavor $v_{\mu}$ survival probabilities at the MINOS Far Detector (assuming an oscillation distance of $1 \mathrm{~km}$ ) at $\Delta m_{41}^{2}=500 \mathrm{eV}^{2}$ (top left), $5 \mathrm{eV}^{2}$ (middle left) and $0.1 \mathrm{eV}^{2}$ (bottom left) for different values of $\theta_{24}$. The right column shows the ratio of the four-flavor and three-flavor $v_{\mu}$ survival probabilities divided by $1-\sin ^{2}\left(2 \theta_{24}\right)$ to remove the normalization shift induced by rapid sterile oscillations. These figures are taken from Ref. [146]. 


\section{B.3 A Fully Correlated 5\% Normalization Uncertainty Study}

To simplify matters, a covariance matrix representing a fully correlated $5 \%$ normalization uncertainty is used in the study described in this section. Such a covariance matrix has both diagonal and off-diagonal elements equal to $0.05 \times 0.05=0.0025$, as is shown in the right panel of Fig. B.3. Employing the multivariate Gaussian fluctuation procedure with this matrix is equivalent to drawing one random number from a Gaussian distribution with mean 1 and standard deviation 1 and multiplying it with the $5 \%$ uncertainty to obtain the shift for the complete spectrum. Hence, the ratio of the resulting fluctuated spectrum is energy independent, as can be seen for the ND CC sample, the FD CC sample, and the combined ND and FD CC samples in the right column of Fig. B.11. From Fig. B.9 and Fig. B.10 it follows that such normalization offsets obtained in this study can mimic sterile neutrino oscillations at high $\Delta m_{41}^{2}$ values.

\section{B.3.1 Mimicking Sterile Neutrino Oscillation Signals for High $\Delta m_{41}^{2}$ Values}

Figure B.11 shows the $90 \%$ C.L. sensitivity coverages (left column) and their corresponding simulated data spectra (right column) for fluctuations using only the fully correlated $5 \%$ normalization uncertainty. The fluctuated spectra are compared to the $3+1$ sterile model predictions using the full analysis systematic uncertainty matrix (left panel of Fig. B.3) as the covariance matrix in the $\chi^{2}$ calculation. The aim is to see how the same fitting frame- 
work as in Section B.2, responds to simulated data that matches sterile signals in the high $\Delta m_{41}^{2}$ region. For all three scenarios presented in Fig. B.11, the median sensitivity coverage agrees well with the Asimov sensitivity coverage. A selection of fluctuated spectra and their corresponding sensitivity coverages are highlighted in color for illustrative purposes and discussed below.

The yellow ND CC fluctuated three-flavor spectrum in the top right panel of Fig. B.11 corresponds to the most extreme upward normalization shift of the 100 fluctuated cases. Its corresponding 90\% C.L. sensitivity coverage is more constraining than the $90 \%$ C.L. Asimov sensitivity coverage, as is shown in the top left panel. The same is observed for any of the fluctuated spectra that correspond to an upward normalization shift. Sterile oscillations at high $\Delta m_{41}^{2}$ values can only result in downward shifts w.r.t. the Asimov prediction, such that upward shifts are excluded more strongly in the $\left(\sin ^{2}\left(\theta_{24}\right), \Delta m_{41}^{2}\right)$ plane than the Asimov spectrum. The cyan ND CC fluctuated three-flavor spectrum in the top right panel corresponds to a $4 \%$ downward shift w.r.t. the Asimov spectrum and leads to less exclusion for $\Delta m_{41}^{2} \gtrsim 10 \mathrm{eV}^{2}$ and slightly more exclusion for $\Delta m_{41}^{2} \lesssim 10 \mathrm{eV}^{2}$ w.r.t. to the Asimov sensitivity coverage. The flip to less exclusion above $10 \mathrm{eV}^{2}$ occurs because at lower $\Delta m_{41}^{2}$ values a downward normalization shift cannot mimic sterile oscillations anymore, as is shown in the middle and bottom rows of Fig. B.9. An increasingly downward normalization shift leads to less exclusion for $\Delta m_{41}^{2} \gtrsim 10 \mathrm{eV}^{2}$ and $\sin ^{2}\left(\theta_{24}\right) \gtrsim 0.06$, and more exclusion for $\sin ^{2}\left(\theta_{24}\right) \lesssim 0.06$, thereby narrowing the region of $3+1$ sterile model predictions in the $\left(\sin ^{2}\left(\theta_{24}\right), \Delta m_{41}^{2}\right)$ plane that 
could mimic such a downward shift, as can be seen from the orange, magenta, and blue cases. Similar observations can be made for the FD CC sample and the combined ND and FD CC sample cases shown in the middle and bottom rows of Fig. B.11.

These tests show that the fitting procedure produces the expected results in the high $\Delta m_{41}^{2}$ region of the parameter space when confronted with simulated data that mimics sterile signals.

\section{B.3.2 Turning Off Correlations in the Fluctuation Process}

The effect of gradually turning down correlations in the normalization matrix used to generate the ND CC fluctuated spectra shown in Fig. B.11 is shown in Fig. B.12 and Fig. B.13. In this case, turning down correlations gradually removes the normalization offset and introduces random noise, making the fluctuated spectra look less like a sterile signal in the high $\Delta m_{41}^{2}$ region and thereby causing an increasing difference between the median of the fluctuated sensitivity coverages and the Asimov sensitivity coverage.

Figure B.13 shows for three different fluctuated spectra with a constant normalization shift w.r.t. the Asimov spectrum, how turning off the correlations in the fluctuation process increases the sensitivity coverage due to an increasing mismatch between the $3+1$ sterile model predictions and the simu-

lated data. The cyan fluctuated spectrum in the top right panel of Fig. B.13 corresponds to a $12 \%$ downward shift w.r.t. to the Asimov spectrum, obtained using the multivariate Gaussian fluctuation procedure with the fully correlated 
$5 \%$ normalization uncertainty matrix. The corresponding sensitivity coverage in the top left panel is closed around the Asimov sensitivity coverage at high values of $\Delta m_{41}^{2}$ given such a strong downward normalization shift can only match a narrow region of $3+1$ sterile model predictions. Turning down the correlations in the normalization matrix to $90 \%$ (orange spectrum) decreases the normalization offset and introduces random noise. This in turn opens the 90\% C.L. sensitivity coverage because such a signal looks less like a sterile neutrino signal at high $\Delta m_{41}^{2}$ values. Further turning down the correlations pushes the sensitivity coverage to even smaller $\theta_{24}$ values. Similar observations are made for a $5 \%$ downward normalization shift in the middle row of Fig. B.13. Turning down correlations in the fluctuation process for a $10 \%$ upward normalization shift, shown in the bottom row of Fig. B.13, has no big effect on the fluctuated sensitivity coverage at high $\Delta m_{41}^{2}$ values.

Figure B.14 is the equivalent of Fig. B.6 for the case of the normalization uncertainty, with the normalization covariance matrix from the right panel of Fig. B.3 being used both for the generation of the fluctuated three-flavor spectra and as systematic uncertainty for the $3+1$ sterile model predictions in the $\chi^{2}$ calculation. In this case, the gradual removal of the normalization shift in both the fluctuations and predictions by turning down correlations initially rapidly increases the difference between the Asimov and median sensitivity coverages. With decreasing correlations, the normalization offset decreases and the fluctuated spectra turn into random fluctuations around the Asimov spectrum such that the difference between the Asimov and median sensitivity 
coverages decreases again and disappears when correlations are completely turned off.

\section{B.3.3 Turning Off Correlations in the $\chi^{2}$ Calculation}

Finally, Fig. B.15 shows how the sensitivity coverage for a given fluctuated spectrum changes when turning down the correlations in the normalization uncertainty matrix used as systematic uncertainty for the $3+1$ sterile model predictions in the $\chi^{2}$ calculation. The top row panels show that for a fluctuated data spectrum that can mimic sterile oscillations at high $\Delta m_{41}^{2}$ values, the sensitivity coverage closes in on a smaller part of the $\left(\sin ^{2}\left(\theta_{24}\right), \Delta m_{41}^{2}\right)$ plane when correlations are turned down. Turning off correlations in the fitting procedure makes fewer $3+1$ model predictions match with the simulated data. Two other examples are shown in the middle and bottom row of Fig. B.15, for which similar observations are made in the high $\Delta m_{41}^{2}$ region of the parameter space. 


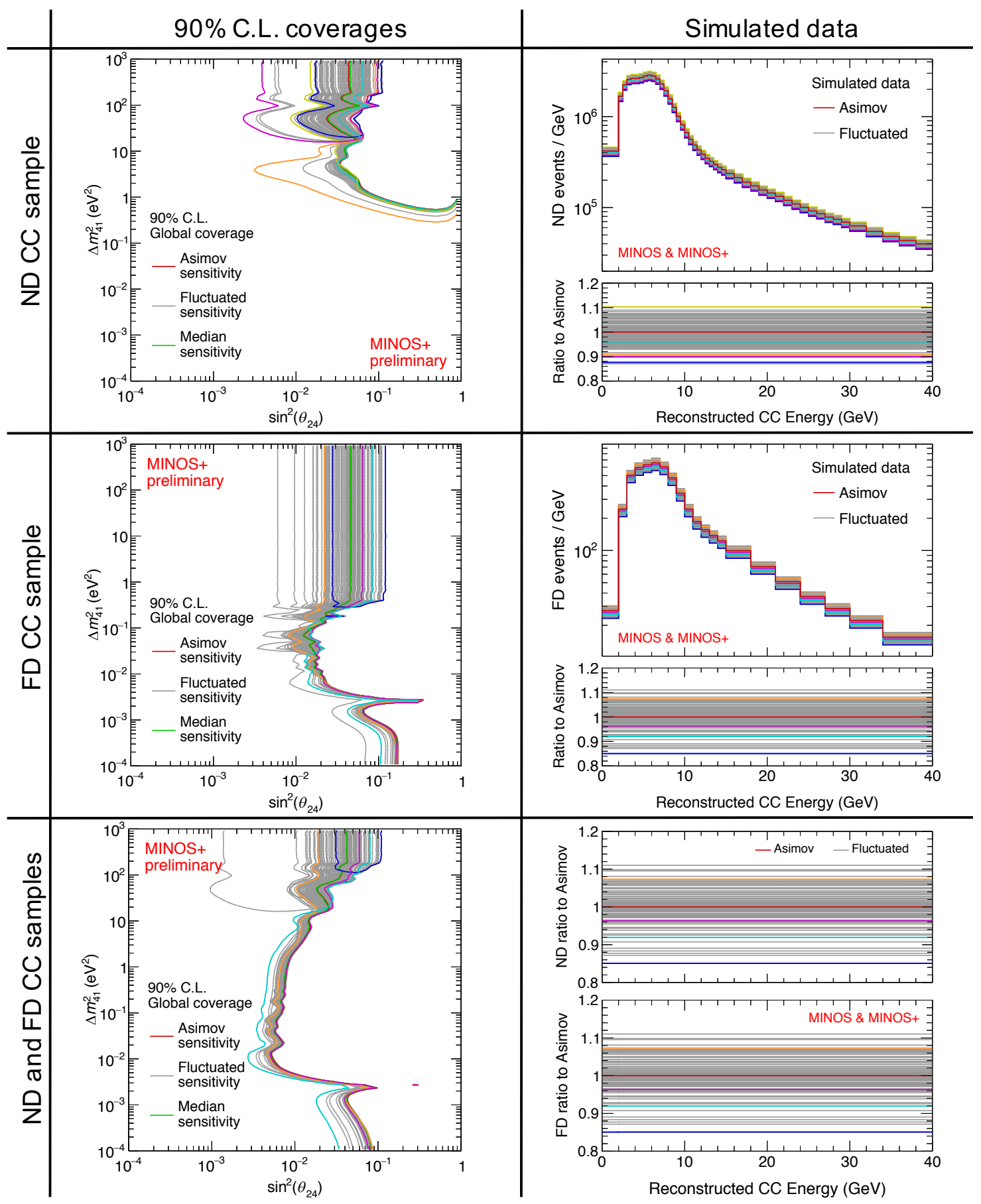

Figure B.11: A fully correlated 5\% normalization uncertainty study. A selection of simulated data spectra highlighted in color (right column) and their corresponding global $90 \%$ C.L. sensitivity coverages (left column) for the ND CC sample (top row), the FD CC sample (middle row), and the ND and FD CC samples (bottom row). The full analysis systematic and statistical uncertainties are used as the uncertainties for the $3+1$ sterile model predictions in the $\chi^{2}$ calculations. 

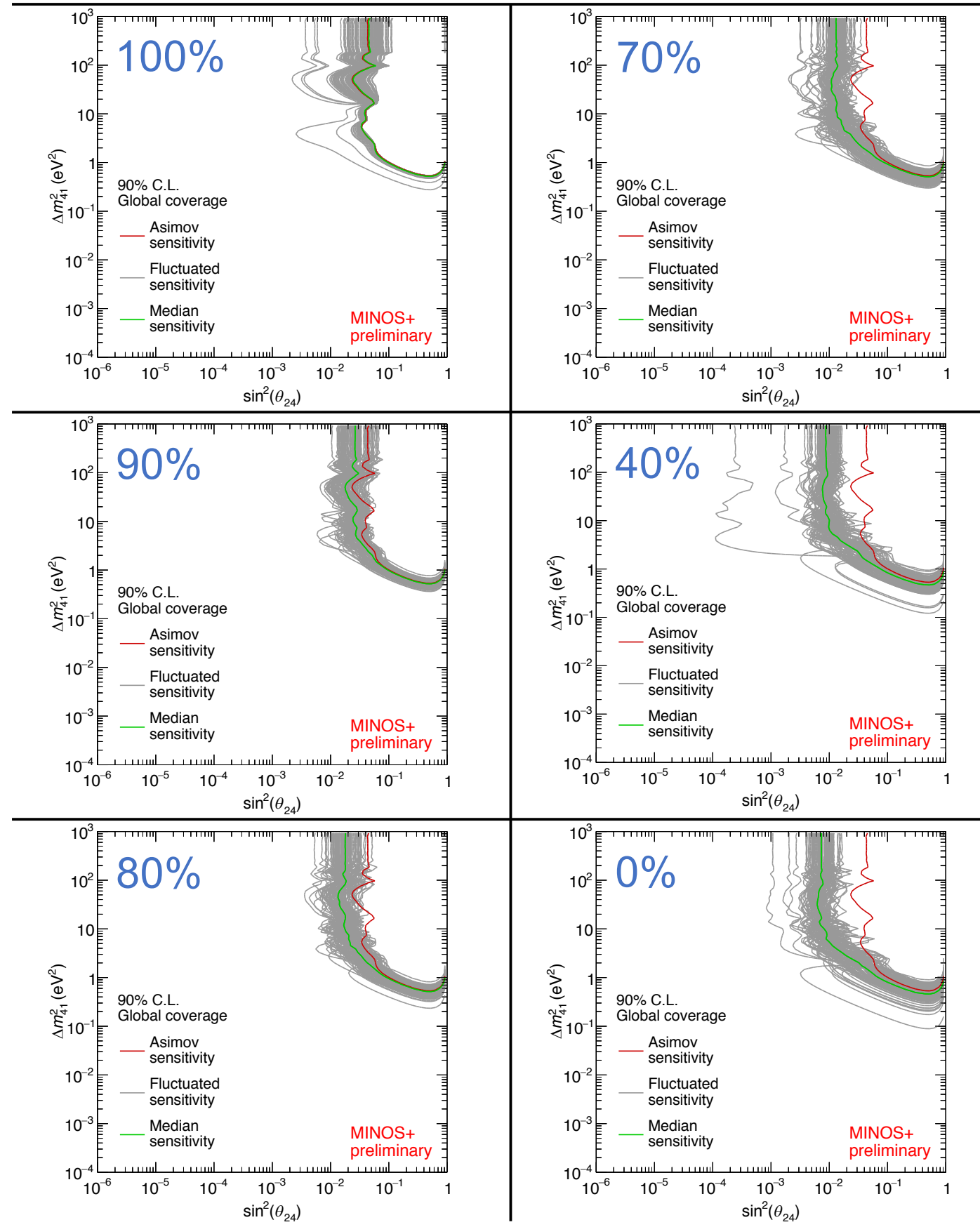

Figure B.12: A fully correlated 5\% normalization uncertainty study. The effect on the Asimov and fluctuated global 90\% C.L. sensitivity coverages for the ND CC sample when using the fully correlated $5 \%$ normalization uncertainties with different fractions of the full correlations for creating the fluctuated simulated data spectra and using the full analysis systematic and statistical uncertainties for calculating the $\chi^{2}$ values. 


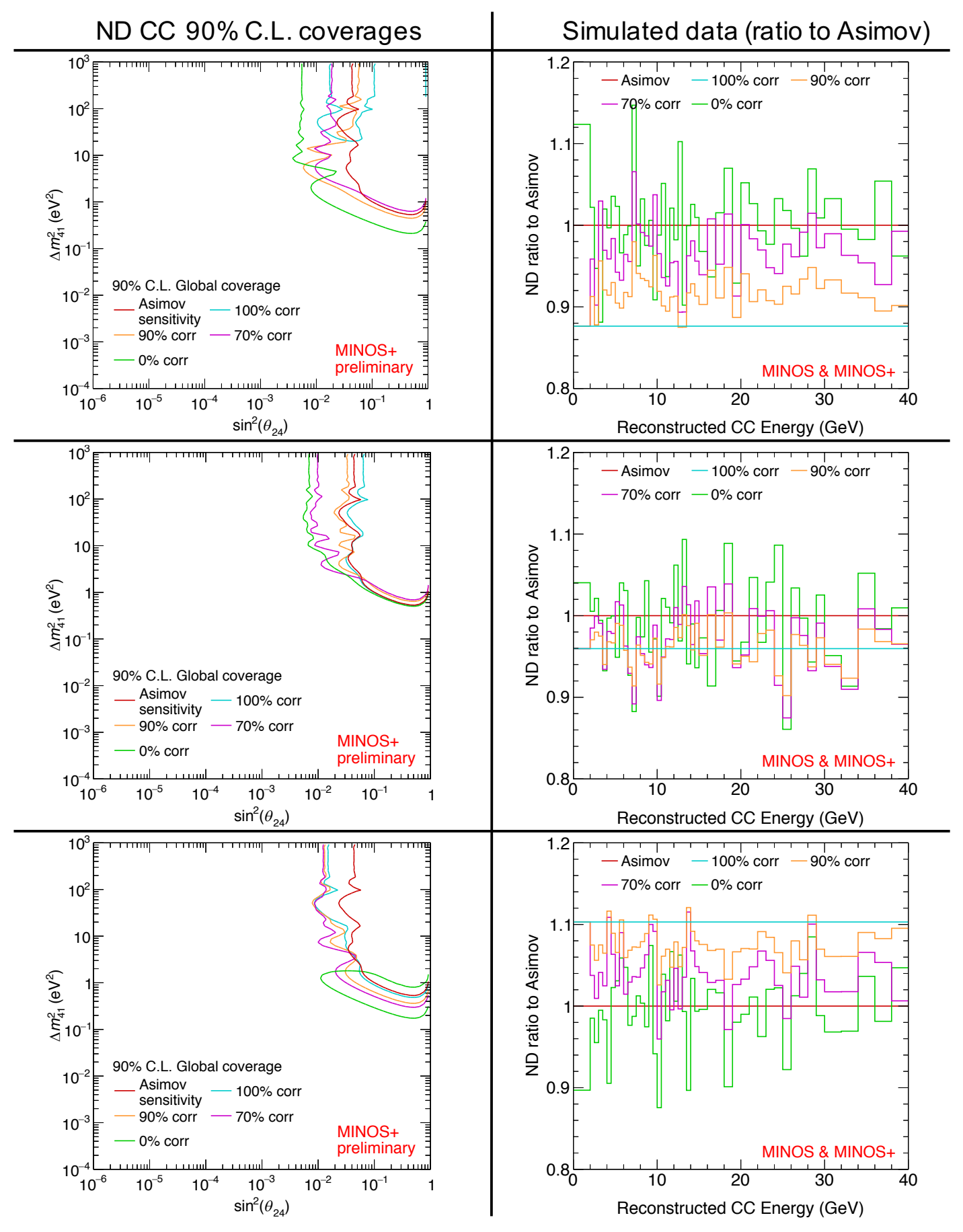

Figure B.13: A fully correlated $5 \%$ normalization uncertainty study. The effect of shape and normalization of the fluctuated spectra on the fluctuated global $90 \%$ C.L. sensitivity coverages for the ND CC sample when using the fully correlated $5 \%$ normalization uncertainties, with different fractions of the full correlations for creating the fluctuated simulated data spectra, and using the full analysis systematic and statistical uncertainties for calculating the $\chi^{2}$ values. 


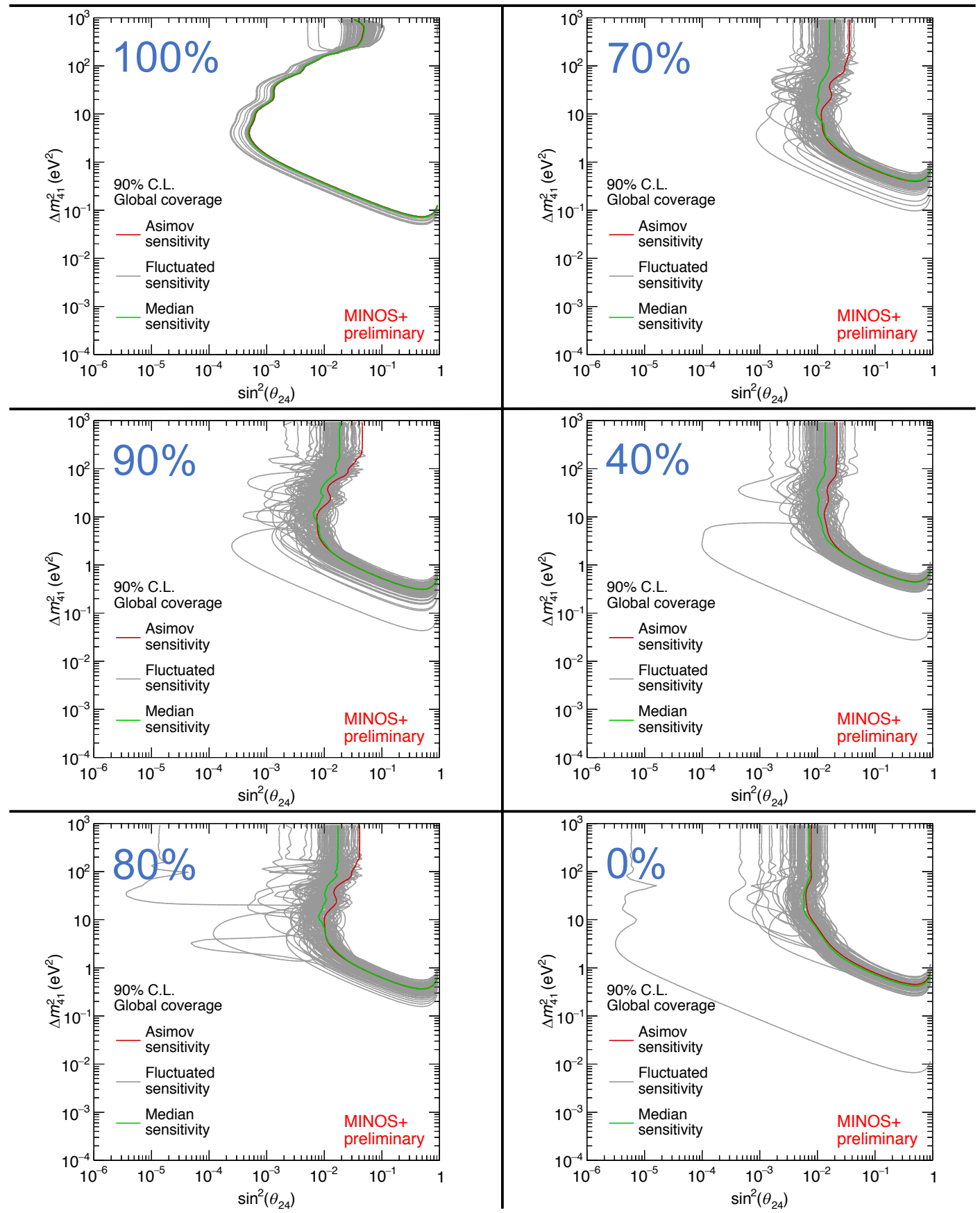

Figure B.14: A fully correlated 5\% normalization uncertainty study. The effect on the Asimov and fluctuated global 90\% C.L. sensitivity coverages for the ND CC sample when using the fully correlated $5 \%$ normalization uncertainties, both for creating the fluctuated simulated data spectra and calculating the $\chi^{2}$ values, for different fractions of the full correlations. 


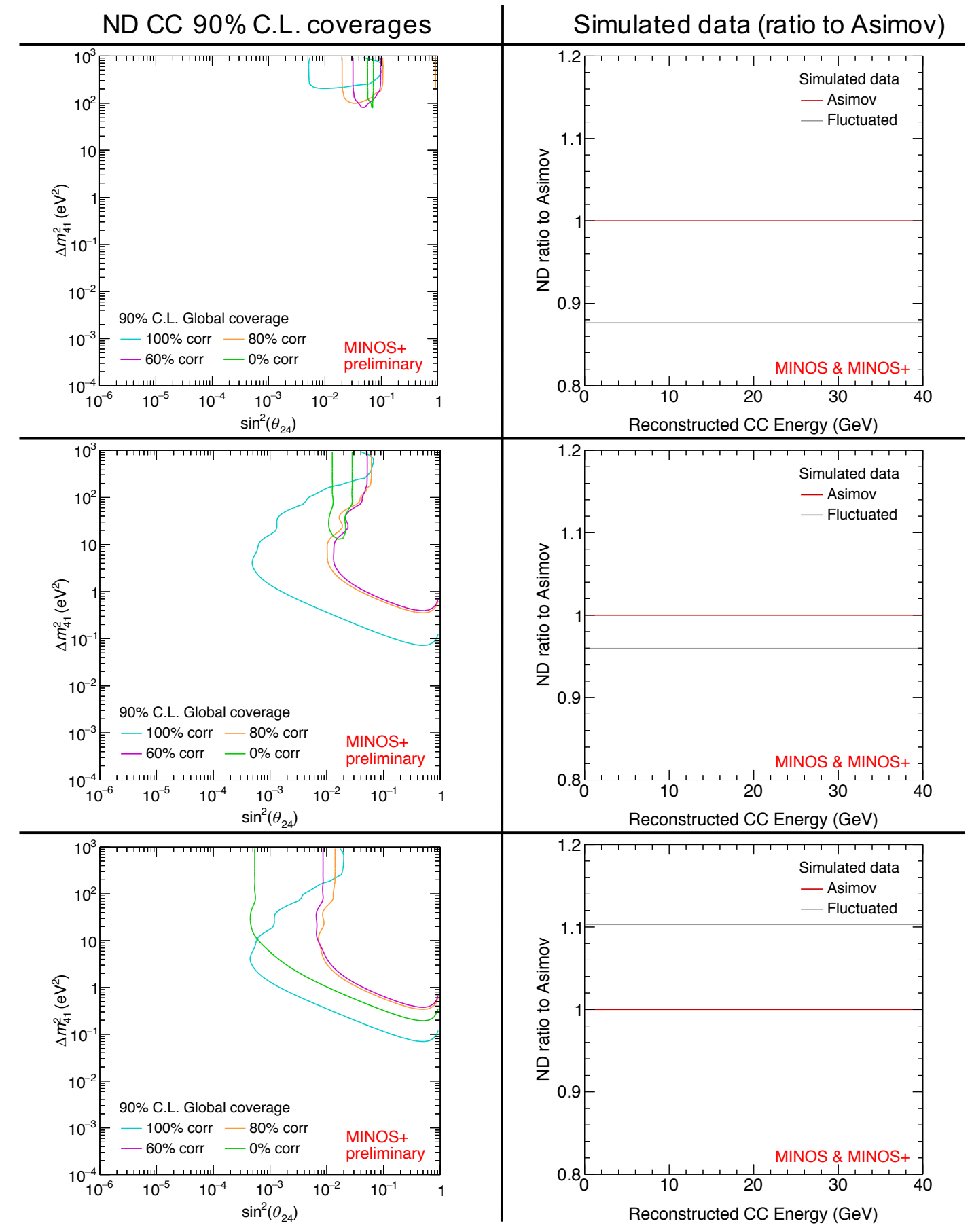

Figure B.15: A fully correlated 5\% normalization uncertainty study. The effect of shape and normalization of the fluctuated spectra on the fluctuated global 90\% C.L. sensitivity coverages for the ND CC sample when using the fully correlated $5 \%$ normalization uncertainties for creating the fluctuated simulated data spectra and using the fully correlated $5 \%$ normalization uncertainties with different fractions of the full correlations for calculating the $\chi^{2}$ values. 


\section{B.4 Conclusions}

Various searches for conceptual and coding errors where conducted for the different steps in the $3+1$ sterile analysis, in particular the generation of the fluctuated simulated data spectra and the calculation of the $\chi^{2}$ test statistic, and no such errors were found. In addition, studies of the behavior of the Asimov and median fluctuated sensitivity coverages in various scenarios, with a focus on the high $\Delta m_{41}^{2}$ region of the $\left(\sin ^{2}\left(\theta_{24}\right), \Delta m_{41}^{2}\right)$ plane, indicate that the difference between them is a consequence of

- the asymmetric nature of the $3+1$ sterile neutrino oscillations w.r.t. the three-flavor scenario, with non-zero sterile parameters only leading to a decrease in the number of observed neutrino events, and

- the nature of the systematic uncertainties used for the MINOS/MINOS+ $3+1$ sterile analysis using the two-detector framework, which are not highly correlated with the expected shape and normalization of sterile neutrino oscillations at high $\Delta m_{41}^{2}$ values.

More specifically, when employing a systematic uncertainty that does mimic sterile neutrino oscillations, an agreement between the Asimov and median fluctuated sensitivity coverages is observed. Given these observations, a comparison of the data coverage to the median of a set of fluctuated sensitivity coverages rather than to the Asimov sensitivity coverage is deemed more representative. 


\section{Bibliography}

[1] O. von Bayer, O. Hahn, and L. Meitner, "Nachweis von $\beta$-Strahlen bei Radium D," Physikalische Zeitschrift, vol. 12, p. 378, 1911.

[2] W. Pauli, "Dear radioactive ladies and gentlemen," Phys.Today, vol. 31N9, p. 27, 1978.

[3] E. Fermi, "Trends to a Theory of beta Radiation. (In Italian)," Nuovo Cim., vol. 11, pp. 1-19, 1934. [,535(1934)].

[4] C. Cowan, F. Reines, F. Harrison, H. Kruse, and A. McGuire, "Detection of the free neutrino: A Confirmation," Science, vol. 124, pp. 103-104, 1956.

[5] G. Danby, J. Gaillard, K. A. Goulianos, L. Lederman, N. B. Mistry, et al., "Observation of High-Energy Neutrino Reactions and the Existence of Two Kinds of Neutrinos," Phys.Rev.Lett., vol. 9, pp. 36-44, 1962.

[6] B. Pontecorvo, "Electron and Muon Neutrinos," Sov.Phys.JETP, vol. 10, pp. 1236-1240, 1960.

[7] K. Kodama et al., "Observation of tau neutrino interactions," Phys.Lett., vol. B504, pp. 218-224, 2001. 
[8] M. L. Perl, G. Abrams, A. Boyarski, M. Breidenbach, D. Briggs, et al., "Evidence for Anomalous Lepton Production in e+ - e- Annihilation," Phys.Rev.Lett., vol. 35, pp. 1489-1492, 1975.

[9] S. Schael et al., "Precision electroweak measurements on the $Z$ resonance," Phys.Rept., vol. 427, pp. 257-454, 2006.

[10] F. Englert and R. Brout, "Broken Symmetry and the Mass of Gauge Vector Mesons," Phys. Rev. Lett., vol. 13, pp. 321-323, 1964. [,157(1964)].

[11] P. W. Higgs, "Broken Symmetries and the Masses of Gauge Bosons," Phys. Rev. Lett., vol. 13, pp. 508-509, 1964. [,160(1964)].

[12] G. S. Guralnik, C. R. Hagen, and T. W. B. Kibble, "Global Conservation Laws and Massless Particles," Phys. Rev. Lett., vol. 13, pp. 585-587, 1964. [,162(1964)].

[13] S. L. Glashow, "Partial Symmetries of Weak Interactions," Nucl. Phys., vol. 22, pp. 579-588, 1961.

[14] S. Weinberg, "A Model of Leptons," Phys. Rev. Lett., vol. 19, pp. 12641266, 1967.

[15] A. Salam, "Weak and Electromagnetic Interactions," Conf. Proc., vol. C680519, pp. 367-377, 1968.

[16] N. Aghanim et al., "Planck intermediate results. XLVI. Reduction of large-scale systematic effects in HFI polarization maps and estimation 
of the reionization optical depth," Astron. Astrophys., vol. 596, p. A107, 2016.

[17] C. Patrignani et al., "Review of Particle Physics," Chin. Phys., vol. C40, no. 10, p. 100001, 2016.

[18] C. Wu, E. Ambler, R. Hayward, D. Hoppes, and R. Hudson, "Experimental Test of Parity Conservation in Beta Decay," Phys.Rev., vol. 105, pp. 1413-1414, 1957.

[19] M. Goldhaber, L. Grodzins, and A. Sunyar, "Helicity of Neutrinos," Phys.Rev., vol. 109, pp. 1015-1017, 1958.

[20] B. Pontecorvo, "Mesonium and anti-mesonium," Sov.Phys.JETP, vol. 6, p. $429,1957$.

[21] B. Pontecorvo, "Inverse beta processes and nonconservation of lepton charge," Sov.Phys.JETP, vol. 7, pp. 172-173, 1958.

[22] Z. Maki, M. Nakagawa, and S. Sakata, "Remarks on the unified model of elementary particles," Prog.Theor.Phys., vol. 28, pp. 870-880, 1962.

[23] L. Wolfenstein, "Neutrino Oscillations in Matter," Phys. Rev., vol. D17, pp. 2369-2374, 1978. [,294(1977)].

[24] S. P. Mikheev and A. Yu. Smirnov, "Resonance Amplification of Oscillations in Matter and Spectroscopy of Solar Neutrinos," Sov. J. Nucl. Phys., vol. 42, pp. 913-917, 1985. [,305(1986)]. 
[25] Y. Fukuda et al., "Evidence for oscillation of atmospheric neutrinos," Phys.Rev.Lett., vol. 81, pp. 1562-1567, 1998.

[26] B. Aharmim et al., "Electron energy spectra, fluxes, and day-night asymmetries of B-8 solar neutrinos from measurements with $\mathrm{NaCl}$ dissolved in the heavy-water detector at the Sudbury Neutrino Observatory," Phys. Rev., vol. C72, p. 055502, 2005.

[27] T. Araki et al., "Measurement of neutrino oscillation with KamLAND: Evidence of spectral distortion," Phys. Rev. Lett., vol. 94, p. 081801, 2005.

[28] M. H. Ahn et al., "Measurement of Neutrino Oscillation by the K2K Experiment," Phys. Rev., vol. D74, p. 072003, 2006.

[29] F. An et al., "Observation of electron-antineutrino disappearance at Daya Bay," Phys.Rev.Lett., vol. 108, p. 171803, 2012.

[30] P. Adamson et al., "Combined analysis of $\nu_{\mu}$ disappearance and $\nu_{\mu} \rightarrow \nu_{e}$ appearance in MINOS using accelerator and atmospheric neutrinos," Phys.Rev.Lett., vol. 112, p. 191801, 2014.

[31] F. Capozzi, E. Di Valentino, E. Lisi, A. Marrone, A. Melchiorri, and A. Palazzo, "Global constraints on absolute neutrino masses and their ordering," Phys. Rev. D, vol. 95, p. 096014, May 2017. 
[32] F. Capozzi, G. Fogli, E. Lisi, A. Marrone, D. Montanino, et al., "Status of three-neutrino oscillation parameters, circa 2013," Phys.Rev., vol. D89, no. 9, p. 093018, 2014.

[33] J. Hewett, H. Weerts, R. Brock, J. Butler, B. Casey, et al., "Fundamental Physics at the Intensity Frontier," 2012.

[34] A. Aguilar-Arevalo et al., "Evidence for neutrino oscillations from the observation of anti-neutrino(electron) appearance in a anti-neutrino(muon) beam," Phys.Rev., vol. D64, p. 112007, 2001.

[35] A. Aguilar-Arevalo et al., "Improved Search for $\bar{\nu}_{\mu} \rightarrow \bar{\nu}_{e}$ Oscillations in the MiniBooNE Experiment," Phys.Rev.Lett., vol. 110, p. 161801, 2013.

[36] M. A. Acero, C. Giunti, and M. Laveder, "The Gallium and reactor neutrinos anomaly," Nucl. Phys. Proc. Suppl., vol. 188, pp. 211-213, 2009.

[37] G. Mention, M. Fechner, T. Lasserre, T. Mueller, D. Lhuillier, et al., "The Reactor Antineutrino Anomaly," Phys.Rev., vol. D83, p. 073006, 2011.

[38] F. P. An et al., "Evolution of the Reactor Antineutrino Flux and Spectrum at Daya Bay," Phys. Rev. Lett., vol. 118, no. 25, p. 251801, 2017.

[39] P. Huber, "NEOS Data and the Origin of the $5 \mathrm{MeV}$ Bump in the Reactor Antineutrino Spectrum," Phys. Rev. Lett., vol. 118, no. 4, p. 042502, 2017. 
[40] Y. Declais, J. Favier, A. Metref, H. Pessard, B. Achkar, et al., "Search for neutrino oscillations at 15-meters, 40-meters, and 95-meters from a nuclear power reactor at Bugey," Nucl.Phys., vol. B434, pp. 503-534, 1995.

[41] A. Romosan et al., "A High statistics search for muon-neutrino (antimuon-neutrino) $\rightarrow$ electron-neutrino (anti-electron-neutrino) oscillations in the small mixing angle regime," Phys.Rev.Lett., vol. 78, pp. 29122915, 1997.

[42] D. Autiero, "Talk presented at the 1998 international conference on high energy physics in vancouver, canada.."

[43] K. Eitel, "talk presented at the 2000 international neutrino conference in sudbury, canada.."

[44] B. Armbruster et al., "Upper limits for neutrino oscillations muon-antineutrino $\rightarrow$ electron-anti-neutrino from muon decay at rest," Phys.Rev., vol. D65, p. 112001, 2002.

[45] J. Huang, Sterile Neutrino Searches in MINOS/MINOS+ Experiment. PhD thesis, U. Texas, Austin (main), 2015.

[46] A. M. Timmons, Search for Sterile Neutrinos with the MINOS LongBaseline Experiment. PhD thesis, Manchester U., 2016. 
[47] P. Adamson et al., "Search for Sterile Neutrinos Mixing with Muon Neutrinos in MINOS," Phys. Rev. Lett., vol. 117, no. 15, p. 151803, 2016.

[48] P. Adamson et al., "Limits on Active to Sterile Neutrino Oscillations from Disappearance Searches in the MINOS, Daya Bay, and Bugey3 Experiments," Phys. Rev. Lett., vol. 117, no. 15, p. 151801, 2016. [Addendum: Phys. Rev. Lett.117,no.20,209901(2016)].

[49] N. Arkani-Hamed, S. Dimopoulos, and G. Dvali, "The Hierarchy problem and new dimensions at a millimeter," Phys.Lett., vol. B429, pp. 263-272, 1998.

[50] L. Randall and R. Sundrum, "A Large mass hierarchy from a small extra dimension," Phys. Rev. Lett., vol. 83, pp. 3370-3373, 1999.

[51] L. Randall and R. Sundrum, "An alternative to compactification," Phys. Rev. Lett., vol. 83, pp. 4690-4693, Dec 1999.

[52] P. Minkowski, " $\mu \rightarrow e \gamma$ at a Rate of One Out of $10^{9}$ Muon Decays?," Phys. Lett., vol. 67B, pp. 421-428, 1977.

[53] M. Gell-Mann, P. Ramond, and R. Slansky, "Complex Spinors and Unified Theories," Conf. Proc., vol. C790927, pp. 315-321, 1979.

[54] O. Sawada and A. Sugamoto, eds., Proceedings: Workshop on the Unified Theories and the Baryon Number in the Universe, (Tsukuba, Japan), Natl.Lab.High Energy Phys., Natl.Lab.High Energy Phys., 1979. 
[55] R. N. Mohapatra and G. Senjanovic, "Neutrino Mass and Spontaneous Parity Violation," Phys. Rev. Lett., vol. 44, p. 912, 1980.

[56] K. R. Dienes, E. Dudas, and T. Gherghetta, "Neutrino oscillations without neutrino masses or heavy mass scales: A Higher dimensional seesaw mechanism," Nucl. Phys., vol. B557, p. 25, 1999.

[57] N. Arkani-Hamed, S. Dimopoulos, G. Dvali, and J. March-Russell, "Neutrino masses from large extra dimensions," Phys.Rev., vol. D65, p. $024032,2002$.

[58] G. R. Dvali and A. Yu. Smirnov, "Probing large extra dimensions with neutrinos," Nucl. Phys., vol. B563, pp. 63-81, 1999.

[59] R. Barbieri, P. Creminelli, and A. Strumia, "Neutrino oscillations from large extra dimensions," Nucl. Phys., vol. B585, pp. 28-44, 2000.

[60] R. N. Mohapatra, S. Nandi, and A. Perez-Lorenzana, "Neutrino masses and oscillations in models with large extra dimensions," Phys. Lett., vol. B466, pp. 115-121, 1999.

[61] R. N. Mohapatra and A. Perez-Lorenzana, "Sterile neutrino as a bulk neutrino," Nucl. Phys., vol. B576, pp. 466-478, 2000.

[62] R. N. Mohapatra and A. Perez-Lorenzana, "Three flavor neutrino oscillations in models with large extra dimensions," Nucl. Phys., vol. B593, pp. 451-470, 2001. 
[63] H. Davoudiasl, P. Langacker, and M. Perelstein, "Constraints on large extra dimensions from neutrino oscillation experiments," Phys.Rev., vol. D65, p. 105015, 2002.

[64] P. Machado, H. Nunokawa, and R. Zukanovich Funchal, "Testing for Large Extra Dimensions with Neutrino Oscillations," Phys.Rev., vol. D84, p. 013003, 2011.

[65] A. Esmaili, O. L. G. Peres, and Z. Tabrizi, "Probing Large Extra Dimensions With IceCube," JCAP, vol. 1412, no. 12, p. 002, 2014.

[66] J. M. Berryman, A. de Gouvêa, K. J. Kelly, O. L. G. Peres, and Z. Tabrizi, "Large extra dimensions at the deep underground neutrino experiment," Phys. Rev. D, vol. 94, p. 033006, Aug 2016.

[67] P. A. N. Machado, H. Nunokawa, F. A. Pereira dos Santos, and R. Z. Funchal, "Bulk neutrinos as an alternative cause of the gallium and reactor anti-neutrino anomalies," Phys. Rev. D, vol. 85, p. 073012, Apr 2012.

[68] M. Carena, Y.-Y. Li, C. S. Machado, P. A. N. Machado, and C. E. M. Wagner, "Neutrinos in large extra dimensions and short-baseline $\nu_{e}$ appearance," Phys. Rev. D, vol. 96, p. 095014, Nov 2017.

[69] S. Hannestad and G. G. Raffelt, "Supernova and neutron star limits on large extra dimensions reexamined," Phys.Rev., vol. D67, p. 125008, 2003. 
[70] L. J. Hall and D. Tucker-Smith, "Cosmological constraints on theories with large extra dimensions," Phys.Rev., vol. D60, p. 085008, 1999.

[71] C. Hanhart, J. A. Pons, D. R. Phillips, and S. Reddy, "The Likelihood of GODs' existence: Improving the SN1987a constraint on the size of large compact dimensions," Phys.Lett., vol. B509, pp. 1-9, 2001.

[72] E. Adelberger, J. Gundlach, B. Heckel, S. Hoedl, and S. Schlamminger, "Torsion balance experiments: A low-energy frontier of particle physics," Prog.Part.Nucl.Phys., vol. 62, pp. 102-134, 2009.

[73] "Aleph, delphi, 13, opal collaborations and the lep exotica working group, lep exotica wg 2004-03, aleph 2004-007, delphi 2004-033 conf 708, 13 note 2798, opal technical note tn743.." http://lepexotica.web.cern.ch/ LEPEXOTICA/, 2004.

[74] J. Abdallah et al., "Search for one large extra dimension with the DELPHI detector at LEP," Eur.Phys.J., vol. C60, pp. 17-23, 2009.

[75] F. P. An et al., "Independent measurement of the neutrino mixing angle $\theta_{13}$ via neutron capture on hydrogen at Daya Bay," Phys. Rev., vol. D90, no. 7, p. $071101,2014$.

[76] J. H. Choi et al., "Observation of Energy and Baseline Dependent Reactor Antineutrino Disappearance in the RENO Experiment," Phys. Rev. Lett., vol. 116, no. 21, p. 211801, 2016. 
[77] Y. Abe et al., "Improved measurements of the neutrino mixing angle $\theta_{13}$ with the Double Chooz detector," JHEP, vol. 10, p. 086, 2014. [Erratum: JHEP02,074(2015)].

[78] M. A. Kordosky, "Hadronic interactions in the MINOS detectors," 2004.

[79] J. S. Mitchell, "Measuring $\nu_{\mu}$ Disappearance with the MINOS Experiment," 2011.

[80] P. Harrison, D. Perkins, and W. Scott, "Tri-bimaximal mixing and the neutrino oscillation data," Physics Letters B, vol. 530, no. 1, pp. 167 173, 2002.

[81] D. Michael et al., "The Magnetized steel and scintillator calorimeters of the MINOS experiment," Nucl.Instrum.Meth., vol. A596, pp. 190-228, 2008.

[82] P. Adamson et al., "The NuMI Neutrino Beam," Nucl. Instrum. Meth., vol. A806, pp. 279-306, 2016.

[83] G. Tzanankos et al., "MINOS+: a Proposal to FNAL to run MINOS with the medium energy NuMI beam," 2011.

[84] P. Adamson et al., "First direct observation of muon antineutrino disappearance," Phys.Rev.Lett., vol. 107, p. 021801, 2011.

[85] P. Adamson et al., "Search for sterile neutrinos in MINOS and MINOS+ using a two-detector fit," Submitted to: Phys. Rev. Lett., 2017. 
[86] P. Adamson et al., "Constraints on Large Extra Dimensions from the MINOS Experiment," Phys. Rev., vol. D94, no. 11, p. 111101, 2016.

[87] P. Adamson et al., "Search for flavor-changing nonstandard neutrino interactions using $\nu_{e}$ appearance in MINOS," Phys. Rev., vol. D95, no. 1, p. $012005,2017$.

[88] P. Adamson et al., "Search for flavor-changing non-standard neutrino interactions by MINOS," Phys. Rev., vol. D88, no. 7, p. 072011, 2013.

[89] P. Adamson et al., "Measurement of single $\pi^{0}$ production by coherent neutral-current $\nu$-Fe interactions in the MINOS Near Detector," Phys. Rev., vol. D94, no. 7, p. 072006, 2016.

[90] P. Adamson et al., "Study of quasielastic scattering using chargedcurrent $\nu_{\mu}$-iron interactions in the MINOS near detector," Phys. Rev., vol. D91, no. 1, p. 012005, 2015.

[91] L. Aliaga et al., "Neutrino Flux Predictions for the NuMI Beam," Phys. Rev., vol. D94, no. 9, p. 092005, 2016. [Addendum: Phys. Rev.D95,no.3,039903(2017)].

[92] A. Pla-Dalmau, "Extruded plastic scintillator for the MINOS calorimeters," Frascati Phys. Ser., vol. 21, pp. 513-522, 2001. [,513(2001)].

[93] N. Tagg et al., "Performance of Hamamatsu 64-anode photomultipliers for use with wavelength-shifting optical fibres," Nucl. Instrum. Meth., vol. A539, pp. 668-678, 2005. 
[94] K. Lang et al., "Characterization of 1600 Hamamatsu 16-anode photomultipliers for the MINOS Far detector," Nucl. Instrum. Meth., vol. A545, pp. 852-871, 2005.

[95] T. Cundiff et al., "The MINOS near detector front end electronics," IEEE Trans. Nucl. Sci., vol. 53, pp. 1347-1355, 2006.

[96] J. Oliver, N. Felt, G. Feldman, A. Lebedev, and R. Lee, "Design and performance of the readout system of the MINOS Far Detector," IEEE Trans. Nucl. Sci., vol. 51, pp. 2193-2195, 2004.

[97] J. J. Evans, "Measuring Antineutrino Oscillations with the MINOS Experiment," 2008.

[98] T. J. Carroll, "Muon Neutrino Disappearance Measurement at MINOS+," J. Phys. Conf. Ser., vol. 888, no. 1, p. 012161, 2017.

[99] P. Adamson et al., "The MINOS calibration detector," Nucl. Instrum. Meth., vol. A556, pp. 119-133, 2006.

[100] P. Adamson et al., "On the linearity of the MINOS light-injection calibration system," Nucl. Instrum. Meth., vol. A521, pp. 361-366, 2004.

[101] A. J. Perch, Three-flavour neutrino oscillations with MINOS and CHIPS. PhD thesis, University Coll. London, 2017-01-17.

[102] A. Blake, "Far detector timing calibration, minos document 11967," April 2014. 
[103] J. J. Hartnell, Measurement of the Calorimetric Energy Scale in MINOS. PhD thesis, Oxford U., 2005.

[104] M. Campanella, A. Ferrari, P. R. Sala, and S. Vanini, "First Calorimeter Simulation with the FLUGG Prototype," 1999.

[105] M. Campanella, A. Ferrari, P. R. Sala, and S. Vanini, "Reusing Code from FLUKA and GEANT4 Geometry," 1998.

[106] S. Agostinelli et al., "GEANT4: A Simulation toolkit," Nucl. Instrum. Meth., vol. A506, pp. 250-303, 2003.

[107] F. Ballarini et al., "Nuclear models in FLUKA: Present capabilities, open problems and future improvements," AIP Conf. Proc., vol. 769, pp. 1197-1202, 2005. [,1197(2004)].

[108] Z. Pavlovic, Observation of Disappearance of Muon Neutrinos in the NuMI Beam. PhD thesis, Texas U., 2008.

[109] A. Schreckenberger, "Data-monte carlo agreement, minos internal document 10689," July 2014.

[110] M. Dorman, "Beam fit position paper, minos internal document 7146," April 2010.

[111] H. Gallagher, "The NEUGEN neutrino event generator," Nucl.Phys.Proc.Suppl., vol. 112, pp. 188-194, 2002. 
[112] R. Hatcher and S. Kasahara, "The (attempt at a semi-) definitive guide to minos geometry, minos internal document 7828," May 2010.

[113] C. Zeitnitz and T. A. Gabriel, "The GEANT - CALOR interface and benchmark calculations of ZEUS test calorimeters," Nucl. Instrum. Meth., vol. A349, pp. 106-111, 1994.

[114] R. Fruhwirth, "Application of Kalman filtering to track and vertex fitting," Nucl. Instrum. Meth., vol. A262, pp. 444-450, 1987.

[115] J. S. Marshall, "A study of muon neutrino disappearance with the MINOS detectors and the NuMI neutrino beam," 2008.

[116] C. Smith, "Ss event builder algorithm, minos internal document 2821," March 2007.

[117] C. J. Backhouse, "Measuring neutrino oscillation parameters using $\nu_{\mu}$ disappearance in MINOS," 2011.

[118] L. Whitehead, "Summary of the minos+ intensity effect, minos internal document 11358," February 2016.

[119] S. De Rijck, "Minos+ fhc mc linearity study, minos internal document 11480," May 2016.

[120] A. Aurisano, M. Kordoski, J. Todd, and L. Whitehead, "Blessed plots for the 2016 fhc sterile analysis, minos internal document 11583," June 2016. 
[121] G. M. Tinti, "Sterile neutrino oscillations in MINOS and hadron production in pC collisions," 2010.

[122] R. Ospanov, "A measurement of muon neutrino disappearance with the MINOS detectors and NuMI beam," 2008.

[123] J. S. Ratchford, "Identifying Muons for Neutrino Oscillation and Cross Section Experiments,"

[124] D. Michael et al., "Observation of muon neutrino disappearance with the MINOS detectors and the NuMI neutrino beam," Phys.Rev.Lett., vol. 97, p. 191801, 2006.

[125] P. Adamson et al., "An improved measurement of muon antineutrino disappearance in MINOS," Phys.Rev.Lett., vol. 108, p. 191801, 2012.

[126] S. V. Cao, Study of antineutrino oscillations using accelerator and atmospheric data in MINOS. PhD thesis, Texas U., 2014.

[127] F. James and M. Roos, "Minuit - a system for function minimization and analysis of the parameter errors and correlations," Computer Physics Communications, vol. 10, no. 6, pp. 343 - 367, 1975.

[128] J. Coelho, J. Evans, and A. Sousa, "The problem with dynamic scaling, minos internal document 11466," April 2016.

[129] A. Aurisano, "A position paper on constructing systematic covariance matrices for the 2014 sterile analysis, minos document 10581," April 2014. 
[130] S. Dytman, H. Gallagher, and M. Kordosky, "Hadronic Shower Energy Scale Uncertainty in the MINOS Experiment," 2008.

[131] J. Evans, "Absolute (calorimetric) shower energy uncertainty, minos document 7173," April 2010.

[132] J. Evans, K. Lang, and R. Nichol, "Position paper on the 2010 analysis of charged current events from runs i, ii and iii, minos document 7218," April 2010.

[133] P. Adamson et al., "Search for sterile neutrino mixing in the MINOS long baseline experiment," Phys.Rev., vol. D81, p. 052004, 2010.

[134] A. Radovic and M. Kordosky, "A position paper describing a hadron production uncertainty covariance matrix, minos document 10569," April 2014.

[135] M. Bonesini, A. Marchionni, F. Pietropaolo, and T. Tabarelli de Fatis, "On Particle production for high-energy neutrino beams," Eur. Phys. J., vol. C20, pp. 13-27, 2001.

[136] P. Adamson et al., "A Study of Muon Neutrino Disappearance Using the Fermilab Main Injector Neutrino Beam," Phys.Rev., vol. D77, p. 072002, 2008.

[137] A. Timmons, "Position paper: Beam optic systematic matrices for the 2014 sterile analysis, minos document 10580," April 2014. 
[138] A. Timmons, "Minos and minos+ beam focusing covariance matrices, minos document 10964," March 2015.

[139] G. J. Feldman and R. D. Cousins, "A Unified approach to the classical statistical analysis of small signals," Phys.Rev., vol. D57, pp. 3873-3889, 1998.

[140] J. Todd, "Flux prediction and errors in the fhc sterile analysis á la ppfx, minos internal document 12905," March 2018.

[141] A. V. Lebedev, Ratio of pion kaon production in proton carbon interactions. PhD thesis, Harvard U., 2007.

[142] A. Aurisano, R. Chen, J. Evans, J. Todd, and L. Whitehead, "Sterile neutrino systematic correlations, minos internal document 11710," December 2016.

[143] J. Todd, "Fhc sterile analysis updates, minos internal document 11721," January 2017.

[144] G. Golub and C. Van Loan, Matrix Computations. Johns Hopkins University Press, 3 ed., 1996.

[145] J. Todd, "Position on the minos \& minos + combined fhe sterile neutrino analysis, minos internal document 11753," February 2017.

[146] P. Adamson et al., "Search for sterile neutrinos in minos and minos+ using a two-detector fit: Supplemental materials, minos internal document 13055," May 2018. 
[147] J. Todd, "Fixed atmospheric oscillations in the sterile fit, minos internal document 12510," October 2018. 


\section{Vita}

Simon De Rijck was born in Aalst, Belgium, the son of Johan De Rijck and Hilde De Turck. He received Bachelor of Science and Master of Science degrees in Physics and Astronomy from Ghent University (Belgium) in 2011 and 2013, respectively. He started the Physics Ph.D. program at the University of Texas at Austin in August, 2013.

Email address: simonderijck@gmail.com

This dissertation was typeset with $\mathrm{AT}_{\mathrm{E}} \mathrm{X}^{\dagger}$ by the author.

\footnotetext{
${ }^{\dagger} \mathrm{LT}_{\mathrm{E}} \mathrm{X}$ is a document preparation system developed by Leslie Lamport as a special version of Donald Knuth's $\mathrm{T}_{\mathrm{E}} \mathrm{X}$ Program.
} 